\title{
Implementation of structural bioinformatics in thromboinflammation studies
}

Citation for published version (APA):

Liu, X. (2021). Implementation of structural bioinformatics in thromboinflammation studies. [Doctoral Thesis, Maastricht University]. Ridderprint. https://doi.org/10.26481/dis.20211209xl

Document status and date:

Published: 01/01/2021

DOI:

10.26481/dis.20211209xl

Document Version:

Publisher's PDF, also known as Version of record

\section{Please check the document version of this publication:}

- A submitted manuscript is the version of the article upon submission and before peer-review. There can be important differences between the submitted version and the official published version of record.

People interested in the research are advised to contact the author for the final version of the publication, or visit the DOI to the publisher's website.

- The final author version and the galley proof are versions of the publication after peer review.

- The final published version features the final layout of the paper including the volume, issue and page numbers.

Link to publication

\footnotetext{
General rights rights.

- You may freely distribute the URL identifying the publication in the public portal. please follow below link for the End User Agreement:

www.umlib.nl/taverne-license

Take down policy

If you believe that this document breaches copyright please contact us at:

repository@maastrichtuniversity.nl

providing details and we will investigate your claim.
}

Copyright and moral rights for the publications made accessible in the public portal are retained by the authors and/or other copyright owners and it is a condition of accessing publications that users recognise and abide by the legal requirements associated with these

- Users may download and print one copy of any publication from the public portal for the purpose of private study or research.

- You may not further distribute the material or use it for any profit-making activity or commercial gain

If the publication is distributed under the terms of Article $25 \mathrm{fa}$ of the Dutch Copyright Act, indicated by the "Taverne" license above, 


\section{Implementation of structural bioinformatics in thromboinflammation studies}

Xiaosong Liu 
ISBN: 978-94-6416-911-9

Cover: Nino Bolink (Ridderprint) and Xiaosong Liu Layout: Xiaosong Liu

Printed by: Ridderprint BV, www.ridderprint.nl

Thesis Universiteit Maastricht

Copyright $\odot 2021$ by Xiaosong Liu 


\title{
Implementation of structural bioinformatics in thromboinflammation studies
}

\author{
DISSERTATION \\ To obtain the degree of Doctor at the Maastricht University, \\ on the authority of the Rector Magnificus, \\ Prof. Dr. Rianne M. Letschert \\ in accordance with the decision of the Board of Deans, \\ to be defended in public \\ on Thursday $9^{\text {th }}$ of December, 2021, at 12:00 hours \\ by
}

Xiaosong Liu 
Supervisor(s):

Dr. G.A.F. Nicolaes

Prof. Dr. C.P.M. Reutelingsperger

Co-supervisor:

Dr. K. Wichapong

\section{Assessment Committee:}

Prof. Dr. C.G. Schalkwijk (Chairman)

Dr. I. Dijkgraaf

Dr. P. Garcia de Frutos (University of Barcelona, Spain)

Prof. Dr. G.R.M.M. Haenen

Prof. Dr. W. Vranken (Free University of Brussels, Belgium)

Financial support by China scholarship Council (Grant No.201606350089) is gratefully acknowledged. 


\section{Table of contents}

Chapter 1 General introduction

Chapter 2 Rational modulator design by exploitation of protein-protein complex structures

Chapter 3 PAD4 takes charge during neutrophil activation:

impact of PAD4 mediated NET formation on immune-mediated disease

Chapter 4 Autocitrullination of PAD4 does not alter its enzymatic activity: in vitro and in silico studies

Chapter 5 Pseudonaja textilis venom-derived factor Va retains structural integrity following proteolysis by activated protein $\mathrm{C}$

Chapter 6 Rational design of peptide agonists activating C-X-C

chemokine receptor type 4 (CXCR4)

Chapter 7 General discussion

Appendices

appendix I: English summary

appendix II: Nederlandse samenvatting

appendix III: Chinese summary

appendix IV: Impact paragraph

appendix V: Curriculum vitae

appendix VI: List of publications 

Chapter

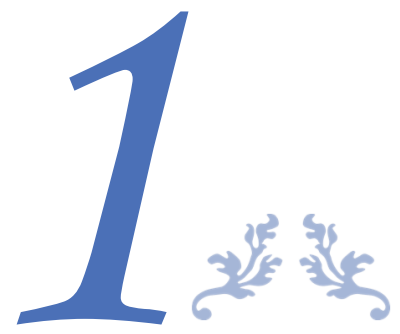

General introduction 



\subsection{Introduction}

The drug discovery process comes with large investments in terms of resources and time since it can cost more than 1.3 billion US dollars and takes from 10 to 20 years to develop a new drug and bring it to the market ${ }^{1-3}$. Structural bioinformatics is an effective and powerful approach that is widely used to identify and optimize bioactive compounds in the development of novel bioactive molecules that may evolve into drugs (Figure 1). By applying various computational tools and methods in different stages of drug discovery, structural bioinformatics can considerably reduce both costs and duration of the drug discovery process ${ }^{4}$. Frequently utilized tools and techniques include homology modeling, virtual screening (VS), molecular dynamic (MD) simulations, binding free energy calculation and structureactivity relationships (SARs). This thesis introduces the rational design of peptidic modulators through a combination of various structural bioinformatics methods (chapter 2 ) and shows its applications to the design and optimization of bioactive molecules for a specific target (chapter 6). Moreover, this thesis demonstrates that some structural bioinformatics tools (e.g., molecular docking, molecular dynamic simulations and binding free energy calculation) can assist researchers in solving problems related to protein structure and function that are difficult to elucidate by experimental methods (chapter 4 and chapter 5). In this general introductory chapter, different structural bioinformatics tools used in this thesis are described, as well as several cardiovascular therapeutic targets.
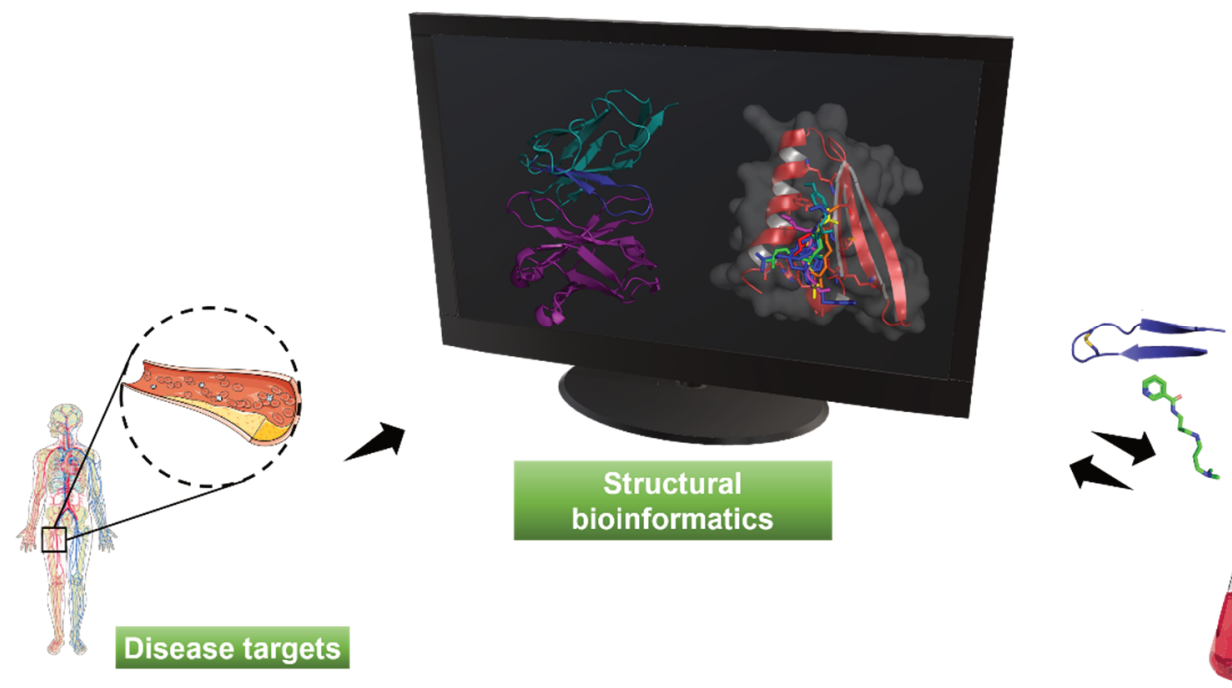

In vitro

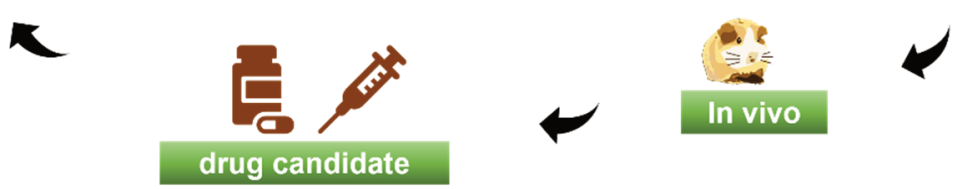

Figure 1. The role of structural bioinformatics in drug discovery. The role of structural bioinformatics in drug discovery. Structural bioinformatics studies the knowledge of $3 D$ structure of diseases target and delivers information which guide experiments moving forward. The information contains protein structure stability, protein structure and function relationships, screened molecules which satisfy various parameters, rational designed molecules and more. 


\subsection{Computational methods in drug discovery and design}

\subsubsection{Homology modeling}

There is a huge and expanding gap between available sequences and experimentally solved protein 3D protein structures ${ }^{5}$. Therefore, homology modeling provides a useful alternative to obtain protein 3D structures by means of 3D structure prediction. Homology modeling can create reliable structure models, that may serve as druggable target structures, based on the availability of a target sequence and suitable template structures ${ }^{6}$. Homology modeling consists of multiple steps (as summarized in Figure 2) to generate a model structure: 1) identify and select a 3D template; 2) align the target sequence to the template sequence; 3 ) build the model based on the alignment; and 4) optimize and validate the model.

1) Identify and select the template

Unknown structure

Known structure

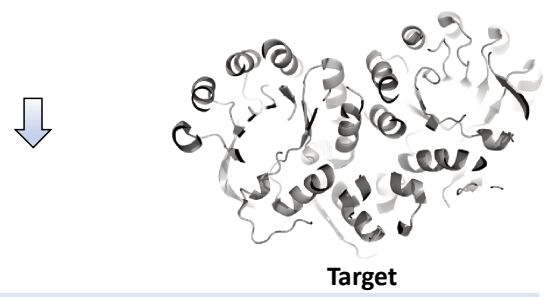

2) Align target sequence to template sequence

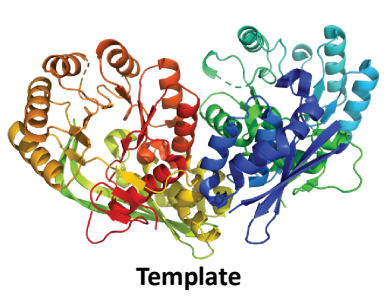

Alignment

$\preccurlyeq$\begin{tabular}{l|l}
\hline Target seqeunce & - - TVQWHFCSVGTQNEILTIHFT - - \\
\hline Template seqeunce & - -VVQWHLTS - - -VDEIVPVHLS- - \\
\hline
\end{tabular}

3) Build the model based on the alignment

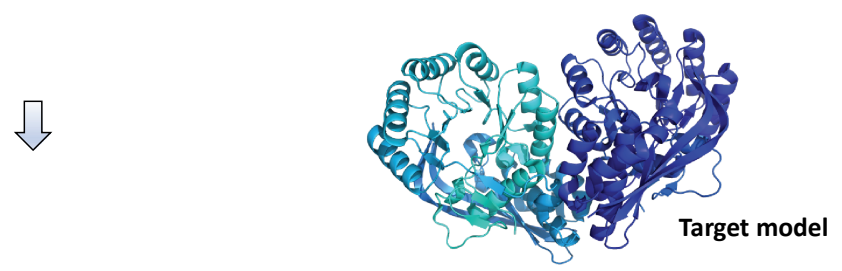

4) Optimize and validate the model.

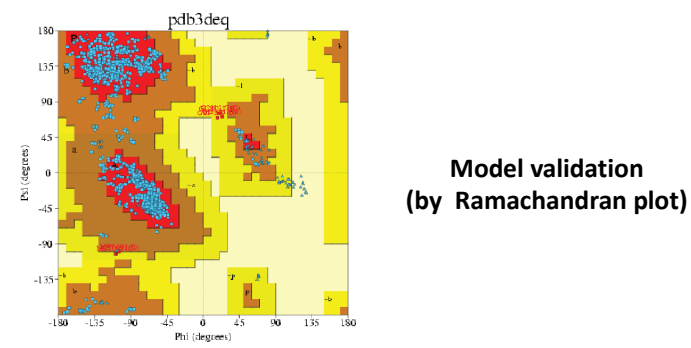

Figure 2. Schematic representation of homology modeling. Homology model consists of four steps to produce an accurate model structure: 1) identify and select the template structure according to target sequence; 2) align target sequence to template; 3) built the target model based on template sequence; 4) model validation via various methods (Ramachandran plot is an example of validation method) 
When selecting a homologue of known 3D structure as the template, the sequence identity (between the target and the template) associated with sequence length influences the model quality ${ }^{7,8}$ (Figure 3). In practice, the target sequence is sent to the BLAST server ${ }^{9}$ to search for possible templates with known structures in Protein Data Bank (PDB). For a target of 100 residues long, for example, a sequence identity of $>30 \%$ is sufficient to result in a reliable homology model, as indicated by the "safe zone" (Figure 3). If the sequence identity between the target sequence and template sequence would be $<30 \%$, their homologous relationship becomes unreliable. In this situation, alternative approaches such as template consensus sequences ${ }^{10}$ (based on the multiple sequence alignments) and 3D profile analysis $^{11}$ (based on the secondary structures likeness) can be employed to build a reliable model.

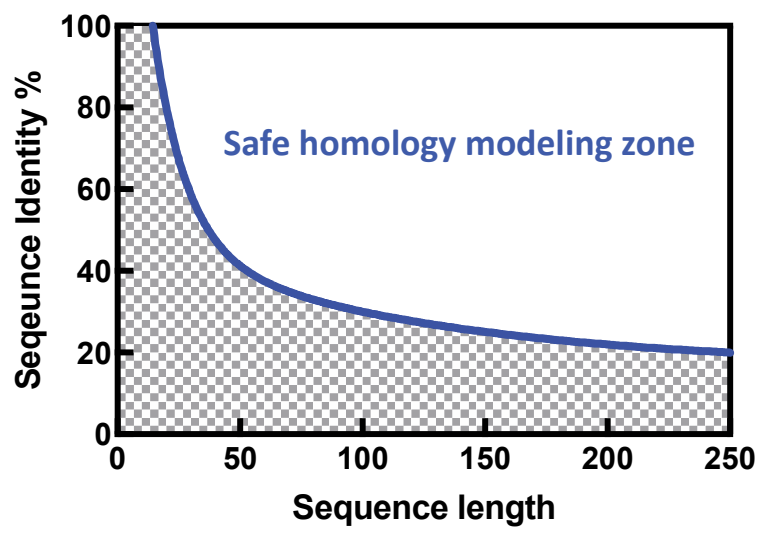

Figure 3. Zones in homology modeling. It shows how much identity is needed for a certain length of target and provides the guidance for homology modeling to yield reliable results. As such a "safe" and an "twilight" zone are defined.

After selection of the template, the model building process can be achieved by applying modifications to the template structure, such as mutations, deletions or insertions, based on the alignment. Energy optimizations are used to relax side chains of modified residues and remove clashes between atoms. However, problems can arise from the structure prediction of loops that are missing in the template structure, as they often correspond to unaligned regions, more conformational flexible regions, or regions with low electron density, and are difficult to solve. There are two strategies that can be applied for loop modeling, with or without using a template. When using a template, a PDB database with all known structures is searched for the conformation of this segment. When no template is used, loop conformations are generated by a manner of randomly sampling ( $a b$ initio method) combined with energy minimization, with the best loop conformation, the one with the lowest energy, being selected. After all these operations, the draft model will be energy minimized to relax the whole model.

It is important to include a validation step before further application, in order to detect any errors in the constructed model. This is typically done using Ramachandran plot which analyzes the basic stereochemistry of the model (parameters including bond length, torsion angle, and rotational angle), residue geometry, and the resemblance between model and template. This step does not need any protein-specific knowledge. Examples of programs that are commonly used for structure model validation and homology modeling are listed in Table 1. 
Table 1. Commonly used software in homology modeling.

\begin{tabular}{|c|c|c|c|}
\hline Method & Software/Source & Ref & Annotation \\
\hline $\begin{array}{l}\text { Homology } \\
\text { modeling }\end{array}$ & $\begin{array}{l}\text { YASARA Homology } \\
\text { Modeling } \\
\text { MODELLE } \\
\text { SWISS-MODEL } \\
\text { I-TASSER }\end{array}$ & $\begin{array}{l}12 \\
13 \\
14 \\
15\end{array}$ & $\begin{array}{l}\text { Knowledge-based } \\
\text { Ab initio } \\
\text { Knowledge-based } \\
\text { Knowledge-based }\end{array}$ \\
\hline $\begin{array}{c}\text { Protein structure } \\
\text { validation and } \\
\text { quality }\end{array}$ & $\begin{array}{l}\text { Procheck } \\
\text { Prosa-web } \\
\text { MolProbity } \\
\text { NQ-Flipper }\end{array}$ & $\begin{array}{l}17 \\
18 \\
19 \\
20\end{array}$ & $\begin{array}{c}\text { Extensive structural check. Also checks } \\
\text { ligands, and features which cannot be } \\
\text { extracted from known proteins, such as } \\
\text { electrostatic and van der Waals interactions; } \\
\text { Checks main-chain/side-chain parameters, } \\
\text { stereochemical properties by residue; } \\
\text { Calculates knowledge-based energy score; } \\
\text { Calculates all atom contacts, including } \\
\text { hydrogen bonds and van der Waals at } \\
\text { interacting interfaces; } \\
\text { Diagnoses problematic Asn and Gln rotamers } \\
\text { from crystal structures }\end{array}$ \\
\hline
\end{tabular}

\subsubsection{Structure-Based Virtual Screening (SBVS)}

Virtual screening (VS) is a structural bioinformatics tool that has been successfully applied to discover lead compounds with biological activity towards their therapeutic target. The idea behind VS is that a large compound library (millions of compounds) will go through a set of computational filters to be reduced to a small compound library (hundreds to thousands of compounds) for experimental testing. This method aims to deliver new drug candidates in a more efficient way than traditional library screens. VS can be classified as either structure-based virtual screening (SBVS) ${ }^{21}$ or ligand-based virtual screening (LBVS). The SBVS requires the knowledge of atomic-level structural information of the protein target, whereas the LBVS attempts to find similar compounds to known bioactive ligands. As the SBVS is mainly applied in this thesis, we focus on the introduction to SBVS which is established using molecular docking as the basis. The workflow (Figure 4) of the SBVS method includes 1) target preparation; 2) ligand preparation; 3) molecular docking; and 4) post-docking analysis.

\section{1) Target preparation}

3D structures of target proteins can be retrieved from the Protein Data Bank which contains all publicly accessible 3D protein structures experimentally determined by X-ray crystallography, NMR, or cryo-EM. In addition, the PDB-REDO 22 databank provides optimized and refined entries of the PDB with electron density maps, a description of model changes, and a wealth of model validation data. The PDBe-KB ${ }^{23}$ (PDB in Europe - Knowledge Base) server displays the PDB archive with additional biological information including structural and functional annotations. For targets without experimentally determined 3D structure, the complete structural model or the 3D model of the part of the target that is of interest, such as a protein domain, can reliably and accurately be predicted through homology modeling based on the target sequence and a homologous protein of known structure (see section, Homology modeling). 

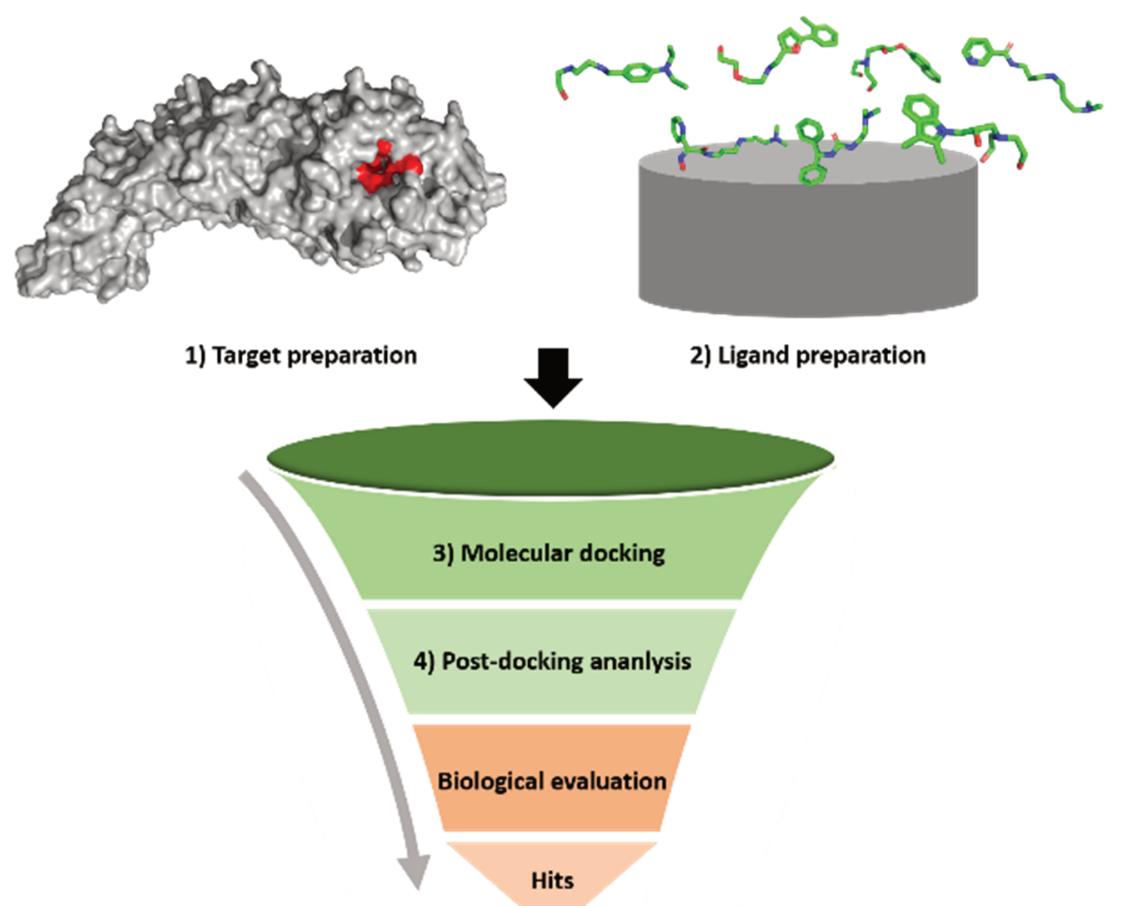

Figure 4. The flowchart of Structure-Based Virtual Screening. Biological evaluation indicates functional or binding test in vitro.

One needs to find one or more ligand binding sites (binding pockets) in the prepared target structure before being molecular docking ${ }^{24}$. By in-depth analysis of experimentally measured protein-ligand interactions collected from several databases, such as ChEMBL ${ }^{25}$, PubChem ${ }^{26}$, and DrugBank ${ }^{27}$, researchers are able to precisely identify and characterize a ligand binding region. However, the majority of proteins have lacking structural and functional annotations. Therefore, binding site prediction represents a useful strategy widely applied in SBVS ${ }^{28}$. Table 2 lists commonly used software or algorithms for pocket prediction, which can assist to find binding pockets in unannotated proteins based on different mechanisms, such as inspection of homologous templates (template-based), relying on the local chemical properties of 3D structure surfaces (machine learning), or identifying the favorable binding regions of known ligands (ligand-based).

Table 2. Commonly used software in target preparation.

\begin{tabular}{cccc}
\hline Method & Software/Source & Ref & Annotation \\
\hline \multirow{3}{*}{ Target structure extraction } & Protein Data Bank & 29 & Crystallographic structures \\
& PDB_redo & 22 & Validated structures \\
& PDBe-KB & 23 & Annotated structures \\
& Fpocket & 30 & Comprehensive \\
& SiteHound-web & 31 & Ligand-based \\
Binding pocket prediction & eFindSite & 32 & Template-based \\
& bSiteFinder & 33 & Template-based \\
& P2Rank & 34 & Machine learning \\
\hline
\end{tabular}




\section{2) Ligand preparation}

Examples of well-established ligand databases (DBs) are listed in Table 3. A common practice in our laboratory is to download a large compound library and subject this to the FAF-Drugs 4 online server ${ }^{35}$ that passes these compounds through several filters. The rationale behind this step is to prepare a compound library of proper quality. Compounds that may serve as potential starting points for further optimization are preserved in this step. The filters used can filter out molecules with undesirable moieties and substructures involved in terms of toxicity, PAINS (Pan Assay Interference Compounds), and molecules that do not meet desired drug's physico-chemical properties. Compounds with PAINS moieties are promiscuous since they are frequently reported as "false" actives by many different assays towards different targets ${ }^{36}$. The physico-chemical properties of a compound are important for the assessment of druglikeness, including ADME properties (absorption, distribution, metabolism, excretion $)^{37}$. A commonly used method for druglikeness evaluation is Lipinski's rule of five (RO5) ${ }^{38}$ : molecular weight $(\mathrm{MW})<500 \mathrm{Da}, \log \mathrm{P}(\mathrm{P}=$ octanol/water partition coefficient $)<5$, hydrogen bond donors $<5$, hydrogen bond acceptors $<10$.

Table 3. Commonly used compound databases and softwares in ligand preparation.

\begin{tabular}{|c|c|c|c|}
\hline Method & Software/Source & Ref & Annotation \\
\hline \multirow{5}{*}{$\begin{array}{l}\text { Public compound } \\
\text { databases }\end{array}$} & $\overline{Z I N C}$ & 39 & over 230 million \\
\hline & PubChem & 26 & over 100 million \\
\hline & Binding DB & 40 & 1.9 million \\
\hline & DrugBank & 27 & 13,603 drug entries \\
\hline & ChEMBL & 25 & Over 1 million \\
\hline $\begin{array}{l}\text { Commercial } \\
\text { compound } \\
\text { databases }\end{array}$ & $\begin{array}{l}\text { Chembridge } \\
\text { Life Chemicals } \\
\text { Maybridge }\end{array}$ & $\begin{array}{l}\text { https://chembridge.com } \\
\text { https://lifechemicals.com } \\
\text { http://maybridge.com }\end{array}$ & $\begin{array}{c}1.3 \text { million in stock } \\
\text { Over } 490,000 \text { in } \\
\text { stock } \\
\text { over } 53,000 \text { in } \\
\text { stock }\end{array}$ \\
\hline \multirow{3}{*}{ Filter } & FAFDrugs4 & 35 & \\
\hline & FILTER (openeye) & 41 & Standalone \\
\hline & $\begin{array}{c}\text { Open Babel (-filter } \\
\text { option) }\end{array}$ & 42 & Standalone \\
\hline \multirow{4}{*}{$\begin{array}{l}\text { Ligand 3D structure } \\
\text { generation }\end{array}$} & Open Babel & 42 & Standalone \\
\hline & OMEGA (OpenEye) & 41 & Standalone \\
\hline & ConfGen & 43 & Standalone \\
\hline & RDKit & 44 & Standalone \\
\hline
\end{tabular}

Compounds derived from DBs are commonly in a two-dimensional (2D) format, and conversion into a three-dimensional (3D) conformation is needed before subjecting them to molecular docking. 3D conformations of compounds are generated by sampling rotatable bonds and determining torsion angles. A low-energy conformation of a compound represents a thermodynamically favorable conformer. The protonation state, charges as well as tautomeric states and stereochemistry of compounds also need to be properly defined and assigned, since these parameters can affect the docking accuracy. Most of the docking programs contain modules to prepare compounds so as to assist the user to prepare ligands before a docking run. 


\section{3) Molecular docking}

Molecular docking is a method used to predict how a ligand binds to a receptor and gives a docking score to distinguish good from bad binding modes ${ }^{45}$. In general, there are two steps in the docking process (Figure 5).

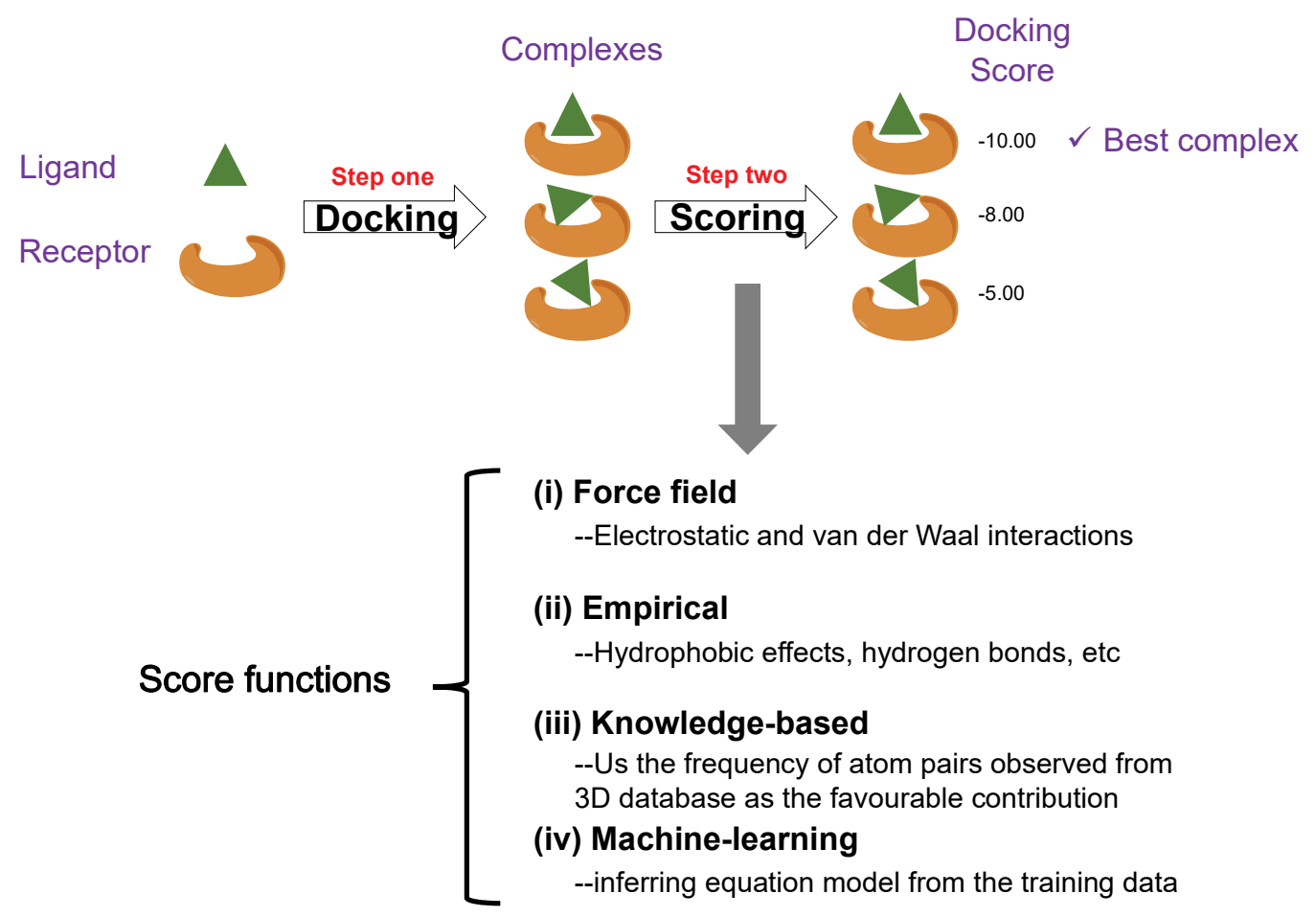

Figure 5. The two-steps of molecular docking process. Step one is the process of docking ligands onto their receptor, after which different complexes are produced, step two comprises the calculation of a score to the ligand:receptor complex based on a score function that incorporates all essential types of interactions to evaluate docking pose and binding potency.

Firstly, the docking process allows ligand conformations/orientations sampling on the specific targeted region to generate the best binding poses. Commonly utilized docking tools are listed in Table 4, which can be identified as rigid ligand docking (treats both ligand and receptor as rigid entities) and flexible ligand docking (treats receptor as a rigid body and treats ligand as fully flexible). Second, the scoring function is used to evaluate compound docking poses and predict their binding potencies to the receptor. There are four classifications of scoring functions46: (i) force-field; (ii) empirical; (iii) knowledge-based and (iv) machine-learning. The force-field score functions evaluate the binding affinity by the sum of energy terms (e.g. van der Waal and electrostatic interactions) between atoms of two molecules in the complex ${ }^{46}$. The empirical scoring functions count the various atomic interactions based on the contact surface between the protein and ligand, such as hydrophobic effects and hydrogen bonds using an established model ${ }^{47}$. The knowledge-based score functions analyze the frequency of different types of atom contacts based on all available 3D structures and use them as the favorable contribution to the binding affinity estimation ${ }^{48}$. The machine learning behaves differently from the first three scoring functions. Machine learning infers scoring from an equation model of training data in an unbiased manner rather than using a pre-established one ${ }^{49}$. 
Table 4. Commonly used software in molecular docking.

\begin{tabular}{cccc}
\hline Method & Software/Source & Ref & Annotation \\
\hline & FRED (OpenEye) & 50 & Rigid ligand docking \\
Surflex (SYBYLX) & 51 & Flexible ligand docking \\
& Glide (Schrödinger) & 52 & Flexible ligand docking \\
Docking & Dock 6 & 53 & Rigid or flexible ligand docking \\
& AutoDock Vina & 54 & Flexible ligand docking \\
& ICM-dock (ICM-Pro) & 55 & Flexible ligand docking \\
& GOLD & 56 & Flexible ligand docking \\
\hline
\end{tabular}

\section{4) Post-processing of molecular docking}

Although molecular docking has been successfully used in virtual screening, there are limitations with the docking output, such as high rates of "false positives"57-59 and the relative disregard of receptor flexibility in several standard docking procedures ${ }^{60,61}$. To overcome these limitations and improve docking outcomes, several efficient post-docking processes can be applied (e.g. consensus scoring, compound diversity, visual inspection, ensemble docking ${ }^{62}$, refining docked poses by Molecular Mechanics Generalized Born or Poisson-Boltzmann surface area (MM/GB(PB)SA)63, $\left.{ }^{64}\right)$. The consensus scoring method combines different docking scoring functions and selects compounds that have good ranks in all score functions, which yields higher enrichment of true positives in virtual screening ${ }^{65-67}$. Ensemble docking docks ligands on a set of receptor conformations rather than on a single conformation, in an approximation to consider receptor flexibility, especially for targets that have a flexible binding site or ligand induced conformational changes in the binding site ${ }^{62}$. Multiple protein conformations can be obtained from different experimentally solved 3D-crystal structures. Likewise, multiple protein conformers can be snapshots extracted from molecular dynamic (MD) simulations ${ }^{68-70}$. The diversity of docking score prioritized compounds can be improved by including more compounds from clusters based on compounds $2 \mathrm{D}$ similarities ${ }^{71}$. Alternatively, the MM/GB(PB)SA method combined with short MD simulations for binding free energy calculation (see section, Binding free energy (BFE) calculation) can be used to refine docking poses and provide a prediction of relative ligand binding affinities to receptor ${ }^{72,73}$. This method has successfully yielded binding affinities that correlated with experimental results $^{74}$ and generated improved enrichment with the recovery of actives from decoys ${ }^{75-77}$.

\subsubsection{Molecular dynamic (MD) simulations}

The protein structure derived from experimental methods only provides generally limited information on dynamic protein properties. Therefore, molecular dynamic (MD) simulation was introduced as a powerful computational tool to study motions of biological macromolecules (such as protein, membrane, nucleic acids) based on Newton's laws of motion ${ }^{78}$. Briefly, given the positions of all atoms of a biomolecule, the three-dimensional position of each atom at every point of simulated time can be saved in trajectories by calculating forces on them ${ }^{78}$. Therefore, MD simulation is able to provide details on the conformational changes and fluctuations of biomolecules over simulation time. For example, the stability of protein structure can be monitored by calculation of the root-mean-square deviation (RMSD) of atomic 
positions as a function of simulation time; the protein flexibility can be inspected by per-residue rootmean-square fluctuation (RMSF) over MD trajectories (Figure 6). Through MD, researchers gain better understanding of protein stability ${ }^{79,80}$ and ligand binding ${ }^{81,82}$. Besides, MD simulations are able to provide an insight into the protein or peptide folding process ${ }^{83,84}$, or into the interactions between proteins in complexes ${ }^{85,86}$. Moreover, ensemble docking uses structural conformations of the protein which can be obtained from MD trajectories in structure based virtual screening ${ }^{68-70}$. Binding affinity prediction between molecules that form a complex starts with an MD simulation of the complex 87,88 . With the improved computational calculation power and more accurate force fields available, the role of $M D$ simulation in drug discovery is likely to increase ${ }^{89}$. There are many popular simulation MD programs, such as CHARMM $^{90}$, AMBER ${ }^{91}$ and GROMACS ${ }^{92}$. AMBER is the main tool used in this thesis for MD simulation.
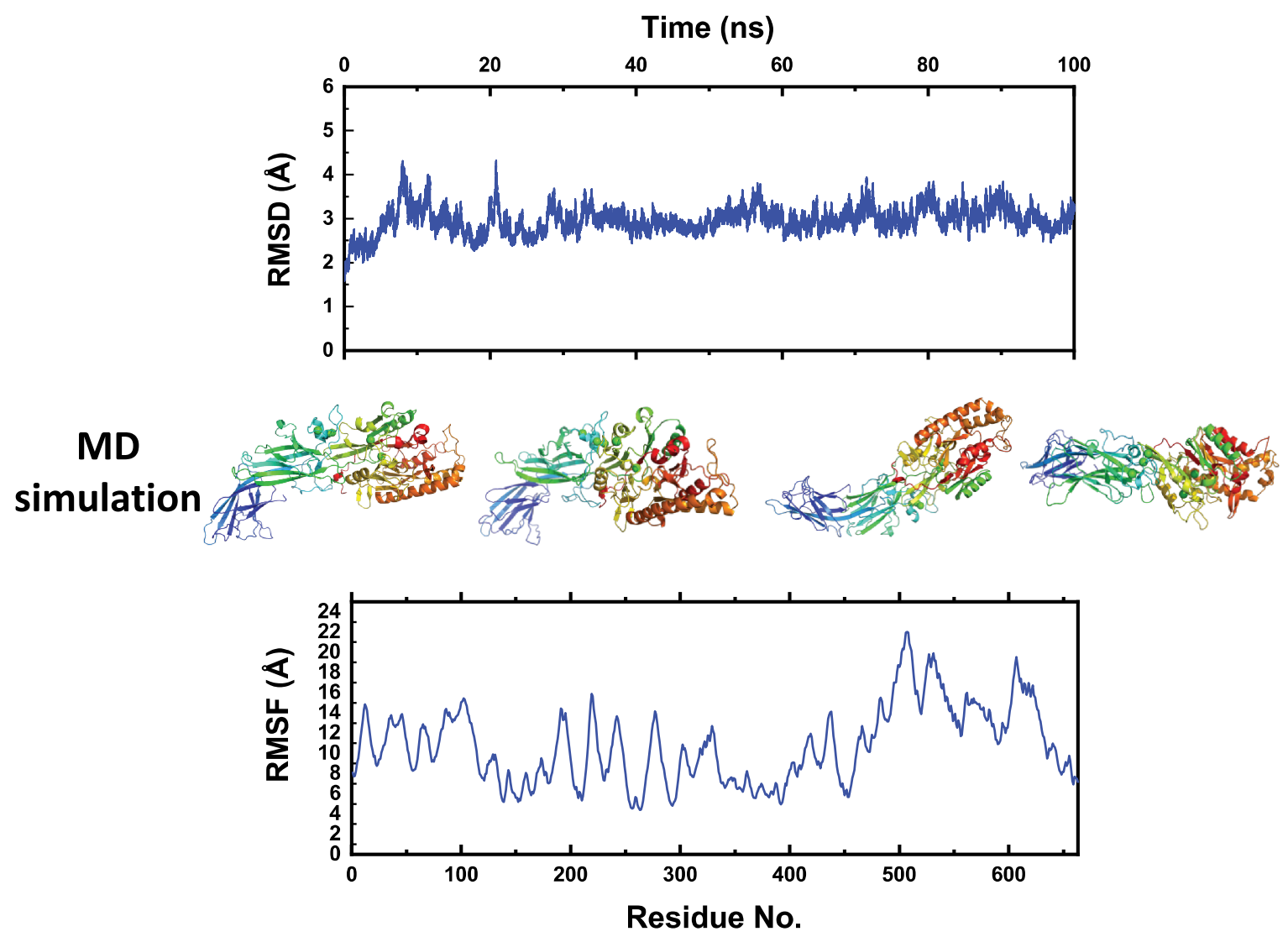

Figure 6. The RMSD and RMSF analysis of protein based on the MD simulation. The RMSD indicates protein stability, the per-residue root-mean-square fluctuation (RMSF) of protein calculated over MD trajectories indicates the structure flexibility.

\subsubsection{Binding free energy (BFE) calculation}

There are several computational methods to calculate the binding free energy (BFE) between receptor and ligand, such as free energy perturbation (FEP $)^{93}$, thermodynamic integration $(\mathrm{TI})^{94}$, linear interaction energy (LIE) ${ }^{95}$, molecular mechanics Generalized Born or Poisson-Boltzmann MM/GB(PB)SA ${ }^{96}$. The $\mathrm{MM} / \mathrm{GB}(\mathrm{PB}) \mathrm{SA}$ method is the most popular and widely used method for BFE calculation in computer 
aided drug design ${ }^{72,96,97}$. It has advantages in terms of computational cost while yielding an acceptable accuracy, and it can be applied in various systems (such as protein-protein, protein-ligand, and proteinRNA/DNA systems). The MM/GB(PB)SA based BFE calculation usually begins with MD simulations of the complex (receptor and ligand). Ensembles of structures obtained by extracting snapshots from equilibrated MD trajectories can be used for the BFE calculation. As the MM/GBSA or MM/PBSA method was employed for BFE calculations in this thesis, its detailed calculation is described by the equations below:

$$
\Delta G_{\text {binding }}=G_{\text {complex }}-\left(G_{\text {receptor }}+G_{\text {ligand }}\right)
$$

Where the total binding free energy $\left(\Delta G_{\text {binding }}\right)$ represents the free energy differences between bound state $\left(G_{\text {complex }}\right)$ and free state $\left(G_{\text {receptor }}+G_{\text {ligand }}\right)$ of two solvated molecules (receptor and ligand). The $\Delta G_{\text {binding }}$ can be further described by equations (2)-(4):

$$
\Delta G_{\text {binding }}=\Delta H-T \Delta \mathrm{S}=\Delta E_{M M}+\Delta G_{\text {sol }}-T \Delta \mathrm{S}
$$

in which

$$
\begin{aligned}
& \Delta E_{M M}=\Delta E_{\text {int }}+\Delta E_{\text {ele }}+\Delta E_{v d W} \\
& \Delta G_{\text {sol }}=\Delta G_{\text {polar }}+\Delta G_{\text {nonpolar }}
\end{aligned}
$$

Where $\Delta H$ and $-T \Delta S$ are the enthalpy and entropy change upon ligand binding, respectively. The enthalpy calculation is composed of molecular mechanical energy change $\Delta E_{M M}$ in gas-phase and the solvent free energy change $\Delta G_{\text {sol }} . \Delta E_{M M}$ is the sum of intra-molecular energy change $\Delta E_{\text {int }}$ (bond, angle and dihedral energies), electrostatic energy change $\Delta E_{\text {ele }}$, van der Waals energy change $\Delta E_{v d W}$, calculated using molecular mechanics (MM). $\Delta G_{\text {sol }}$ is the sum of polar contribution $\Delta G_{\text {polar }}$ and nonpolar contribution $\Delta G_{\text {nonpolar }}$ between solute and solvent. The polar solvent free energy is calculated by using either GB (Generalized Born) or PB (Poisson Boltzman) model, while the nonpolar part is usually estimated by the solvent accessible surface area (SASA) of solute ${ }^{98}$. The entropy calculation is computationally expensive and generally produces less accurate results ${ }^{99}, 100$. In cases where relative binding affinities in a same system are aimed at, the entropy term can be neglected (such as when similar-sized ligands binding to the same receptor are compared).

\subsection{Therapeutic targets in cardiovascular diseases}

\subsubsection{Protein Arginine Deiminase 4 (PAD4)}

PAD4 is a member of the protein arginine deiminases (PADs) family which modifies arginine residues (positively charged) into citrulline residues (neutrally charged) ${ }^{101}$. PAD4 is mainly expressed in granulocytes and PAD4-mediated histone citrullination is essential for the formation of neutrophil extracellular traps (NETs) ${ }^{102-104}$. NETs are described as an innate immune response to kill invasive pathogens; however, dysregulated NETs promote thrombosis by providing a scaffold for platelet aggregation $^{105,106}$ (Figure 7). In deep vein thrombosis, PAD4 knockout mice have significantly reduced thrombus formation compared to wild-type mice, however, they can be rescued from lethal thrombosis 
by intravenous administration of wild-type neutrophils containing PAD4 ${ }^{105}$. During NETosis (NET formation), PAD4 mediated chromatin decondensation leads to the release of intracellular compounds such as histones into the extracellular space. Extracellular histones are toxic to cells and contribute to the pathogenesis of fulminant inflammatory diseases such as sepsis ${ }^{107,108}$. In addition, activated neutrophils release PAD4 which can citrullinate plasma proteins and thereby contribute to autoimmunity 109,110 and a prothrombotic state ${ }^{111,112}$. Taken together, PAD4 represents a potential therapeutic target in NETs-related cardiovascular diseases and autoimmunity.

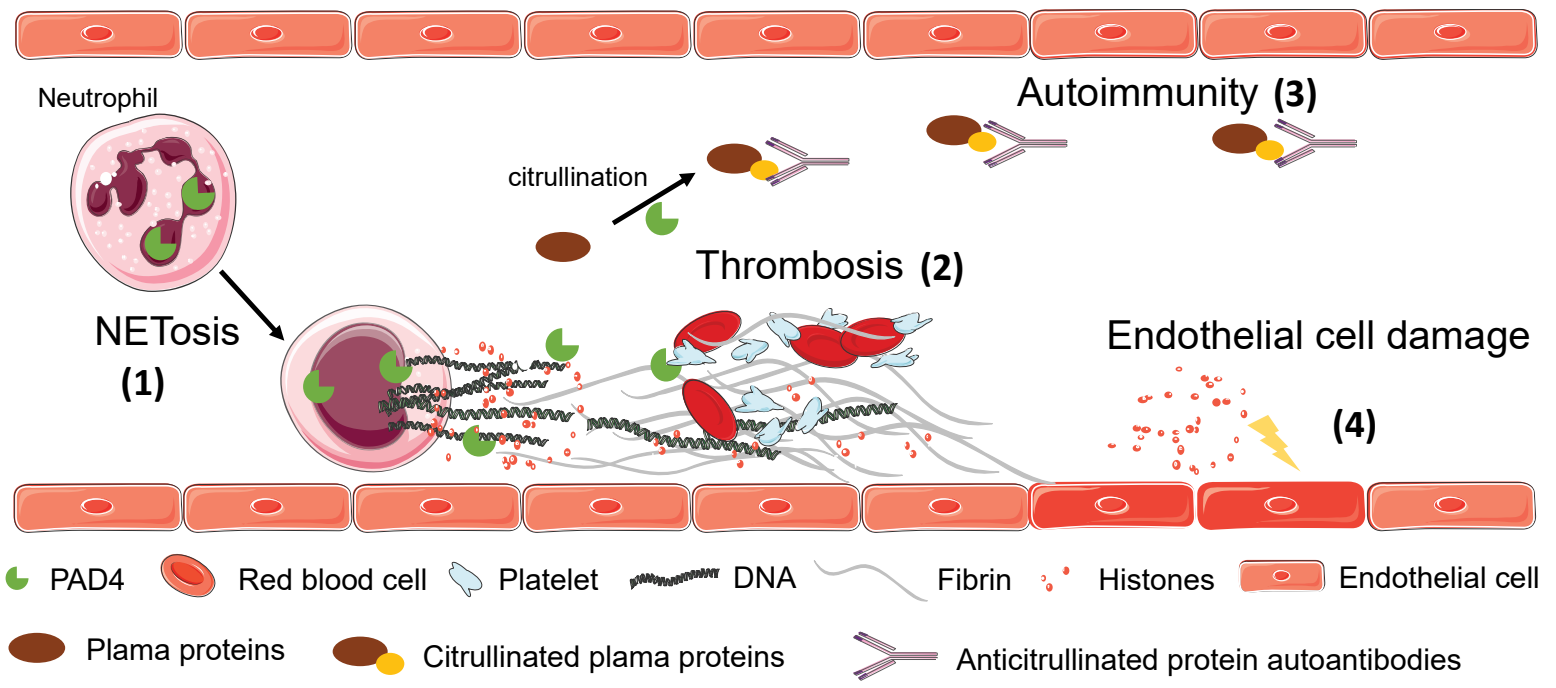

Figure 7. PAD4 involvement in cardiovascular diseases. PAD4 drives NETosis through citrullination of nuclear histones (1); NET structure and NET components serve as a scaffold for thrombus formation (2); NET released PAD4 can citrullinate plasma proteins and therefore cause autoimmunity (3); Extracellular histones are toxic to cells and cause cell and tissue damage (4).

\subsubsection{Coagulation Factor V (FV)}

Coagulation Factor $\mathrm{V}(\mathrm{FV})$ plays an essential role in the regulation of both procoagulant and anticoagulant pathways. FV consists of six domains (A1-A2-B-A3-C1-C2) (Figure 8), and serves as an anticoagulant cofactor of activated protein C (APC) for the inactivation of factor VIIla and factor $\mathrm{Va}^{113}$, 114. FV is activated by removal of the B domain through proteolytic cleavage after the residues Arg709, Arg1018, and Arg1545 by Factor Xa, meizothrombin or thrombin ${ }^{115-117}$. Activated FV (FVa) is composed of a heavy chain (A1-A2) and a light chain (A3-C1-C2) which non-covalently associate with each other (Figure 8). FVa is procoagulant ${ }^{118}$ and contributes to a hypercoagulable state if not inactivated timely as is illustrated by the mutation of Factor V Leiden which results in impaired inactivation by APC 119-121. The activity of FVa is downregulated by APC cleavage at positions of $\mathrm{Arg}^{306}, \mathrm{Arg}^{506}$ and $\mathrm{Arg}^{679}$ in the heavy chain, subsequently resulting in a dissociation of the $\mathrm{A} 2$ domain ${ }^{122,123}$. (Figure 8). It was demonstrated that the eastern brown snake (Pseudonaja textilis) has a FV-like protein (ptFV) derived from its venom which is functionally resistant to inactivation by APC cleavage ${ }^{124-126}$. By structural and functional investigation of both human FVa and ptFVa, the mechanistic principles of the exceptional procoagulant evolution of ptFVa were elucidated. Furthermore, these investigations guide us in the design of novel FV molecules for the treatment of bleeding disorders. 


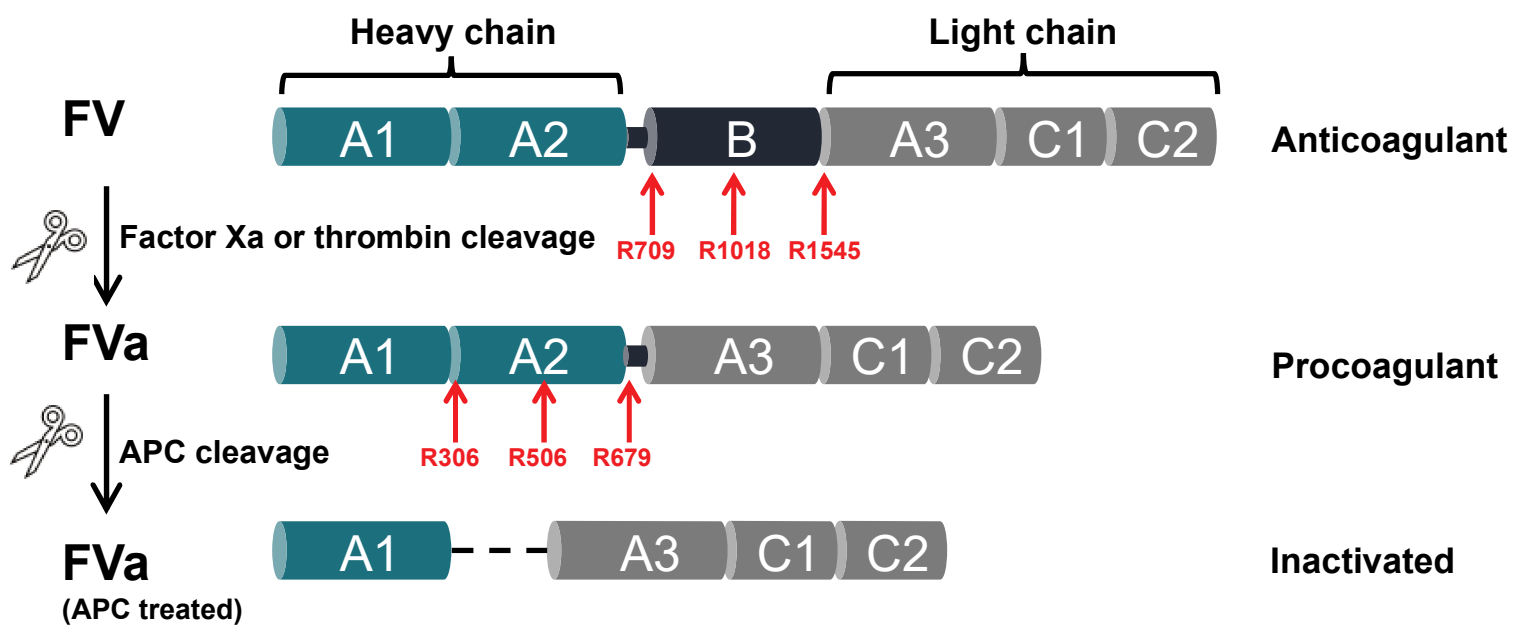

Figure 8. The activation and inactivation of human coagulation factor $V(F V)$. Shown are the domain organization and specific cleavage sites for factor $\mathrm{Xa}$, thrombin and activated protein $C$ (APC). FV is a single chain plasma protein, whereas activated FV (FVa) is a heterodimer.

\subsubsection{The C-X-C chemokine receptor type 4 (CXCR4)}

The chemokine receptor CXCR4 is mainly expressed on the surface of white blood cells, including neutrophils, monocytes, eosinophil, T-lymphocytes, B cells, and natural killer cells. CXCR4 is activated by binding to its chemokine ligand CXCL12 (stromal cell-derived factor 1alpha), which controls homing and trafficking of hematopoietic stem and progenitor cells (HSPCs) $)^{127-129}$. The positive role of the CXCR4/CXCL12 axis in myocardial ischemia/reperfusion injury was discovered by experiments through which it was shown that CXCL12 protects cardiac muscle cells and recruits stem cells to damaged cardiac sites after myocardial infarction ${ }^{130,131}$. Deletion of CXCR4 aggravated loss of cardiac function in an animal model of heart failure ${ }^{132}$. Recently, it was demonstrated that the CXCR4/CXCL12 axis antagonizes neutrophil diurnal aging, a process that controls how and when neutrophils migrate into tissues and that is regulated by CXCR2/CXCL2 signaling (Figure 9). Increasing the numbers of circulating aged neutrophils by CXCR4 depletion increased neutrophil extravasation into tissues and protected against infections. On the other hand, the increase of circulating aged neutrophils enhanced the thrombo-inflammatory response with increased vascular inflammation following myocardial ischemia/reperfusion injury ${ }^{133}$ (Figure 9). Taken together, regulation of CXCR4 signaling may represent a novel therapeutic avenue to treat patients susceptible to infections (CXCR4 antagonists) and patients at risk of cardiovascular events (CXCR4 agonists). 


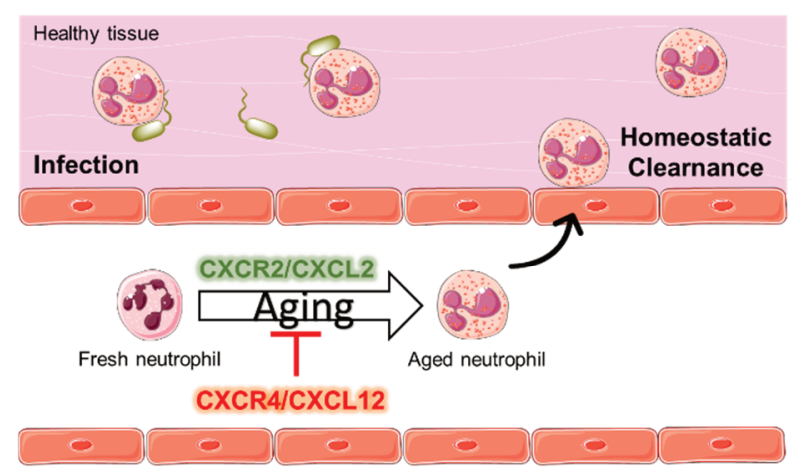

Healthy model

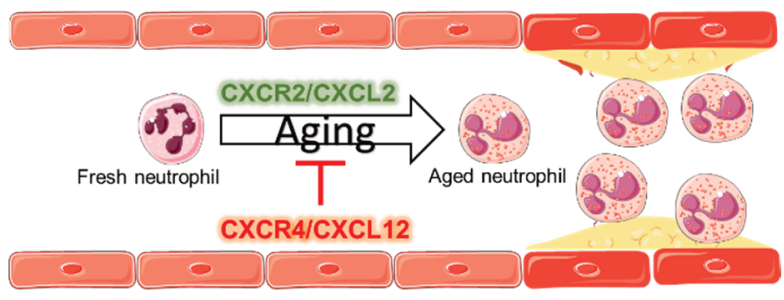

myocardial ischemia/reperfusion model

Figure 9. The role of aged neutrophils in healthy and myocardial ischemia/reperfusion model. Neutrophil aging process is promoted by CXCR2/CXCL2 axis, aged neutrophils can infiltrate to non-inflamed tissues. This process contributes to neutrophil homeostasis by reducing the number of neutrophils in blood as well as by enhancing immune defence in healthy model. However, the increase of circulating aged neutrophils in blood can significantly cause the vascular damage in mice model of acute myocardial infarction, and even cause thrombus formation in CXCR4 knockout myocardial ischemia/ reperfusion mice mode/ ${ }^{133}$.

\subsection{Outline of this thesis}

This thesis focuses on the application of structural bioinformatics approaches to expedite and focus our understanding of various therapeutic target proteins involved in cardiovascular diseases and to identify novel molecules that may interfere with or modify the interactions of those proteins.

In Chapter $\mathbf{2}$ the rational design of modulators (e.g., inhibitors or stabilizers) by targeting protein-protein interactions (PPIs) is introduced. We described both experimental and computational methods for the determination of the $3 \mathrm{D}$ structure of protein-protein complexes, as well as computational approaches that have been applied to design modulators based on PPIs.

In Chapter 3 we summarize the latest information on PAD4, including its structure, its physiological functions and roles in various pathologies, as well as its regulators for activation. Moreover, known PAD4 inhibitors, including their advantages and limitations, are reported and discussed in this chapter.

In Chapter 4 we investigate the autocitrullination of PAD4 in greater detail, as it remains unclear whether this process serves as a novel mechanism to regulate PAD4 activity. To address this, we performed in vitro approaches to evaluate both the activity of PAD4 and citrullinated PAD4, whilst employing in silico approaches to reveal the structural stability of both PAD4 and citrullinated PAD4 and their binding to substrates.

In Chapter $\mathbf{5}$ the procoagulant activity of human FVa is downregulated by APC cleavage, which results in a dissociation of the $\mathrm{A} 2$ domain. It has been shown that the homologous snake (eastern brown snake) venom FVa shows functional resistance to APC cleavage. Based on the structural differences between human and snake venom $\mathrm{FVa}$, we aim to elucidate the mechanistic principles of the activity resistance of snake FVa to APC by using both in vitro and in silico approaches.

In Chapter $\mathbf{6}$ we apply computational methods to design a peptide agonist that can activate CXCR4, as it was reported that CXCR4 together with its natural agonist CXCL12 antagonize the neutrophil aging 
process where aged neutrophils contribute to vascular damage in ischemia reperfusion model. Two strategies are deployed with one strategy using a known allosteric pepducin as a template, targeting CXCR4 intracellularly, and another strategy using the natural agonist CXCL12 as a template, targeting CXCR4 extracellularly. The designed peptides were then synthesized and subjected to activity assessment through in vitro experiments.

In Chapter 7 we discuss all the results presented in this thesis and provide a future perspective. 


\section{References}

1. Mullard, A., New drugs cost US\$2.6 billion to develop. Nature Reviews Drug Discovery 2014, 13 (12), 877 877.

2. Dickson, M.; Gagnon, J. P., The cost of new drug discovery and development. Discov Med 2004, 4 (22), 172-9.

3. Wouters, O. J., et al., Estimated Research and Development Investment Needed to Bring a New Medicine to Market, 2009-2018. JAMA 2020, 323 (9), 844-853.

4. Schneider, G.; Fechner, U., Computer-based de novo design of drug-like molecules. Nature Reviews Drug Discovery 2005, 4 (8), 649-663.

5. Xiang, Z., Advances in homology protein structure modeling. Current protein \& peptide science 2006, 7 (3), 217-227.

6. Muhammed, M. T.; Aki-Yalcin, E., Homology modeling in drug discovery: Overview, current applications, and future perspectives. Chemical Biology \& Drug Design 2019, 93 (1), 12-20.

7. Mosimann, S., et al., A critical assessment of comparative molecular modeling of tertiary structures of proteins. Proteins 1995, 23 (3), 301-17.

8. Sauder, J. M., et al., Large-scale comparison of protein sequence alignment algorithms with structure alignments. Proteins 2000, 40 (1), 6-22.

9. McGinnis, S.; Madden, T. L., BLAST: at the core of a powerful and diverse set of sequence analysis tools. Nucleic Acids Research 2004, 32 (suppl_2), W20-W25.

10. Taylor, W. R., Identification of protein sequence homology by consensus template alignment. Journal of Molecular Biology 1986, 188 (2), 233-258.

11. Geourjon, C., et al., Identification of related proteins with weak sequence identity using secondary structure information. Protein science : a publication of the Protein Society 2001, 10 (4), 788-797.

12. Land, H.; Humble, M. S., YASARA: A Tool to Obtain Structural Guidance in Biocatalytic Investigations. Methods Mol Biol 2018, 1685, 43-67.

13. Eswar, N., et al., Comparative protein structure modeling using Modeller. Curr Protoc Bioinformatics 2006, Chapter 5, Unit-5.6.

14. Waterhouse, A., et al., SWISS-MODEL: homology modelling of protein structures and complexes. Nucleic Acids Research 2018, 46 (W1), W296-W303.

15. Yang, J., et al., The I-TASSER Suite: protein structure and function prediction. Nat Methods 2015, 12 (1), 7-8.

16. Rodriguez, R., et al., Homology modeling, model and software evaluation: three related resources. Bioinformatics 1998, 14 (6), 523-8.

17. Thornton, R. A. L. W. M. S. M. T. J., PROCHECK: A program to check the stereochemical quality of protein structures. Journal of Applied Crystallography 1993, 26 (2), 283-291.

18. Wiederstein, M.; Sippl, M. J., ProSA-web: interactive web service for the recognition of errors in threedimensional structures of proteins. Nucleic acids research 2007, 35 (Web Server issue), W407-W410.

19. Chen, V. B., et al., MolProbity: all-atom structure validation for macromolecular crystallography. Acta Crystallogr D Biol Crystallogr 2010, 66 (Pt 1), 12-21.

20. Weichenberger, C. X.; Sippl, M. J., NQ-Flipper: recognition and correction of erroneous asparagine and glutamine side-chain rotamers in protein structures. Nucleic Acids Research 2007, 35 (suppl_2), W403W406.

21. Lionta, E., et al., Structure-based virtual screening for drug discovery: principles, applications and recent advances. Curr Top Med Chem 2014, 14 (16), 1923-1938.

22. Joosten RP, e. a. a. V. G., PDB_REDO: automated re-refinement of X-ray structure models in the PDB. J. Appl. Cryst. 2009, 42, 376-384.

23. consortium, P. D.-K., PDBe-KB: a community-driven resource for structural and functional annotations. Nucleic Acids Research 2020, 48 (D1), D344-D353.

24. Meng, X.-Y., et al., Molecular docking: a powerful approach for structure-based drug discovery. Curr Comput Aided Drug Des 2011, 7 (2), 146-157.

25. Bento, A. P., et al., The ChEMBL bioactivity database: an update. Nucleic Acids Res 2014, 42 (Database issue), D1083-90.

26. Li, Q., et al., PubChem as a public resource for drug discovery. Drug Discov Today 2010, 15 (23-24), 1052-7.

27. Wishart, D. S., et al., DrugBank: a comprehensive resource for in silico drug discovery and exploration. Nucleic Acids Res 2006, 34 (Database issue), D668-72.

28. Methods for the Prediction of Protein-Ligand Binding Sites for Structure-Based Drug Design and Virtual Ligand Screening. Current Protein \& Peptide Science 2006, 7 (5), 395-406.

29. Berman, H. M., et al., The Protein Data Bank. Nucleic acids research 2000, 28 (1), 235-242. 
30. Le Guilloux, V., et al., Fpocket: An open source platform for ligand pocket detection. BMC Bioinformatics 2009, 10 (1), 168.

31. Hernandez, M., et al., SITEHOUND-web: a server for ligand binding site identification in protein structures. Nucleic Acids Research 2009, 37, W413 - W416.

32. Brylinski, M.; Feinstein, W. P., eFindSite: improved prediction of ligand binding sites in protein models using meta-threading, machine learning and auxiliary ligands. J Comput Aided Mol Des 2013, 27 (6), 55167.

33. Gao, J., et al., bSiteFinder, an improved protein-binding sites prediction server based on structural alignment: more accurate and less time-consuming. Journal of Cheminformatics 2016, 8 (1), 38.

34. Krivák, R.; Hoksza, D., P2Rank: machine learning based tool for rapid and accurate prediction of ligand binding sites from protein structure. Journal of Cheminformatics 2018, 10 (1), 39.

35. Lagorce, D., et al., FAF-Drugs4: free ADME-tox filtering computations for chemical biology and early stages drug discovery. Bioinformatics 2017, 33 (22), 3658-3660.

36. Baell, J. B.; Holloway, G. A., New Substructure Filters for Removal of Pan Assay Interference Compounds (PAINS) from Screening Libraries and for Their Exclusion in Bioassays. Journal of Medicinal Chemistry 2010, 53 (7), 2719-2740.

37. Tsaioun, K., ADME Properties Leading to Toxicity. Drug Discovery Toxicology 2016, 82-92.

38. Lipinski, C. A., et al., Experimental and computational approaches to estimate solubility and permeability in drug discovery and development settings1PII of original article: S0169-409X(96)00423-1. The article was originally published in Advanced Drug Delivery Reviews 23 (1997) 3-25.1. Advanced Drug Delivery Reviews 2001, 46 (1), 3-26.

39. Irwin, J. J., et al., ZINC: A Free Tool to Discover Chemistry for Biology. Journal of Chemical Information and Modeling 2012, 52 (7), 1757-1768.

40. Liu, T., et al., BindingDB: a web-accessible database of experimentally determined protein-ligand binding affinities. Nucleic Acids Res 2007, 35 (Database issue), D198-201.

41. Hawkins, P. C. D., et al., Conformer generation with OMEGA: algorithm and validation using high quality structures from the Protein Databank and Cambridge Structural Database. Journal of chemical information and modeling 2010, 50 (4), 572-584.

42. O'Boyle, N. M., et al., Open Babel: An open chemical toolbox. Journal of Cheminformatics 2011, 3 (1), 33.

43. Watts, K. S., et al., ConfGen: a conformational search method for efficient generation of bioactive conformers. J Chem Inf Model 2010, 50 (4), 534-46.

44. Lovrić, M., et al., PySpark and RDKit: Moving towards Big Data in Cheminformatics. Mol Inform 2019, 38 (6), e1800082.

45. Kitchen, D. B., et al., Docking and scoring in virtual screening for drug discovery: methods and applications. Nature Reviews Drug Discovery 2004, 3 (11), 935-949.

46. Huang, S. Y., et al., Scoring functions and their evaluation methods for protein-ligand docking: recent advances and future directions. Phys Chem Chem Phys 2010, 12 (40), 12899-908.

47. Guedes, I. A., et al., Empirical Scoring Functions for Structure-Based Virtual Screening: Applications, Critical Aspects, and Challenges. Frontiers in Pharmacology 2018, 9, 1089.

48. Muegge, I., PMF Scoring Revisited. Journal of Medicinal Chemistry 2006, 49 (20), 5895-5902.

49. Kinnings, S. L., et al., A Machine Learning-Based Method To Improve Docking Scoring Functions and Its Application to Drug Repurposing. Journal of Chemical Information and Modeling 2011, 51 (2), 408-419.

50. McGann, M., FRED pose prediction and virtual screening accuracy. J Chem Inf Model 2011, 51 (3), 57896.

51. Jain, A. N., Surflex: fully automatic flexible molecular docking using a molecular similarity-based search engine. J Med Chem 2003, 46 (4), 499-511.

52. Friesner, R. A., et al., Glide: a new approach for rapid, accurate docking and scoring. 1. Method and assessment of docking accuracy. J Med Chem 2004, 47 (7), 1739-49.

53. Allen, W. J., et al., DOCK 6: Impact of new features and current docking performance. J Comput Chem 2015, 36 (15), 1132-1156.

54. Trott, O.; Olson, A. J., AutoDock Vina: improving the speed and accuracy of docking with a new scoring function, efficient optimization, and multithreading. J Comput Chem 2010, 31 (2), 455-461.

55. Neves, M. A. C., et al., Docking and scoring with ICM: the benchmarking results and strategies for improvement. J Comput Aided Mol Des 2012, 26 (6), 675-686.

56. Verdonk, M. L., et al., Improved protein-ligand docking using GOLD. Proteins 2003, 52 (4), 609-23.

57. Du, J., et al., Optimization of Compound Ranking for Structure-Based Virtual Ligand Screening Using an Established FRED-Surflex Consensus Approach. Chemical Biology \& Drug Design 2014, 83 (1), $37-51$.

58. Perola, E., Minimizing false positives in kinase virtual screens. Proteins 2006, 64 (2), 422-35.

59. Adeshina, Y. O., et al., Machine learning classification can reduce false positives in structure-based virtual screening. Proceedings of the National Academy of Sciences 2020, 117 (31), 18477. 
60. Cozzini, P., et al., Target flexibility: an emerging consideration in drug discovery and design. J Med Chem 2008, 51 (20), 6237-55.

61. Cavasotto, C. N.; Abagyan, R. A., Protein flexibility in ligand docking and virtual screening to protein kinases. J Mol Biol 2004, 337 (1), 209-25.

62. Amaro, R. E., et al., Ensemble Docking in Drug Discovery. Biophys J 2018, 114 (10), 2271-2278.

63. Graves, A. P., et al., Rescoring Docking Hit Lists for Model Cavity Sites: Predictions and Experimental Testing. Journal of Molecular Biology 2008, 377 (3), 914-934.

64. Huang, N., et al., Physics-Based Scoring of Protein-Ligand Complexes: Enrichment of Known Inhibitors in Large-Scale Virtual Screening. Journal of Chemical Information and Modeling 2006, 46 (1), 243-253.

65. Kukol, A., Consensus virtual screening approaches to predict protein ligands. European Journal of Medicinal Chemistry 2011, 46 (9), 4661-4664.

66. Charifson, P. S., et al., Consensus Scoring: A Method for Obtaining Improved Hit Rates from Docking Databases of Three-Dimensional Structures into Proteins. Journal of Medicinal Chemistry 1999, 42 (25), 5100-5109.

67. Ericksen, S. S., et al., Machine Learning Consensus Scoring Improves Performance Across Targets in Structure-Based Virtual Screening. Journal of Chemical Information and Modeling 2017, 57 (7), 1579-1590.

68. Amaro, R. E., et al., An improved relaxed complex scheme for receptor flexibility in computer-aided drug design. J Comput Aided Mol Des 2008, 22 (9), 693-705.

69. Lin, J. H., et al., The relaxed complex method: Accommodating receptor flexibility for drug design with an improved scoring scheme. Biopolymers 2003, 68 (1), 47-62.

70. Lin, J.-H., et al., Computational Drug Design Accommodating Receptor Flexibility: The Relaxed Complex Scheme. Journal of the American Chemical Society 2002, 124 (20), 5632-5633.

71. Zarbafian, S., et al., Protein docking refinement by convex underestimation in the low-dimensional subspace of encounter complexes. Sci Rep 2018, 8 (1), 5896.

72. Hou, T., et al., Assessing the performance of the molecular mechanics/Poisson Boltzmann surface area and molecular mechanics/generalized Born surface area methods. II. The accuracy of ranking poses generated from docking. $J$ Comput Chem 2011, 32 (5), 866-77.

73. $\mathrm{Xu}$, L., et al., Assessing the performance of MM/PBSA and MM/GBSA methods. 3. The impact of force fields and ligand charge models. J Phys Chem B 2013, 117 (28), 8408-21.

74. Brown, S. P.; Muchmore, S. W., Large-Scale Application of High-Throughput Molecular Mechanics with Poisson-Boltzmann Surface Area for Routine Physics-Based Scoring of Protein-Ligand Complexes. Journal of Medicinal Chemistry 2009, 52 (10), 3159-3165.

75. Zhang, X., et al., Toward Fully Automated High Performance Computing Drug Discovery: A Massively Parallel Virtual Screening Pipeline for Docking and Molecular Mechanics/Generalized Born Surface Area Rescoring to Improve Enrichment. Journal of Chemical Information and Modeling 2014, 54 (1), 324-337.

76. Greenidge, P. A., et al., Improving docking results via reranking of ensembles of ligand poses in multiple X-ray protein conformations with MM-GBSA. J Chem Inf Model 2014, 54 (10), 2697-717.

77. Zhang, X., et al., A Comprehensive Docking and MM/GBSA Rescoring Study of Ligand Recognition upon Binding Antithrombin. Curr Top Med Chem 2017, 17 (14), 1631-1639.

78. Karplus, M.; McCammon, J. A., Molecular dynamics simulations of biomolecules. Nature Structural Biology 2002, 9 (9), 646-652.

79. Pikkemaat, M. G., et al., Molecular dynamics simulations as a tool for improving protein stability. Protein Engineering, Design and Selection 2002, 15 (3), 185-192.

80. Aier, I., et al., Structural insights into conformational stability of both wild-type and mutant EZH2 receptor. Sci Rep 2016, 6 (1), 34984.

81. Khan, S., et al., Protein stability and dynamics influenced by ligands in extremophilic complexes - a molecular dynamics investigation. Molecular BioSystems 2017, 13 (9), 1874-1887.

82. Guterres, H., et al., Ligand-Binding-Site Structure Refinement Using Molecular Dynamics with Restraints Derived from Predicted Binding Site Templates. Journal of chemical theory and computation 2019, 15 (11), 6524-6535.

83. Daura, X., et al., Reversible peptide folding in solution by molecular dynamics simulation11Edited by $\mathrm{R}$. Huber. Journal of Molecular Biology 1998, 280 (5), 925-932.

84. Duan, Y., et al., The early stage of folding of villin headpiece subdomain observed in a 200-nanosecond fully solvated molecular dynamics simulation. Proceedings of the National Academy of Sciences 1998, 95 (17), 9897.

85. Cui, Q., et al., Molecular Dynamics—Solvated Interaction Energy Studies of Protein-Protein Interactions: The MP1-p14 Scaffolding Complex. Journal of Molecular Biology 2008, 379 (4), 787-802.

86. Liu, K., et al., Exploring the stability of ligand binding modes to proteins by molecular dynamics simulations. J Comput Aided Mol Des 2017, 31 (2), 201-211.

87. Åqvist, J., et al., Ligand Binding Affinities from MD Simulations. Accounts of Chemical Research 2002, 35 (6), 358-365. 
88. Lu, S.-Y., et al., Molecular docking and molecular dynamics simulation studies of GPR40 receptor-agonist interactions. Journal of Molecular Graphics and Modelling 2010, 28 (8), 766-774.

89. Durrant, J. D.; McCammon, J. A., Molecular dynamics simulations and drug discovery. BMC Biology 2011, 9 (1), 71.

90. Brooks, B. R., et al., CHARMM: A program for macromolecular energy, minimization, and dynamics calculations. J Comput Chem 1983, 4 (2), 187-217.

91. Case, D. A., et al., The Amber biomolecular simulation programs. J Comput Chem 2005, 26 (16), 166888.

92. Pronk, S., et al., GROMACS 4.5: a high-throughput and highly parallel open source molecular simulation toolkit. Bioinformatics 2013, 29 (7), 845-54.

93. Jorgensen, W. L.; Thomas, L. L., Perspective on Free-Energy Perturbation Calculations for Chemical Equilibria. Journal of chemical theory and computation 2008, 4 (6), 869-876.

94. Bauß, A., et al., Thermodynamic Integration Networks and Their Application to Charge Transfer Reactions within the AauDyPI Fungal Peroxidase. The Journal of Physical Chemistry B 2016, 120 (22), 4937-4944.

95. Rifai, E. A., et al., Recent Developments in Linear Interaction Energy Based Binding Free Energy Calculations. Frontiers in Molecular Biosciences 2020, 7 (114).

96. Wang, E., et al., End-Point Binding Free Energy Calculation with MM/PBSA and MM/GBSA: Strategies and Applications in Drug Design. Chemical Reviews 2019, 119 (16), 9478-9508.

97. Genheden, S.; Ryde, U., The MM/PBSA and MM/GBSA methods to estimate ligand-binding affinities. Expert Opin Drug Discov 2015, 10 (5), 449-461.

98. Gilson, M. K.; Honig, B., Calculation of the total electrostatic energy of a macromolecular system: solvation energies, binding energies, and conformational analysis. Proteins 1988, 4 (1), 7-18.

99. Attard, P., et al., Information content of signals using correlation function expansions of the entropy. Physical Review E 1997, 56 (4), 4052-4067.

100. Ghoufi, A.; Malfreyt, P., Entropy and enthalpy calculations from perturbation and integration thermodynamics methods using molecular dynamics simulations: applications to the calculation of hydration and association thermodynamic properties. Molecular Physics 2006, 104 (18), 2929-2943.

101. Bicker, K. L.; Thompson, P. R., The protein arginine deiminases: Structure, function, inhibition, and disease. Biopolymers 2013, 99 (2), 155-163.

102. Li, P., et al., PAD4 is essential for antibacterial innate immunity mediated by neutrophil extracellular traps. J Exp Med 2010, 207 (9), 1853-1862.

103. Lewis, H. D., et al., Inhibition of PAD4 activity is sufficient to disrupt mouse and human NET formation. Nat Chem Biol 2015, 11 (3), 189-191.

104. Rohrbach, A. S., et al., Activation of PAD4 in NET formation. Frontiers in immunology 2012, 3, 360-360.

105. Martinod, K., et al., Neutrophil histone modification by peptidylarginine deiminase 4 is critical for deep vein thrombosis in mice. Proc Natl Acad Sci U S A 2013, 110 (21), 8674-8679.

106. Kimball, A. S., et al., The Emerging Role of NETs in Venous Thrombosis and Immunothrombosis. Frontiers in Immunology 2016, 7, 236.

107. Silvestre-Roig, C., et al., Externalized histone $\mathrm{H} 4$ orchestrates chronic inflammation by inducing lytic cell death. Nature 2019, 569 (7755), 236-240.

108. Wildhagen, K. C., et al., Extracellular histone $\mathrm{H} 3$ levels are inversely correlated with antithrombin levels and platelet counts and are associated with mortality in sepsis patients. Thromb Res 2015, 136 (3), 5427.

109. Zhou, Y., et al., Spontaneous Secretion of the Citrullination Enzyme PAD2 and Cell Surface Exposure of PAD4 by Neutrophils. Frontiers in Immunology 2017, 8, 1200.

110. Spengler, J., et al., Release of Active Peptidyl Arginine Deiminases by Neutrophils Can Explain Production of Extracellular Citrullinated Autoantigens in Rheumatoid Arthritis Synovial Fluid. Arthritis Rheumatol 2015, 67 (12), 3135-45.

111. Sorvillo, N., et al., Plasma Peptidylarginine Deiminase IV Promotes VWF-Platelet String Formation and Accelerates Thrombosis after Vessel Injury. Circulation research 2019.

112. Ordóñez, A., et al., Effect of citrullination on the function and conformation of antithrombin. The FEBS Journal 2009, 276 (22), 6763-6772.

113. Elisabeth Thorelli, R. J. K., and Bjorn Dahlback, The C-terminal Region of the Factor V B-domain Is Crucial for the Anticoagulant Activity of Factor V*. The Journal of Biological Chemistry 1998, 273 (26), 1641016415.

114. Cramer, T. J., et al., Factor $\mathrm{V}$ is an anticoagulant cofactor for activated protein $\mathrm{C}$ during inactivation of factor Va. Pathophysiol Haemost Thromb 2010, 37 (1), 17-23.

115. Monkovic, D. D.; Tracy, P. B., Activation of human factor $V$ by factor Xa and thrombin. Biochemistry 1990, 29 (5), 1118-1128.

116. Nicolaes, G. A.; Dahlbäck, B., Factor $V$ and thrombotic disease: description of a janus-faced protein. Arterioscler Thromb Vasc Biol 2002, 22 (4), 530-8. 
117. Tans, G., et al., Activation of human factor V by meizothrombin. J Biol Chem 1994, 269 (23), 15969-72.

118. Rosing, J., et al., The role of phospholipids and factor Va in the prothrombinase complex. J Biol Chem 1980, 255 (1), 274-83.

119. Castoldi, E., et al., Impaired APC cofactor activity of factor $\mathrm{V}$ plays a major role in the APC resistance associated with the factor V Leiden (R506Q) and R2 (H1299R) mutations. Blood 2004, 103 (11), 4173-9.

120. DahlbäCk, B., Factor $V$ gene mutation causing inherited resistance to activated protein $\mathrm{C}$ as a basis for venous thromboembolism. Journal of Internal Medicine 1995, 237 (3), 221-227.

121. Bertina, R. M., et al., Mutation in blood coagulation factor $V$ associated with resistance to activated protein C. Nature 1994, 369 (6475), 64-67.

122. Mann, K. G., et al., Activated protein C cleavage of factor Va leads to dissociation of the A2 domain. J Biol Chem 1997, 272 (33), 20678-83.

123. Gerry A. F. Nicolaes, G. T., M. Christella L. G. D. Thomassen, H. Coenraad Hemker, Ingrid Pabinger, Katalin Varadi, Hans P. Schwarz and Jan Rosing, Peptide Bond Cleavages and Loss of Functional Activity during Inactivation of Factor Va and Factor VaR506Q by Activated Protein C The Journal of Biological Chemistry 1995, 270, 21158-21166.

124. Rao, V. S., et al., The nonenzymatic subunit of pseutarin C, a prothrombin activator from eastern brown snake (Pseudonaja textilis) venom, shows structural similarity to mammalian coagulation factor V. Blood 2003, 102 (4), 1347-1354.

125. Bos, M. H., et al., Venom factor $V$ from the common brown snake escapes hemostatic regulation through procoagulant adaptations. Blood 2009, 114 (3), 686-92.

126. Verhoef, D., et al., Functional implications of the unique disulfide bond in venom factor $V$ from the Australian common brown snake Pseudonaja textilis. Toxin Reviews 2014, 33 (1-2), 37-41.

127. Devine, S. M., et al., Rapid mobilization of functional donor hematopoietic cells without G-CSF using AMD3100, an antagonist of the CXCR4/SDF-1 interaction. Blood 2008, 112 (4), 990-8.

128. Tzeng, Y. S., et al., Loss of Cxcl12/Sdf-1 in adult mice decreases the quiescent state of hematopoietic stem/progenitor cells and alters the pattern of hematopoietic regeneration after myelosuppression. Blood 2011, 117 (2), 429-39.

129. Karpova, D., et al., Continuous blockade of CXCR4 results in dramatic mobilization and expansion of hematopoietic stem and progenitor cells. Blood 2017, 129 (21), 2939-2949.

130. Hu, X., et al., Stromal cell derived factor-1 alpha confers protection against myocardial ischemia/reperfusion injury: role of the cardiac stromal cell derived factor- 1 alpha CXCR4 axis. Circulation 2007, 116 (6), 654-63.

131. Saxena, A., et al., Stromal cell-derived factor-1alpha is cardioprotective after myocardial infarction. Circulation 2008, 117 (17), 2224-31.

132. Wang, E. R., et al., Deletion of CXCR4 in cardiomyocytes exacerbates cardiac dysfunction following isoproterenol administration. Gene Ther 2014, 21 (5), 496-506.

133. Adrover, J. M., et al., A Neutrophil Timer Coordinates Immune Defense and Vascular Protection. Immunity 2019, 50 (2), 390-402.e10. 



\section{Chapter}

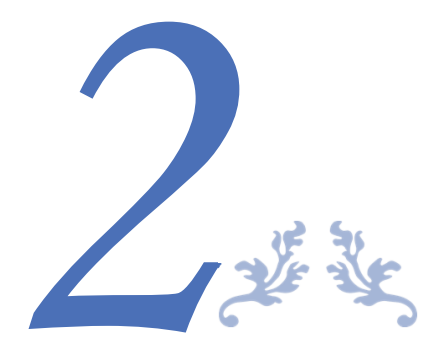

\section{Rational modulator design by exploitation of protein-protein complex structures}

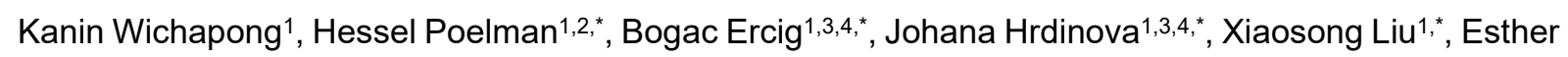
Lutgens $^{2,5}$, and Gerry A. F. Nicolaes ${ }^{1,2}$,

1Department of Biochemistry, Cardiovascular Research Institute Maastricht (CARIM), Maastricht University, Maastricht, The Netherlands

2Department of Medical Biochemistry, Amsterdam Medical Center, Amsterdam, The Netherlands

${ }^{3}$ Department of Plasma Proteins, Sanquin-AMC Landsteiner Laboratory, Amsterdam, The Netherlands ${ }^{4}$ PharmaTarget B.V. Maastricht, The Netherlands

${ }^{5}$ Institute for Cardiovascular Prevention, Ludwig-Maximilians University Munich, Munich, Germany

*These authors contributed equally 


\begin{abstract}
The horizon of drug discovery is currently expanding to target and modulate protein-protein interactions (PPIs) in globular proteins and intrinsically disordered proteins (IDPs) that are involved in various diseases. To either interrupt or stabilize PPIs, the 3D structure of target protein-protein (or proteinpeptide) complexes can be exploited to rationally design PPI modulators (inhibitors or stabilizers) through structure-based molecular design. In this review, we present an overview of experimental and computational methods that can be used to determine 3D structures of protein-protein complexes. Several approaches including rational and in silico methods that can be applied to design peptides, peptidomimetics, and small compounds by utilization of determined 3D protein-protein/peptide complexes are summarized and illustrated.
\end{abstract}

Keywords: Protein-protein interactions (PPIs), PPI Modulators, Peptide Design, Peptidomimetics, Intrinsically Disordered Proteins (IDPs) 


\section{$2.1 \quad$ Introduction}

Protein-protein interactions (PPIs) are at the very basis of function in biological systems as they essentially connect the players that are involved and perform important roles in virtually all biological pathways, including signal transduction, immune recognition, transmembrane transport, cell-cell interactions, posttranslational modification, and maintenance of cellular organization ${ }^{1}$. Inevitably, reflecting their pivotal importance, PPIs are involved in the etiology of numerous diseases e.g. cancer, infectious diseases, inflammatory diseases, neurodegenerative diseases and autoimmune disease ${ }^{2-6}$. Despite the recognized importance of proteins as target for pharmacotherapy, PPI's per se have long been neglected as potential drug targets for targeting by drug-like inhibitors, mainly due to the supposedly undruggable flat and large interfaces between the protein partners in the protein-protein complexes. PPI interfaces vary from 1,000 to $3,000 \mathrm{~A}^{2}$ whereas typical binding pockets in proteins are comparatively small (around 300-1,000 $\left.A^{2}\right)^{7-9}$. However, the PPI research field is undergoing rapid development and receives growing interest as evidenced by the fact that during the last 20 years novel peptides, peptidomimetics, and small compounds have been increasingly discovered and developed to inhibit or stabilize PPIs ${ }^{7}, 10-13$ The number of PPI modulators is likely to increase, considering that only a small proportion of the PPIs from the estimated 650,000 different protein-protein complexes identified in the human interactome have been targeted through drug discovery ${ }^{9}, 14$. Thus, there are still vast numbers of PPIs that have not yet been explored and targeted for drug development. Not only welldefined protein-protein surfaces are being targeted, recently, the horizon of drug discovery research field has been expanded to target also unstructured proteins that play essential roles in PPIs. Development of novel inhibitors binding to intrinsically disordered proteins (IDP) regions (IDPR) such as in C-Myc ${ }^{15}{ }^{16}$, amyloid- $\beta$ peptide ${ }^{17}$, and histone ${ }^{18}$ are examples to prove the concept of IDPs and IDPRs as being druggable and promising targets. Another example is compound MSI-1436 which binds to the disorder $\mathrm{C}$ terminus of PTP1B. This compound is now entering clinical trial phases for treating breast cancer, obesity, and diabetes ${ }^{19,} 20$ confirming the principle of concept that targeting disordered proteins is feasible. Therefore, development of small compounds or peptides to target either the interfaces or the unstructured parts of protein binding partners, in order to interrupt or stabilize PPIs, represents a promising future trend in drug discovery research.

Although inhibitors and stabilizers of PPIs can bind at the proximal subpockets at the interface such as in the case of 14-3-3 protein ${ }^{21}$ and it seems that they likely have common features for binding and interacting with protein-protein complexes, recent studies have shown that small molecule stabilizers possess different physiochemical properties than inhibitors ${ }^{11,}{ }^{22}$. Nevertheless, despite the heterogeneity in the small molecules themselves, similar common approaches can be applied to develop PPI modulators, being either inhibitors or stabilizers. PPI modulators can be subdivided in two different classes, depending on the type of interface involved. Modulation of PPI's can be done by development of compounds binding at an orthosteric interface (a pocket located between the interfaces of two proteins) or at an allosteric pocket (a distant pocket from the interface of two interacting proteins) $)^{2,23,24}$ as shown in Figure 1. Orthosteric modulators regulate PPIs either by functioning as inhibitors (iPPI) to disrupt PPIs to prevent complex formation between two proteins or, on the other hand 
stabilizers (sPPI), they have different roles by functioning as molecular glue to connect and stabilize two proteins which can consequently promote the function of protein heteromers.. In contrast, allosteric modulators control PPIs by binding at the allosteric pocket which subsequently can induce a conformational change of the binding proteins, resulting in the prevention (iPPI) or enhancement (sPPI) of protein-protein interactions. Targeting allosteric pockets is attractive, as large molecules may not be required for binding an allosteric pocket in order to change the protein conformation but this strategy still remains a non-trivial task ${ }^{24}, 25$. In contrast, the orthosteric protein interface provides interaction information of the proteins involved which can be more readily utilized to rationally design orthosteric modulators mimicking these critical interactions ${ }^{24}$.

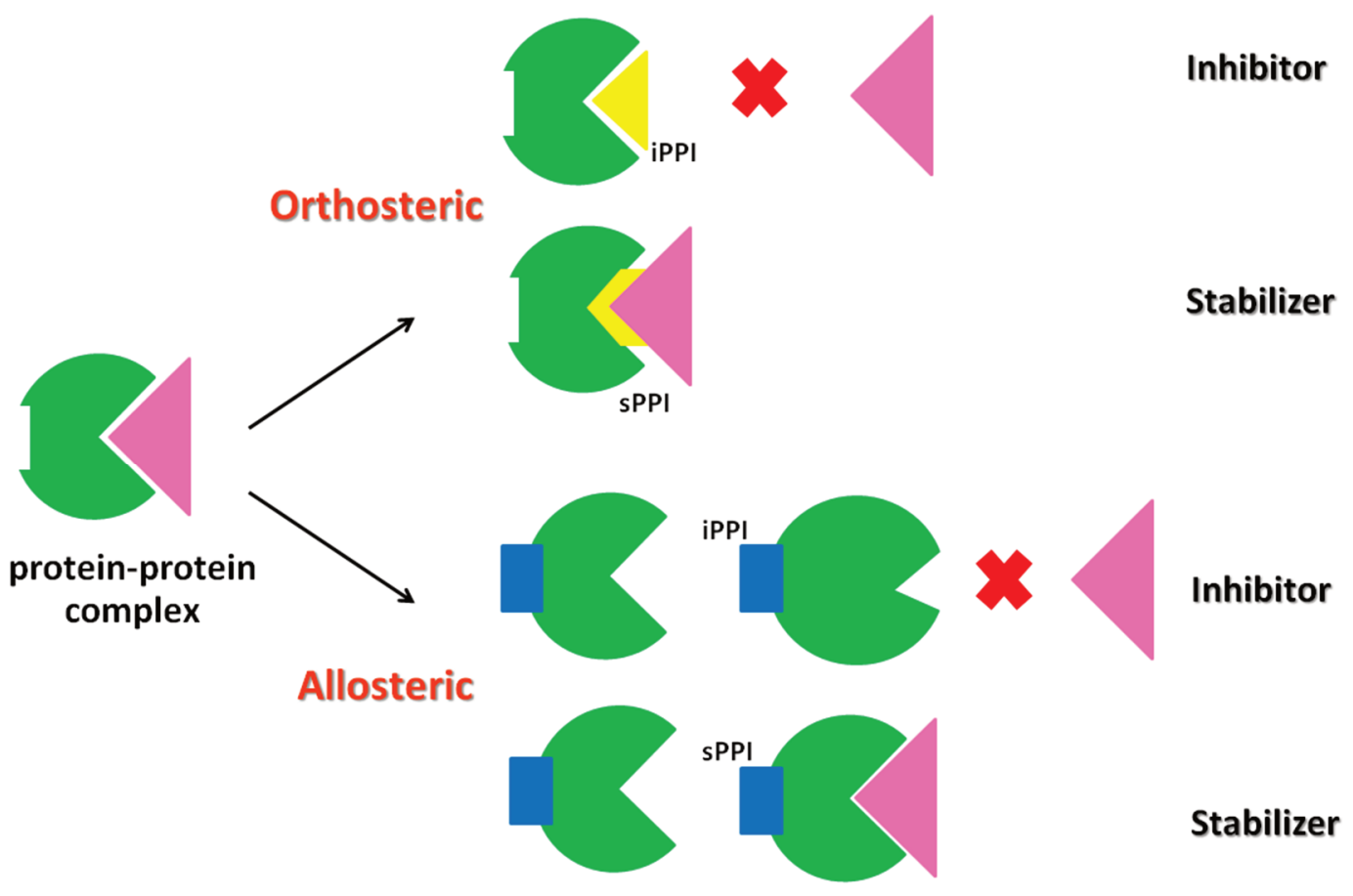

Figure 1. Modulation of PPIs. Inhibitors (iPPI) or stabilizers (sPPI) target PPIs at the orthosteric or allosteric pocket $^{2,23,24}$.

Next to a division based on the property of the interfaces, PPIs can also be divided into different structural classes depending on the type of binding partner involved. Thus we can distinguish PPIs formed by complexation of two globular proteins, by binding of a single peptide chain, disordered proteins or unstructured protein regions to a globular protein or by peptide-peptide complexes $^{26}$ (as shown in Figure 2), with the molecular shapes of interfaces and interactions between the binding partners being different for these classes. For example, interfaces between globular proteins are mostly flat and large and interactions at such interfaces are mainly hydrophobic interaction which typically is driven by a ring of polar residues around the interface. A mixture of hydrophobic and polar interactions and water molecules at interface is also another type of interactions at the interface between two globular proteins ${ }^{26-28}$. On the other hand, interactions between two unstructured peptides such as IDPs 
or IDPRs are predominantly driven by electrostatic interactions ${ }^{29}$, 30. Thus, identification of these PPI structural classes can be helpful to design specific types of inhibitors or stabilizers for particular PPIs. Development of compounds to modulate PPIs can be done by using different drug discovery approaches such as phenotypic screening ${ }^{31}$, target-based (or high-throughput) screening, and structure-based design ${ }^{24}$. In this review, we will focus mainly on structure-based design approaches as we have applied this method to successfully identify and develop novel inhibitors for different classes of $\mathrm{PPIs}^{32-38}$.

(a)

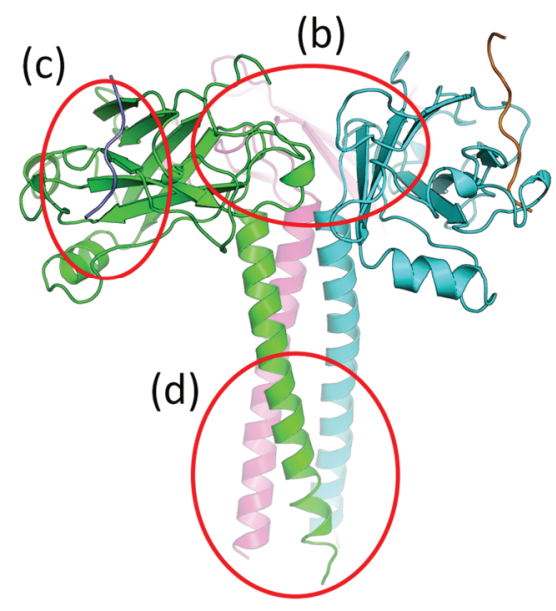

(b) globular-globular

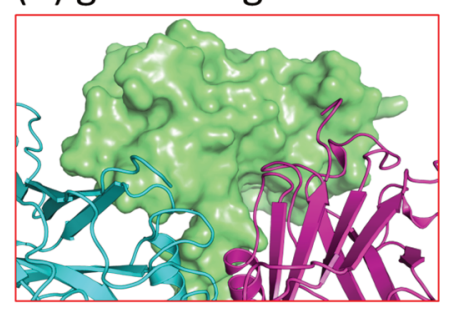

(c) globular-peptide

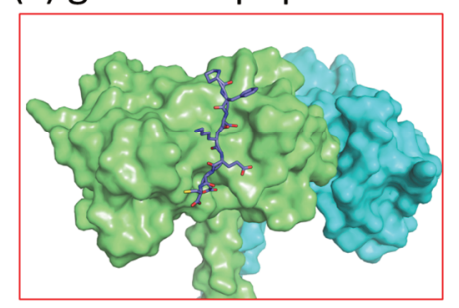

(d) peptide-peptide

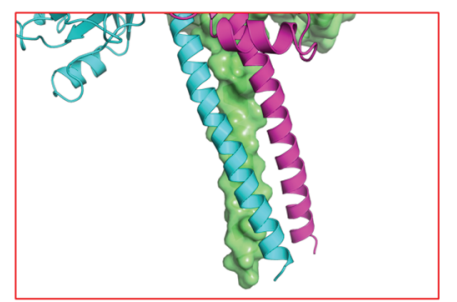

Figure 2. Different structural classes of PPIs. (A) Trimeric complex of TNF Receptor Associated Factor 2 (TRAF2) in complex with peptide (PDB code 1CA9) and molecular surface of PPI derived from different structural classes; (b) globular protein-globular protein complex, (c) globular protein with peptide, and (d) peptide with peptide (more details can be found from this review ${ }^{26}$ ).

Structure-based PPI modulator design relies on knowledge of the 3D structure of the complex of target molecules, where e.g. a protein-protein complex structure will provide information on detailed atomistic interactions between the interfaces of two proteins. This information can be utilized to rationally design small compounds, peptides, or peptidomimetics to inhibit or stabilize PPIs. Therefore, whether the aim is to develop inhibitors or stabilizers for PPIs, identification of a targeted complex is a first and crucial step towards success in structure-based molecular design for PPIs. Several methods ranging from a simple and inexpensive method such as molecular docking to the advanced technologies like Cyroelectron microscopy (Cryo-EM) ${ }^{39-41}$ can be applied for this purpose. In this review we present an overview of recent methods and technologies that are applied to identify protein-ligand or protein-protein 
complexes. This includes both experimental and predictive in silico methods Moreover, an overview will be given of computational and rational methods employed to design compounds (peptides, peptidomimetic, and small compounds) that should either inhibit or stabilize PPIs. Finally, examples will be given for the various classes of PPI modulators where novel inhibitors or stabilizers which were successfully developed by specific incorporation of 3D structures of protein-protein or proteinligand/peptide complexes providing insight into key important interactions at interface which can be used for rationally design and development of bioactive PPI modulators.

\subsection{Experimental and computational methods to identify protein-protein complexes}

\subsubsection{Experimental Methods}

Structural information of protein-protein complexes is fundamental in the rationalized design of PPI modulators which may have potential therapeutic properties. The structural information on the target of interest can be obtained from either experimental or computational methods. The determined proteinprotein complexes can assist the designer to reach a better understanding of the required and essential interactions at the atomic level of PPIs and this knowledge can then be utilized to design and develop PPI modulators. The structure of protein-protein (or protein-ligand/peptide) complexes, which can be determined by experimental methods at high-resolution, often provides such detailed information to designers, thus identifying the major contributors of the PPI to be inhibited or to be stabilized. Among different experimental methods, X-ray crystallography is a powerful technique that can be employed to solve the $3 \mathrm{D}$ structure of a protein-protein complex. However distinguishing the true protein-protein interface from artefactual crystal contacts is a significant and non-trivial challenge ${ }^{42}$. Each partner of the protein-protein complex appears to form different interfaces with the neighboring molecules in the crystal ${ }^{42}$. In addition, besides the mentioned crystal contacts, also non-physiological conditions for crystal formation may result in observation of biologically irrelevant PPIs, which can mislead the PPI modulator design ${ }^{43}$. Recently, X-ray free-electron lasers (XFEL), the use of which causes diminished radiation damage to the macromolecules of interest, are increasingly used to determine the 3D structure of protein-protein complexes. XFEL employs femtosecond X-ray pulses which allows the observation of rapid transitional changes of the structure upon ligand binding ${ }^{44}$. However, these methods have some limitations especially for using to provide structural insights for complexes which are formed between IDPs. In such cases, circular dichroism (CD) and nuclear magnetic resonance (NMR) spectroscopy are better suited as they allow the investigation of structural changes that occur upon complex formation ${ }^{30}$. NMR spectroscopy can be also used to unravel structural properties and key residues for binding of weak PPIs $\left(K_{d}>m M\right){ }^{45}$. Finally, mass spectrometry (MS) offers a great variety of different strategies to investigate the structural properties of protein-protein complexes ${ }^{46}$, such as by hydrogen-deuterium exchange MS (HDX-MS) to investigate the PPIs in protein-protein complexes ${ }^{47}$, where surface labeling is readily done with $\mathrm{D}_{2} \mathrm{O}$ based buffers ${ }^{48}$. Surface residues which are accessible to exchange, and thus not involved in complex formation, are labeled with $\mathrm{D}_{2} \mathrm{O}$ and will obtain a higher corresponding massto-charge $(\mathrm{m} / \mathrm{z})$ position over time ${ }^{48}$. A $\mathrm{m} / \mathrm{z}$ position comparison of complex and unbound proteins points out which residues are involved in complex formation ${ }^{48}$. 
The latest advances in cryo-electron microscopy (cryo-EM) allow researchers to solve the 3D structure of protein-protein complexes which have a molecular mass lower than $250 \mathrm{kDa}$ with a resolution from 3 to $5 \AA 49,50$. Cryo-EM is an increasingly popular method for structural studies of large protein complexes. Within the past years Cryo-EM has undergone rapid technological development and became capable of acquiring near-atomic level structures, which makes it suitable for use in drug discovery (besides the established methods of X-ray crystallography and NMR). Cryo-EM allows study of proteins in their nearnative (frozen-hydrated) state as well as investigation of larger and more complex systems or samples possessing multiple conformational or compositional states ${ }^{51,52}$. This may be beneficial, as inhibitorbinding pockets are often flexible in various protein conformations and prediction of hot-spots using computational techniques still remains a challenging task. The cryo-EM technique has been being continuously developed and improved and can now go beyond its earlier technical limitations. Breakthroughs came with the determination of $3 \mathrm{D}$ structures with resolutions lower than $2 \AA$ and the significant reduction in the lower molecular weight limit for samples that can be studied by cryo-EM microscopy have allowed this technique to be applied for various types of proteins e.g. membraneembedded proteins, protein-ligand complexes ${ }^{53,54}$. Currently, the cryo-EM technique has increasingly and widely been applied for molecular and structural biology study and was recently used to study PPIs such as the PRMT5 (Protein Arginine Methyl Transferase 5)-MEP50 (Methylosome Associated Protein 50) complex (molecular weight around $450 \mathrm{kDa}$ ) 55 . The obtained complex structure (resolution at $3.7 \AA$ ) shows several properties and details in agreement and consistent the previous published X-ray structures (resolutions ranging from $2.1 \AA$ to $2.9 \AA$ ) further supporting single particle cryo-EM as a high potential technique that can be applied to study human drug discovery targets ${ }^{55}$. Moreover, cryo-EM can be combined with computational methods e.g. molecular dynamics (MD) simulaitons to scrutinize molecular mechanisms and interaction networks of complex systems such as has been done for ErmBLstalled ribosomes with erythromycin (an antibiotic drug), a complex that is being studied in the context of antibiotic drug resistance. Results derived from MD simulations based on the cryo-EM structures provide insight into their fundamental mechanism which can be useful for further drug development ${ }^{56}$.

Despite the suitability of cryo-EM in molecular and structural biology studies, its application in drug discovery, either for lead identification or lead optimization has not been yet reported ${ }^{40}$. However, as supported by the promising results that are discussed above, and with the current rapid technological improvement of cryo-EM, the method is expected to be applied and considered as a powerful tool in structure-based drug design already in the very near future ${ }^{40}$. Furthermore, the combination of lowresolution experimental structures derived from cryo-EM and computational techniques like MD simulations as described above can be combined and used for the study of PPIs and for structure-based drug design.

\subsubsection{Computational Approaches}

However, obtaining atomic level information on PPIs may not always be possible due to inaccessibility to previously mentioned methods while experimental determination of protein-protein complex can in addition be laborious and costly. In such cases, use of in silico methods can help to overcome these 
limitations for predicting a likely binding mode between two proteins. Protein-protein docking is a versatile tool to study the biologically relevant interactions between two or more proteins-of-interest. Molecular docking programs such as HADDOCK ${ }^{57}$, ICM-Pro ${ }^{58}$, ZDOCK ${ }^{59}$, and $\mathrm{HDOCK}^{60}$ to name just a few can be employed to generate possible binding modes between two proteins. Molecular docking generally provides several possible docking solutions and docking solutions are then often ranked by their corresponding docking score. The top-ranked docking pose is usually selected as the most likely, native-like, structure and used for further investigation for PPIs. However, the scoring functions are built to be fast and informative, they do not entirely correlate with biochemical affinity values ${ }^{61}$ and docking scores are often not completely indicative of a most likely biologically relevant structure. Thus, selecting a likely binding pose selection based on scoring functions can be misleading61. This obstacle can be overcome by correct utilization of available biochemical knowledge of the protein-of-interest and/or good use of available structural bioinformatics methods. For example, key residues of a particular PPI derived from NMR spectroscopy ${ }^{62,63}$, or from HDX-MS data ${ }^{64}$ can be exploited in combination with docking solutions to identify a likely binding mode between two proteins. Moreover, essential residues playing important roles in PPIs of an investigated complex can also be obtained from site-directed mutagenesis experiment and the derived information can be used for assisting to build 3D computational models of protein-protein or antibody-antigen complexes ${ }^{65,66}$. Alternatively, a combination of molecular dynamics (MD) simulations and binding free energy (BFE) calculations (shown in Figure 3), which represents a more accurate approach than simple docking and scoring function, can be used together with experimental data, which can derive from different methods as mentioned above, to assist designers to distinguish false positive docking poses from native-like poses ${ }^{67,68}$. These methods can in addition reveal structural insight into such key residues of the PPIs from identified protein-protein complex and to a certain extent are able to include time-dependent considerations, like flexibility, into the process $^{34,69}$. MD simulations on these protein-protein complexes can drive the biomolecular complex to an energetically lower conformation which is more physically and biologically relevant to intermolecular interactions ${ }^{70}$. BFE calculation methods can be used to distinguish native-like docking poses from false positive docking solutions ${ }^{34}$, 68. Several methods for BFE exist, and one such method, $\mathrm{MM} / \mathrm{PB}(\mathrm{GB}) \mathrm{SA}^{71}$ (molecular mechanics/Poisson Boltzman (generalized Born) surface area) is a fast and inexpensive approach as compared to other BFE methods e.g. free energy perturbation (FEP) and thermodynamic integration (TI). A likely binding pose should be associated with the lowest BFE, implying a thermodynamically favorable conformation the pose of which stays overall stable throughout the length of an MD simulation, allowing only small RMSD fluctuations. BFE decomposition per residue (shown in Figure 3) or in silico alanine scanning are both powerful methods which can exploit the major interacting residues between binding partners. Both of these methods can indicate the type of present interaction and its contribution to the total BFE. Especially in silico alanine scanning can emphasize the effect of sterical and electrostatical changes due to an exchange of the wild-type side chain to an alanine side chain $34,63,71$. 


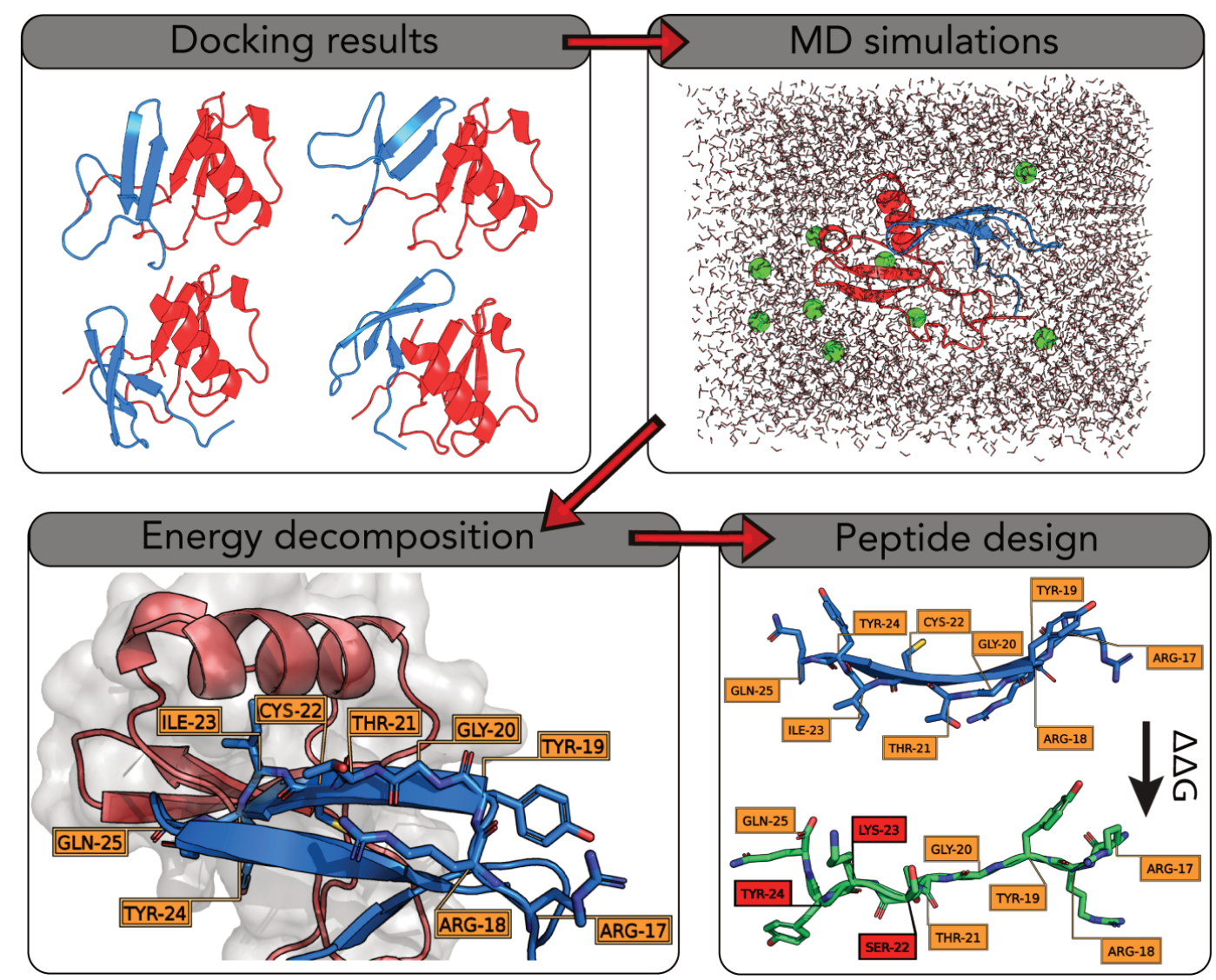

Figure 3. Schematic representation of generic approach to identify a likely binding mode of protein-protein complex served as a starting structure for inhibitor design. Protein-protein docking generates several decoys along with the likely native structure during the exploration of the PPI space. Molecular dynamics simulations can further investigate the likelihood of each docking results to form the complex-of-interest by further shifting the structures to relatively lower energy landscapes. Use of energy decomposition per residue from BFE calculations points out the PPIs at residue level and provides required information for the design of a peptide. BFE of designed peptides can be further improved by introducing single point mutation which may lower the BFE to perform better inhibition or stabilization.

In conclusion, obtaining protein-protein or protein-ligand/peptide complexes, either by computational or experimental methods can allow the identification of residues that are responsible for complex formation (shown in Figure 3). Molecular modelers can harvest such data and information i.e. key residues, secondary structure, length and composition of the protein binding partners, and approximated surface area from the determined complexes to design peptides or peptidomimetics for modulating PPIs as will be discussed in the next section.

\subsection{Peptides and Peptidomimetics}

Peptides are linear polymers of 2-50 amino acids linked together by amide bonds. In the human body many peptides such as insulin, oxytosin and cyclosporin are active $\mathrm{e}^{72-74}$ making synthetic and designer peptides attractive molecules for use in drug discovery. Furthermore, in analogy to conventional (small molecule) drugs which often mimic the substrate of an enzyme, peptides could mimic the binding epitope in PPIs, making them attractive molecules in this context ${ }^{75}$. This often makes the region of the binding partner that is involved in PPI with the target a useful starting point in the development of PPI modulators. 
Peptides extracted from such binding regions may further be subjected to in silico or in vitro alanine scanning ${ }^{76}$. This technique involves sequentially changing each residue in a peptide (via synthesis) or in a protein (via site-directed mutagenesis) to alanine. This technique is useful in identifying "hot-spot" residues, which contribute most to protein-peptide complex formation ${ }^{77}$.

Peptides however have several disadvantages, mainly due to their poor bioavailability. Peptides are often large and charged and therefore poorly or not at all absorbed following oral application. Once in the bloodstream, they are rapidly degraded by peptidases and are rapidly cleared from the body ${ }^{78}$. Furthermore, the large flexibility of peptides implies that there is an entropy penalty upon folding and binding. However, if a certain peptide has unwanted properties for a given application, it can still function as a starting point for the development of peptidomimetics. These are molecules that mimic the structure and function of a certain peptide, but do not have their inherent disadvantages ${ }^{79}$. Peptidomimetics are historically divided into three classes ${ }^{80}$. Type I are the backbone mimetics. Type II peptidomimetics are the functional mimetics, which are non-peptidic molecules that have a similar function to the parent peptide, but are not structurally related. Type III are the topological mimetics, which are molecules with a non-peptidic scaffold, but which make the same interactions.

\subsubsection{In silico techniques for the discovery and design of therapeutic peptides}

Many steps for identifying and optimizing therapeutic peptides or peptidomimimetics are amenable to in silico techniques. Like with small molecules, peptide-based drug discovery can be divided to ligand- and target-based approaches. Ligand-based techniques include multiple sequence alignments of known binding peptides or PPI epitopes to derive information about interacting amino acids. Generation and scoring of peptide conformations into a binding pocket of target proteins can be done using MD simulations or protein-peptide docking tools ${ }^{81}$.

Target based approaches can generally be divided into three stages: binding site detection, docking and affinity prediction. In the context of PPIs, the binding site is often determined by the binding interface between two proteins which can be identified by use of various experimental or computational methods as discussed in the previous section. In addition, several databases of protein-peptide interactions or several online tools can be used to search for binding hotspots between protein and peptides (more details can be found in this review ${ }^{81}$ ). The second step involves finding a likely binding pose for the peptide onto the protein. Peptide-protein docking methods can be used for this purpose. These methods can be divided in three classes, namely template-based methods, local and global docking methods ${ }^{82}$. In template-based docking a known complex is provided to the docking program. Based on this template the program attempts to find a likely binding mode for the complex under investigation ${ }^{82}$. In local docking, the user specifies the binding site and the program attempts to dock a peptide at the specified location on the surface of a target structure. Various specialized programs for protein-peptide (or protein) docking exist for this objective as described above, and also small-molecule molecular docking programs, for example AutoDock Vina ${ }^{83}$, Gold ${ }^{84}$, Glide ${ }^{85}$ and Surflex-Dock ${ }^{86}$ have been used for this purpose. While these programs have some success for peptides of up to 3 amino-acids, they are not suitable for longer chains $^{82}$. In global docking no information about the binding site is provided a priori. Thus, the docking 
programs use different algorithm to investigate suitable binding spots and consequently attempt to dock peptides onto those identified binding spots ${ }^{82}$. There are two approaches for affinity prediction: sequence based and structure-based prediction. In sequence-based affinity prediction, the amino-acids are converted to abstract amino acid property descriptors after which machine learning is used to estimate activity ${ }^{87}$. In the structural approach, the 3D structure of the protein-peptide complex is used. Various techniques are used to estimate binding affinity, such as combined quantum mechanics/molecular mechanics (QM/MM), semi-empirical Poisson-Boltzmann/surface area (PB/SA), and empirical conformational free energy analysis (CFEA $)^{88}$. Although, these methods can be time consuming, which may limit their use in screening applications, these methods can be applied in subsequent optimization steps to predict binding affinity of optimized or designed peptidic compounds. Several research groups including ours have successfully applied BFE such as MM/PBSA or MM/GBSA to estimate binding affinity of designed peptides which can significantly reduce the amount of peptides that will be synthesized and tested experimentally $34,89-91$.

\subsubsection{From peptides to peptidomimetics}

Once a functional peptide has been discovered that has the capacity to modulate a given PPI, such a peptide may provide a structural basis for the design of a peptidomimetic. Use of peptidomimetics can then offer the advantages of the peptide modulator but it has less of the known disadvantages that were discussed above for use of peptides. Many strategies exist for deriving peptidomimetics from peptides, and an example of this is illustrated in Figure 4 and Figure 5. Type I peptidomimetics can be derived by gradually changing a bio-active peptide.

The least drastic change to the original peptide is the inclusion of non-natural amino acids ${ }^{92}$ into the peptide sequence. Slightly more invasive is the cyclization of the linear peptide, which makes the peptide more rigid and thereby often less prone to degradation ${ }^{93-95}$. One strategy for cyclization is the linking of two amino acids ${ }^{75}, 96-98$. This method can be used to induce secondary structure and to provide more rigidity for the peptide. The prime example of this approach is stapling of peptides to stabilize their alphahelical form (see Figure 5 in section 4.1.1) ) $^{99}$. The introduction of rigidity or reduction in flexibility can have a positive effect on the thermodynamics (i.e. entropy) of binding of the ligand to its binding partner. Retro-inverso peptides refer to the use of D-amino acids instead of the naturally used L-amino acids. The sequence of the original peptide is then reversed to orient the residues in the same way as for the L-amino acid peptide. Retro-inversion often improves stability, since proteases are less prone to recognize the D-amino acids ${ }^{100}$. Peptoids refer to peptidomimetics in which the amino acid side chains are attached to the amide nitrogen instead of the alpha carbon ${ }^{101,102}$. In peptoids, the sequence is also reversed to keep the residues in the same orientation with respect to the backbone carbonyls. Peptoids have no backbone stereo centers and are more flexible than peptides. Finally, small molecules might be identified that capture the pharmacophoric and functional features of a parent peptide. 

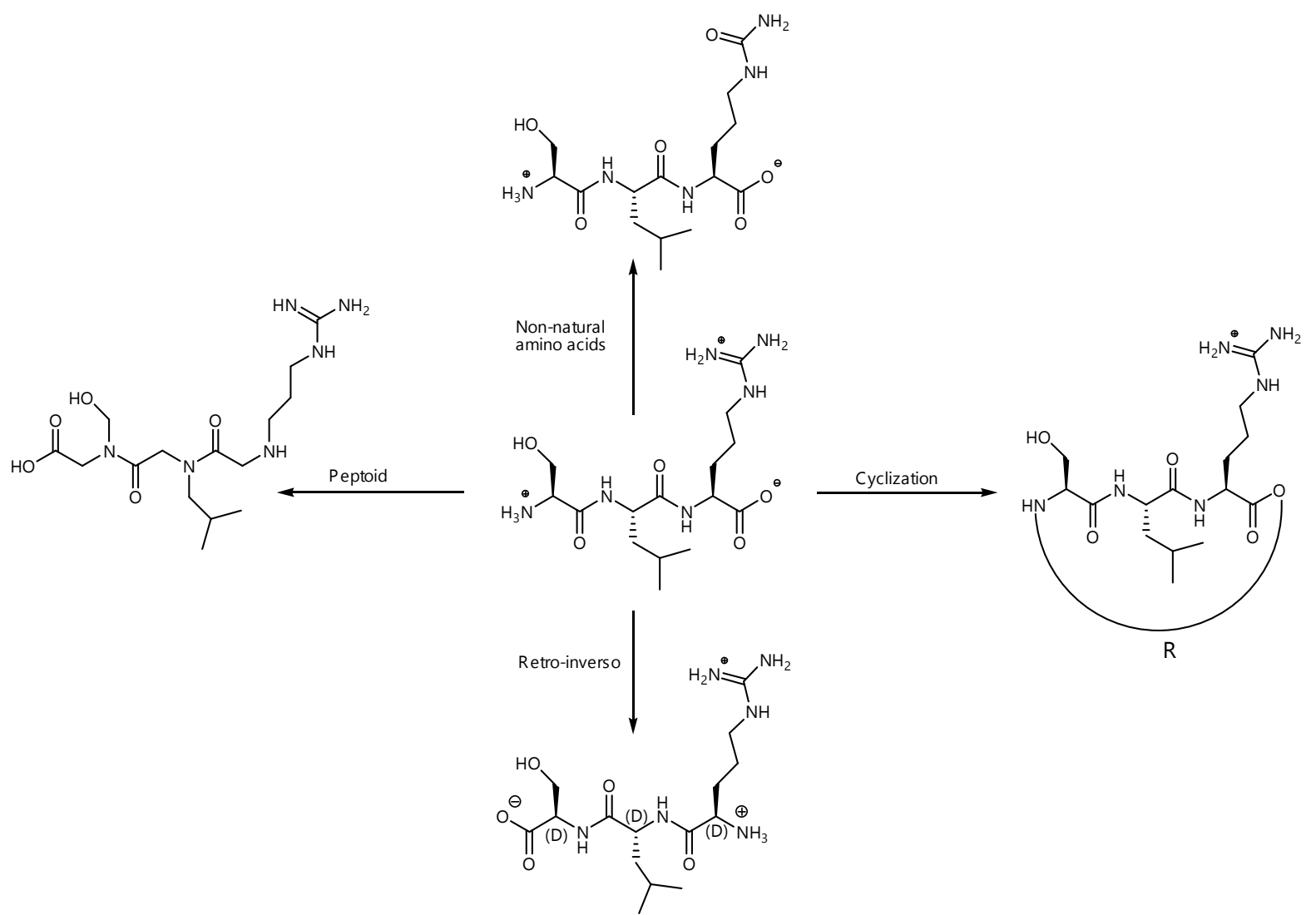

Figure 4. Examples of strategies to converse natural amino acids into peptidomimetics

In addition to the rational design and modification of peptides into stable peptidomimetic as discussed above, various in silico strategies exist for the design and discovery of peptidomimetics. These techniques are especially useful for the discovery of type II and type III peptidomimetics. De novo approaches use a non-peptide template for fitting fragments into the binding site of the target protein and then attempt to connect these fragments together ${ }^{103}$. In a sequence based approach, features of small molecules are mapped onto amino acids, allowing them to be screened against a peptide target ${ }^{104}$. Pharmacophoric approaches, either peptide-based or receptor-based, can also be employed to search for novel peptidomimetics ${ }^{104}$. In the receptor-based approach, pharmacophore features are generated based on the target structure, whereas peptide structures or any available peptide-protein complexes may be utilized to generate pharmacophore models for the peptide-based approach. A last example of in silico methods used for the design and development of peptidomimetics are geometry based approaches which attempt to discover molecules that have similar geometric features as the parent peptide ${ }^{104}$. In addition, a combination of the pharmacophoric and geometric methods can also be used to screen for peptidomimetics ${ }^{105}$ 
(a) Peptide Design
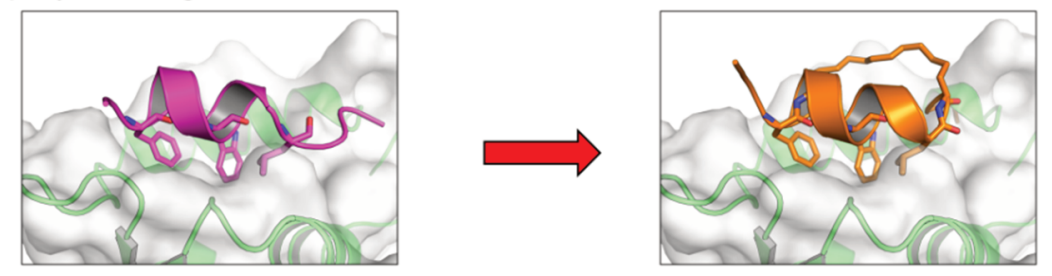

Stapled Peptide

(b) Small Compound Design

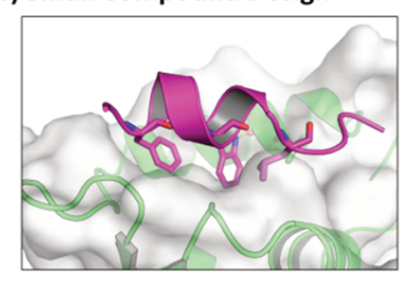

MDM2-p53 complex

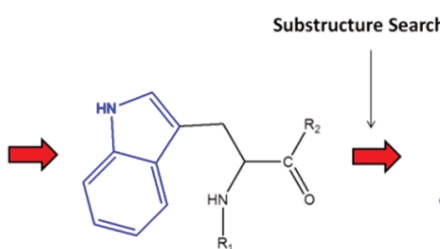

Indole ring of Trp

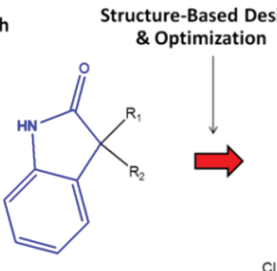

Oxindole Core Structure

\section{Stapled Peptide}

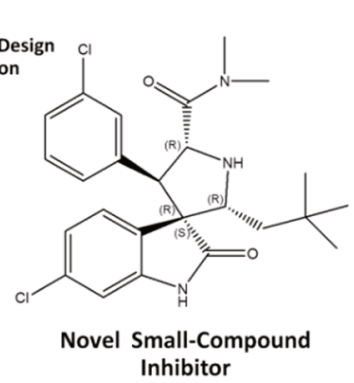

Figure 5. The 3D structure of MDM2-p53 complex (PDB Code 1YCR) can be utilized to for (a) peptide design or (b) small-compound inhibitor design. MDM2 is shown as green cartoon, molecular surface of MDM2 is displayed as white and $\alpha$-helix of p53 is illustrated in magenta. (a) The $\alpha$-helix of p53 is used as a template to build a stapled peptide (orange cartoon). (b) Indole ring of Trp from p53, which is a key residue for binding with MDM2, was applied for substructure search and the oxindole core structure was identified and subsequently used for structure-based design and optimization. By doing this way, novel small-compound inhibitors for MDM2 were developed.

\subsection{From protein-protein/peptide complex 3D structures to novel modulators of PPIs}

The drug discovery and development process is a time consuming process that generally requires a high financial investment. Rapid in silico methods like molecular docking or combinations of short MD simulations with BFE calculations (which require less computational time and cost) can however significantly accelerate the drug design process and reduce the cost of the overall drug discovery. These approaches can be applied at different steps e.g. in the identification of a 3D structure of a proteinprotein complex step and/or for design of modulators (inhibitors or stabilizers) for PPIs. Here, we present examples where the availability of 3D structures of protein-protein/peptide complexes and the in silico methods play a significant role in the development of modulators for PPIs. Furthermore, we will discuss selected cases where co-crystallization of protein-protein complexes was shown to be difficult and CryoEM was applied as an alternative to determine protein structures and protein-protein complex structures, for use in structure-based molecular design.

\subsubsection{Targeting Globular Proteins}

\subsubsection{PPI inhibitors: iPPI's}

Cancer therapy Tumor suppressor p53 plays a crucial role in protection from tumor development. During carcinogenesis, p53 is negatively regulated by MDM2 overexpression which results in a loss of its function ${ }^{106}$. The X-ray structure of the p53-MDM2 complex (PDB code 1YCR) reveals that p53-MDM2 interactions are mediated by a 15-residue $\alpha$-helical domain of p53 that includes three amino acid residues (Phe19, Trp23, and Leu26) on p53 that are critical for MDM2 binding ${ }^{107}$. This knowledge allows for the design of inhibitors to interrupt p53-MDM2 interactions, which became an attractive approach in 
the development of cancer treatments based on reactivation of p53. Although most of the p53-MDM2 inhibitor design strategies utilize alternative approaches ${ }^{108}$, simple peptidomimetics and peptide-derived compounds are recently becoming popular. For example, the $\alpha$-helical transactivation domain of p53 was used to provide a starting peptide from which optimized peptides could be derived. Peptidic inhibitors should retain its $\alpha$-helical structure and preserve the key residues for interaction with MDM2 (as displayed in Figure 5). Therefore, the concept of "peptide stapling", is applied to this $\alpha$-helical stretch of p53 by linking side chain of two non-natural amino acid residues together in order to keep a-helical conformation of the peptide as shown in Figure 5. By doing this, stabilized alpha-helix of p53 (SAH-p53) peptides were designed and optimized by stapling the p5314-29 sequence at different positions. The most potent peptide exhibits high binding affinity with an improved cellular permeability property that is better than that of the unmodified p53 peptide and also exhibits a dose-dependent inhibition of osteosarcoma cell (SJSA-1) viability $\left(\mathrm{EC}_{50}=8.8 \mu \mathrm{M}\right)^{99}$. Moreover, the structure of the $\alpha$-helix of $\mathrm{p} 53$ can also be utilized to identify small compounds to interrupt p53-MDM2 interactions (as shown in Figure 5). For instance, Ding et al ${ }^{109}$ have searched for chemical moieties that can mimic the indole ring, a side chain of Trp23 which is a key residue for interacting with MDM2. The oxindole ring which can perfectly mimic the side chain of Trp was identified and applied for substructure search for compounds containing the oxindole ring. Subsequent molecular docking was employed to investigate the binding between the identified compounds and also to assist in the modification and optimization of small-molecule inhibitors of the p53-MDM2 complex. By doing so, a potent small-molecule inhibitor was developed that can inhibit the interaction with an associated $K_{\mathrm{i}}$ value of $86 \mathrm{nM}$. These studies are excellent examples to demonstrate that protein-protein complexes and structure-based approaches can be exploited to design and develop highly potent inhibitors (either peptides or small compounds) to interrupt PPIs.

Another example of a protein family widely studied in the context of PPI modulator development is the Bcl-2 protein family which has important roles in regulation of programmed cell death. Also here represents a good example for which the novel PPI modulators are designed and developed based on available 3D structures of protein-protein complexes 118,119 . The Bcl-2 family consists of pro-apoptotic and anti-apoptotic proteins that interact with each other to form a heterodimer. Anti-apoptotic proteins of the Bcl-2 family, which are Bcl-2, Bcl-X $, \mathrm{Mcl}-1, \mathrm{Bcl}-\mathrm{B}, \mathrm{Bcl}-\mathrm{w}$ and A1/Bfl1, are overexpressed and often altered in tumor cells. Thus these proteins represent potential targets for cancer therapy. The hydrophobic groove of these anti-apoptotic proteins binds to the $\mathrm{BH} 3$ domain consisting of a 15-amino acid conserved sequence ${ }^{120}$. This information and the availability of BH3 domains-anti-apoptotic protein complexes are a good starting point and can be used for designing small compounds or peptides mimicking $\mathrm{BH} 3$ domain based on interactions between the $\mathrm{BH} 3$ domain and anti-apoptotic proteins. Some of these newly developed molecules have now entered clinical trials as shown in Table 1. 
Table 1. Example of small compounds or peptides targeting different protein targets in the Bcl-2 protein family. These inhibitors were identified and developed by using different approaches e.g. structure-based design by mimicking interactions between the BH3 domain and anti-apoptotic proteins or high-throughput screening method.

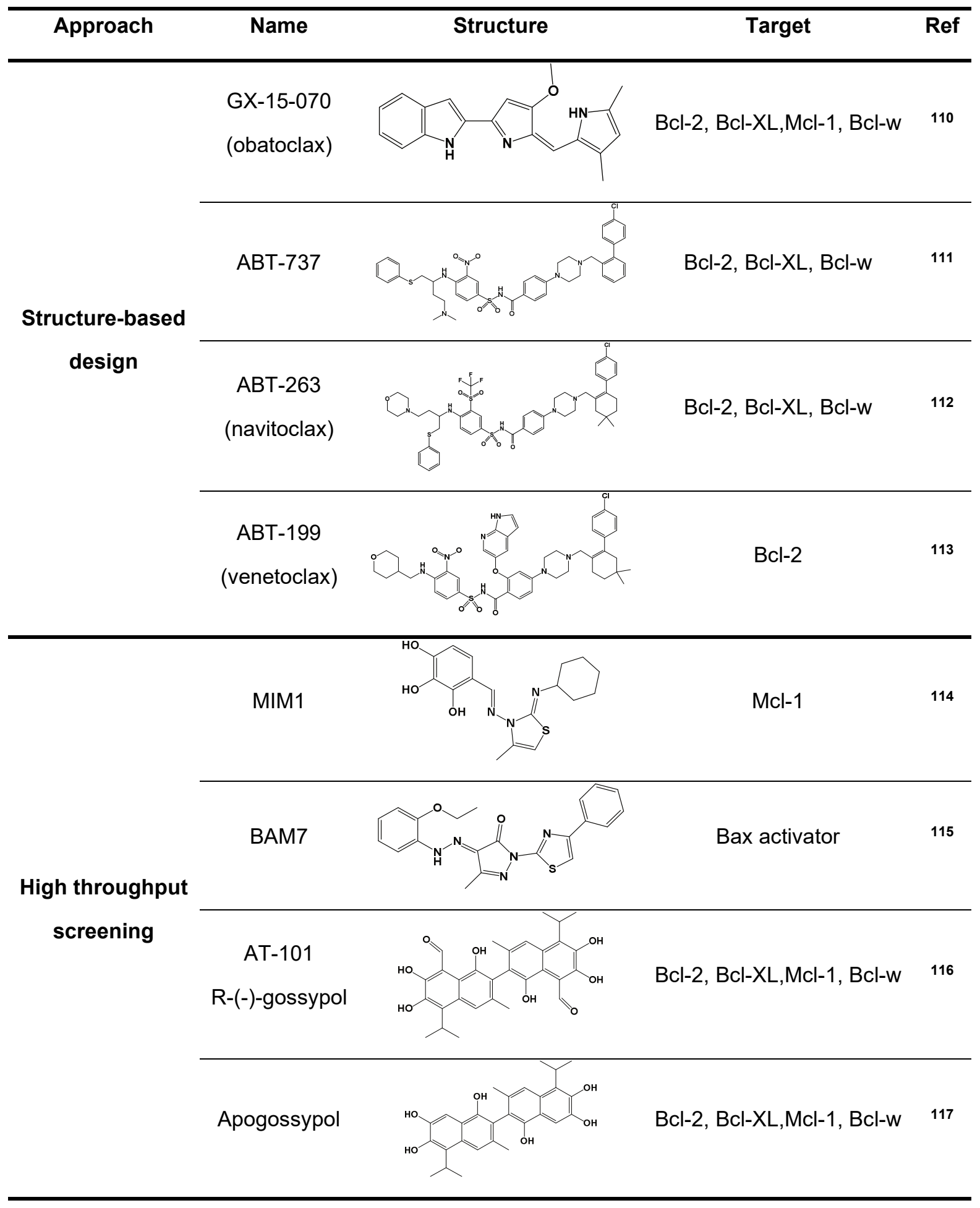

Therapy of inflammatory disorders Inflammatory responses are regulated by different pathways such as via interactions between chemokines and their specific receptors e.g CXCL8-CXCR1 interactions ${ }^{89}$ or through a recently discovered mechanism via ligation of a CCL5-HNP1 heterodimeric complex with 
CCR5 ${ }^{35}$. These two cases are excellent examples where in silico methods can play important roles for the study of PPIs and the possible translation of the structural knowledge to design peptidic inhibitors. To experimentally determine the 3D structure of a CXCL8-CXCR1 complex either by X-ray crystallography or CryoEM is laborious, and at present no X-ray complex structures of the CCL5-HNP1 complex are available in the Protein Data Bank ${ }^{121}$. Therefore, alternative fast and inexpensive in silico approaches (i.e. combination of molecular docking, MD simulations, and BFE calculations) were utilized to identify a likely binding mode of the CXCL8-CXCR189, 122 and of the CCL5-HNP1 complex ${ }^{34}$. Subsequently, the identified complexes were exploited to design peptides that can interrupt CXCL8CXCR1 and CCL5-HNP1 interactions. For example, from the derived CXCL8-CXCR1 complex several peptides were designed based on the N-loop of CXCL8 which is the main part that interacts with the $\mathrm{N}$ terminus of CXCR1. The most potent peptidic inhibitor binds to the CXCR1 derived peptide exhibiting $\mathrm{K}_{\mathrm{D}}$ at micromolar level $(252 \mu \mathrm{M})$ and this derived peptide also shows additionally activity in vivo by inhibiting monocytes adhesion transmigration ${ }^{89}$. Likewise, a series of peptides based on the HNP1 sequence that bind to CCL5 was rationally designed and the binding affinities of individual peptides to CCL5 was in silico predicted ${ }^{34}$. By doing this way, only 10 peptides from more than a billion possible candidate peptides were selected and synthesized and in vitro tested. The most potent inhibitor called "SKY peptide" was chosen and then tested its inhibitory activity in vivo and a mouse model of myocardial infarction and results reveal that inhibition of CCL5-HNP1 interactions by "SKY" peptide can inhibit monocyte adhesion and reduce myocardial macrophage accumulation. ${ }^{35}$. These studies demonstrate that in silico methods can be efficiently used to determine protein-protein complexes and rationally design inhibitors that have a potential for further development as anti-inflammation drugs. Furthermore, a combination of NMR study, MD simulations and BFE calculations can be utilized to identify a heterodimeric complex of different chemokine pairs e.g. CCL5-CXCL4, CCL5-CCL17, and CCL5CXCL12 heterodimer. The derived complexes provide structural information such as on the type of interactions. Also these complexes can be analysed via computational methods to identify key interacting residues at the interface that can be exploited to design stable and specific peptidic inhibitors to prevent complex formation of chemokine heterodimeric pairs. These inhibitory peptides are active not only in vitro, they also show inhibitory activity in different animal models of inflammatory diseases 33,123 .

\subsubsection{PPI Stabilizers: sPPI's}

Some of the most extensively studied proteins for the development of PPI stabilizers are the 14-3-3 proteins which are a family of conserved regulatory molecules that can bind to different partners and are involved in many cellular functions such as apoptosis, cell cycle regulation, and signal transduction ${ }^{124}$. Fortunately, X-ray structures of the 14-3-3 proteins in complex with different binding partners are available from the Protein Data Bank allowing molecular modelers to use these structures for rational design of selective stabilizers for different protein-protein complexes 22, 125, 126. For instance, recently, MD simulations were applied to investigate the interaction networks of human 14-3-3 sigma in complex with TASK-3 peptide and the stabilizer Fusicoccin A (PDB code 3P10). The derived results from the MD simulations provide insight into key interactions of the complex which were then utilized to rationally design PPI stabilizers. As suggested by MD simulations, the novel PPI stabilizers which show 
additional interactions with the identified key residues exhibit significantly improved potency ${ }^{127}$. This study clearly demonstrates the robustness of the combination of 3D structures and in silico approaches for the rational design of PPI modulators.

Human calcium-activated $\mathrm{K}+$ channels $(\mathrm{KCa})$ of the small- $(\mathrm{SK})$ and intermediate-conductance (IK) $\mathrm{K}+$ channel types are reported to be involved in several diseases, such as cancer, autoimmune disorders, neurodegenerative disease and vascular inflammation ${ }^{128}$. Similar to the work on 14-3-3- mentioned above, the availability of $\mathrm{X}$-ray structures of SK2 (calcium-activated $\mathrm{K}+$ channel 2 ) with $\mathrm{Ca}^{2+}$-bound calmodulin (CaM) and the stabilizers (1-EBIO and PHU) were applied for the development of novel stabilizers (NS309, CyPPA, and DCEBIO). Due to the poor solubility of NS309 and CyPPA and the difficulty to co-crystallize a complex with these compounds bound to it, researchers were inspired to perform molecular docking and MD simulations instead to examine the binding of these compounds with the SK2-CaM complexes ${ }^{129}$. The derived results were utilized for further development of stabilizers with improved binding affinity and selectivity. This shows that in such cases where co-crystallization of protein complexes has some limitations, in silico methods can provide an alternative for rational modulator design and protein-protein-ligand interaction study.

\subsubsection{Targeting Intrinsically Disordered Proteins (IDPs) / Regions (IDPRs) to interrupt PPIs}

A major challenge in targeting IDPs and IDPRs are the limitations of experimental and computational methods used to completely characterize the heterogeneous conformations and to identify transient druggable pockets or binding sites of IDPs/IDPRs and how to exploit these structures through drug discovery ${ }^{130}$. However, computational methods such as different enhancing MD simulations techniques or coarse-grained simulations can be used to investigate dynamic motions, flexibility, secondary structures, and transient pockets of IDPs/IDPRs ${ }^{131-135}$. One of the most studied disordered PPIs and promising cancer drug targets is the C-MYC:MAX complex ${ }^{136-139}$. In their monomer conformation, both C-MYC and MAX are IDPs but upon complex formation they fold into a secondary structure to form a stable heterodimer. Two different strategies can be utilized to develop inhibitors for the disruption of cMYC:MAX interactions (as displayed in Figure 6). The first approach is called "TRAP mode" in which the unfolded C-MYC conformation is used as a target to search for compounds that can bind this nonfunctional conformation and that prevents it from binding with the MAX protein ${ }^{140}$ (as shown in Figure 6). Jin et al. ${ }^{15}$ and Yu et al. ${ }^{16}$ have applied replica exchange molecular dynamics (REMD) simulations to examine the folding and conformational changes of the c-Myc $370-409$ peptide and have used different conformations extracted from REMD for virtual screening. They have identified novel small compounds binding the C-Myc $370-409$ peptide that show inhibitory a ctivity at the micromolar level in in vitro assays that also inhibiting the growth of c-Myc-overexpressing cells ${ }^{16}$. Another strategy to develop modulators to interrupt c-MYC:MAX interactions is called "COMPETITIVE mode" and with this strategy the stable dimeric c-MYC:MAX complex is utilized to rationally design compounds to inhibit c-MYC:MAX interactions (as also shown in Figure 6). Since experimental 3D structures of C-MYC:MAX complexes are available from the PDB, this approach has more advantages and it is theoretically easier since the inhibitor design is based on the properties and interactions of the complex.Different types of inhibitors, 
for instance stapled MAX-derived peptides, peptidomimetics, a mutant of the C-terminal region of MYC (termed OMOMYC) ${ }^{142,143}$ are developed and designed based on the C-MYC:MAX complex ${ }^{140,}{ }^{144}$. Several clinically relevant disordered proteins such as A $\beta$, EWS-Fli1, STAT3, HIF-1, and $\beta$-catenin are currently being targeted for development of new drugs and extensive computational methods can be applied to investigate binding pocket and protein conformations or to screen for novel inhibitors ${ }^{140,} 145-147$.

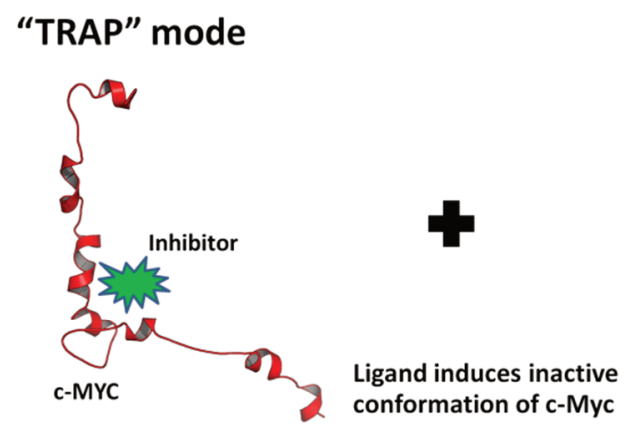

\section{"COMPETITIVE" mode}
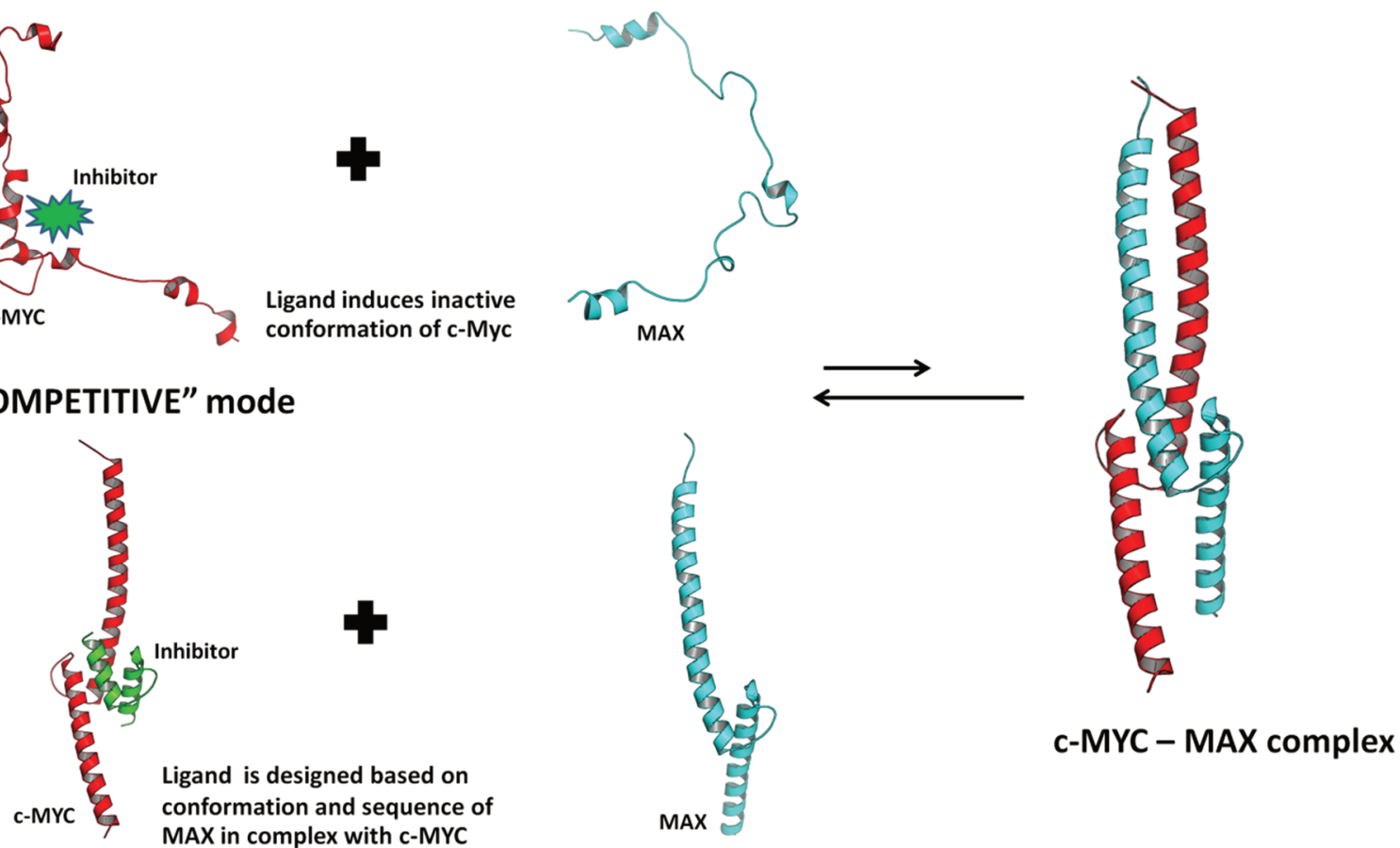

Figure 6. Different binding mode and binding mechanism of inhibitors that can prevent C-MYC-MAX complex formation ${ }^{140,141}$

\subsection{Conclusion}

During the last 20 years there has been a rapid growth in the amounts of modulators (inhibitors or stabilizers) that able to target PPIs. This development is a clear indication that PPIs are claiming their position in drug discovery and development research. The realization that such flat surfaces at the PPI interface and unstructured parts of IDPs/IDPRs can be targeted by peptides or small compounds has sparked increasing interests by drug researchers and modelers to explore these structures, which used to be considered as not druggable. Certainly, currently prescribed drugs are not limited to only small compounds. Several peptides have already been approved by the responsible authorities or are now entering the clinical stages of development ${ }^{74,78}$. Furthermore, peptides can be utilized as a starting structure to develop peptidomimetics or small compounds with improved solubility and cell permeability properties. Therefore, modulations of PPIs by peptides represent a trend that receives rapidly increasing interest from the drug discovery research field. Targeting the unstructured parts of IDPs/IDPRs which are involved in PPIs is now becoming another trend in the research field of drug discovery and 
development. This trend if fueled by smart use of several technologies e.g. CD- or NMR-spectroscopy or in silico methods such as MD simulations that can be applied to investigate protein secondary structure and presence of transient binding pockets for the IDPs/IDPRs studied. These additional data can be utilized for development of inhibitors.

Several studies as discussed here have shown that the identified protein-protein complexes either from experimental or computational approaches can be used to design inhibitors or stabilizers to modulate PPIs. Moreover, several PPI modulators are now entering the clinical stages. Therefore, these are clear evidences to prove that PPIs represent promising drug targets and protein-protein complexes can be useful for using as a starting structure to design peptide, peptidomimetic or small compounds to modulate PPIs.

\subsection{Future Perspectives}

Determination of 3D structures of target protein-protein complexes is the key step to success in structure-based molecular design for PPIs. Experimental methods (e.g. X-ray crystallography and NMR spectroscopy) and computational methods (e.g. molecular docking and MD simulations) can be used for this purpose. The high performance of computers and MD simulation packages nowadays enable us to perform simulations on specialized processors, GPUs, which can significantly speed up and accelerate calculations ${ }^{148,149}$. Therefore, MD simulations can now be routinely employed to investigate protein dynamic motions, conformational changes, and detailed atomistic interactions at the interface of protein-protein complexes and also to identify the presence of transient druggable pockets in IDPs/IDPRs. This derived information can be exploited to rationally design PPI modulators. Moreover, fast and accurate BFE methods have been applied to predict binding affinity of compounds and several studies have demonstrated that this approach can be applied for this purpose. Thus, BFE methods can be used to (relatively) estimate binding affinities for prioritizing compounds prior to their synthesis and experimental testing of their biological activity. Taken together, computational methods represent a powerful tool that can be applied together with experimental methods for the study of PPIs and for molecular design applications. These in silico methods are now increasingly used in medicinal and pharmaceutical chemistry research to guide and support experimentalists.

Cryo-EM technology is currently rapidly emerging in the biomedical research field and it has been applied to study the molecular structure of different protein classes. Even though the application of this method in structure-based drug design has not yet been well described and widely used, the groundbreaking determination of 3D structures of low-molecular-weight therapeutic proteins with high resolutions by means of cryo-EM technique is indicative of the clear promise this technique holds for application in structure-based molecular design in the near future. Not only high resolution cryo-EM structures, also low resolutions cryo-EM structures can serve as a basis to perform extensive MD simulations, so as to study structural and dynamic properties of proteins and to investigate PPIs. For such cases in which determination of 3D structures of complexes either by X-ray crystallography or cryoEM technique is difficult to achieve, NMR or mass spectroscopy can also alternatively be employed to 
scrutinize key residues for PPIs. The derived results can be combined with in silico approaches to determine 3D complexes as described above.

\subsection{Executive summary}

\subsubsection{Protein-protein complexes as drug targets}

The horizon of drug discovery is now expanding in the direction of PPIs, while vast amounts of PPIs are still not yet characterized and explored. Moreover, a vast body of experimental results has evidently proven that different classes of PPIs, including those of difficult targets such as IDPs/IDPRs, can be targeted and thus represent promising drug targets for current and future drug discovery research.

\subsubsection{Identification of protein-protein complexes}

A high resolution and quality of 3D structures of protein-protein/peptide complexes can be obtained by applications of experimental techniques such as NMR spectroscopy and X-ray crystallography and the determined 3D complexes can be exploited for structure-based drug design. Recent advancements in cryo-EM technology has caused a considerable increased interested in this technology in the molecular and structural biology field, where it is able to determine 3D structures of proteins, while still having several limitations for application in drug design. However, with the rapid technical improvement for cryo-EM, this method is expected to be applied as a powerful tool for drug discovery and design soon in the near future.

As an alternative to experimental methods, fast and inexpensive in silico methods such as molecular docking and MD simulations can be applied to identify and determine 3D structures of protein-protein complexes. These methods, especially enhancing sampling techniques in MD simulations, have more advantages than the experimental methods for the investigation and exploration of the energy landscape and conformational space of IDPs/IDPRs.

\subsubsection{Modulations of PPIs by peptides or peptidomimetics}

3D structures of protein-protein/peptide complexes, either derived by experimental or computational approaches, can be exploited to rationally design inhibitors or stabilizers to modulate PPIs. Parts of protein (or peptide) structure derived from a binding partner like a. beta-strand, loop, helix or even just one single key interacting amino acid residue can be utilized as a starting point for the development of novel and potent peptides, peptidomimetics or small compounds to modulate PPIs.

\section{$2.8 \quad$ Acknowledgement}

This work has received funding from the Horizon 2020 Framework Program for Research and Innovation of the European Union under Grant Agreement No 675746 (J.H.; B.E.; G.A.F.N.) and from strategic project funding by the Amsterdam University Medical Centre (to H.P., Grant No. 681483). X.L. has received funding from the China Scholarship Council. 


\section{References}

1. Braun, P.; Gingras, A. C., History of protein-protein interactions: From egg-white to complex networks. Proteomics 2012, 12 (10), 1478-1498.

2. Petta, I., et al., Modulation of Protein-Protein Interactions for the Development of Novel Therapeutics. Mol. Ther. 2016, 24 (4), 707-18.

3. Ryan, D. P.; Matthews, J. M., Protein-protein interactions in human disease. Curr. Opin. Struct. Biol. 2005, 15 (4), 441-6.

4. Bosch, J., PPI inhibitor and stabilizer development in human diseases. Drug Discov. Today Technol. 2017, 24, 3-9.

5. Ercig, B., et al., Insights into 3D Structure of ADAMTS13: A Stepping Stone towards Novel Therapeutic Treatment of Thrombotic Thrombocytopenic Purpura. Thromb. Haemost. 2018, 118 (1), 28-41.

6. Villoutreix, B. O.; Miteva, M. A., Discoidin Domains as Emerging Therapeutic Targets. Trends Pharmacol. Sci. 2016, 37 (8), 641-659.

7. Arkin, M. R., et al., Small-molecule inhibitors of protein-protein interactions: progressing toward the reality. Chem. Biol. 2014, 21 (9), 1102-14.

8. Fuller, J. C., et al., Predicting druggable binding sites at the protein-protein interface. Drug Discov. Today 2009, 14 (3-4), 155-161.

9. Rognan, D., Rational design of protein-protein interaction inhibitors. MedChemComm 2015, 6 (1), 51-60.

10. Arkin, M. R.; Wells, J. A., Small-molecule inhibitors of protein-protein interactions: progressing towards the dream. Nat. Rev. Drug Discov. 2004, 3 (4), 301-17.

11. Labbe, C. M., et al., iPPI-DB: an online database of modulators of protein-protein interactions. Nucleic Acids Res. 2016, 44 (D1), D542-7.

12. Wojcik, P.; Berlicki, L., Peptide-based inhibitors of protein-protein interactions. Bioorg. Med. Chem. Lett. 2016, 26 (3), 707-713.

13. Kuenemann, M. A., et al., In silico design of low molecular weight protein-protein interaction inhibitors: Overall concept and recent advances. Prog. Biophys. Mol. Biol. 2015, 119 (1), 20-32.

14. Stumpf, M. P., et al., Estimating the size of the human interactome. Proc. Natl. Acad. Sci. U. S. A. 2008, 105 (19), 6959-64.

15. Jin, F., et al., Ligand clouds around protein clouds: a scenario of ligand binding with intrinsically disordered proteins. PLoS Comput. Biol. 2013, 9 (10), e1003249.

16. Yu, C., et al., Structure-based Inhibitor Design for the Intrinsically Disordered Protein c-Myc. Sci. Rep. 2016, 6, 22298.

17. Kukar, T. L., et al., Substrate-targeting gamma-secretase modulators. Nature 2008, 453 (7197), 925-9.

18. Wildhagen, K. C., et al., Nonanticoagulant heparin prevents histone-mediated cytotoxicity in vitro and improves survival in sepsis. Blood 2014, 123 (7), 1098-101.

19. Krishnan, N., et al., Targeting the disordered $\mathrm{C}$ terminus of PTP1B with an allosteric inhibitor. Nat Chem Biol 2014, 10 (7), 558-66.

20. Lantz, K. A., et al., Inhibition of PTP1B by trodusquemine (MSI-1436) causes fat-specific weight loss in diet-induced obese mice. Obesity (Silver Spring) 2010, 18 (8), 1516-23.

21. Milroy, L. G., et al., Stabilizer-Guided Inhibition of Protein-Protein Interactions. Angew Chem Int Ed Engl 2015, 54 (52), 15720-4.

22. Zarzycka, B., et al., Stabilization of protein-protein interaction complexes through small molecules. Drug Discov. Today 2016, 21 (1), 48-57.

23. Fischer, G., et al., Alternative modulation of protein-protein interactions by small molecules. Curr. Opin. Biotechnol. 2015, 35, 78-85.

24. Modell, A. E., et al., Systematic Targeting of Protein-Protein Interactions. Trends. Pharmacol. Sci. 2016, 37 (8), 702-713.

25. Wang, N., et al., Dissecting allosteric effects of activator-coactivator complexes using a covalent small molecule ligand. Proc. Natl. Acad. Sci. U. S. A. 2014, 111 (33), 12061-6.

26. Scott, D. E., et al., Small molecules, big targets: drug discovery faces the protein-protein interaction challenge. Nat. Rev. Drug Discov. 2016, 15 (8), 533-50.

27. Miller, S., The Structure of Interfaces between Subunits of Dimeric and Tetrameric Proteins. Protein. Eng. 1989, 3 (2), 77-83.

28. Larsen, T. A., et al., Morphology of protein-protein interfaces. Structure 1998, 6 (4), 421-7.

29. Keskin, O., et al., Principles of protein-protein interactions: what are the preferred ways for proteins to interact? Chem. Rev. 2008, 108 (4), 1225-44.

30. Borgia, A., et al., Extreme disorder in an ultrahigh-affinity protein complex. Nature 2018, 555 (7694), 6166.

31. Moffat, J. G., et al., Phenotypic screening in cancer drug discovery - past, present and future. Nat. Rev. Drug Discov. 2014, 13 (8), 588-602. 
32. Seijkens, T. T. P., et al., Targeting CD40-Induced TRAF6 Signaling in Macrophages Reduces Atherosclerosis. J. Am. Coll. Cardiol. 2018, 71 (5), 527-542.

33. von Hundelshausen, P., et al., Chemokine interactome mapping enables tailored intervention in acute and chronic inflammation. Sci. Transl. Med. 2017, 9 (384).

34. Wichapong, K., et al., Structure-Based Design of Peptidic Inhibitors of the Interaction between CC Chemokine Ligand 5 (CCL5) and Human Neutrophil Peptides 1 (HNP1). J. Med. Chem. 2016, 59 (9), 4289-301.

35. Alard, J. E., et al., Recruitment of classical monocytes can be inhibited by disturbing heteromers of neutrophil HNP1 and platelet CCL5. Sci. Transl. Med. 2015, 7 (317), 317ra196.

36. Zarzycka, B., et al., Discovery of small molecule CD40-TRAF6 inhibitors. J. Chem. Inf. Model. 2015, 55 (2), 294-307.

37. Nicolaes, G. A., et al., Rational design of small molecules targeting the $\mathrm{C} 2$ domain of coagulation factor VIII. Blood 2014, 123 (1), 113-20.

38. Sperandio, O., et al., Identification of novel small molecule inhibitors of activated protein C. Thromb. Res. 2014, 133 (6), 1105-14.

39. Adrian, M., et al., Cryo-electron microscopy of viruses. Nature 1984, 308 (5954), 32-6.

40. Renaud, J. P., et al., Cryo-EM in drug discovery: achievements, limitations and prospects. Nat. Rev. Drug Discov. 2018, 17 (7), 471-492.

41. Afanasyev, P., et al., Single-particle cryo-EM using alignment by classification $(A B C)$ : the structure of Lumbricus terrestris haemoglobin. IUCrJ 2017, 4 (Pt 5), 678-694.

42. Kobe, B., et al., Crystallography and protein-protein interactions: biological interfaces and crystal contacts. Biochem. Soc. Trans. 2008, 36, 1438-1441.

43. Dafforn, T. R., So how do you know you have a macromolecular complex? Acta Crystallogr. Sect. D Biol. Crystallogr. 2007, 63, 17-25.

44. Lyubimov, A. Y., et al., Advances in X-ray free electron laser (XFEL) diffraction data processing applied to the crystal structure of the synaptotagmin-1 / SNARE complex. Elife 2016, 5.

45. Vaynberg, J.; Qin, J., Weak protein-protein interactions as probed by NMR spectroscopy. Trends Biotechnol. 2006, 24 (1), 22-27.

46. Fort, K. L., et al., Expanding the structural analysis capabilities on an Orbitrap-based mass spectrometer for large macromolecular complexes. Analyst 2018, 143 (1), 100-105.

47. D'Arcy, S., et al., Chaperone Nap1 Shields Histone Surfaces Used in a Nucleosome and Can Put H2AH2B in an Unconventional Tetrameric Form. Mol. Cell 2013, 51 (5), 662-677.

48. Lossl, P., et al., The diverse and expanding role of mass spectrometry in structural and molecular biology. EMBO. J. 2016, 35 (24), 2634-2657.

49. Shen, K., et al., Architecture of the human GATOR1 and GATOR1-Rag GTPases complexes. Nature 2018, 556 (7699), 64-69.

50. Bai, X. C., et al., An atomic structure of human gamma-secretase. Nature 2015, 525 (7568), 212-217.

51. Roh, S. H., et al., Subunit conformational variation within individual GroEL oligomers resolved by CryoEM. Proc. Natl. Acad. Sci. U. S. A. 2017, 114 (31), 8259-8264.

52. Frank, J., Generalized single-particle cryo-EM--a historical perspective. Microscopy (Oxf) 2016, 65 (1), 38.

53. Nogales, E., The development of cryo-EM into a mainstream structural biology technique. Nat. Methods 2016, 13 (1), 24-7.

54. Bartesaghi, A., et al., 2.2 A resolution cryo-EM structure of beta-galactosidase in complex with a cellpermeant inhibitor. Science 2015, 348 (6239), 1147-51.

55. Timm, D. E., et al., Cryo-electron microscopy structure of a human PRMT5:MEP50 complex. PLoS One 2018, 13 (3), e0193205.

56. Arenz, S., et al., A combined cryo-EM and molecular dynamics approach reveals the mechanism of ErmBL-mediated translation arrest. Nat. Commun. 2016, 7, 12026.

57. van Zundert, G. C. P., et al., The HADDOCK2.2 Web Server: User-Friendly Integrative Modeling of Biomolecular Complexes. J. Mol. Biol. 2016, 428 (4), 720-725.

58. Abagyan, R., et al., Icm - a New Method for Protein Modeling and Design - Applications to Docking and Structure Prediction from the Distorted Native Conformation. J. Comput. Chem. 1994, 15 (5), 488-506.

59. Pierce, B. G., et al., ZDOCK server: interactive docking prediction of protein-protein complexes and symmetric multimers. Bioinformatics 2014, 30 (12), 1771-3.

60. Yan, Y., et al., HDOCK: a web server for protein-protein and protein-DNA/RNA docking based on a hybrid strategy. Nucleic Acids Res. 2017, 45 (W1), W365-W373.

61. Warren, G. L., et al., A critical assessment of docking programs and scoring functions. J. Med. Chem. 2006, 49 (20), 5912-31.

62. Montalvao, R. W., et al., Structure determination of protein-protein complexes using NMR chemical shifts: case of an endonuclease colicin-immunity protein complex. J. Am. Chem. Soc. 2008, 130 (47), 15990-6. 
63. Miller, M. C., et al., Adhesion/growth-regulatory galectins tested in combination: evidence for formation of hybrids as heterodimers. Biochem. J. 2018, 475, 1003-1018.

64. Roberts, V. A., et al., Combining H/D Exchange Mass Spectrometry and Computational Docking To Derive the Structure of Protein-Protein Complexes. Biochemistry 2017, 56 (48), 6329-6342.

65. Wichapong, K., et al., Homology modeling and molecular dynamics simulations of Dengue virus NS2B/NS3 protease: insight into molecular interaction. J Mol Recognit 2010, 23 (3), 283-300.

66. Loyau, J., et al., Robust Antibody-Antigen Complexes Prediction Generated by Combining Sequence Analyses, Mutagenesis, In Vitro Evolution, X-ray Crystallography and In Silico Docking. J Mol Biol 2015, 427 (16), 2647-62.

67. Shimba, N., et al., Model Building of Antibody-Antigen Complex Structures Using GBSA Scores. J. Chem. Inf. Model. 2016, 56 (10), 2005-2012.

68. Simoes, I. C. M., et al., Properties that rank protein:protein docking poses with high accuracy. Phys. Chem. Chem. Phys. 2018, 20 (32), 20927-20942.

69. Rohe, A., et al., In vitro and in silico studies on substrate recognition and acceptance of human PKMYT1, a Cdk1 inhibitory kinase. Bioorg. Med. Chem. Lett. 2012, 22 (2), 1219-23.

70. Tovchigrechko, A.; Vakser, I. A., How common is the funnel-like energy landscape in protein-protein interactions? Protein Sci. 2001, 10 (8), 1572-1583.

71. Miller, B. R., et al., MMPBSA.py: An Efficient Program for End-State Free Energy Calculations. J. Chem. Theory Comput. 2012, 8 (9), 3314-3321.

72. Tsomaia, N., Peptide therapeutics: targeting the undruggable space. Eur. J. Med. Chem. 2015, 94, 45970.

73. Fosgerau, K.; Hoffmann, T., Peptide therapeutics: current status and future directions. Drug Discov. Today 2015, 20 (1), 122-8.

74. Lau, J. L.; Dunn, M. K., Therapeutic peptides: Historical perspectives, current development trends, and future directions. Bioorg. Med. Chem. 2018, 26 (10), 2700-2707.

75. Pelay-Gimeno, M., et al., Structure-Based Design of Inhibitors of Protein-Protein Interactions: Mimicking Peptide Binding Epitopes. Angew. Chem. Int. Ed. Engl. 2015, 54 (31), 8896-927.

76. Cunningham, B. C.; Wells, J. A., High-resolution epitope mapping of hGH-receptor interactions by alaninescanning mutagenesis. Science 1989, 244 (4908), 1081-5.

77. Clackson, T.; Wells, J. A., A hot spot of binding energy in a hormone-receptor interface. Science 1995, 267 (5196), 383-6.

78. Henninot, A., et al., The Current State of Peptide Drug Discovery: Back to the Future? J. Med. Chem. 2018, $61(4), 1382-1414$.

79. Hummel, G., et al., Translating peptides into small molecules. Mol. Biosyst. 2006, 2 (10), 499-508.

80. Ripka, A. S.; Rich, D. H., Peptidomimetic design. Curr. Opin. Chem. Biol. 1998, 2 (4), 441-52.

81. Diller, D. J., et al., Rational, computer-enabled peptide drug design: principles, methods, applications and future directions. Future Med. Chem. 2015, 7 (16), 2173-93.

82. Ciemny, M., et al., Protein-peptide docking: opportunities and challenges. Drug Discov. Today 2018, 23 (8), 1530-1537.

83. Trott, O.; Olson, A. J., AutoDock Vina: improving the speed and accuracy of docking with a new scoring function, efficient optimization, and multithreading. J. Comput. Chem. 2010, 31 (2), 455-61.

84. Jones, G., et al., Development and validation of a genetic algorithm for flexible docking. J. Mol. Biol. 1997, 267 (3), 727-48.

85. Friesner, R. A., et al., Glide: a new approach for rapid, accurate docking and scoring. 1. Method and assessment of docking accuracy. J. Med. Chem. 2004, 47 (7), 1739-49.

86. Spitzer, R.; Jain, A. N., Surflex-Dock: Docking benchmarks and real-world application. J. Comput. Aided Mol. Des. 2012, 26 (6), 687-99.

87. Zhou, P., et al., Computational Peptidology: A New and Promising Approach to Therapeutic Peptide Design. Curr. Med. Chem. 2013, 20 (15), 1985-1996.

88. Tian, F., et al., Characterization of PDZ domain-peptide interactions using an integrated protocol of QM/MM, PB/SA, and CFEA analyses. J. Comput. Aided Mol. Des. 2011, 25 (10), 947-58.

89. Jiang, S. J., et al., Peptides derived from CXCL8 based on in silico analysis inhibit CXCL8 interactions with its receptor CXCR1. Sci. Rep. 2015, 5, 18638.

90. Greene, D., et al., Computational Analysis for the Rational Design of Anti-Amyloid Beta (Abeta) Antibodies. J. Phys. Chem. B 2018, 122 (16), 4521-4536.

91. Wang, C., et al., Recent Developments and Applications of the MMPBSA Method. Front. Mol. Biosci. 2017, 4, 87.

92. Gentilucci, L., et al., Chemical modifications designed to improve peptide stability: incorporation of nonnatural amino acids, pseudo-peptide bonds, and cyclization. Curr. Pharm. Des. 2010, 16 (28), 3185-203.

93. Thapa, P., et al., The Emergence of Cyclic Peptides: The Potential of Bioengineered Peptide Drugs. Int. J. Pept. Res. Ther. 2014, 20 (4), 545-551. 
94. Driggers, E. M., et al., The exploration of macrocycles for drug discovery--an underexploited structural class. Nat. Rev. Drug Discov. 2008, 7 (7), 608-24.

95. Shaabani, S., et al., A patent review on PD-1/PD-L1 antagonists: small molecules, peptides, and macrocycles (2015-2018). Expert Opin Ther Pat 2018, 28 (9), 665-678.

96. Kundu, R., et al., Hybrid Organic-Inorganic Inhibitors of a PDZ Interaction that Regulates the Endocytic Fate of CFTR. Angew. Chem. Int. Ed. Engl. 2012, 51 (29), 7217-7220.

97. Richelle, G. J. J., et al., General and Facile Route to Isomerically Pure Tricyclic Peptides Based on Templated Tandem CLIPS/CuAAC Cyclizations. Angew. Chem. Int. Ed. Engl. 2017, 57 (2), 501-505.

98. van Maarseveen, J. H.; Timmerman, P., Editorial for the special issue on "constrained peptides". Drug Discov. Today Technol. 2017, 26, 1-2.

99. Bernal, F., et al., Reactivation of the p53 tumor suppressor pathway by a stapled p53 peptide. J. Am. Chem. Soc. 2007, 129 (9), 2456-7.

100. Chorev, M.; Goodman, M., A Dozen Years of Retro-Inverso Peptidomimetics. Acc. Chem. Res. 1993,26 (5), 266-273.

101. Zuckermann, R. N., Peptoid origins. Biopolymers 2011, 96 (5), 545-55.

102. Kessler, H., Peptoids - a New Approach to the Development of Pharmaceuticals. Angew. Chem. Int. Ed. Engl. 1993, 32 (4), 543-544.

103. Bohacek, R. S.; Mcmartin, C., Multiple Highly Diverse Structures Complementary to Enzyme Binding-Sites - Results of Extensive Application of a De-Novo Design Method Incorporating Combinatorial Growth. J. Am. Chem. Soc. 1994, 116 (13), 5560-5571.

104. Floris, M.; Moro, S., Mimicking Peptides... In Silico. Mol. Inform. 2012, 31 (1), 12-20.

105. Floris, M., et al., Swimming into peptidomimetic chemical space using pepMMsMIMIC. Nucleic Acids Res. 2011, 39, W261-W269.

106. Bond, G. L., et al., MDM2 is a central node in the p53 pathway: 12 years and counting. Curr. Cancer Drug Targets 2005, 5 (1), 3-8.

107. Kussie, P. H., et al., Structure of the MDM2 oncoprotein bound to the p53 tumor suppressor transactivation domain. Science 1996, 274 (5289), 948-53.

108. Nayak, S. K., et al., p53-Mdm2 Interaction Inhibitors as Novel Nongenotoxic Anticancer Agents. Curr Cancer Drug Targets 2018, 18 (8), 749-772.

109. Ding, K., et al., Structure-based design of potent non-peptide MDM2 inhibitors. J. Am. Chem. Soc. 2005, 127 (29), 10130-1.

110. Nguyen, M., et al., Small molecule obatoclax (GX15-070) antagonizes MCL-1 and overcomes MCL-1mediated resistance to apoptosis. Proc Natl Acad Sci U S A 2007, 104 (49), 19512-7.

111. Oltersdorf, T., et al., An inhibitor of Bcl-2 family proteins induces regression of solid tumours. Nature 2005, 435 (7042), 677-81.

112. Tse, C., et al., ABT-263: a potent and orally bioavailable Bcl-2 family inhibitor. Cancer Res 2008, 68 (9), 3421-8.

113. Souers, A. J., et al., ABT-199, a potent and selective BCL-2 inhibitor, achieves antitumor activity while sparing platelets. Nat Med 2013, 19 (2), 202-8.

114. Cohen, N. A., et al., A competitive stapled peptide screen identifies a selective small molecule that overcomes MCL-1-dependent leukemia cell survival. Chem Biol 2012, 19 (9), 1175-86.

115. Gavathiotis, E., et al., Direct and selective small-molecule activation of proapoptotic BAX. Nat Chem Biol 2012, 8 (7), 639-45.

116. Tuszynski, G. P.; Cossu, G., Differential cytotoxic effect of gossypol on human melanoma, colon carcinoma, and other tissue culture cell lines. Cancer Res 1984, 44 (2), 768-71.

117. Wei, J., et al., Apogossypol derivatives as antagonists of antiapoptotic Bcl-2 family proteins. Mol Cancer Ther 2009, 8 (4), 904-13.

118. Vaux, D. L., et al., Bcl-2 gene promotes haemopoietic cell survival and cooperates with c-myc to immortalize pre-B cells. Nature 1988, 335 (6189), 440-2.

119. Sattler, M., et al., Structure of Bcl-xL-Bak peptide complex: recognition between regulators of apoptosis. Science 1997, 275 (5302), 983-6.

120. Lomonosova, E.; Chinnadurai, G., BH3-only proteins in apoptosis and beyond: an overview. Oncogene 2008, 27 Suppl 1, S2-19.

121. Berman, H. M., et al., The Protein Data Bank. Nucleic Acids Res. 2000, 28 (1), 235-42.

122. Liou, J. W., et al., In silico analysis reveals sequential interactions and protein conformational changes during the binding of chemokine CXCL-8 to its receptor CXCR1. PLoS One 2014, 9 (4), e94178.

123. Koenen, R. R., et al., Disrupting functional interactions between platelet chemokines inhibits atherosclerosis in hyperlipidemic mice. Nat. Med. 2009, 15 (1), 97-103.

124. Hermeking, H.; Benzinger, A., 14-3-3 proteins in cell cycle regulation. Semin. Cancer Biol. 2006, 16 (3), 183-92.

125. Ballone, A., et al., 14-3-3: A Case Study in PPI Modulation. Molecules 2018, 23 (6). 
126. Andrei, S. A., et al., Inhibition of 14-3-3/Tau by Hybrid Small-Molecule Peptides Operating via Two Different Binding Modes. ACS Chem. Neurosci. 2018.

127. Andrei, S. A., et al., Rationally Designed Semisynthetic Natural Product Analogues for Stabilization of 143-3 Protein-Protein Interactions. Angew Chem Int Ed Engl 2018, 57 (41), 13470-13474.

128. Chou, C. C., et al., KCa3.1: target and marker for cancer, autoimmune disorder and vascular inflammation? Expert Rev. Mol. Diagn. 2008, 8 (2), 179-87.

129. Zhang, M., et al., Identification of the functional binding pocket for compounds targeting small-conductance $\mathrm{Ca}(2)(+)$-activated potassium channels. Nat. Commun. 2012, 3, 1021.

130. Chen, J.; Kriwacki, R. W., Intrinsically Disordered Proteins: Structure, Function and Therapeutics. J. Mol. Biol. 2018, 430 (16), 2275-2277.

131. Collepardo-Guevara, R., et al., Chromatin Unfolding by Epigenetic Modifications Explained by Dramatic Impairment of Internucleosome Interactions: A Multiscale Computational Study. J. Am. Chem. Soc. 2015, 137 (32), 10205-15.

132. Chong, S. H., et al., Computer Simulations of Intrinsically Disordered Proteins. Annu. Rev. Phys. Chem. 2017, 68, 117-134.

133. Huang, J.; MacKerell, A. D., Jr., Force field development and simulations of intrinsically disordered proteins. Curr. Opin. Struct. Biol. 2018, 48, 40-48.

134. Kmiecik, S., et al., Coarse-Grained Protein Models and Their Applications. Chem. Rev. 2016, 116 (14), 7898-936.

135. Baaden, M.; Marrink, S. J., Coarse-grain modelling of protein-protein interactions. Curr. Opin. Struct. Biol. 2013, 23 (6), 878-86.

136. Zinzalla, G., Targeting MYC: is it getting any easier? Future Med. Chem. 2016, 8 (16), 1899-1902.

137. Soucek, L., et al., Modelling Myc inhibition as a cancer therapy. Nature 2008, 455 (7213), 679-683.

138. Harvey, S. R., et al., Small-Molecule Inhibition of c-MYC:MAX Leucine Zipper Formation Is Revealed by Ion Mobility Mass Spectrometry. J. Am. Chem. Soc. 2012, 134 (47), 19384-19392.

139. Yin, X. Y., et al., Low molecular weight inhibitors of Myc-Max interaction and function. Oncogene 2003, 22 (40), 6151-6159.

140. Sammak, S.; Zinzalla, G., Targeting protein-protein interactions (PPIs) of transcription factors: Challenges of intrinsically disordered proteins (IDPs) and regions (IDRs). Prog. Biophys Mol. Biol. 2015, 119 (1), 41 6.

141. Michel, J.; Cuchillo, R., The impact of small molecule binding on the energy landscape of the intrinsically disordered protein C-myc. PLoS One 2012, 7 (7), e41070.

142. Savino, M., et al., The action mechanism of the Myc inhibitor termed Omomyc may give clues on how to target Myc for cancer therapy. PLoS One 2011, 6 (7), e22284.

143. Soucek, L., et al., Omomyc, a potential Myc dominant negative, enhances Myc-induced apoptosis. Cancer Res. 2002, 62 (12), 3507-10.

144. Kumar, D., et al., Therapeutic Interventions of Cancers Using Intrinsically Disordered Proteins as Drug Targets: c-Myc as Model System. Cancer Informatics 2017, 16.

145. Metallo, S. J., Intrinsically disordered proteins are potential drug targets. Curr. Opin. Chem. Biol. 2010, 14 (4), 481-8.

146. Tsafou, K., et al., Targeting Intrinsically Disordered Transcription Factors: Changing the Paradigm. J. Mol. Biol. 2018, 430 (16), 2321-2341.

147. Chen, C. Y.; Tou, W. I., How to design a drug for the disordered proteins? Drug Discov. Today 2013, 18 (19-20), 910-5.

148. Salomon-Ferrer, R., et al., Routine Microsecond Molecular Dynamics Simulations with AMBER on GPUs. 2. Explicit Solvent Particle Mesh Ewald. J. Chem. Theory Comput. 2013, 9 (9), 3878-88.

149. Gotz, A. W., et al., Routine Microsecond Molecular Dynamics Simulations with AMBER on GPUs. 1. Generalized Born. J. Chem. Theory Comput. 2012, 8 (5), 1542-1555. 



\section{Chapter}

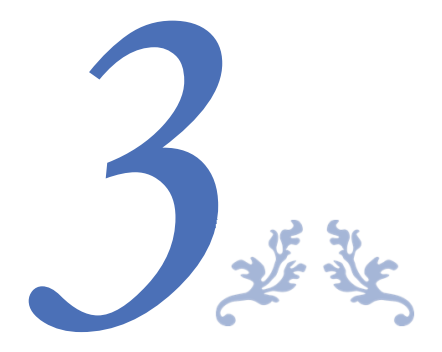

\section{PAD4 takes charge during neutrophil activation: impact of PAD4 mediated NET formation on immune-mediated disease}

Xiaosong Liu1, ${ }^{1,}$, Tom Arfman ${ }^{2,}$, Kanin Wichapong1 ${ }^{1}$, Chris P. M. Reutelingsperger ${ }^{1}$, Jan Voorberg ${ }^{2}$, Gerry A. F. Nicolaes ${ }^{1}$

1Department of Biochemistry, Cardiovascular Research Institute Maastricht (CARIM), Maastricht University, Maastricht, The Netherlands

2Department of Molecular and Cellular Haemostasis, Sanquin Research and Landsteiner Laboratory, Amsterdam, The Netherlands

${ }^{*}$ These authors contributed equally 


\begin{abstract}
Peptidyl arginine deiminase 4 (PAD4) is an enzyme expressed in neutrophils that, when activated, can drive the formation of neutrophil extracellular traps (NETs). Uncontrolled activation of PAD4 and subsequent citrullination of proteins is increasingly recognized as a driver of (auto) immune diseases. Currently, our understanding of PAD4 structure-function relationships and activity control in vivo is incomplete. In this review, employed molecular modelling to generate a three-dimensional structure of the complete PAD4 molecule. Using our model we discuss the catalytic conversion of the arginine substrate to citrulline. Besides mechanistic insight into PAD4 function, we give an overview of biological functions of PAD4 and mechanisms that influence its activation. In addition, we discuss the crucial role of PAD4 mediated citrullination of histones in the formation of neutrophil extracellular traps (NET). Subsequently we focus on the role of PAD4 mediated NET formation and its role in pathogenesis of rheumatoid arthritis, sepsis and (immune-)thrombosis. Finally, we summarize current efforts to design different classes of PAD4 inhibitors that are being developed for improved treatment of autoimmune disorders as well as thrombo-inflammatory disease.
\end{abstract}

Keywords: Protein Arginine Deiminase, PAD4, Citrullination, PAD4 inhibitors, NETosis, Neutrophil extracellular traps (NETs), Rheumatoid Arthritis (RA), Immune disease, Thrombosis, Sepsis, Cancer 


\subsection{Introduction}

Post translational modification (PTM) of amino acids through the addition or removal of chemical groups greatly increases the level of regulation and diversity of protein function ${ }^{1}$. Over the last two decades citrullination, or deimination, has been increasingly recognized as a modifier of immune-mediated disorders. Citrullination is the irreversible conversion of an arginine to citrulline, which is catalyzed by a group of enzymes that belong to the peptidyl arginine deiminase (PAD) family. The proposed chemical modification of arginine into a citrulline by PAD42 is described in Figure 1.
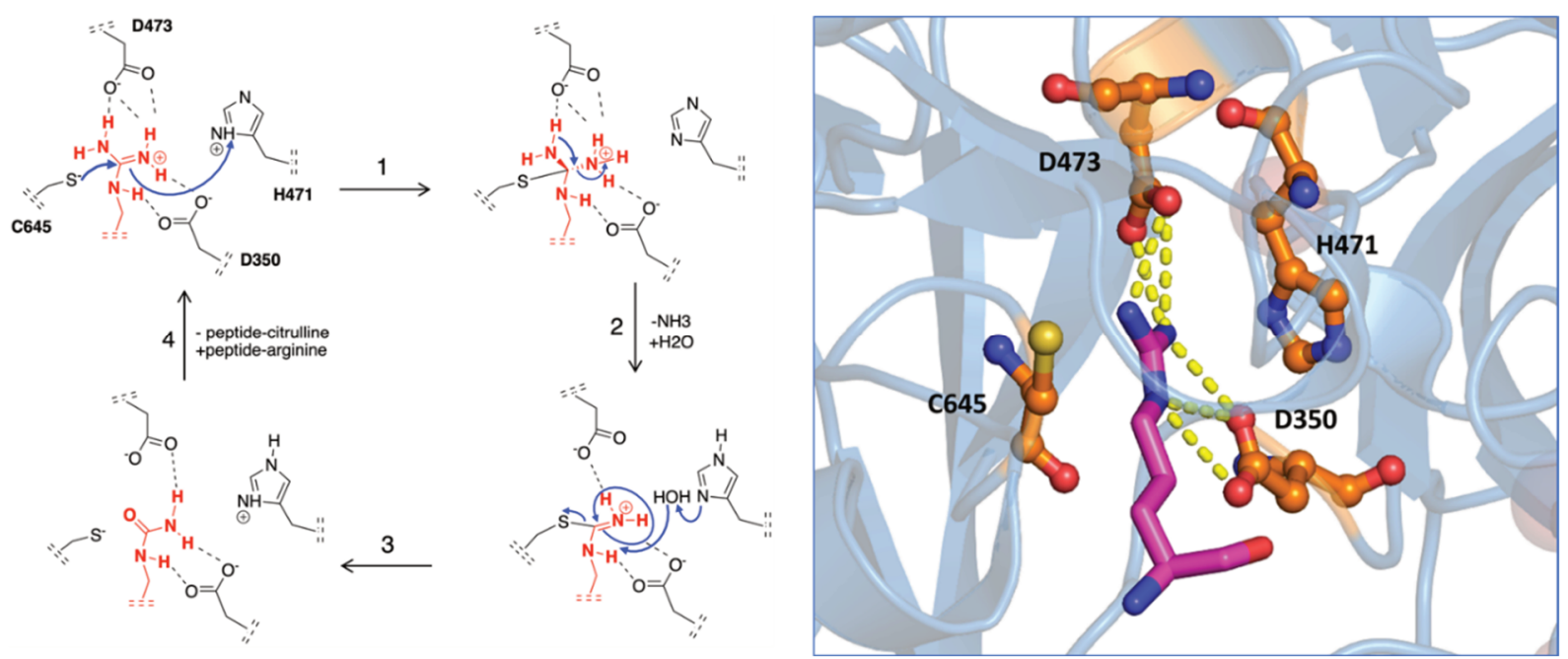

Figure 1. Chemical modification of arginine to citrulline by PAD4. The mechanism of citrullination by PAD4, as adapted from Knuckley et al ${ }^{2}$. The PAD4 residues D350 and D473 function as "anchor points" and sit at the bottom of the tunnel, forming strong interactions via two salt bridges with the guanidinium group of the arginine substrate. The actual catalytic process of deimination is initiated by nucleophilic attack of thiolate-form C645 on the guanidinium carbon of arginine, followed by stabilization of the tetrahedral intermediate through protonation by H471(1). Subsequent collapse of the intermediate (2) yields free ammonia and an S-alkylthiouronium intermediate. This intermediate is then hydrolyzed by a water molecule yielding the original C645 thiolate and citrulline (3). Release of the peptide-citrulline product finishes the catalytic cycle (4). On the right, the crystal structure of PAD4 together with the substrate arginine is shown. The PAD4 active site resides are shown in orange, arginine is shown in magenta. Yellow dotted lines represent hydrogen bonds between arginine and aspartic acids D350 and D473.

Citrullination can greatly affect proteins, mainly due to the fact that the positive charge of arginine is lost when it is converted to a citrulline ${ }^{3}$. This way, citrullination can introduce novel protein structures, functions and interactions as summarized in Figure 2. When citrullination alters the surface electrostatic potential of a protein, its inter/intra-protein interactions can be affected ${ }^{4}$. Likewise, protein dysfunction or unfolding may occur ${ }^{5}$. Importantly, citrullination of proteins has been linked to the formation of autoimmune antibodies in several diseases, including rheumatoid arthritis (RA) ${ }^{6}$.

In humans, five PAD isotypes are expressed: PAD1-4 and PAD6. PADs are highly conserved among different isotypes as well as among different species ${ }^{7}$. For example, PADs share approximately 50-55\% sequence identity within a single species, and each PAD isotype exhibits $70-95 \%$ identity among different species. The high percentage of sequence similarity and identity of human PADs isoforms suggests that all human PAD isotypes have a similar tertiary structure. Moreover, the high conservation 
of amino acids at the active site indicates that these PADs catalyze the conversion of arginine to citrulline via the same mechanism.

The focus of this article, human PAD4, was first described in HL-60 cells and was found to participate in differentiation of HL-60 cells into granulocytes and monocytes/macrophages ${ }^{8}$. PAD4 is the only PAD isotype that carries a nuclear localization signal ${ }^{9}$ and is highly expressed in immune cells including granulocytes, monocytes, macrophages ${ }^{8}, \mathrm{CD}_{34}(+)$ bone marrow cells and multi-potent progenitor cells ${ }^{10}$. PAD4 mainly targets histones in the nucleus ${ }^{11}$, but other proteins, such as glycogen

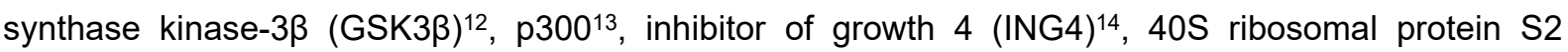
$(\mathrm{RPS} 2)^{15}$, and a disintegrin and metalloproteinase with a thrombospondin type 1 motif, member 13 (ADAMTS13) ${ }^{16}$ have been reported to be citrullinated by PAD4. Moreover, more than 100 different PAD4 substrates were identified in HEK293T cells via mass spectrometric analysis, revealing a rather promiscuous substrate specificity for PAD4 with a modest preference for an RGG motif ${ }^{17}$. Generally, for PAD4 the citrullination turnover rate is inversely correlated with the structural order of the substrate ${ }^{18}$. The only substrate-specific limitation for citrullination seems to be the requirement for a small side chain at the R-2 position, to prevent steric inhibition ${ }^{19}$.

\section{Potential results of protein citrullination}

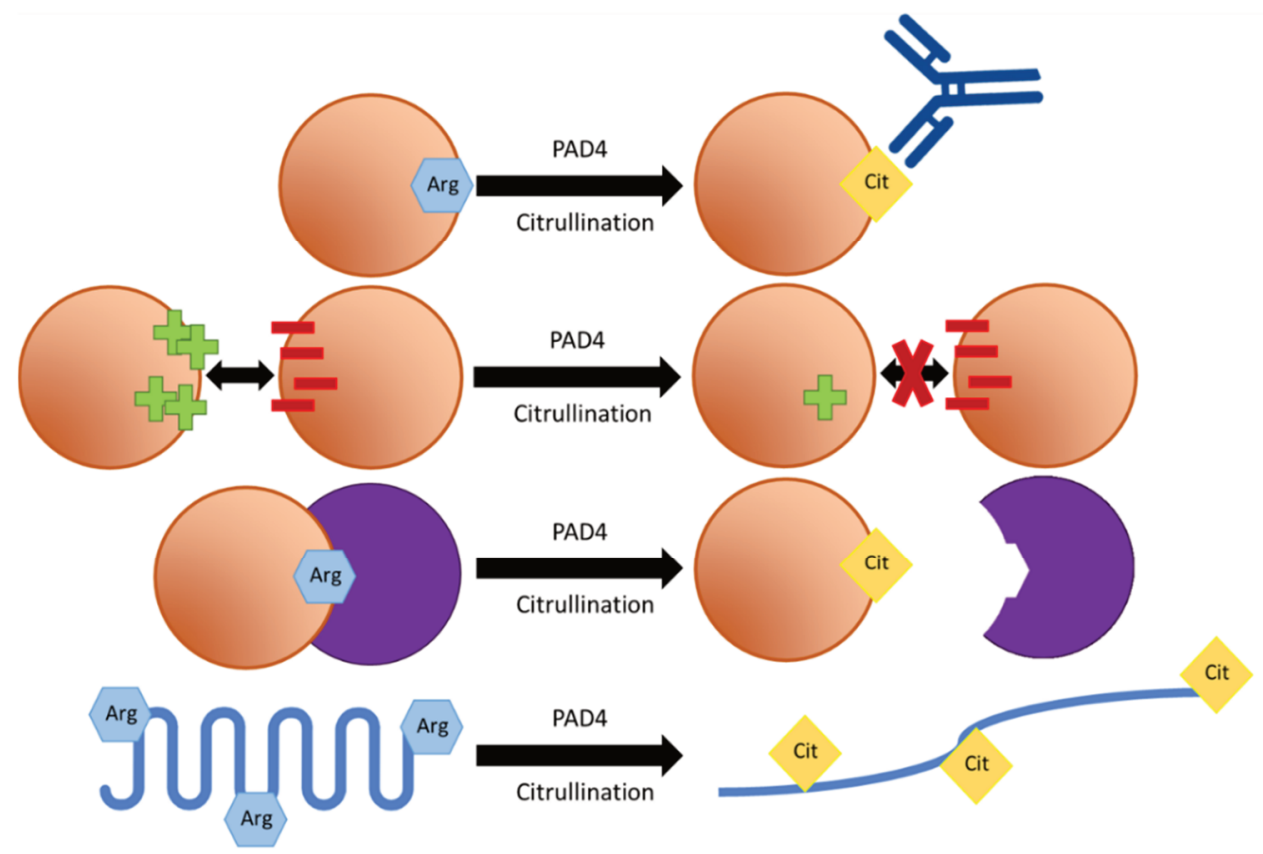

Example

Citrullinated autoantigens in Rheumatoid Arthritis[71]

Histones and DNA in NET formation [55]

Antithrombin[4]

Filaggrin unfolding[5]

Figure 2. Consequences of citrullination. Overview of the possible consequences of citrullination. An orange circle represents a protein, a purple circle represents a protein interactor. For each mechanism, an example is given on the right. Arginine residues are shown as blue hexagons. Citrulline residues are shown as yellow diamonds.

The aforementioned citrullination of nuclear histones by PAD4 is recognized as a trigger in the formation of neutrophil extracellular traps (NETs). NETs have recently been discovered as an integral part of the innate immune system ${ }^{20}$. NETs have the capacity to enhance immune responses by trapping and killing bacteria. However, at the same time, NETs are indiscriminate with respect to their cytotoxicity, and 
uncontrolled NET formation contributes to damage of healthy tissue, which is why PAD4 has been (referred to) described as a 'double-edged sword'21, 22. In neutrophils, different stimuli can result in the formation of NETs, which are produced in distinct cellular pathways ${ }^{23}, 24$. Unlike the classic NETosis pathway, PAD4 activation by calcium results in ROS-independent NET formation, in a process that has been named Leukotoxic Hypercitrullination $(\mathrm{LTH})^{23}$. More and more studies have connected PAD4 or LTH to human diseases, including RA, sepsis, thrombosis and cancer ${ }^{25-29}$.

While the role of PAD4 in the development of disease is being increasingly uncovered, a complete mechanistic insight into factors that are required for the expression and regulation of PAD4 activity is lacking. In this review, we summarize the available PAD4 structure-function data, discuss physiologic functions and provide an insight into mechanisms of PAD4 activation and regulation. Moreover, we provide an overview of the role of PAD4 in the pathogenesis of thrombo-inflammatory and autoimmune disorders. We provide a complete structural overview of PAD4 as a scaffold which may be useful for the development of novel classes of inhibitors to more efficiently combat PAD4-mediated diseases.

\subsection{The structure of PAD4}

The human PADI4 gene of 55,806 bases encodes for a 663 amino acid PAD4 monomer of $74 \mathrm{kDa}$. A PAD4 monomer consists of two domains: a N-terminal domain (residues Met1-Pro300) and a C-terminal catalytic domain (residues Asn301-Pro663). The $\mathrm{N}$-terminal domain can be divided into two immunoglobulin-like subdomains (subdomain I: residues Met1-Cys118, and subdomain II: residues Ala119-Pro300) $)^{30}$ (Figure 3A). The immunoglobulin-like domains are involved in different functions including cell-cell recognition, cell-surface receptors, and have been reported to serve functions in the immune system ${ }^{31}$. The catalytic domain contains the active site and forms an $\alpha / \beta$ propeller structure ${ }^{30}$. Human PAD4 contains five $\mathrm{Ca}^{2+}$ binding sites. $\mathrm{Ca} 1$ and $\mathrm{Ca} 2$ are located in the $\mathrm{C}$-terminal domain and are structurally involved in the calcium-induced conformational changes that occur when the functional active site cleft is generated ${ }^{30}$. Ca3, $\mathrm{Ca} 4$ and $\mathrm{Ca} 5$ are non-catalytic calcium ions situated in the Nterminal domain and are thought to be required for stabilization of the active conformation of PAD4 ${ }^{32}$. At the $\mathrm{N}$-terminus, there is a single nuclear localization signal (NLS) (Figure 3A), which is a (56PPAKKKST-63) motif where three continuous lysine residues 59-61 follow two proline residues ${ }^{9}$. We have employed the Homology Model suite implemented in YASARA program ${ }^{33}$ to build the regions that are missing from the reported incomplete X-ray structures of PAD4 in order to obtain a complete 3D structure of human PAD4. From our homology model it shows that the NLS adopts a loop conformation (Figure $3 \mathrm{~A}$ ) which is responsible for interaction with nuclear transport receptors to guide the passage of PAD4 across the nuclear membrane ${ }^{9}$. Under physiological conditions, PAD4 has a head to tail homodimeric conformation, and it was demonstrated that dimeric PAD4 expresses full catalytic activity and calcium binding ${ }^{30}$. The dimerization of PAD4 is mediated by several electrostatic interactions of which those between R8 from one subunit and D547 from another subunit is most important, as was concluded from mutagenesis studies ${ }^{34}$. Dissociation of dimer formation could reduce PAD4 enzymatic activity and calcium-binding cooperativity. Monomeric PAD4 has a residual $25-50 \%$ enzyme activity, as compared to the dimeric enzyme ${ }^{34}$. 
The PAD4 active site contains a negatively charged U-shaped tunnel which allows the argininecontaining substrates to bind through a so-called "front door" (Figure 3B) ${ }^{35}$. After catalysis, reaction products are released from the active site and leave the active site region through a so-called "back door" (Figure 3B) $)^{35}$. Enzymes with substrate tunnels generally have high substrate selectivity, influenced by the depth and shape of the tunnel, with which they may select particular substrates of an appropriate chain length ${ }^{36}$. The catalytic site is mainly formed by four active site residues (D350, H471, D473, C645) that are deeply buried in the tunnel (Figure 3B) ${ }^{30}$. The conformation of arginine in the active site, flanked by $\mathrm{C} 645$ and $\mathrm{H} 471$ is displayed in Figure 3B.

A
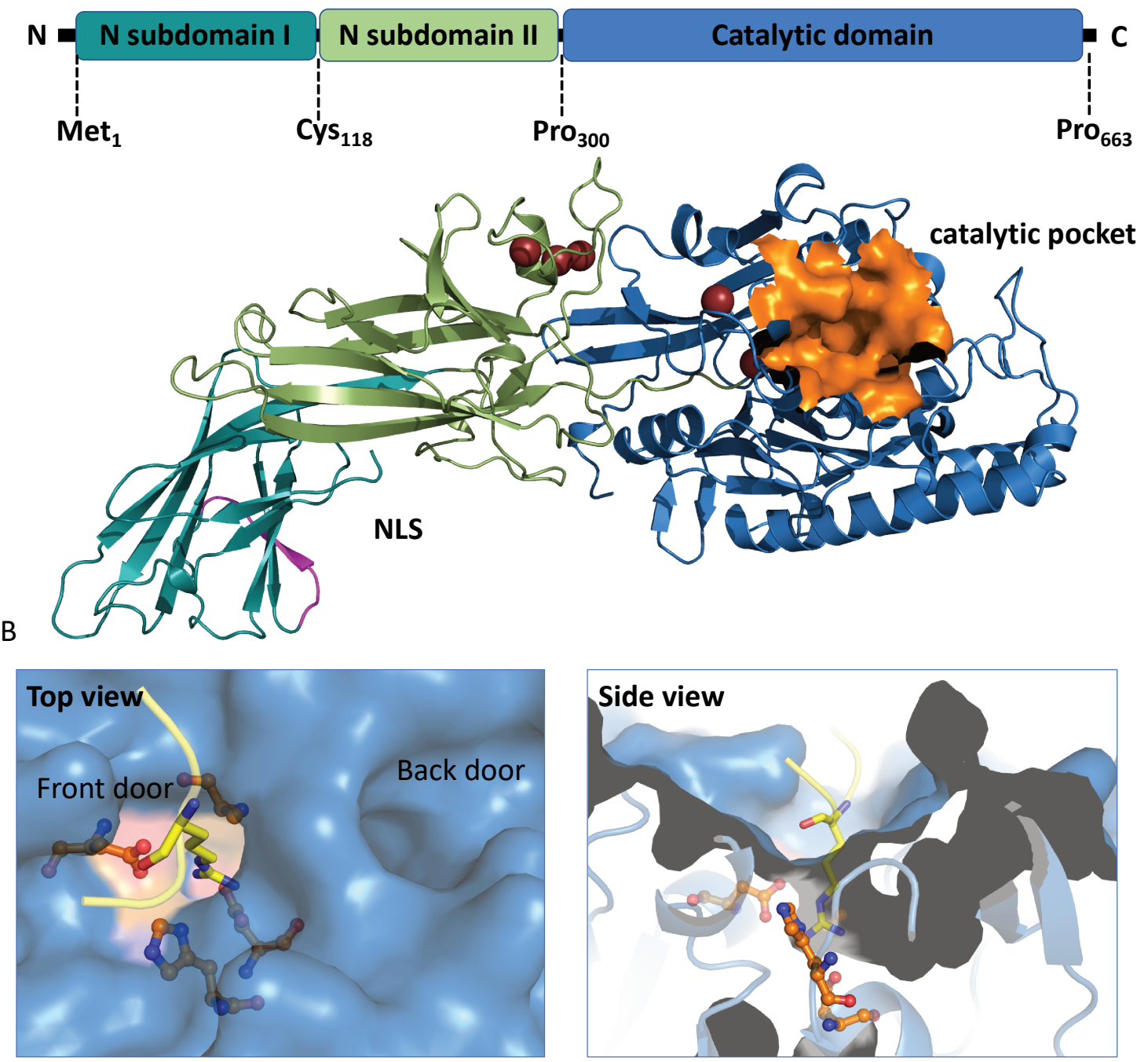

Figure 3. The 3D structure of PAD4. A, full length PAD4 monomer. The homology model of PAD4 is made using YASARA software based on the 3B1U PDB template. The NLS is shown in magenta, and the catalytic pocket is shown in orange. $B$, the catalytic site and substrates binding pocket of PAD4. The arginine substrate containing histone tail (TARKS) is shown in yellow, active site residues are orange. The left pane shows the top view, where the TAKS residues of the histone tails are not shown. The front door and back door of the active site are indicated in the image. The right pane contains the side view of the binding pocket, where it is clear how only the substrate arginine protrudes into the active site. 


\subsection{Activation and regulation of PAD4 activity}

Our understanding of the mechanisms that govern PAD4 activation and regulation is incomplete. Here we provide an overview of factors or conditions that are known to contribute to the up or down-regulation of PAD4 enzyme activity which have been graphically summarized in Figure 4.

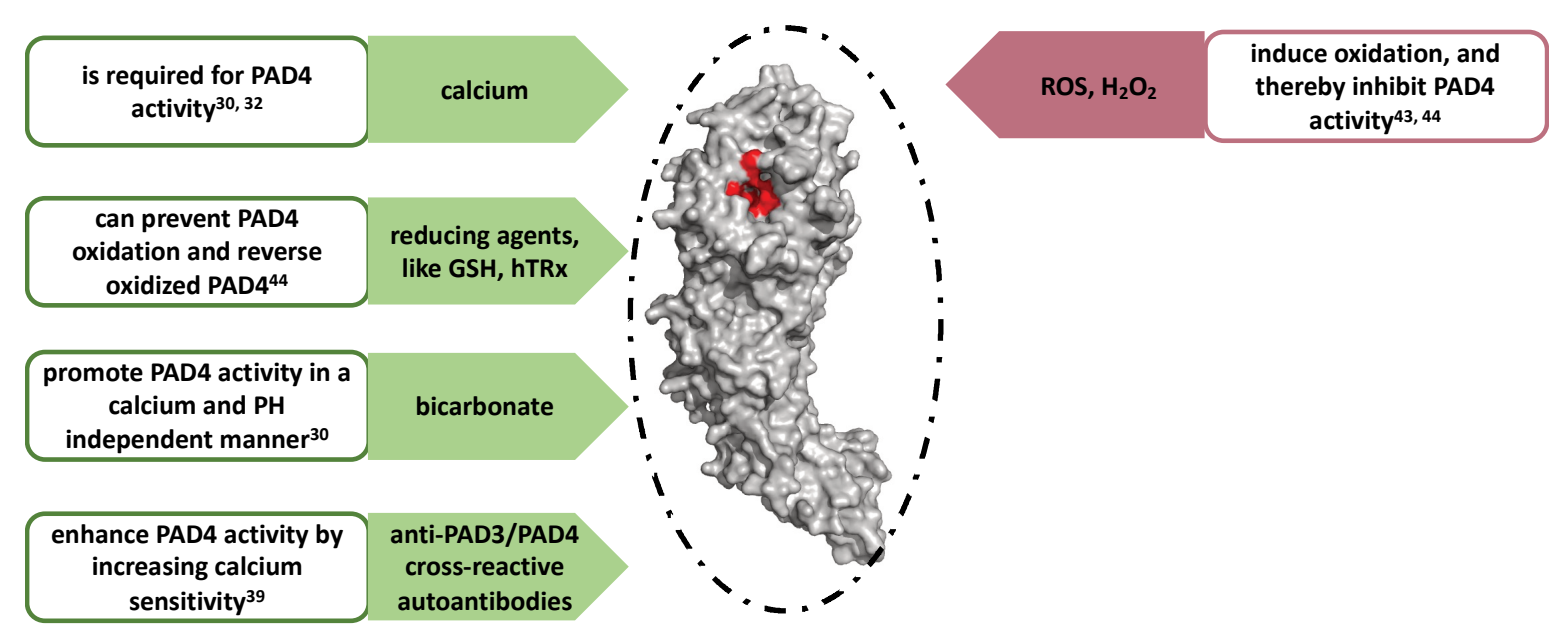

Figure 4. Activation and regulation of PAD4. In the center, the PAD4 crystal structure is given in grey. The active site residues have been given a red color. PAD4 activating mechanisms are given in green arrows. PAD4 inhibitory processes are given in the red arrow.

\subsubsection{Calcium}

As previously introduced, PAD4 is activated intracellularly following a $\mathrm{Ca}^{2+}$ wave. Calcium ionophores, such as lonomycin and A23187 are therefore frequently used to activate PAD4 in vitro ${ }^{37}$. Calcium is known as a key regulator of PAD4 catalytic activity by its capacity to induce structural changes in PAD4. These conformational changes accompany the transition of an inactive to an active PAD4 conformation ${ }^{30,32}$. To achieve maximal activation in vitro, PAD4 requires typically 5-10 mM calcium to occupy its five calcium sites which can be recognized from the PAD4 structure as described above $\mathrm{e}^{30,32}$. However, intracellular calcium concentration range from $10 \mathrm{nM}-100 \mu \mathrm{M}$ and the calcium concentration in blood ranges from 2.2-2.6 mM $\mathrm{m}^{38}$. This suggests that in vivo, other factors than calcium play a role in PAD4 activation.

\subsubsection{Anti-PAD3/PAD4 cross-reactive antibodies}

It has been suggested that cofactors may lower the requirement of PAD4 for calcium and assist in activation of PAD4, although this is, as of yet, a poorly understood mechanism. One example where the threshold for PAD4 activation by calcium is lowered is present in some RA patients who produce antibodies that react with both PAD3 and PAD439. Compared with patients who only have anti-PAD4 antibodies, patients with combined anti-PAD3/PAD4 cross-reactive antibodies have the most erosive joint disease. Darrah E et al. showed that the anti-PAD3/PAD4 cross-reactive antibodies can activate and increase the catalytic efficiency of PAD4 at low calcium concentrations $(0.2 \mathrm{mM})$. It was hypothesized that stabilization of the active conformation of PAD4 was achieved by binding of cross- 
reactive antibodies in a calcium-independent fashion ${ }^{39}$. However, conclusive data supporting this mechanism is currently lacking.

\subsubsection{Bicarbonate}

Furthermore, independent of calcium concentration, it was shown that bicarbonate increases PAD4 activity, without altering the affinity of PAD4 for calcium ${ }^{40}$. In the presence of bicarbonate, an increase of PAD4 catalyzed citrullination of histone $\mathrm{H} 3$ and fibrinogen was described. However, the in vitro determined optimal bicarbonate concentration for the modulation of PAD4 activity was around 22 mM. This concentration favors the activity of PAD4 in the extracellular environment, given that the bicarbonate concentration in human serum is $17-29 \mathrm{mM}$ and inside cells it is $<10 \mathrm{mM}^{41}$. How exactly bicarbonate can modulate PAD4 catalytic activity is still unknown, and further experimental studies are needed to understand its mode of action.

\subsubsection{Redox regulation}

As early as in 1991 it was shown that three mouse PADs isoforms (PAD1-3) require a reducing environment for their enzymatic activity42. PADs can be rapidly oxidized and become inactive after release into the extracellular space, and both $\mathrm{H}_{2} \mathrm{O}_{2}$ and Reactive Oxygen Species (ROS) formation can inhibit PAD activity in a dose dependent manner ${ }^{43}, 44$. Most likely, this requirement for a reducing environment for PAD4 activity is due to the C645 residue requiring reduction to the thiolate form to allow nucleophilic attack of the guanidinium carbon of arginine (Figure 1). Reducing agents, such as DTT (dithiothreitol), TCEP (Tris(2-carboxyethyl) phosphine) or beta-mercaptoethanol, are used in vitro to preserve PAD4 activity. The intracellular environment is also regarded as a reducing environment. Damgaard et al. showed that lower millimolar range GSH as present in the cytoplasm was capable of activating PAD4 in an in vitro assay ${ }^{44}$. However, the extracellular level of $\mathrm{GSH}(<1 \mathrm{mM})$ is too low to prevent the oxidation of PAD4. Additionally, elevation of the oxidoreductase enzyme thioredoxin was observed in RA synovial fluid and plasma, and Nagar et al. proved the PAD4 activating capacity of thioredoxin at physiological concentration ${ }^{45}$. Taken together, the redox state of the PAD4 environment may contribute to the regulation of PAD4 activity under physiological conditions.

\subsubsection{PAD4 auto-citrullination}

A further candidate for control of PAD4 activation is the process of auto-citrullination. PAD4 has been shown to be capable of citrullinating itself on several positions, which was shown to affect PAD4 proteinprotein interactions ${ }^{46}$. Regarding the effect of auto-citrullination on PAD4 activity, we showed in a recent publication that PAD4 autocitrullination has no effect on its activity ${ }^{47}$.

\subsection{PAD4 in NET formation}

Neutrophils function as a first line of defense of the human innate immune system ${ }^{48}$. They migrate to sites of infection from the blood to engulf and kill invasive pathogens like bacteria, fungi, and viruses ${ }^{49}$. In response to an inflammatory environment neutrophil can respond in several ways. More established are the NETs which neutrophils form as part of the innate immune response. NETs were first characterized in 2004 as a new mechanism of neutrophil activation in response to pathogens ${ }^{50}$. 
Decondensed chromatin is regarded as the scaffold of NETs, which is decorated with granular and cytoplasmic proteins, including neutrophil elastase (NE), myeloperoxidase (MPO), cathepsin G, as well as histones ${ }^{20}$. Recently, Thiama et al. used high-resolution microscopy to unravel the sequence of events during neutrophil extracellular trap formation (NETosis). They report that following stimulation, NET forming neutrophils quickly disassemble their actin cytoskeleton and start shedding plasma membrane microvesicles. Subsequent remodeling of the vimentin and microtubule cytoskeletons precedes ER vesiculation. This is followed by chromatin decondensation and loss of nuclear lobularity, after which permeabilization of the nuclear envelope and plasma membrane occurs. Lastly, chromatin exits through the ruptured nuclear envelope into the cytoplasm, and enters the extracellular matrix after rupture of the plasma membrane ${ }^{51}$. Interestingly, NETs can be formed as a result of several pathways. Initially, NETosis was reported as a mechanism that depends on reactive oxygen species (ROS) generated by neutrophil NADPH oxidase ${ }^{52}$. In this mechanism, ROS production leads to destabilization of granule membranes and the nuclear envelope, which allows mixing of cytoplasmic and nuclear components. Cytoplastic NE can subsequently cleave the nuclear histones, which results in a decondensation of chromatin. However, it was discovered that inhibition of NADPH oxidase cannot prevent NET formation in response to the bacteria ${ }^{53}$, hinting at an alternative NET formation pathway ${ }^{50,54}$.

Recent studies have revealed that PAD4-catalyzed histone citrullination is a crucial step for NET formation in a ROS independent pathway. During PAD4-mediated NET formation, or leukotoxic hypercitrullination ${ }^{23}$, PAD4 citrullinates histones in neutrophils. Citrullinated histones lose positive surface charges that interact with the negatively charged DNA backbone, which results in decondensation of chromatin ${ }^{55}$. Non-DNA bound histones can subsequently break the nuclear envelope and plasma membranes ${ }^{56}$. The inability for PAD4-deficient mice to form NETs in response to lonomycin shows the critical role PAD4 plays in this process ${ }^{50,57}$. In line with this, inhibition of PAD4 by the known PAD4 inhibitor $\mathrm{Cl}$-amidine significantly reduces histone citrullination and prevents the formation of NETlike structures after stimulation of neutrophils with ionomycin ${ }^{58}$.

As introduced previously, NETs themselves are viewed as a double-edged-sword ${ }^{21,22}$. NETs are known to activate the Complement system through binding of $\mathrm{C} 1 \mathrm{q}$ to extracellular DNA ${ }^{59}$. Additionally, NET's prime $\mathrm{CD} 4^{+} \mathrm{T}$-cells in a process that engages the $\mathrm{T}$-cell receptor, in doing so lowering their activation threshold ${ }^{60}$. Additionally, NETs exacerbate inflammation through danger associated molecular pattern (DAMP) signaling61. Especially extracellular histones resulting from NETs have been shown to damage tissues through activation of Toll-like-receptors 2 and $4^{61}$ and have a pro-thrombotic effect through their capacity to activate platelets ${ }^{62}$. Furthermore, NET's can act as a scaffold for thrombus formation through binding of platelets, red blood cells and procoagulant molecules ${ }^{63}$. In addition to this, extracellular activity of PAD4 has been postulated to be a driver of diseases through uncontrolled citrullination of proteins. As such, NET formation is seen as a link between inflammation and onset of several (autoimmune) diseases $^{64}$, some of which will be discussed in the next paragraph. 


\subsection{Role of PAD4 in pathogenesis of diseases}

Citrullination of proteins by PAD4 is associated with a multitude of disease. Here, we provide a detailed overview of the role of PAD4 in rheumatoid arthritis, sepsis and thrombosis.

\subsubsection{Rheumatoid arthritis}

$\mathrm{RA}$ is one of the most common inflammatory autoimmune diseases which affects around $0.5-1.0 \%$ of the population worldwide ${ }^{65}$. RA patients suffer from systemic inflammation, which leads to damaging of synovial tissues resulting in movement restriction and disability66. In the past decades, a growing number of studies have pointed to dysregulation of PAD4 mediated citrullination of extracellular proteins as a driver of the autoimmune response in $\mathrm{RA}$, as $75 \%$ of patients produce anti-citrullinated protein antibodies (ACPA's). This leads to the working hypothesis that ongoing citrullination in RA synovial fluid provides autoantigens that lead to continuation of the inflammatory response ${ }^{67}$. Associations between genetic variants of PAD4 and increased risk in RA development have been made. Recently, a recent meta-analysis of several studies that focused on the single nucleotide PAD4 polymorphism 104C/T indicated that the polymorphism associates with an increased risk for developing $\mathrm{RA}^{68,69}$. The haplotype of the PADI4 gene that increases RA risk is linked to increased production of citrullinated peptides, further strengthening the connection between citrullination and RA ${ }^{68}$. In 2018, Tivawala et al. published the RA citrullinome, and identified many different citrullinated proteins in synovial fluid of patients with $\mathrm{RA}^{70}$. Citrullinated proteins can be considered autoantigens that recognized as non-self by the immune system which induce autoantibody generation against citrulline-containing proteins ${ }^{71}$. In short, uncontrolled citrullination of proteins can result in the immune system losing tolerance-to-self. This principle was illustrated in a study with rats, which reports that the presence of citrullinated protein and PAD4 levels correlate with the severity of collagen-induced arthritis 6 . PAD4 knockout mice present with a significantly reduced arthritis severity in a glucose-6-phosphate isomerase-induced arthritis model ${ }^{72}$. In a collagen-induced arthritis mouse model it was shown that anti-collagen antibodies and inflammatory cytokine levels were remarkably decreased in PAD4 deficient mice compared to wild type mice ${ }^{27}$. Importantly, ACPA's appear in an early stage of the disease and some are even regarded as a hallmark of RA diagnosis and prediction. As IgG anti-filaggrin autoantibodies can recognize a citrulline epitope of various forms of filaggrin, they are regarded a very specific marker of $\mathrm{RA}^{73}$. Importantly, higher ACPA titers correlate with disease severity ${ }^{74}$. Anti-cyclic citrullinated peptide autoantibodies (anti-CCP) have $\sim 98 \%$ specificity and $68-75 \%$ sensitivity and can be used in early stage RA diagnosis ${ }^{75}$.

\subsubsection{PAD4 in Sepsis}

Sepsis-related deaths account for approximately $20 \%$ of all global deaths, with approximately 50 million reported cases in $2017^{76}$. A growing number of studies have demonstrated that NETs are one of the major contributors to disease severity in sepsis ${ }^{77-79}$. As mentioned above, citrullination of nuclear histones by PAD4 leading to decondensation of chromatin is a key step in the onset of NET formation. NETs are formed within the vasculature during sepsis, in response to circulating pathogens ${ }^{80}$. In the early stages of sepsis, depletion of NETs is not beneficial for the prevention or containment of a systemic infection and it can even exacerbate the pathology, as was observed in a mouse model of polymicrobial 
sepsis $^{81}$. In severe sepsis, NETs and platelets can synergistically participate in pathogen containment. Histones were reported as major mediators of death in sepsis by contributing to endothelial damage and organ failure ${ }^{77}$. Increased histone levels correlated with mortality in sepsis patients and inversely correlated with platelet counts ${ }^{28,82}$. Direct targeting of extracellular histones with histone antibodies, activated protein $\mathrm{C}$ or heparin can significantly reduce mortality in different mouse models of sepsis ${ }^{77}$. Recently it was shown that citrullinated extracellular histone $\mathrm{H} 3$ levels, a significant marker of NET formation ${ }^{83}$, are elevated in wild type mice as compared to PAD4 knockout mice in a pneumonia-derived sepsis model ${ }^{84}$. In a dual insult mouse model of hemorrhagic shock and sepsis, deficiency of PAD4 resulted in decreased organ dysfunction and improved survival as compared with wild-type animals ${ }^{29}$. Therefore, prevention of NET formation by inhibition of PAD4 to block histone release is regarded as an attractive strategy to prevent NET-induced tissue damage in septic patients.

\subsubsection{PAD4 and Thrombosis}

Thromboembolic conditions are currently the leading global cause of mortality ${ }^{85}$. NETs, and by extension PAD4, are known mediators of thrombosis ${ }^{86}$. This was established originally because cleavage of tissue factor pathway inhibitor by NET-bound serine proteases (NE, cathepsin $\mathrm{G}$ ) has a pro-thrombotic effect ${ }^{87}$. Interestingly NETs have been found in both venous and arterial thrombi, where they serve as a scaffold for platelets and red blood cells (RBC), facilitating their adhesion and aggregation ${ }^{88-90}$. Additionally NETs bind plasma proteins including fibronectin and $\mathrm{VWF}^{25}$, further contributing to thrombus formation. Constituents of NETs, primarily extracellular DNA and histones, also profoundly influence coagulation ${ }^{25}$. Levels of these NET biomarkers correlate with disease severity in thrombotic disorders including thrombotic microangiopathies (TMAs) and deep vein thrombosis (DVT). Extracellular DNA leads to thrombin generation in patients with sepsis ${ }^{88}$, and in an experimental DVT model was able to promote thrombosis through induction of platelet aggregation ${ }^{25}$. Extracellular histones released from NETs increase plasma thrombin generation and thereby promote coagulation through TLR2 and TLR4 mediated activation of platelets ${ }^{91}$. Importantly, as NETs are primarily discussed in the context of inflammation, here it has to be mentioned that NETs can be formed in non-inflammatory conditions, such as during exhaustive exercise ${ }^{92}$. This finding, combined with the previously mentioned procoagulant properties of NETs has led researchers to view NET formation as a homeostatic process which must be tightly controlled ${ }^{86}$. Inflammation driven activation of neutrophils, platelets and vascular endothelial cells may promote a state of immune-thrombosis which is most likely driving to disease severity in for example COVID-19 patients ${ }^{93}$.

Since PAD4 is essential for NET formation, an increasing number of studies focus on the involvement of PAD4 in thrombosis. It was found that PAD4 knockout mice generate much less thrombi than wildtype mice after induction of an inferior vena cava stenosis ${ }^{94}$. Depletion of PAD4 significantly reduced neutrophil and platelet accumulation at the areas of vascular injury, thereby preventing venous thrombosis in a murine model of heparin-induced thrombocytopenia ${ }^{26,95}$. PAD4 is a key player in the pathogenesis of thrombosis not only by triggering NET formation but also by citrullinating proteins that are involved in maintaining haemostasis. Evidence has been obtained for PAD4-mediated citrullination of serine protease inhibitors such as antithrombin, as well as fibrinogen and the VWF cleaving protease 
ADAMTS1370. Citrullination of serine protease inhibitors can result in activity loss, which in turn could result in dysregulation of blood coagulation ${ }^{96}$. Citrullination of antithrombin abolished its ability to inhibit thrombin activation, however, increased levels of citrullinated antithrombin found in the plasma of RA and colorectal adenocarcinoma patients did not show association with thrombosis ${ }^{97}$. Additionally, injection of PAD4 in vivo can induce citrullination of plasma proteins like ADAMTS13 (a disintegrin and metalloproteinase with thrombospondin type-1 motif-13) thereby reducing the ability of ADAMTS13 to cleave VWF (von Willebrand factor), resulting in the formation of prothrombotic VWF-platelet strings ${ }^{16}$. Based on these findings we hypothesize that PAD4 dependent citrullination of hemastatic proteins further modulates thrombus formation on the highly prothrombotic three-dimensional scaffold that results from NETosis. Therefore, PAD4 directed inhibitors provide a highly attractive strategy to combat or prevent (immune-)thrombosis.

\subsection{PAD4 inhibition}

The contribution of PAD4 in the pathologies described above is a promising premise for therapeutic use of PAD4 inhibitors. Unfortunately, no physiological PAD4 inhibitors are known that could help guide inhibitor design. Furthermore, design of a PAD4 isotype-specific inhibitor is complicated by the high structural conservation of the PAD active site among all isotypes. Inhibiting several PAD isotypes simultaneously is undesirable, as each isotype is involved in separate biological pathways ${ }^{7}$. An overview of the expression location and targets of each PAD isotype is given in Table 1. Nonetheless, recent years have seen substantial efforts made towards the design of therapeutically usable PAD4 inhibitors, especially by Thompson and colleagues. Both irreversible and reversible PAD4 inhibitors have been explored. Irreversible inhibitors contain a halo (commonly F-, $\mathrm{Cl}$-) acetamidine group which covalently binds to Cys645 in the PAD4 active site (Figure 5B). The first described PAD inhibitor of this class was 2-chloroacetamidine ${ }^{98}$. However, since then multiple generations that improve upon the 2-chloroacetamine characteristics have been developed. Reversible PAD4 inhibitors are chemically more diverse, and rely on different mechanisms of PAD4 inhibition, such as guanidine-containing compounds that interact with PAD4 active site residues (Figure 5C) and compounds that occupy both the "front door" and "back door" of the U-shaped tunnel. This topic is extensively covered in a recent article by Nemmara and Thompson ${ }^{99}$. The different modes of action of irreversible and reversible PAD4 inhibitors are described in Figure 5. In conclusion, it appears very difficult to design PADs inhibitors to have a high selectivity for the PAD4 isotype in combination with achieving high potency. Currently, there is no PAD4 inhibitor with the required characteristics to be used therapeutically. We anticipate that highthroughput screening of small compounds in conjunction with in silico biomolecular modelling will have the potential to design novel classes of high-affinity, highly specific PAD4 inhibitors that can be successfully used for treatment of patients suffering from thrombosis and/or inflammatory disorders. 
Table 1. Expression localization, substrates and related diseases of PAD enzymes

\begin{tabular}{|c|c|c|c|}
\hline PADs & Expression & Substrates & Diseases \\
\hline PAD1 & Epidermis, uterus, keratinocytes, & Keratin, filaggrin & Skin disorder \\
\hline PAD2 & $\begin{array}{c}\text { Muscle, brain, skin, } \\
\text { macrophages, lymphocytes, } \\
\text { rheumatoid arthritis synovium, } \\
\text { salivary glands, epidermis, } \\
\text { astrocytes, spinal cord, spleen, } \\
\text { leukocytes }\end{array}$ & $\begin{array}{c}\text { Myelin basic protein, vimentin, } \\
\beta / \gamma \text {-actin, fibrinogen, histones, } \\
\text { RNAP2-CTD }\end{array}$ & $\begin{array}{l}\text { Inflammation, Rheumatoid } \\
\text { arthritis, multiple sclerosis, } \\
\text { cancer }\end{array}$ \\
\hline PAD3 & Hair follicles, epidermis & $\begin{array}{l}\text { Keratin, Filaggrin, trichohyalin, } \\
\text { apoptosis-inducing factor, } \\
\text { vimentin }\end{array}$ & Skin disorder \\
\hline PAD4 & $\begin{array}{l}\text { Granulocytes, macrophages, } \\
\text { CD34+ cells, multi-potent } \\
\text { progenitor cells, mammary gland } \\
\text { cells, neurons, various tumors, } \\
\text { spleen }\end{array}$ & $\begin{array}{l}\text { Histones, fibrinogen, filaggrin, } \\
\text { NPM1/B23, GSK3 } 3 \text {, RPS2, } \\
\text { myeloperoxidase, ING4, p300, } \\
\text { DNMT3A, NPM1, E2F-1, } \\
\text { antithrombin, ADAMTS13, } \\
\text { Serpins }\end{array}$ & $\begin{array}{l}\text { Inflammation, Rheumatoid } \\
\text { arthritis, multiple sclerosis, } \\
\text { sepsis, deep vein thrombosis, } \\
\text { lupus, cancer, Alzheimer's, } \\
\text { kidney dysfunction }\end{array}$ \\
\hline PAD6 & Eggs, early embryos, ovary & Unknown & Unknown \\
\hline
\end{tabular}

\subsection{Conclusion and Future perspective}

In recent years PAD4, through its crucial role in NET formation, has emerged as a key driver of immune mediated diseases, thrombosis and inflammatory disorders. In this review, we have summarized the state-of-the-art on PAD4 structure, activation, cellular functions and its role in disease. Our knowledge on the structure-function relationship of PAD4 is currently incomplete. A complete experimental structure of PAD4 is not yet available, and we have to partially rely on homology models as presented in this review for the design of novel classes of PAD4 inhibitors.

There are also still many unknowns in our understanding of the mechanisms that control PAD4 activity. Often, non-physiological reducing agents like DTT are used to activate PAD4 in vitro, in combination with $\mathrm{Ca}^{+}{ }^{+}$concentrations that far exceed physiological levels ${ }^{32,45}$. Although hypothesized physiological co-activators of PAD4 are being investigated, there is currently no consensus on how PAD4 reaches full activity within the neutrophil nuclei. Additionally, the observation that PAD4 citrullinates plasma proteins like antithrombin and ADAMTS13 seems to be in direct contradiction to the fact that PAD4 requires a reducing environment to remain active in vitro. Clearly, additional research into the control of PAD4 activity in vivo is crucial to improve our understanding of this enigmatic and promiscuous enzyme.

The double-edged-sword nature of PAD4 facilitated NET formation however is uncontested. The contribution of PAD4 to NET formation currently appears to be the major biological function of PAD4. NETs are capable of neutralizing pathogens, but also exacerbate inflammation and have multiple prothrombotic effects. Taken together, this indicates that NET formation is a balanced process, the full inhibition of which may not be desirable, and a rebalancing of this process could be a better approach to optimally combat disease ${ }^{100}$. Nevertheless, PAD4 qualifies as a target for pharmacological 
intervention. In this review we presented multiple generations of PAD4 inhibitors. However, there currently is no inhibitor that combines PAD4 isotype specificity with high potency. Especially the high conservation in active site structure between the different PAD isoforms makes specificity hard to achieve. Therefore, in order to design novel and specific PAD4 inhibitors, we propose that it is essential to focus on residues at the substrate binding pocket which could form specific interactions with PAD4selective inhibitors. Several residues qualify for such condition, such as R374, which exists in PAD1 and PAD4 but not in PAD2 and PAD3; R639 is only present in PAD4; H640 only exists in PAD4 and PAD3. Disrupting PAD4 dimerization, or blocking of the previously mentioned "back door" rather than "front door" could also be used to derive novel and selective PAD4 inhibitors.

In conclusion, although the structures, functions and inhibitions of PAD4 have been investigated and studied during the last decades, there are still many questions surrounding PAD4 structure and function. Improving our understanding of this remarkable enzyme will pave the way for the development of potent and specific inhibitors, with exciting potential for therapeutic use.
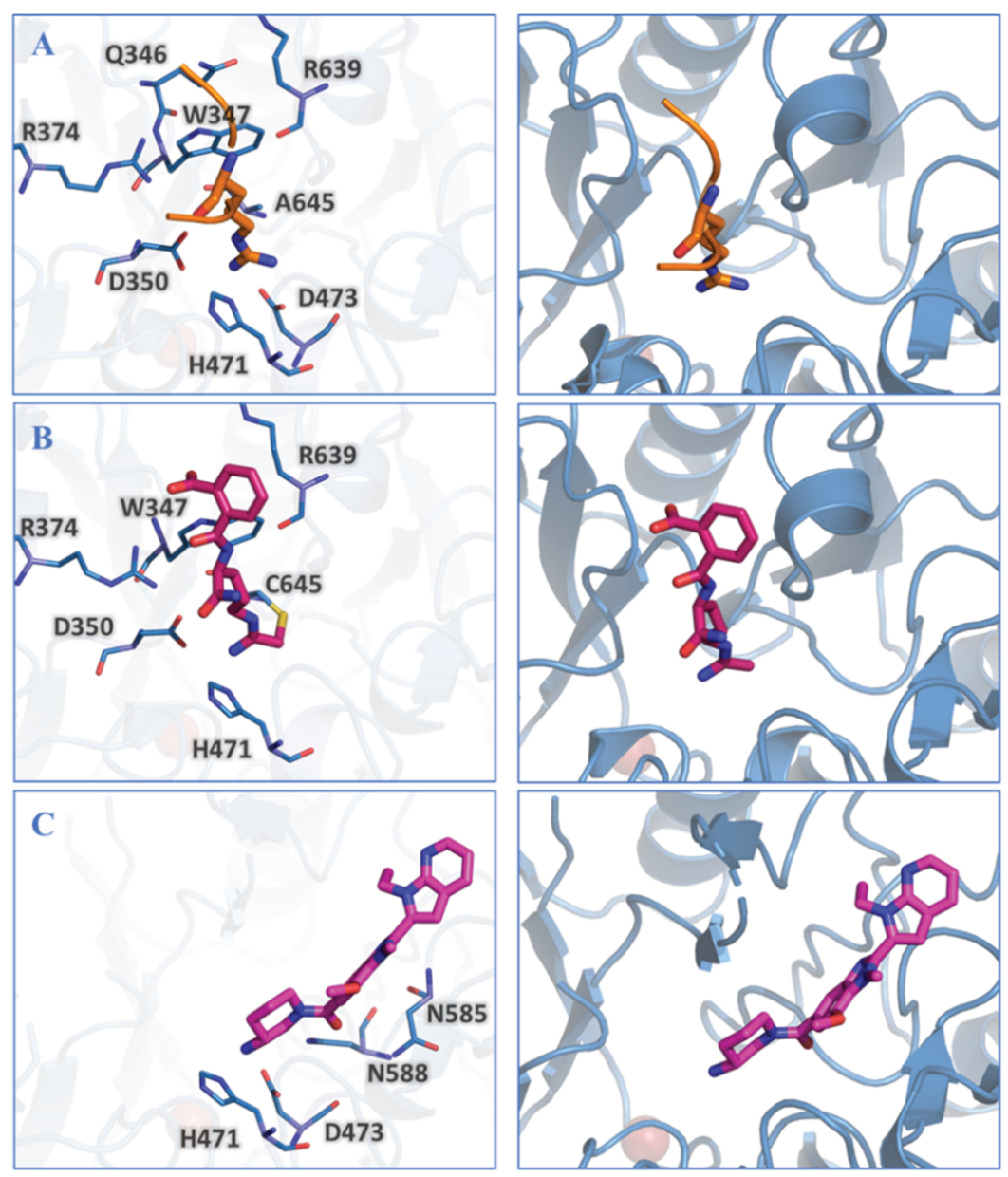

PDB: 2DEW (c645Amutation)

Substrate: histone tail

PDB: 3B1T

Inhibitor: 0 -Cl-amidine

Figure 5. Irreversible and reversible inhibitors of PAD4. Here the crystal structure of PAD4 is shown together with a histone arginine (natural substrate, orange), the irreversible inhibitor o-Cl-amidine (magenta) and the reversible inhibitor GSK199 (magenta). In the left panel, the PAD4 residues that interact with the substrate or inhibitor are highlighted. In the right panel, the position of the substrate/inhibitor within the crystal structure is shown. A. PAD4 interactions with arginine from a histone tail (sequence 6-TARKS-10). B. PAD4 interactions with irreversible inhibitor o-Cl-amidine, where the covalent interaction with $\mathrm{C645}$ is present. C. PAD4 interactions with reversible inhibitor GSK199. 


\subsection{Abbreviations}

PTM Post-translational modification

PAD Peptidyl arginine deiminase

NET Neutrophil extracellular trap

LTH Leukotoxic Hypercitrullination

ROS Reactive oxygen species

ENDS Elongated neutrophil-derived structures

DTT Dithiothreitol

RA Rheumatoid arthritis

DVT Deep vein thrombosis

TLRs Toll-like receptors

NADPH Dihydronicotinamide-adenine dinucleotide phosphate

$\mathrm{Cl}$-amidine $\quad \mathrm{N}$-alpha-benzoyl-N5-(2-chloro-1-iminoethyl)-L-ornithine amide

\subsection{Acknowledgements}

We thank China Scholarship Council for providing scholarship to author Xiaosong Liu. Tom Arfman is supported by a grant from the Dutch Thrombosis Society (TSN2019) 


\section{References}

1. Mann, M.; Jensen, O. N., Proteomic analysis of post-translational modifications. Nat Biotechnol 2003, 21 (3), 255-61.

2. Knuckley, B., et al., Protein arginine deiminase 4: evidence for a reverse protonation mechanism. Biochemistry 2007, 46 (22), 6578-87.

3. van Venrooij, W. J.; Pruijn, G. J., Citrullination: a small change for a protein with great consequences for rheumatoid arthritis. Arthritis Res 2000, 2 (4), 249-51.

4. Ordóñez, A., et al., Effect of citrullination on the function and conformation of antithrombin. FEBS Journal 2009, 276, 6763-6772.

5. Tarcsa, E., et al., Protein Unfolding by Peptidylarginine Deiminase. Journal of Biological Chemistry 1996, 271, 30709-30716.

6. Lundberg, K., et al., Citrullinated proteins have increased immunogenicity and arthritogenicity and their presence in arthritic joints correlates with disease severity. Arthritis Research \& Therapy 2005, 7, R458.

7. Vossenaar, E. R., et al., PAD, a growing family of citrullinating enzymes: genes, features and involvement in disease. Bioessays 2003, 25 (11), 1106-18.

8. Nakashima, K., et al., Molecular characterization of peptidylarginine deiminase in HL-60 cells induced by retinoic acid and 1alpha,25-dihydroxyvitamin D(3). The Journal of biological chemistry 1999, 274, 2778627792.

9. Nakashima, K., et al., Nuclear localization of peptidylarginine deiminase $\mathrm{V}$ and histone deimination in granulocytes. Journal of Biological Chemistry 2002, 277, 49562-49568.

10. Chang, X.; Han, J., Expression of peptidylarginine deiminase type 4 (PAD4) in various tumors. Molecular Carcinogenesis 2006, 45, 183-196.

11. Wang, Y., et al., Histone hypercitrullination mediates chromatin decondensation and neutrophil extracellular trap formation. The Journal of cell biology 2009, 184 (2), 205-213.

12. Stadler, S. C., et al., Dysregulation of PAD4-mediated citrullination of nuclear GSK3 $\beta$ activates TGF- $\beta$ signaling and induces epithelialto-mesenchymal transition in breast cancer cells. Proceedings of the National Academy of Sciences of the United States of America 2013, 110, 11851-11856.

13. Lee, Y. H., et al., Regulation of coactivator complex assembly and function by protein arginine methylation and demethylimination. Proceedings of the National Academy of Sciences of the United States of America 2005, 102, 3611-3616.

14. Guo, Q.; Fast, W., Citrullination of Inhibitor of Growth 4 (ING4) by Peptidylarginine Deminase 4 (PAD4) disrupts the interaction between ING4 and p53. Journal of Biological Chemistry 2011, 286, 17069-17078.

15. Guo, Q., et al., Discovery of peptidylarginine deiminase-4 substrates by protein array: Antagonistic citrullination and methylation of human ribosomal protein S2. Molecular BioSystems 2011, 7, 2286-2295.

16. Sorvillo, N., et al., Plasma peptidylarginine deiminase IV promotes VWF-platelet string formation and accelerates thrombosis after vessel injury. Circulation Research 2019, 125, 507-519.

17. Tanikawa, C., et al., Citrullination of RGG Motifs in FET Proteins by PAD4 Regulates Protein Aggregation and ALS Susceptibility. Cell Reports 2018, 22, 1473-1483.

18. Rohrbach, A. S., et al., Activation of PAD4 in NET formation. Front Immunol 2012, 3, 360.

19. Arita, K., et al., Structural basis for histone N-terminal recognition by human peptidylarginine deiminase 4 . Proceedings of the National Academy of Sciences of the United States of America 2006, 103, 5291-5296.

20. Brinkmann, V., Neutrophil Extracellular Traps Kill Bacteria. Science 2004, 303, 1532-1535.

21. Euler, M.; Hoffmann, M. H., The double-edged role of neutrophil extracellular traps in inflammation. Biochem Soc Trans 2019, 47 (6), 1921-1930.

22. Kaplan, M. J.; Radic, M., Neutrophil extracellular traps: double-edged swords of innate immunity. $J$ Immunol 2012, 189 (6), 2689-95

23. Konig, M. F.; Andrade, F., A Critical Reappraisal of Neutrophil Extracellular Traps and NETosis Mimics Based on Differential Requirements for Protein Citrullination. Front Immunol 2016, 7, 461.

24. Kenny, E. F., et al., Diverse stimuli engage different neutrophil extracellular trap pathways. Elife $2017,6$.

25. Fuchs, T. A., et al., Extracellular DNA traps promote thrombosis. Proceedings of the National Academy of Sciences of the United States of America 2010, 107, 15880-15885.

26. Perdomo, J., et al., Neutrophil activation and NETosis are the major drivers of thrombosis in heparininduced thrombocytopenia. Nature Communications 2019, 10, 1322.

27. Suzuki, A., et al., Decreased severity of experimental autoimmune arthritis in peptidylarginine deiminase type 4 knockout mice. BMC Musculoskeletal Disorders 2016, 17, 205.

28. Yokoyama, Y., et al., Circulating histone H3 levels in septic patients are associated with coagulopathy, multiple organ failure, and death: a single-center observational study. Thrombosis Journal 2019, $17,1$.

29. Biron, B. M., et al., PAD4 Deficiency Leads to Decreased Organ Dysfunction and Improved Survival in a Dual Insult Model of Hemorrhagic Shock and Sepsis. J Immunol 2018, 200 (5), 1817-1828. 
30. Arita, K., et al., Structural basis for Ca2+-induced activation of human PAD4. Nature Structural and Molecular Biology 2004, 11, 777-783.

31. Barclay, A. N., Ig-like domains: evolution from simple interaction molecules to sophisticated antigen recognition. Proc Natl Acad Sci U S A 1999, 96 (26), 14672-4.

32. Liu, Y.-L., et al., Probing the Roles of Calcium-Binding Sites during the Folding of Human Peptidylarginine Deiminase 4. Scientific Reports 2017, 7, 2429.

33. Krieger, E.; Vriend, G., YASARA View - molecular graphics for all devices - from smartphones to workstations. Bioinformatics (Oxford, England) 2014, 30, 2981-2982.

34. Liu, Y. L., et al., Functional role of dimerization of human peptidylarginine deiminase 4 (PAD4). PLoS One 2011, 6 (6), e21314.

35. Teo, C. Y., et al., Discovery of a new class of inhibitors for the protein arginine deiminase type 4 (PAD4) by structure-based virtual screening. BMC Bioinformatics 2012, 13 Suppl 17, S4.

36. Kingsley, L. J.; Lill, M. A., Substrate tunnels in enzymes: structure-function relationships and computational methodology. Proteins 2015, 83 (4), 599-611.

37. Holmes, C. L., et al., Insight into neutrophil extracellular traps through systematic evaluation of citrullination and peptidylarginine deiminases. Journal of Immunology Research 2019, 2019.

38. Robertson, W. G., et al., Ionized calcium in body fluids. Critical Reviews in Clinical Laboratory Sciences 1981, 15, 85-125.

39. Darrah, E., et al., Erosive rheumatoid arthritis is associated with antibodies that activate PAD4 by increasing calcium sensitivity. Science Translational Medicine 2013, 5.

40. Zhou, Y., et al., Perspective on Protein Arginine Deiminase Activity-Bicarbonate Is a pH-Independent Regulator of Citrullination. Frontiers in Immunology 2018, 9, 34.

41. Connell, E., Tietz Textbook of Clinical Chemistry and Molecular Diagnostics (5th edn). Annals of Clinical Biochemistry: International Journal of Laboratory Medicine 2012, 49, 615-615.

42. Terakawa, H., et al., Three types of mouse peptidylarginine deiminase: characterization and tissue distribution. Journal of biochemistry 1991, 110, 661-666.

43. Damgaard, D., et al., Reactive oxygen species inhibit catalytic activity of peptidylarginine deiminase. Journal of Enzyme Inhibition and Medicinal Chemistry 2017, 32, 1203-1208.

44. Damgaard, D., et al., Reduced glutathione as a physiological co-activator in the activation of peptidylarginine deiminase. Arthritis Res Ther 2016, 18 (1), 102.

45. Nagar, M., et al., Thioredoxin Modulates Protein Arginine Deiminase 4 (PAD4)-Catalyzed Citrullination. Frontiers in Immunology 2019, 10, 244.

46. Slack, J. L., et al., Autodeimination of protein arginine deiminase 4 alters protein-protein interactions but not activity. Biochemistry 2011, 50, 3997-4010.

47. Liu, X., et al., Autocitrullination of PAD4 does not alter its enzymatic activity: in vitro and in silico studies. The International Journal of Biochemistry \& Cell Biology 2021, 134, 105938.

48. Nathan, C., Neutrophils and immunity: challenges and opportunities. Nature Reviews Immunology 2006, 6, 173-182.

49. Segal, A. W., How Neutrophils Kill Microbes. Annual Review of Immunology 2005, 23, 197-223.

50. $\mathrm{Li}, \mathrm{P}$., et al., PAD4 is essential for antibacterial innate immunity mediated by neutrophil extracellular traps. Journal of Experimental Medicine 2010, 207, 1853-1862.

51. Thiama, H. R., et al., NETosis proceeds by cytoskeleton and endomembrane disassembly and PAD4mediated chromatin decondensation and nuclear envelope rupture. Proceedings of the National Academy of Sciences of the United States of America 2020, 117, 7326-7337.

52. Fuchs, T. A., et al., Novel cell death program leads to neutrophil extracellular traps. J Cell Biol 2007, 176 (2), 231-41.

53. Hirschfeld, J., et al., Modulation of Neutrophil Extracellular Trap and Reactive Oxygen Species Release by Periodontal Bacteria. Infect Immun 2017, 85 (12).

54. Tatsiy, O.; McDonald, P. P., Physiological Stimuli Induce PAD4-Dependent, ROS-Independent NETosis, With Early and Late Events Controlled by Discrete Signaling Pathways. Front Immunol 2018, 9, 2036.

55. Leshner, M., et al., PAD4 mediated histone hypercitrullination induces heterochromatin decondensation and chromatin unfolding to form neutrophil extracellular trap-like structures. Frontiers in immunology 2012, 3, 307-307.

56. Neeli, I., et al., Histone Deimination As a Response to Inflammatory Stimuli in Neutrophils. The Journal of Immunology 2008, 180, 1895-1902.

57. Saha, P., et al., PAD4-dependent NETs generation are indispensable for intestinal clearance of Citrobacter rodentium. Mucosal Immunology 2019, 12, 761-771.

58. Lewis, H. D., et al., Inhibition of PAD4 activity is sufficient to disrupt mouse and human NET formation. Nat Chem Biol. 2015, 11, 189-191.

59. Leffler, J., et al., Neutrophil extracellular traps that are not degraded in systemic lupus erythematosus activate complement exacerbating the disease. J Immunol 2012, 188 (7), 3522-31. 
60. Tillack, K., et al., T lymphocyte priming by neutrophil extracellular traps links innate and adaptive immune responses. J Immunol 2012, 188 (7), 3150-9.

61. Kumar, S. V. R., et al., Neutrophil extracellular trap-related extracellular histones cause vascular necrosis in severe GN. Journal of the American Society of Nephrology 2015, 26, 2399-2413.

62. Fuchs, T. A., et al., Histones induce rapid and profound thrombocytopenia in mice. Blood 2011, 118, 37083714.

63. Thalin, C., et al., Neutrophil Extracellular Traps: Villains and Targets in Arterial, Venous, and CancerAssociated Thrombosis. Arterioscler Thromb Vasc Biol 2019, 39 (9), 1724-1738.

64. Fousert, E., et al., Neutrophil Extracellular Traps (NETs) Take the Central Stage in Driving Autoimmune Responses. Cells 2020, 9 (4).

65. Silman, A. J.; Pearson, J. E., Epidemiology and genetics of rheumatoid arthritis. Arthritis Research 2002, 4, S265.

66. Fujii, K., et al., Rheumatoid arthritis: a synovial disease? Ann Rheum Dis 1999, 58 (12), 727-30.

67. Fisher, B. A.; Venables, P. J., Inhibiting citrullination in rheumatoid arthritis: taking fuel from the fire. Arthritis Res Ther 2012, 14 (1), 108.

68. Suzuki, A., et al., Functional haplotypes of PADI4, encoding citrullinating enzyme peptidylarginine deiminase 4, are associated with rheumatoid arthritis. Nature Genetics 2003, 34, 395-402.

69. Hua, J.; Huang, W., Peptidylarginine deiminase $4-104 \mathrm{C} / \mathrm{T}$ polymorphism and risk of rheumatoid arthritis: A pooled analysis based on different populations. PLoS One 2018, 13 (3), e0193674.

70. Tilvawala, R., et al., The Rheumatoid Arthritis-Associated Citrullinome. Cell Chemical Biology 2018, 25, 691-704.e6.

71. Kuhn, K. A., Antibodies against citrullinated proteins enhance tissue injury in experimental autoimmune arthritis. Journal of Clinical Investigation 2006, 116, 961-973.

72. Seri, Y., et al., Peptidylarginine deiminase type 4 deficiency reduced arthritis severity in a glucose-6phosphate isomerase-induced arthritis model. Scientific Reports 2015, 5, 13041.

73. Vincent, C., et al., Immunoblotting detection of autoantibodies to human epidermis filaggrin: a new diagnostic test for rheumatoid arthritis. The Journal of rheumatology 1998, 25, 838-846.

74. Mewar, D., et al., Independent associations of anti-cyclic citrullinated peptide antibodies and rheumatoid factor with radiographic severity of rheumatoid arthritis. Arthritis Research and Therapy 2006, 8, R128.

75. van Venrooij, W. J., et al., Autoantibodies to citrullinated antigens in (early) rheumatoid arthritis. Autoimmun Rev 2006, 6 (1), 37-41.

76. Rudd, K. E., et al., Global, regional, and national sepsis incidence and mortality, 1990-2017: analysis for the Global Burden of Disease Study. The Lancet 2020, 395, 200-211.

77. Xu, J., et al., Extracellular histones are major mediators of death in sepsis. Nature Medicine 2009, 15, 1318-1321.

78. Dwivedi, D. J., et al., Prognostic utility and characterization of cell-free DNA in patients with severe sepsis. Critical Care 2012, 16, R151.

79. Rhodes, A., et al., Plasma DNA concentration as a predictor of mortality and sepsis in critically ill patients. Critical Care 2006, 10, R60.

80. Clark, S. R., et al., Platelet TLR4 activates neutrophil extracellular traps to ensnare bacteria in septic blood. Nature Medicine 2007, 13, 463-469.

81. Meng, W., et al., Depletion of neutrophil extracellular traps in vivo results in hypersusceptibility to polymicrobial sepsis in mice. Critical Care 2012, 16, R137.

82. Cheng, L., et al., Comparative efficacy of vasoactive medications in patients with septic shock: a network meta-analysis of randomized controlled trials. Critical Care 2019, 23, 168.

83. Wildhagen, K. C. A. A., et al., Extracellular histone $\mathrm{H} 3$ levels are inversely correlated with antithrombin levels and platelet counts and are associated with mortality in sepsis patients. Thrombosis Research 2015, 136, 542-547.

84. Claushuis, T. A. M., et al., Role of Peptidylarginine Deiminase 4 in Neutrophil Extracellular Trap Formation and Host Defense during Klebsiella pneumoniae-Induced Pneumonia-Derived Sepsis. Journal of immunology (Baltimore, Md. : 1950) 2018, 201, 1241-1252.

85. Wendelboe, A. M.; Raskob, G. E., Global Burden of Thrombosis: Epidemiologic Aspects. Circulation Research 2016, 118, 1340-1347.

86. Kimball, A. S., et al., The Emerging Role of NETs in Venous Thrombosis and Immunothrombosis. Front Immunol 2016, 7, 236.

87. Massberg, S., et al., Reciprocal coupling of coagulation and innate immunity via neutrophil serine proteases. Nature Medicine 2010, 16, 887-896.

88. Gould, T. J., et al., Neutrophil Extracellular Traps Promote Thrombin Generation Through PlateletDependent and Platelet-Independent Mechanisms. Arteriosclerosis, Thrombosis, and Vascular Biology 2014, 34, 1977-1984. 
89. Laridan, E., et al., Neutrophil Extracellular Traps in Arterial and Venous Thrombosis. Seminars in Thrombosis and Hemostasis 2019, 45, 086-093.

90. McDonald, B., et al., Platelets and neutrophil extracellular traps collaborate to promote intravascular coagulation during sepsis in mice. Blood 2017, 129 (10), 1357-1367.

91. Semeraro, F., et al., Extracellular histones promote thrombin generation through platelet-dependent mechanisms: involvement of platelet TLR2 and TLR4. Blood 2011, 118, 1952-1961.

92. Beiter, T., et al., Neutrophils release extracellular DNA traps in response to exercise. Journal of Applied Physiology 2014, 117, 325-333.

93. Morris, G., et al., Preventing the development of severe COVID-19 by modifying immunothrombosis. Life Sci 2021, 264, 118617.

94. Martinod, K., et al., Neutrophil histone modification by peptidylarginine deiminase 4 is critical for deep vein thrombosis in mice. Proceedings of the National Academy of Sciences of the United States of America 2013, 110, 8674-8679.

95. Gollomp, K., et al., Inhibition of Neutrophil Extracellular Trap Release through the Disruption of PAD4 Leads to Decreased Neutrophil and Platelet Accumulation at Sites of Venular Vascular Injury in a Murine Model of Heparin-Induced Thrombocytopenia. Blood 2017, 130, 2268-2268.

96. Thompson, P. R., Citrullination Inhibits SERPIN Activity. The FASEB Journal 32, 104.3-104.3.

97. Ordóñez, A., et al., Increased levels of citrullinated antithrombin in plasma of patients with rheumatoid arthritis and colorectal adenocarcinoma determined by a newly developed ELISA using a specific monoclonal antibody. Thrombosis and Haemostasis 2010, 104, 1143-1149.

98. Stone, E. M., et al., Inactivation of two diverse enzymes in the amidinotransferase superfamily by 2chloroacetamidine: Dimethylargininase and peptidylarginine deiminase. Biochemistry 2005, 44, 1374413752.

99. Nemmara, V. V.; Thompson, P. R., Development of Activity-Based Proteomic Probes for Protein Citrullination. Curr Top Microbiol Immunol 2019, 420, 233-251.

100. Cheng, O. Z.; Palaniyar, N., NET balancing: a problem in inflammatory lung diseases. Front Immunol 2013, $4,1$. 



\section{Chapter}

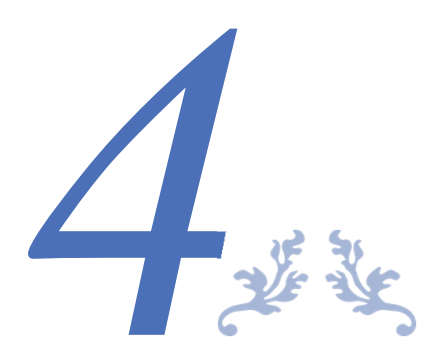

\section{Autocitrullination of PAD4 does not alter its enzymatic activity: in vitro and in silico studies}

Xiaosong Liu ${ }^{1}$, Kanin Wichapong ${ }^{1}$, Sebastiaan Lamers ${ }^{1}$, Chris P. M. Reutelingsperger ${ }^{1}$, Gerry A. F. Nicolaes ${ }^{1}$

1Department of Biochemistry, Cardiovascular Research Institute Maastricht (CARIM), Maastricht University, Maastricht, The Netherlands 


\section{Abstract}

Background Protein arginine deiminase 4 (PAD4) is an enzyme capable of converting arginine (positively charged residue) into citrulline (neutral residue). PAD4 is a promiscuous enzyme since it citrullinates various substrates, including small peptides, large proteins and itself. The effect of autocitrullination on PAD4 activity remains controversial and inconclusive. We hypothesized that PAD4 autocitrullination may influence the activity of PAD4 by indirectly altering its binding to substrate.

Methods We employed mass spectrometry analysis to study the process of autocitrullination. The kinetics of citrullination of PAD4 and citrullinated PAD4 (citPAD4) towards substrates of different sizes (0.17-15.4 kDa), i,e free arginine, a peptidyl substrate, and histone $\mathrm{H} 3$, were studied by colorimetric assay and Western blotting. Molecular dynamics (MD) simulations were performed to investigate structural dynamic and binding properties of PAD4/citPAD4 in the absence and presence of substrates.

Results We observed that 23/27 arginine residues in PAD4 (85\%) can be citrullinated, including R372, R374 and R639 located near the substrate binding pocket. PAD4 and citPAD4 expressed comparable enzymatic activities towards different substrates. In agreement with experimental results, MD simulations indicated that autocitrullination does not change the shape of the substrate binding pocket and PAD4/citPAD4 exhibited comparable binding free energy with a H3-derived peptidyl substrate (6TARKS-10).

Conclusion While the effect of autocitrullination on PAD4 activity thus far remained unclear and controversial, here we have demonstrated that autocitrullination does not affect the activity of PAD4. Thus, the regulation of PAD4 activity is probably not controlled by autocitrullination but likely by other mechanisms that need further investigation.

Keywords: PAD4, autocitrullination, citrullination, PAD4 activity, histone $\mathrm{H} 3$, protein-protein interactions (PPIs)

\section{Graphical Abstract}

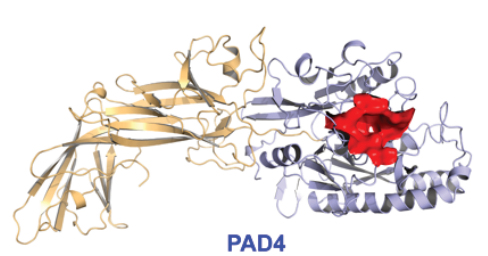

In vitro

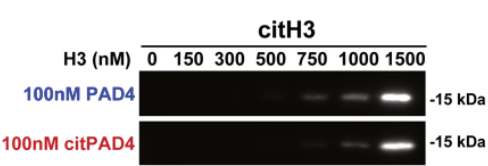

$\mathrm{Ca}^{2+} \downarrow 2 \mathrm{~h}$ incubation
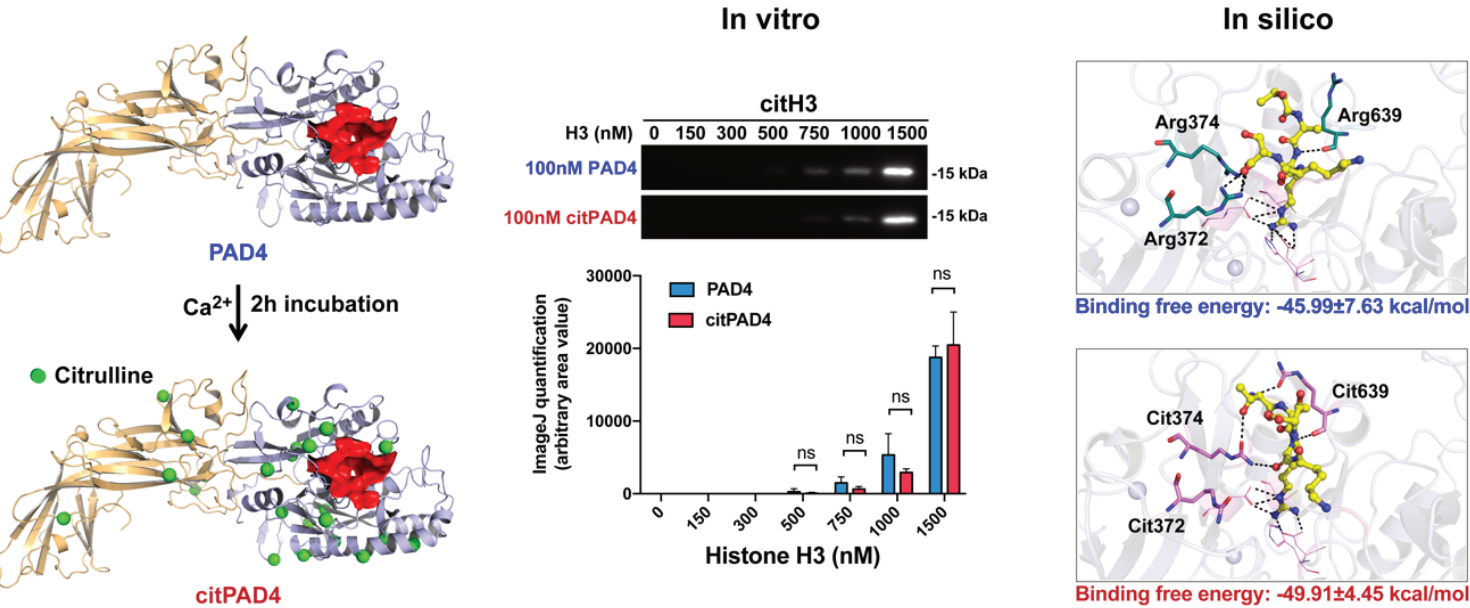


\subsection{Introduction}

The peptidyl arginine deiminases (PAD) form a family of proteins that catalyse the process of citrullination, during which an arginine residue is converted into a citrulline. The human PAD family contains five family members: PAD1-4, and PAD6. By removing the positive charge from arginine residues, citrullination can alter protein-protein interactions (PPIs) which can consequently affect the function of a protein. PAD4 is the only member of the PAD family that carries a nuclear localization signal sequence 1 . Through this sequence, PAD4 is able to translocate to the cell nucleus and perform a number of tasks, among which the citrullination of nuclear histones. PAD4-catalyzed citrullination of histones is regarded an essential trigger for the formation of neutrophil extracellular traps (NETs), neutrophil structures which are linked to a variety of pathological conditions ${ }^{2}$. PAD4 has been implicated in several human diseases such as rheumatoid arthritis ${ }^{3}$, cancer ${ }^{4}$, NET-related diseases (e.g. deep vein thrombosis (DVT) ${ }^{5}$ and inflammatory diseases ${ }^{6}$. Hence, PAD4 is receiving an increasing interest as it represents a potential target for pharmacological intervention in the treatment of different diseases. Even though the structure-function relationship of PAD4, and its implications in human disease are gradually being uncovered, the underlying mechanisms for the regulation of PAD4 activity under physiological conditions are incompletely understood.

PAD4 can citrullinate many proteins, peptide-based substrates and small molecule arginine mimics ${ }^{7}$. Histones, located in the cell nucleus, are among the physiological substrates of PAD4 ${ }^{8}$. PAD4 can citrullinate various substrates without a clear specificity other than a preference for an RGG motif in its substrates $^{9}$, which makes it a rather promiscuous enzyme. Importantly, PAD4 expresses autocitrullination activity ${ }^{10,11}$. However, whether autocitrullination influences the activity of PAD4, which would thereby represent an autoregulatory process, is still unclear. Over the last decades, only two studies investigating autocitrullination of PAD4 were performed and contradicting findings were reported. On the one hand, Andrade and coworkers ${ }^{10}$ identified many citrulline sites in PAD4, including at R372 and R374 located at the binding pocket, and concluded that PAD4 lost its activity completely after autocitrullination, using HL-60 cell lysate as PAD4 substrate. On the other hand, the group of Thompson ${ }^{11}$ demonstrated that PAD4 autocitrullination at R372, R374, and R639 did not decrease PAD4 activity towards both small substrates (e.g. N-a-benzoyl L-arginine ethyl ester (BAEE)) and big substrates (e.g. histones $\mathrm{H} 3$ and $\mathrm{H} 4$ ). Instead, they reported that PAD4 autocitrullination weakened protein-protein interactions between PAD4 and its binding partners, including citrullinated histone $\mathrm{H} 3$, histone deacetylase 1 (HDAC1) and protein arginine methyltransferase 1 (PRMT1). Given the apparent incomplete description of PAD4 autocitrullination, we hypothesized that autocitrullination of PAD4 could indirectly change substrate binding affinity, by changing the electrostatic surface potential, which may or may not affect substrate binding and catalytic efficiency, and represent a potential mechanism of PAD4 for regulation of its physiological activity.

In this study, we probed the autocitrullination of PAD4 in a time- and concentration-dependent manner in in vitro systems composed of purified components. The effects of autocitrullination on PAD4 activity were next evaluated by use of three different substrates, i.e.: free arginine $(0.17 \mathrm{kDa})$, a synthetically 
labeled tripeptide $(0.503 \mathrm{kDa})$ and a full-length protein, histone H3 $(15.4 \mathrm{kDa})$. To further analyze potential effects on the structural stability and substrate binding we compared citrullinated PAD4 and non-citrullinated PAD4 by various in silico methods, (i.e. molecular docking and molecular dynamics (MD) simulations coupled with binding free energy (BFE) calculations).

\subsection{Material and methods}

\subsubsection{PAD4 expression and purification}

A PAD4 expression vector containing an N-terminal Strep-Tag II (WSHPQFEK), was cloned into the pET-51b(+) plasmid (Novagen, Darmstadt, Germany). The PAD4 expression construct was transformed into E. coli BL21(DE3)pLysS competent cells (Novagen) for expression. PAD4 expression was induced by addition of $1 \mathrm{mM}$ IPTG, followed by a 4-hour incubation under gentle agitation at $23^{\circ} \mathrm{C}$. Bacterial pellets were resuspended in lysate buffer $(100 \mathrm{mM}$ Tris-HCl, $150 \mathrm{mM} \mathrm{NaCl}, 1 \mathrm{mM}$ EDTA, and $0.5 \mathrm{mM}$ TCEP) and lysed by sonication. After centrifugation at $9400 \mathrm{xg}$ for $30 \mathrm{~min}$ at $4{ }^{\circ} \mathrm{C}$, the supernatant was collected and loaded onto a Strep-Tactin column (IBA, Germany) which was equilibrated with wash buffer (WB, $100 \mathrm{mM}$ Tris-HCl, PH 8.0, $150 \mathrm{mM} \mathrm{NaCl}, 1 \mathrm{mM}$ EDTA). During loading, $2 \mathrm{nM}$ biotin-blocker was added to prevent unspecific biotin from binding to the column. The purification was performed under gravity flow. PAD4 was eluted from the column by addition of elution buffer (EB, $100 \mathrm{mM}$ Tris- $\mathrm{HCl}, \mathrm{pH}$ $8.0,150 \mathrm{mM} \mathrm{NaCl}, 1 \mathrm{mM}$ EDTA, $2.5 \mathrm{mM}$ desthiobiotin). Note that during the purification the chelator EDTA was present to prevent the calcium induced activation of PAD4. Before storage a buffer switch to storage buffer (Tris- $\mathrm{HCl}, \mathrm{pH}$ 8.0, $150 \mathrm{mM} \mathrm{NaCl}, 1 \mathrm{mM}$ EDTA, $1 \mathrm{mM}$ TCEP) was performed by use of a disposable PD10 desalting column (GE Healthcare). The purity of PAD4 was verified by SDS-PAGE and Coomassie Brilliant blue staining (Bio-Rad, Hemel Hempstead, UK). Aliquots were snap-frozen and stored in storage buffer containing at $-80^{\circ} \mathrm{C}$ until further use.

\subsubsection{Western blot analysis of PAD4 autocitrullination}

PAD4 (0-1000 nM) was incubated in activation buffer (100 mM Tris- $\mathrm{HCl}, \mathrm{PH} 7.6,150 \mathrm{mM} \mathrm{NaCl}, 10 \mathrm{mM}$ $\mathrm{CaCl}_{2}, 1 \mathrm{mM} \mathrm{TCEP}$ ) at $37^{\circ} \mathrm{C}$ for $1 \mathrm{~h}$ or $2 \mathrm{~h}$. The reaction was quenched by addition of standard gel sample buffer containing $1.25 \%$ beta-mercaptoethanol and heated for 10 minutes at $95^{\circ} \mathrm{C}$. Subsequently, samples were run on $15 \%$ SDS-polyacrylamide gels and transferred to PVDF membranes (Bio-rad). Next, citrulline was chemically modified by 2,3-butanedione monoxime and antipyrine in a strong acidic $(\mathrm{pH}<1)$ environment and detected by anti-citrulline (modified) monoclonal antibody (MABS487, SigmaAldrich, St. Louis, MO, USA) as was described ${ }^{12}$ in combination with a secondary rabbit anti-human IgG-HRP conjugated antibody (AP101P, Sigma-Aldrich. Membranes were imaged after incubation with ECL Western Blotting substrate (ab65623, Abcam, Cambridge, UK) to quantitate the citrullinated protein signal using ImageQuant TL software (GE Healthcare, Little Chalfont, UK). 


\subsubsection{Mass spectrometry (MS) analysis of autocitrullination sites in PAD4}

MS samples were prepared by transfer of $1 \mu \mathrm{M}$ PAD4 into activation buffer (100 mM Tris-HCl, pH 7.6, $150 \mathrm{mM} \mathrm{NaCl}, 10 \mathrm{mM} \mathrm{CaCl}_{2}, 1 \mathrm{mM}$ TCEP) at $37^{\circ} \mathrm{C}$ for $0,30,60$ and 120 minutes. The reactions were quenched by addition of standard gel sample buffer containing $1.25 \%$ beta-mercaptoethanol and heated for 10 minutes at $95^{\circ} \mathrm{C}$. PAD4 was detected after $15 \%$ SDS-PAGE and Coomassie blue staining. PAD4 protein bands of $75 \mathrm{kDa}$ were excised from the gel and digested on a MassPREP digestion robot (Waters, Manchester UK) with trypsin or chymotrypsin following an earlier described protocol ${ }^{13}$. Digested peptides were then loaded on a nanoflow HPLC instrument (Dionex ultimate 3000, Thermo Scientific) for peptide separation ${ }^{14}$. The MS data were acquired with a Q Exactive HPLC coupled mass spectrometer (Thermo Scientific) with a nanoelectrospray Flex ion source (Proxeon, Thermo Scientific) ${ }^{14}$. In combination with Thermo proteome Discoverer 2.2.0 software, a sequence search against the UniProt human protein database was used to identify peptides ${ }^{14}$. Cut-off criteria for citrulline detection used ${ }^{13}$ were a minimum intensity of $1.6^{*} 10^{5}$ ions/s for a peptide/mass peak to initiate a data dependent scan for further database search. The minimum signal-to-noise $(\mathrm{S} / \mathrm{N})$ threshold was 1.5 and the under-fill ratio was defined as $1.0 \%$. UniProt database thresholds settings for detecting citrullinated peptide/peaks are $10 \mathrm{ppm}$ for the precursor mass and 0.02 Da for the fragment mass. Citrulline residues were identified as a mass increase of $0.984 \mathrm{Da}$ as compared to arginine residues. An identified arginine that did not reach the cut-off for citrulline was defined as non- citrullinated while an arginine that exceeded the cutoff was defined as citrullinated. When an arginine was present in a fragment that could not be detected by MS (when fragments were too small or too big) it was defined as "not detected".

\subsubsection{Preparation of autocitrullinated PAD4 (citPAD4)}

As inferred from preliminary MS results, we observed a maximal PAD4 autocitrullination after incubation of PAD4 at a concentration of $1 \mu \mathrm{M}$ for 2 hours. These conditions resulted in a PAD4 preparation which we describe as citPAD4. Thus, to prepare citPAD4, PAD4 $(1 \mu \mathrm{M})$ was pre-incubated in activation buffer (100 mM Tris- $\mathrm{HCl}, \mathrm{PH} 7.6,150 \mathrm{mM} \mathrm{NaCl}, 10 \mathrm{mM} \mathrm{CaCl}, 1 \mathrm{mM} \mathrm{TCEP}$ ) for 2 hours at $37^{\circ} \mathrm{C}$. The citPAD4 was then immediately diluted to a concentration of $400 \mathrm{nM}$ and $100 \mathrm{nM}$ in buffer $(100 \mathrm{mM}$ Tris- $\mathrm{HCl}, \mathrm{pH}$ 7.6, $150 \mathrm{mM} \mathrm{NaCl}, 1 \mathrm{mM}$ TCEP). Aliquots were freshly used to investigate the enzymatic activity of citPAD4.

\subsubsection{Probing the enzymatic properties of PAD4 and citPAD4}

PAD4 activity was determined by use of the so-called COLDER assay (Color Developed assay) ${ }^{15}$, a discontinuous colorimetric assay that directly measures the amount of citrulline formed from arginine. We aimed to probe the activity of PAD4 and citPAD4 towards various substrates, ranging from small molecules to large proteins, and to this end we selected three distinctly different substrates: free arginine $(0.174 \mathrm{kDa})$, a tripeptidic chromogenic substrate $(0.503 \mathrm{kDa})$, and histone $\mathrm{H} 3$, a $15.4 \mathrm{kDa}$ protein.

To probe the activity towards free arginine, free arginine (0-30 $\mathrm{mM})$ in activation buffer was preincubated for 10 minutes at $37^{\circ} \mathrm{C}$, after which $400 \mathrm{nM}$ PAD4/citPAD4 was added to start the reaction. 
Reactions were quenched after exactly 10 minutes by addition of $200 \mu \mathrm{L}$ of freshly prepared COLDER solution consisting of solutions $A$ and $B$ in a ratio of 1:3 (solution $A: 80 \mathrm{mM}$ diacetyl monoxime, $3 \mathrm{mM}$ antipyrine, solution $\left.\mathrm{B}: 3 \mathrm{M} \mathrm{H}_{3} \mathrm{PO}_{4}, 6 \mathrm{M} \mathrm{H}_{2} \mathrm{SO}_{4}, 2 \mathrm{mM} \mathrm{NH}_{4} \mathrm{Fe}\left(\mathrm{SO}_{4}\right)_{2}\right)$. Next, the reaction volume was incubated for $30 \mathrm{~min}$ at $95^{\circ} \mathrm{C}$ to allow color development. Absorbance was then measured at $450 \mathrm{~nm}^{16}$,

17 in a microplate reader (BioTek, France). The amount of citrulline generated during the reaction was determined by conversion of absorbance values based on a citrulline standard curve (Figure S1). Blank measurements were included where the substrate was replaced by activation buffer only and were $<2 \%$ of final OD reading. The amount of citrulline contained by citPAD4 was measured in triplicates after subtraction of assay blanks and averages were calculated.

For the peptidic substrate (CS-21(66) (Hyphen Biomed, France), having a sequence of p-Glu-Pro-Arg$\mathrm{pNa}, 2 \mathrm{mM}$ of peptide was incubated with activation buffer for 10 minutes at $37^{\circ} \mathrm{C}$, after which $400 \mathrm{nM}$ of PAD4/citPAD4 was added to start the reaction. Reactions were quenched after varying incubation times ( $0-180$ minutes) by addition of $200 \mu \mathrm{L}$ of freshly prepared COLDER solution. Similar protocols as described above were employed to determine the amount of citrulline generated.

To determine the citrullination of the protein substrate (histone $\mathrm{H} 3$ ), we adjusted the experimental protocols so as to find the optimal reaction conditions for histone $\mathrm{H} 3$ citrullination. By variation of the enzyme (0-1000 $\mathrm{nM})$ and incubation time (0-60 $\mathrm{min})$, we determined that use of $100 \mathrm{nM}$ PAD4 for 10 minutes is optimal for histone $\mathrm{H} 3$ citrullination (Figure S2). Next, to investigate the enzymatic activity of PAD4/citPAD4 towards histone H3, histone H3 (calf thymus histone H3, 11034758001, Roche, Basel, Switzerland), was incubated at varying concentrations $(0-1500 \mathrm{nM})$ for 10 minutes at $37^{\circ} \mathrm{C}$ in activation buffer, followed by addition of $100 \mathrm{nM}$ PAD4/citPAD4 to initiate citrullination for exactly 10 minutes. Reactions were next quenched by addition of gel sample buffer containing $1.25 \%$ mercaptoethanol and heated for 10 minutes at $95^{\circ} \mathrm{C}$. The citrullinated histone $\mathrm{H} 3$ signal was detected by means of an anticitrulline monoclonal antibody, as described above for PAD4 autocitrullination detection by Western blotting.

\subsubsection{Molecular dynamic (MD) simulations and Binding Free Energy (BFE) Calculations}

A complete structure of human PAD4 was constructed with the YASARA Homology Modelling module ${ }^{18}$ by addition of missing residues and loop structures to the PDB template structure 3B1U. The citPAD4 structure was built by in silico mutation and subsequent optimization of arginine residues (see Table 1) into citrullines in the generated PAD4 model.

Prior to running MD simulations, special force fields for citrulline residue were created using the PyRED server ${ }^{19}$. PAD4 or citPAD4 in complex with a histone H3-derived peptide substrate which we named $\mathrm{R} 8 \mathrm{H} 3$ (with sequence 6-TARKS-10 from the $\mathrm{N}$-terminus of histone $\mathrm{H} 3$ ) were subjected to MD simulation. Standard parameters (i.e. ff14SB force field, explicit TIP3P water models, temperature at $300 \mathrm{~K}$, and pressure at 1 bar) and similar steps and protocols (such as energy minimization, a position-restrained phase, and production MD run using time step of $2 \mathrm{fs}$ while applying SHAKE constraint) as previously 
described ${ }^{20,} 21$ were applied to perform MD simulations for all systems (apo forms and PAD4/citPAD4$\mathrm{R} 8 \mathrm{H} 3$ complexes) for $100 \mathrm{~ns}$. The derived MD snapshots were utilized to investigate structural and dynamic properties (e.g. root mean square deviation (RMSD), clustering analysis, principle component analysis (PCA) and free energy landscape (FEL)) and $\mathrm{H}$-bond interactions between PAD4/citPAD4 and substrate (RR8H3) by utilization of the CPPTRAJ module using standard parameters as described in the AMBER16 manual ${ }^{22}$.

For the PAD4/citPAD4-R8H3 complexes, BFEs were calculated by using the molecular mechanics/generalized Born surface area (MM/GBSA, GB model 2) method ${ }^{23}$. MD snapshots were extracted from the last $20 \mathrm{~ns}$ (100 frames) for relative BFE calculation by approximation of the BFE $\left(\Delta G_{\text {binding }}\right)$ from the enthalpy contributions $(\Delta H)$ only $\left(\Delta G_{\text {binding }} \sim \Delta H\right)$ as previously explained ${ }^{20}$. To analyze and identify key residues of PAD4/citPAD4 that interact with the substrate, per-residue energy decomposition (DC) was also calculated by using the same MM/GBSA method (GB model 2). Both BFE and DC analysis were computed using the MMPBSA.py module ${ }^{24}$ in AMBER16.

\subsection{Results}

\subsubsection{Identification of PAD4 autocitrullination by Western blot and Mass Spectrometry (MS)}

The Western blots as shown in Figure 1 indicate that autocitrullination of PAD4 increases over time in a concentration dependent manner. No significant difference can be observed between the $1 \mathrm{~h}$ and $2 \mathrm{~h}$ incubation timepoint.

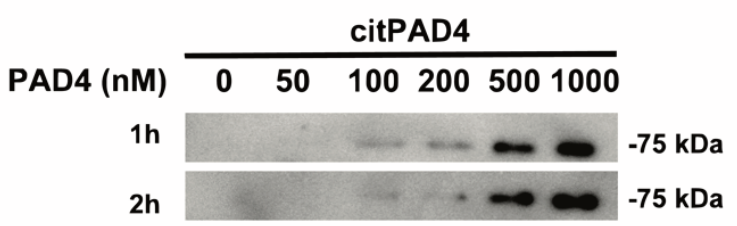

Figure 1. Autocitrullination of PAD4. PAD4 (0-1000 nM) was incubated in the presence of $10 \mathrm{mM}$ calcium at $37^{\circ} \mathrm{C}$ for $1 \mathrm{~h}$ or $2 \mathrm{~h}$. Equal amounts (75 $\mathrm{ng}$ ) of PAD4 were loaded in each of the panels (10-1000 nM) for SDS-PAGE analysis. The citrulline signal in western blot was visualized using chemiluminescence technique.

By utilization of MS, we identified $0,7,14$ and 23 citrulline residues at time points $0,30,60$, and 120 minutes, respectively (Table 1 and Figure 2). As observed in the Western blot experiment, autocitrullination of PAD4 occurred in a time-dependent manner. At 60 minutes of incubation, we observed that about half of all arginine residues (14/27) were citrullinated, with none of these citrullines located within $7 \AA$ distance to the catalytic pocket of PAD4 (Figure 2C). After 120 minutes of incubation (Figure 2D), 85\% of the arginine residues (23/27) were citrullinated, with the $3 / 4$ remaining arginines located in relatively inaccessible, buried positions. Importantly, citrullinations at R372, R374 and R639, located at the entrance of the catalytic pocket, were detected. These residues, R372, R374 and R639, are involved in binding and interaction with substrates or inhibitors as can be observed in experimental 
crystal structures ${ }^{25-28}$. Therefore, we hypothesized that when any of these three arginine residues (R372, R374 and R639) are citrullinated, this may affect PAD4 activity through changed substrate interactions. To address this hypothesis, we next investigated the effect of autocitrullination on PAD4 activity toward different substrates.

Table 1. Citrullination sites of PAD4 observed within 0-120 minutes. " $\checkmark$ " indicates identified citrullinated arginine sites. " $X$ " indicates identified non-citrullinated arginine sites, " $N$ " indicates not detected arginine sites and unknown for citrullination. The proximate environment of arginine is indicated by the neighboring amino acids in the sequence column at the right.

\begin{tabular}{|c|c|c|c|c|c|c|}
\hline PAD4 & $\begin{array}{c}\text { ARG } \\
\text { Position }\end{array}$ & $\begin{array}{c}\text { Omin } \\
\text { incubation }\end{array}$ & $\begin{array}{c}30 \mathrm{~min} \\
\text { incubation }\end{array}$ & $\begin{array}{c}60 \min \\
\text { incubation }\end{array}$ & $\begin{array}{c}120 \min \\
\text { incubation }\end{array}$ & $\begin{array}{c}\text { Sequence } \\
\text { in PAD4 }\end{array}$ \\
\hline \multirow{9}{*}{$\begin{array}{l}\mathrm{N} \text {-terminal } \\
\text { domain }\end{array}$} & Arg8 & $\mathrm{N}$ & $\mathrm{N}$ & $\mathrm{N}$ & $\checkmark$ & ${ }^{6} \mathrm{LIRVT}^{10}$ \\
\hline & Arg123 & $x$ & $x$ & $x$ & $\checkmark$ & 121|TRTG125 \\
\hline & Arg131 & $\mathrm{N}$ & $N$ & $\mathrm{~N}$ & $\checkmark$ & ${ }^{129} \mathrm{PTRAV}{ }^{133}$ \\
\hline & Arg137 & $\mathrm{N}$ & $\mathrm{N}$ & $\mathrm{N}$ & $x$ & ${ }^{135} \mathrm{DQRTW}^{139}$ \\
\hline & Arg156 & $x$ & $x$ & $x$ & $x$ & ${ }^{154} \mathrm{CDRDN}^{158}$ \\
\hline & Arg205 & $x$ & $x$ & $\checkmark$ & $\checkmark$ & 203VARSE 207 \\
\hline & Arg212 & $\mathrm{N}$ & $\checkmark$ & $\checkmark$ & $\checkmark$ & ${ }^{210} \mathrm{KVRVF}^{214}$ \\
\hline & Arg218 & $x$ & $\checkmark$ & $\checkmark$ & $\checkmark$ & ${ }^{216}$ ATRGK ${ }^{220}$ \\
\hline & Arg292 & $\mathrm{N}$ & $\mathrm{N}$ & $\mathrm{N}$ & $\mathrm{N}$ & ${ }^{290}$ VFRVA ${ }^{294}$ \\
\hline \multirow{18}{*}{$\begin{array}{c}\text { C-terminal } \\
\text { domain }\end{array}$} & Arg372 & $x$ & $x$ & $x$ & $\checkmark$ & ${ }^{370}$ SPRNR $^{374}$ \\
\hline & Arg374 & $\mathrm{N}$ & $\mathrm{N}$ & $\mathrm{N}$ & $\checkmark$ & ${ }^{372}$ RNRGL 376 \\
\hline & Arg383 & $x$ & $\checkmark$ & $\checkmark$ & $\checkmark$ & ${ }^{381}$ IKRVM $^{385}$ \\
\hline & Arg394 & $x$ & $\checkmark$ & $\checkmark$ & $\checkmark$ & 392VTRGP396 \\
\hline & Arg419 & $x$ & $x$ & $\checkmark$ & $\checkmark$ & 417TVRGK ${ }^{421}$ \\
\hline & Arg427 & $x$ & $x$ & $\checkmark$ & $\checkmark$ & ${ }^{425}$ LGRIL $^{429}$ \\
\hline & Arg441 & $x$ & $\checkmark$ & $\checkmark$ & $\checkmark$ & ${ }^{439} \mathrm{DSRQM}^{443}$ \\
\hline & Arg484 & $x$ & $x$ & $x$ & $\checkmark$ & ${ }^{482} \mathrm{PDRKG}^{486}$ \\
\hline & Arg488 & $\mathrm{N}$ & $\mathrm{N}$ & $\checkmark$ & $\checkmark$ & ${ }^{486}$ GFRLL 490 \\
\hline & Arg495 & $x$ & $x$ & $\checkmark$ & $\checkmark$ & ${ }^{493} S P R S C 497$ \\
\hline & Arg536 & $\mathrm{N}$ & $\checkmark$ & $\checkmark$ & $\checkmark$ & ${ }^{534}$ TLREH $^{538}$ \\
\hline & Arg544 & $x$ & $\checkmark$ & $\checkmark$ & $\checkmark$ & 542VERCI546 \\
\hline & Arg550 & $x$ & $x$ & $x$ & $\checkmark$ & ${ }^{548} \mathrm{WNREL}^{552}$ \\
\hline & Arg555 & $x$ & $x$ & $\checkmark$ & $\checkmark$ & ${ }^{553}$ LKREL $^{557}$ \\
\hline & Arg609 & $\mathrm{N}$ & $\mathrm{N}$ & $\mathrm{N}$ & $\mathrm{N}$ & ${ }^{607}$ NGRCC 611 \\
\hline & Arg639 & $\mathrm{N}$ & $\mathrm{N}$ & $\mathrm{N}$ & $\checkmark$ & ${ }^{637} \mathrm{HIRHG}^{641}$ \\
\hline & Arg650 & $x$ & $x$ & $x$ & $\checkmark$ & ${ }^{648} \mathrm{NVRRK}{ }^{652}$ \\
\hline & Arg651 & $\mathrm{N}$ & $\mathrm{N}$ & $\checkmark$ & $\checkmark$ & ${ }^{649}$ VRRKP'53 \\
\hline counts & 27 & 0 & 7 & 14 & 23 & \\
\hline
\end{tabular}


A

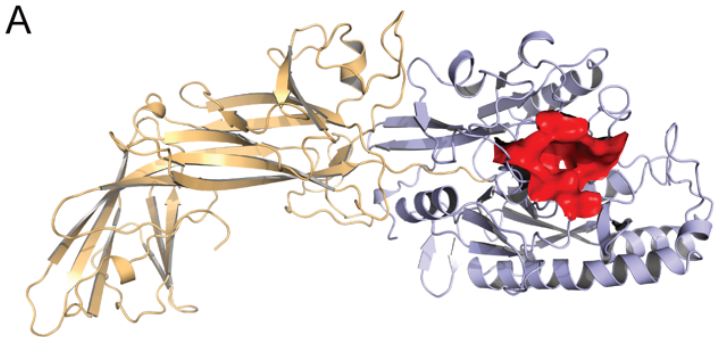

$$
t=0 \min
$$

C

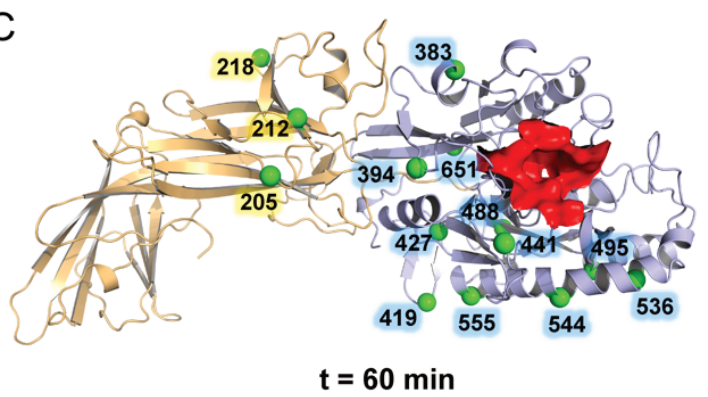

B

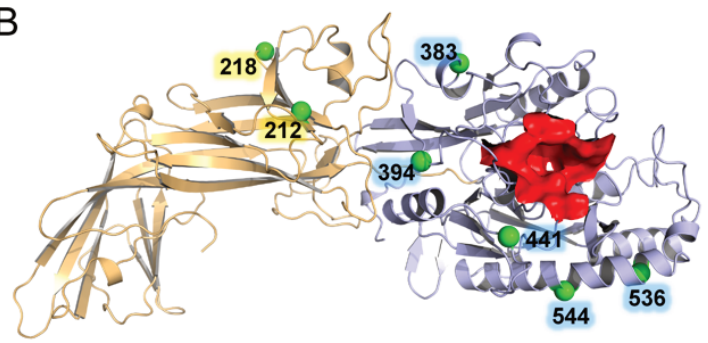

$\mathbf{t}=\mathbf{3 0} \min$

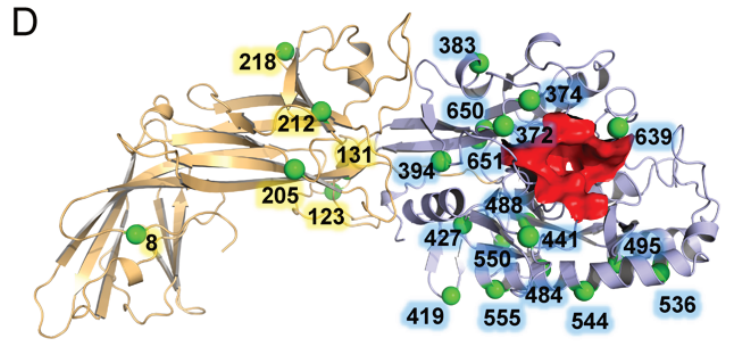

$t=120 \min$

Figure 2. Citrullination sites of PAD4 along incubation time. The PAD4 (1 $\mu M)$ was incubated for 0-120 minutes in the presence of $10 \mathrm{mM}$ calcium at $37^{\circ} \mathrm{C}$. The detected citrulline sites at $0 \mathrm{~min}(A), 30 \mathrm{~min}(B), 60 \mathrm{~min}(C), 120 \mathrm{~min}(D)$ are highlighted by green spheres. The lightorange part represents the $\mathrm{N}$-terminal domain and the lightblue part indicates the C-terminal domain. The catalytic binding pocket was highlighted by red surface.

\subsubsection{Effect of PAD4 autocitrullination on its activity towards substrates of varying size}

\subsubsection{Free Arginine substrate, $0.17 \mathrm{kDa}$}

PAD4 and citrullinated PAD4 (citPAD4) show enzymatic activity towards free arginine, characterized by calculated $\mathrm{K}_{\mathrm{m}}$ values of $8.35 \pm 1.25 \mathrm{mM}$ and $7.40 \pm 2.22 \mathrm{mM}(\mathrm{p}=0.0685)$, respectively (Figure 3A). The

A

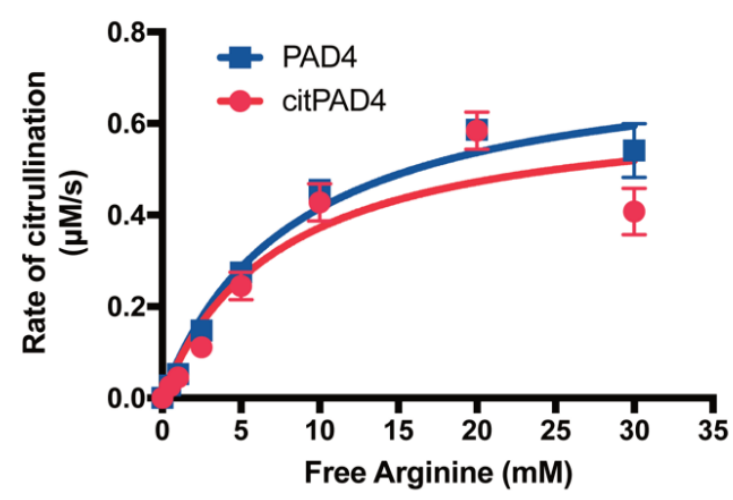

B

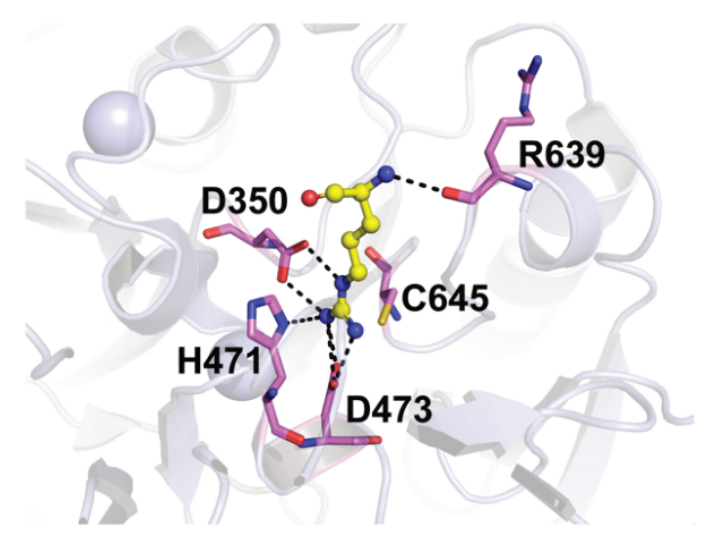

Figure 3. Comparison of PAD4 and citPAD4 activity towards free arginine substrate. $(A)$ Kinetics of the citrullination of free arginine by PAD4 and citPAD4. PAD4/citPAD4 (400nM) was incubated with a range of concentration (0$30 \mathrm{mM}$ ) for $10 \mathrm{~min}$. (B) Graphic representation of PAD4 interacting with free arginine predicted by docking using Sybylx-Surflex program. Substrates are represented in yellow balls and sticks, whereas key residues for interacting with the substrate are represented by magenta sticks. H-bond interactions are indicated with black dotted lines. 
activity of citPAD4 is thus not significantly different from that of non-citrullinated PAD4. These experimental findings were supported by in silico docking experiments showing that free arginine interacted with D350, H471, D473 and the backbone atoms of R639 (Figure 3B). Therefore, a conversion of R372, R374, and R639) to citrulline residue appears not to affect the PAD4 catalytic activity and the binding of arginine to PAD4.

\subsubsection{Peptidic substrate, $0.503 \mathrm{kDa}$}

We next investigated the activity of PAD4 and citPAD4 toward the peptide-based substrate CS-21(66), having a p-Glu-Pro-Arg-pNa sequence. Due to a comparatively limited availability of this substrate, as compared to the analysis of free arginine above, we measured the time-dependent citrullination production by PAD4 and citPAD4 at a fixed concentration of enzyme $400 \mathrm{nM}$ and maximally reachable substrate concentration of $2 \mathrm{mM}$. Again, PAD4 and citPAD4, under the reaction conditions chosen, generated similar amounts of citrulline during the incubation (Figure 4A), in agreement with the observations that were done for the analysis of free arginine. We employed molecular docking to study the interaction between CS-21(66) and the active site of PAD4 and citPAD4. Backbone atoms of residue Arg, pGlu and pNa, and the side chain of pNa residue of CS-21(66) were found to be able to interact with the side chains of R374, R639 of PAD4, respectively (Figure 4B). However, these interactions are not directly involved in the citrullination mechanism to convert arginine to citrulline residue. Hence, these results indicate that citrullination of arginine residues at the binding pocket (i.e. R374 and R639) does not affect PAD4 activity toward peptidic substrate.

CS-21(66) sequence: p-Glu-Pro-Arg-pNa<smiles>N=C(N)NCCC[C@H](NC(=O)[C@H]1CCCN1C(=O)C1CCC(=O)N1)C(=O)Nc1ccc([N+](=O)[O-])cc1</smiles>

A

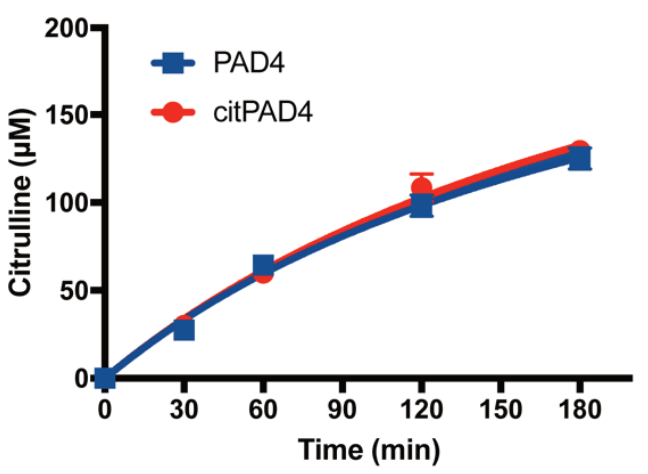

B

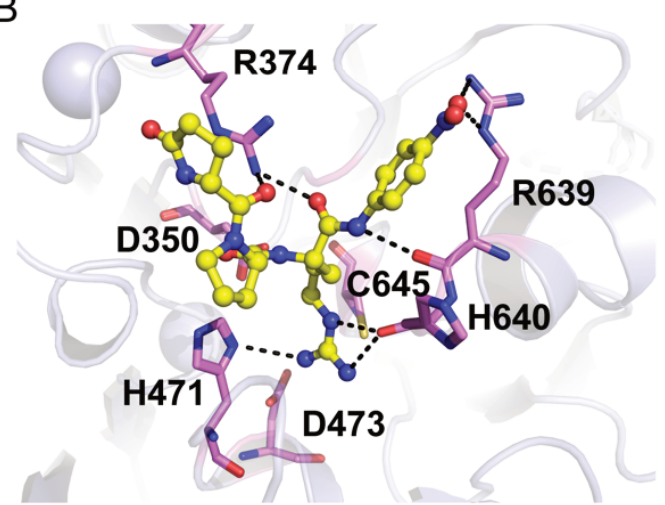

Figure 4. Comparison of PAD4 and citPAD4 activity towards medium (CS-21(66)) substrate. (A) Time course of citrulline production catalyzed by 400 nM PAD4 or citPAD4 using 2 mM arginine-containing peptide CS-21(66) as substrates. (B) Graphic representation of PAD4 interacting with CS-21(66) predicted by docking using SybylxSurflex program. Substrates are represented in yellow balls and sticks, whereas key residues for interacting with the substrate are represented by magenta sticks. H-bond interactions are indicated with black dotted lines. 


\subsubsection{Protein substrate (histone $H 3,15.4 \mathrm{kDa}$ )}

Citrullination of histone $\mathrm{H} 3$ was quantified by averaging of the densitometric results of five independent Western blot experiments. Our data (Figure 5A) show that citrullination by PAD4 or citPAD4 was not different at any of the tested histone $\mathrm{H} 3$ concentrations (Figure 5B). The results for histone $\mathrm{H} 3$ are in agreement with those obtained for the small arginine substrate and the peptidic substrate discussed above and confirm that autocitrullination of PAD4 does not alter its activity, including that towards its natural protein substrates such as histone $\mathrm{H} 3$.

A

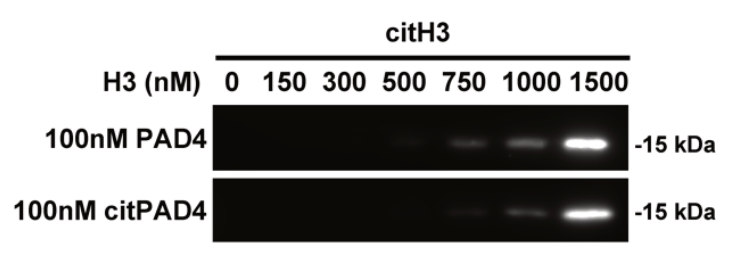

B

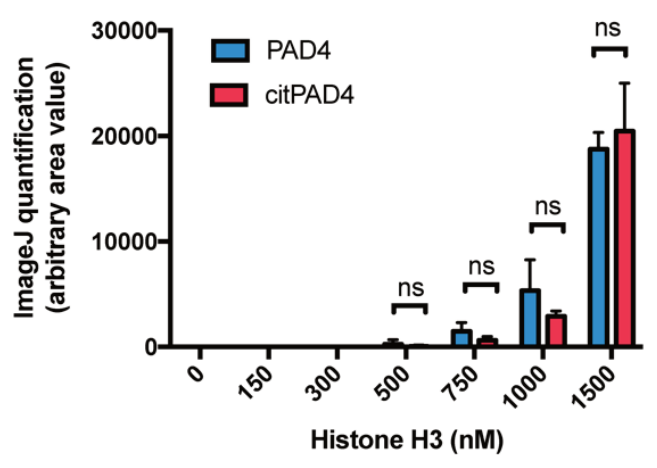

Figure 5. Comparison of PAD4 and citPAD4 activity towards histone H3. The comparison of PAD4 and citPAD4 activity was done by incubating $100 \mathrm{nM}$ of PAD4/citPAD4 with different amount of histone H3 (0-1500 nM) for 10 minutes. One representative western blot (of five times of independent experiments) is shown in $A$. The quantified citrulline signal produced by PAD4 and citPAD4 is shown in B which was calculated based on five independent Western blots.

\subsubsection{Evaluation of PAD4 and citPAD4 stability by structural bioinformatics}

\subsubsection{Dynamics of structural variation}

Replacement of a positively charged arginine by a neutral citrulline may alter intramolecular protein interactions resulting in a change of structural stability and conformation of PAD4. To examine PAD4 and citPAD4 stability we performed MD simulations of PAD4/citPAD4 and next evaluated the stability of PAD4/citPAD4 by monitoring the root mean square deviation (RMSD) of all heavy atoms during the simulations. The RMSD values for the complete PAD4 and citPAD4 structure during the simulation is shown in Figure 6 and exhibits small structural fluctuations of around 2.5-3.5 $\AA$. When considering the binding pocket only, RMSD values were very stable at $2.5 \AA$ for both PAD4 and citPAD4 (Figure 6). These results indicate that PAD4 and citPAD4, and especially the binding pocket, are stable during MD simulation. 
A

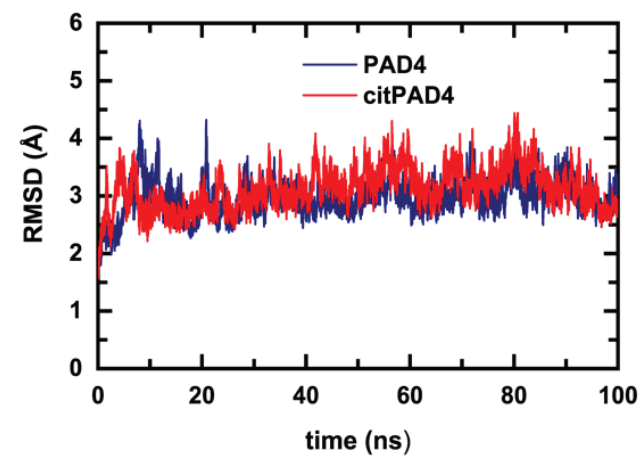

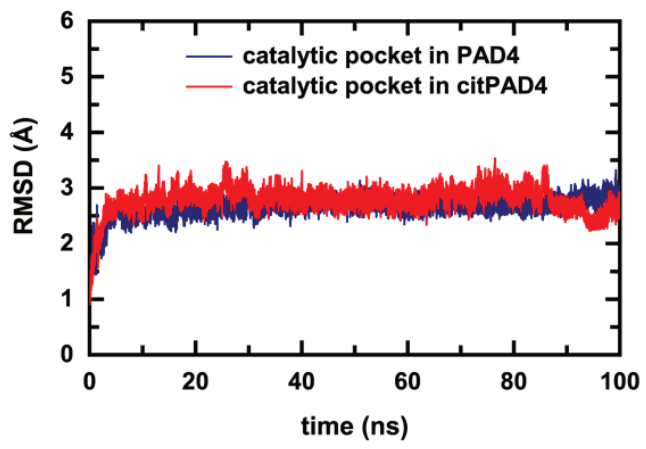

Figure 6. The RMSD of PAD4/citPAD4. (A) RMSD of the entire structure of PAD4 and citPAD4, (B) RMSD of the catalytic binding pocket of PAD4 and citPAD4. The RMSD was calculated based on the heavy atoms of residues.

\subsubsection{Principle components analysis (PCA)}

To further analyze potential conformational changes in PAD4 and citPAD4, we monitored the motions of PAD4 and citPAD4 during the MD simulation by a combination of principle components analysis, free energy landscape calculation and clustering analysis. The principal components analysis and free energy landscape point out that an ensemble of stable protein structures exists, as indicated by the darker colored free energy landscape contours in Figure 7A and 7B. PAD4 has one lowest energy basin I and citAD4 has two lowest energy basins II and III. These results imply that the PAD4 structures were more concentrated and stable, whilst the citPAD4 structures can adopt two alternative stable conformational states. However, by visualization and superimposition of the representative conformations of PAD4 and citPAD4 (basins I, II and III), we found that the aligned structures superpose mostly well except at the $\mathrm{N}$-terminal subdomain I and some surface loops while residues at the binding pocket exhibit low RMSD values (Figure 7C and 7D). 
A
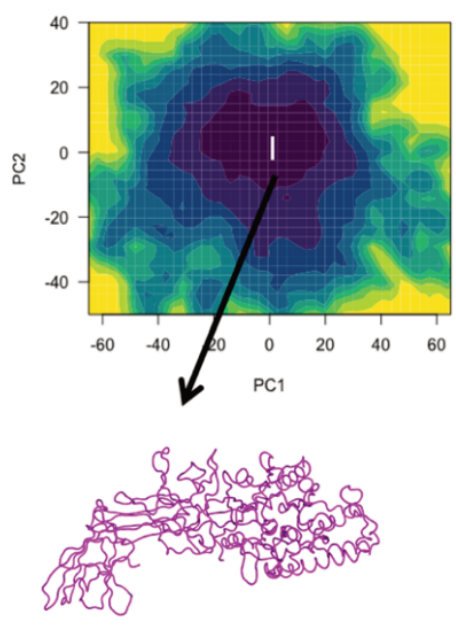

B

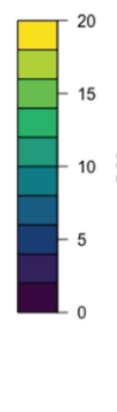

$20-$

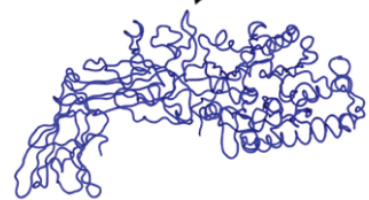

C1

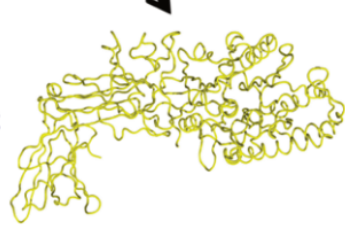

aa: 340

C

aa: 219-222

aa: $399-400$

aa: $636-638$

aa: 1-100

aa: $278-282$

$\mathrm{D}$
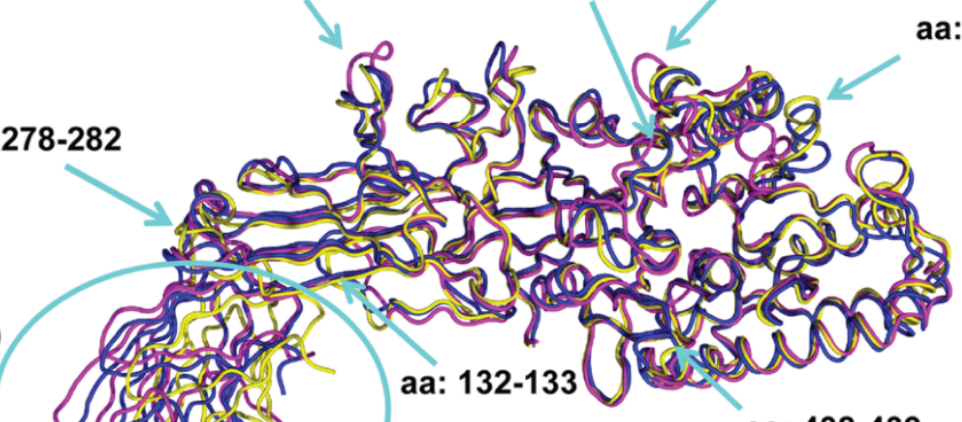

aa: $438-439$

D

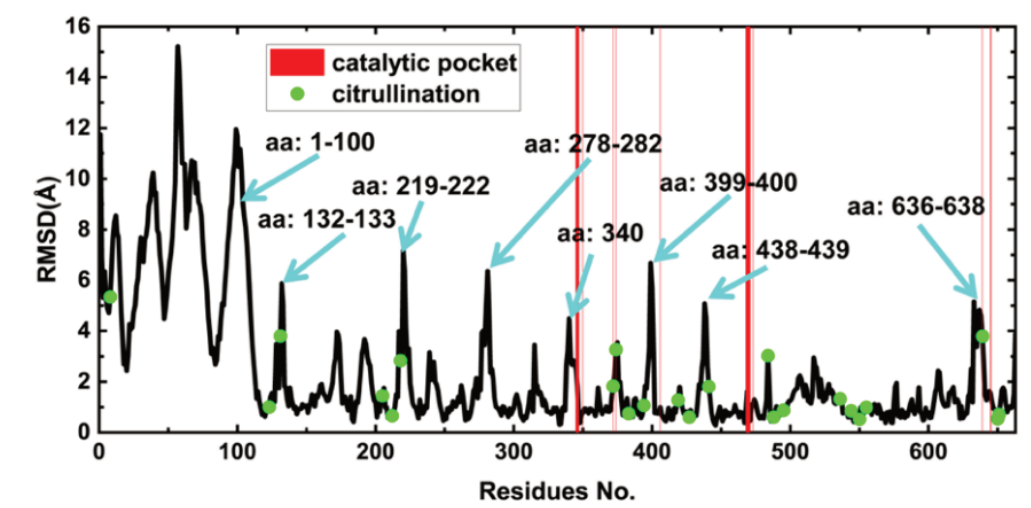

Figure 7. Relative free energy landscape (FEL) of PAD4 and citPAD4. FEL of PAD4 (A) and citPAD4 (B) determined by principal components analysis (PCA) using PC1 and PC2 projected with the trajectories extracted along the $M D$ simulations based on the $\mathrm{C} \alpha$ atom. The contour was filled with energy value $(\mathrm{kcal} / \mathrm{mol})$. The scale of energy is shown at the right of panel $(A)$ and $(B)$, with deep blue to yellow indicating low to high Gibbs free energy conformations, respectively. The contour map shows the motions of PAD4/citPAD4 with lowest free energy basin I, II, and III, corresponding to where the most representative structures were obtained. The obtained representative structures from I, II, and III by clustering based on Ca atoms are superposed together and shown in $c$ with magenta, blue, and yellow color, respectively (C). The calculated RMSD (Ca atoms) per residue between the representative structures of PAD4 (basin I) and citPAD4 (basin II and III) is shown in (D). Green dots represent the citrullination positions. Cyan arrows point regions with high RMSD values and deviation after aligning the $3 D$ structures. Red lines indicate residues at the binding pocket. 


\subsubsection{Investigation of a histone-derived peptide binding to PAD4 and citrullinated PAD4}

The experimental results indicate that autocitrullination of PAD4 does not alter its enzymatic activity toward the different substrates tested. To gain additional insight into the molecular interactions at the atomic level between a substrate and PAD4/citPAD4, we performed MD simulations of PAD4/citPAD4 in complex with a substrate. Since co-crystallized structures of a complex between PAD4 and a histone $\mathrm{H} 3$-derived peptide are available from the PDB, we selected the PAD4-R8H3 (sequence 6-TARKS-10) complex (PDB Code 2DEW) as a representative structure to investigate atomistic interactions between PAD4/citPAD4 and their substrate.

\subsubsection{Structural stability of PAD4- and citPAD4-substrate complexes}

The RMSD values of the PAD4/CitPAD4-R8H3 complexes Figure $8 \mathrm{~A}$ ) display a fluctuation in the range of $2 \AA$ to $4 \AA$ for both the PAD4 and citPAD4 complexes, implying that PAD4 is stable upon binding of the substrate and that autocitrullination does not influence the stability of the enzyme-substrate complex.

A

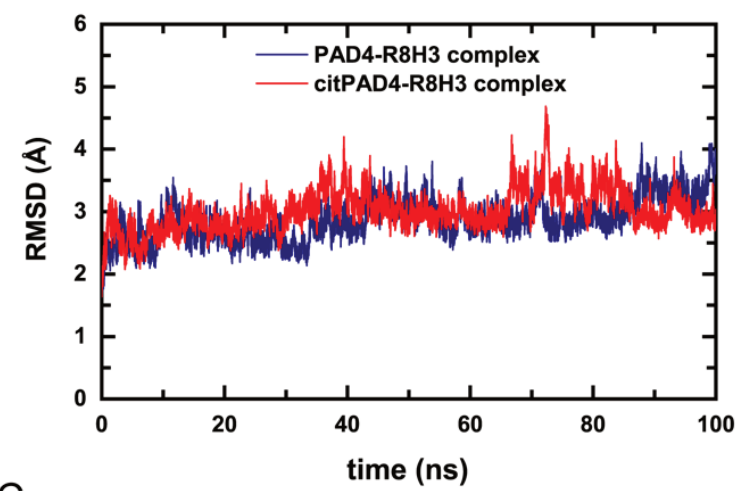

C

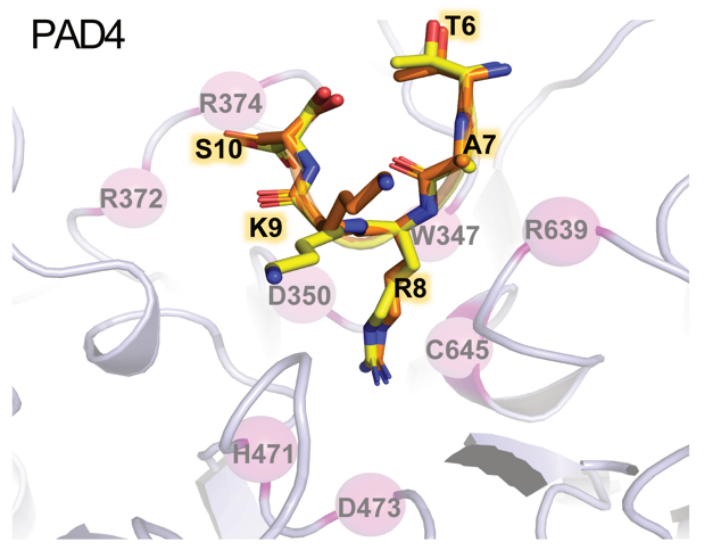

B

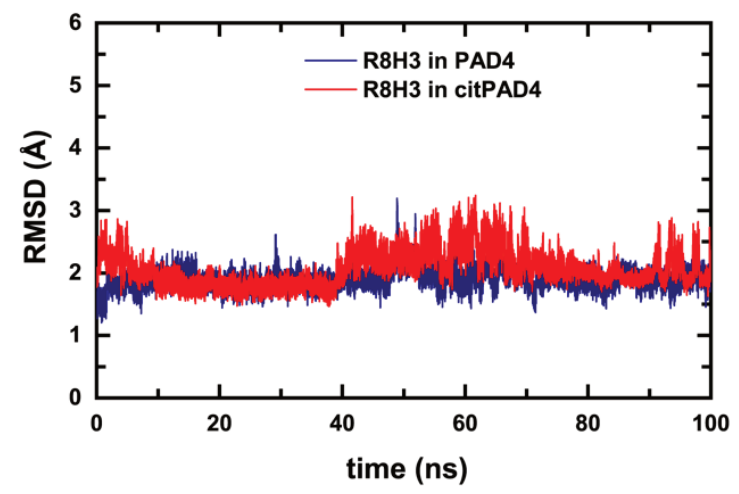

D

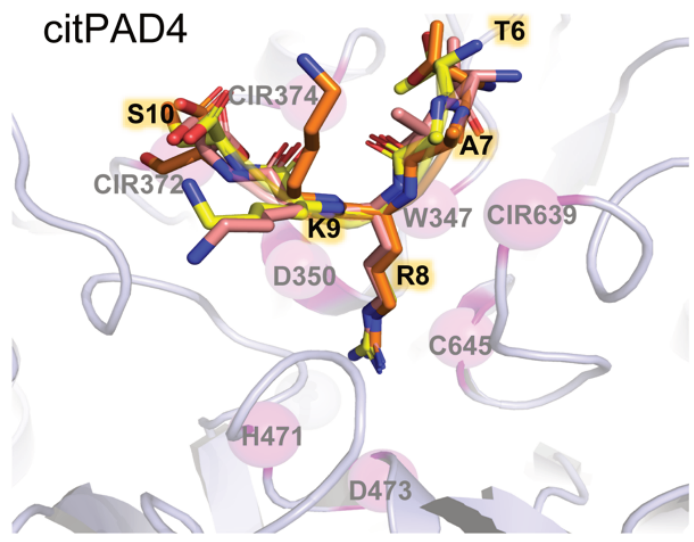

Figure 8. RMSD and conformation of R8H3 in complex with PAD4 or citPAD4. The RMSD of the PAD4-R8H3 and citPAD4-R8H3 complexes is shown in (A), the RMSD of the substrate R8H3 only is shown in (B). Representative $\mathrm{R} 8 \mathrm{H} 3$ conformations in PAD4 and citPAD4 were obtained by clustering based on all heavy atoms and superposed in $(C)$ and $(D)$, respectively. Superposed $R 8 H 3$ is represented as sticks; key residues for catalysis and binding are presented by magenta spheres in PAD4 and red in citPAD4.

The RMSD values for the substrate (Figure 8B) fluctuated slightly between $1 \AA$ and $3 \AA$ in both the PAD4 and citPAD4 complexes, which indicates a stable binding of R8H3 in the binding pocket of PAD4 or citPAD4. To investigate potential conformational changes of the substrate, we additionally compared 88 
representative structures of $\mathrm{R} 8 \mathrm{H} 3$, which were obtained after clustering analysis based on the heavy atoms of R8H3. Two large clusters were identified for PAD4-R8H3 complex, whereas three large clusters were obtained for the citPAD4-R8H3 complex. By superposing the representative structures of $\mathrm{R} 8 \mathrm{H} 3$ in each complex system (Figure $\mathbf{8 C}$ and $\mathrm{D}$ ), we found that the key residue R8, where the citrullination reaction takes place, is very well aligned inside the catalytic pocket of both PAD4 and citPAD4; neighboring residues such as T6, 7A, K9, and S10 exhibit a slight deviation.

By performing clustering analysis based on the complexes, we obtained a favorable binding mode of $\mathrm{R} 8 \mathrm{H} 3$ in the binding pocket of PAD4 (Figure 9A) and citPAD4 (Figure 9B). Structural analysis of these binding poses revealed that the guanidine group of R8 forms interactions with D350, D473 in both PAD4 and citPAD4 (Figure 9A and 9B). Hydrogen bond (H-bond) interactions between R8 and R639 (CIR639 in citPAD4) through backbone atoms were observed for both PAD4 and citPAD4. The neighboring residues of R8 (e.g. T6, K9 and S10) display different interactions with residues at the entrance of the binding pocket of PAD4/citPAD4. For example, the $\mathrm{S} 10$ substrate residue can form $\mathrm{H}$-bonds with R372 and R374 in PAD4 but these interactions were not observed in the citPAD4-substate complex. Nevertheless, R372 and R374 are not involved in the citrullination catalysis mechanism. Thus, citrullination of R372, R374 and R639 has limited effect on the activity of PAD4 since these residues do not directly form interactions with the side chain of R8.

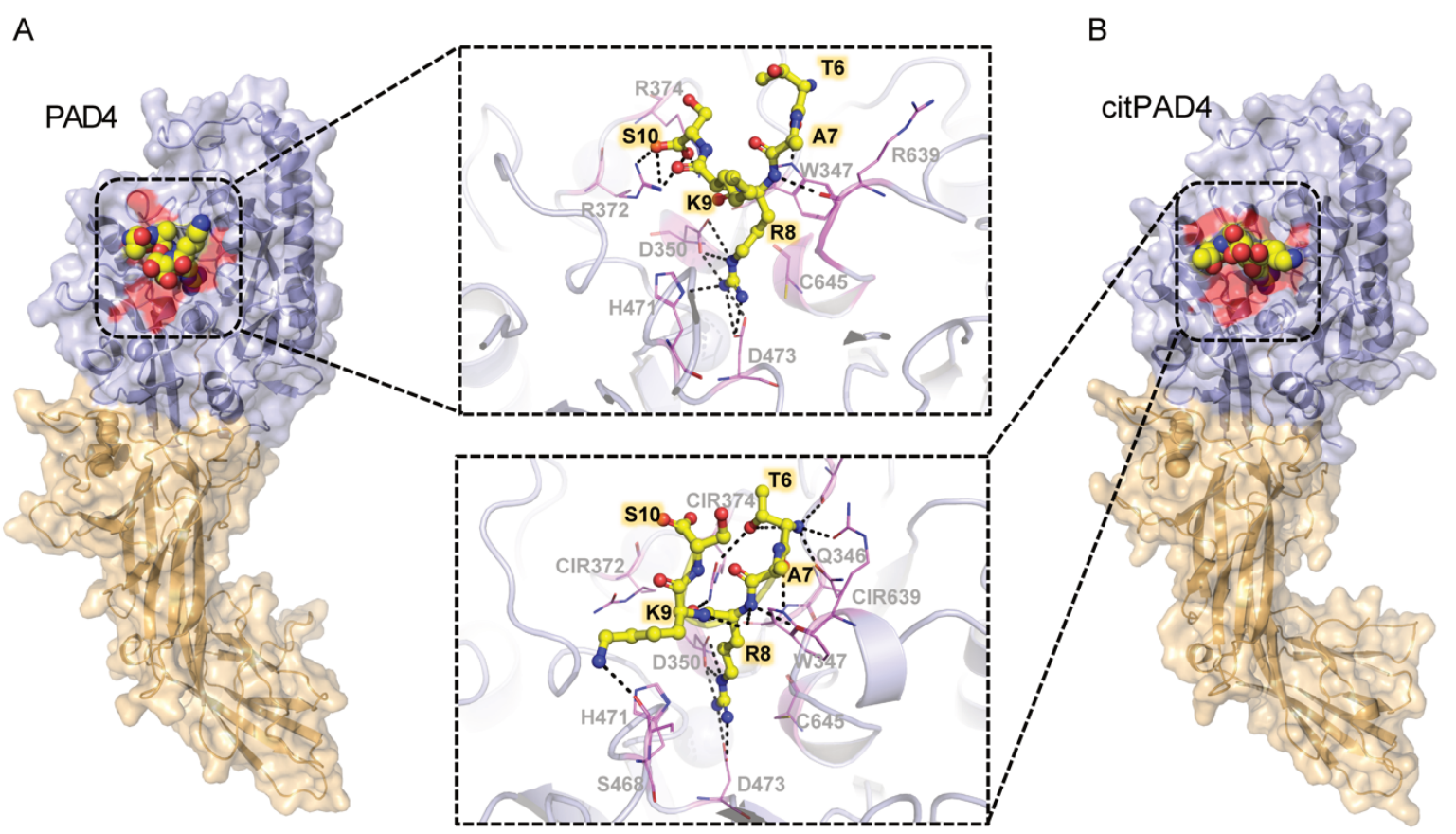

Figure 9. The representative binding poses of R8H3 in PAD4 (A) and citPAD4 (B). The representative structures of $P A D 4-R 8 H 3$ and citPAD4-R8H3 complex were obtained by clustering over 100ns MD simulations based on all heavy atoms. Lightorange and lightblue indicate N-terminal domain and C-terminal domain of PAD4, respectively. The binding pocket is indicated as a red area. Substrate R8H3 is represented as yellow colored sticks or balls; interactive residues are represented as magenta sticks. Black dashed lines indicate $\mathrm{H}$-bond between $\mathrm{R} 8 \mathrm{H} 3$ and $P A D 4 / c i t P A D 4$. 


\subsubsection{Characterization of the interaction between R8H3 substrate and PAD4 and citPAD4}

The total BFEs of the bound substrate R8H3 with PAD4 and citPAD4 were quite comparable, $-45.99 \pm 7.63$ and $-49.91 \pm 4.45 \mathrm{kcal} / \mathrm{mol}$ (Figure 10A), respectively, which indicates that the substrate $(\mathrm{R} 8 \mathrm{H} 3)$ has no preference for binding to either PAD4 or citPAD4. These results are in good agreement with our experimental data demonstrating that the citrullination of histone $\mathrm{H} 3$ as catalyzed by PAD4 or citPAD4 is not significantly different. Per-residue energy decomposition (DC) analysis revealed that the residues that have major contribution to binding with PAD4 or citPAD4 are W347, D350, D473 as their contribution levels were lower than $-4 \mathrm{kcal} / \mathrm{mol}$ (Figure 10B). Upon comparison between PAD4 and citPAD4, we found that residues R372 and R639 differ in the extent of their contribution to binding of R8H3. Whilst R372 of PAD4 contributed in PAD4 with $-5.92 \pm 1.69 \mathrm{kcal} / \mathrm{mol}$, this residue (CIR372) of citPAD4 exhibits only a small contribution $(-0.01 \pm 0.47 \mathrm{kcal} / \mathrm{mol})$ to the interaction with the substrate. On the other hand, R639 of PAD4 did not play an important role in substrate interaction $(-0.61 \pm 0.69 \mathrm{kcal} / \mathrm{mol})$ whereas this residue (CIR639) in citPAD4 contributed significantly to substrate binding $(-4.84 \pm 0.34 \mathrm{kcal} / \mathrm{mol})$. The binding free energies of citPAD4 gained by R639 compensates for the loss at R372 which results in a comparable overall binding free energy for the PAD4 and citPAD4 complexes. The contribution of R374/CIR374 in PAD4 and citPAD4 to binding with the substrate is relatively small $(-2.36 \pm 2.68$ and $-1.6 \pm 0.56 \mathrm{kcal} / \mathrm{mol}$, respectively). In conclusion, these results indicate that citrullination of arginine may influence the contributions of the binding free energy for individual amino acid residues, in particular for arginine or citrulline residues, but it does not change the overall binding of the PAD4/citPAD4 with the substrate.

A

\begin{tabular}{cc}
\hline system & $\begin{array}{c}\text { Binding free energy } \\
(\mathrm{kcal} / \mathrm{mol})\end{array}$ \\
\hline PAD4-R8H3 & $-45.99 \pm 7.63$ \\
citPAD4-R8H3 & $-49.91 \pm 4.45$ \\
\hline
\end{tabular}

B

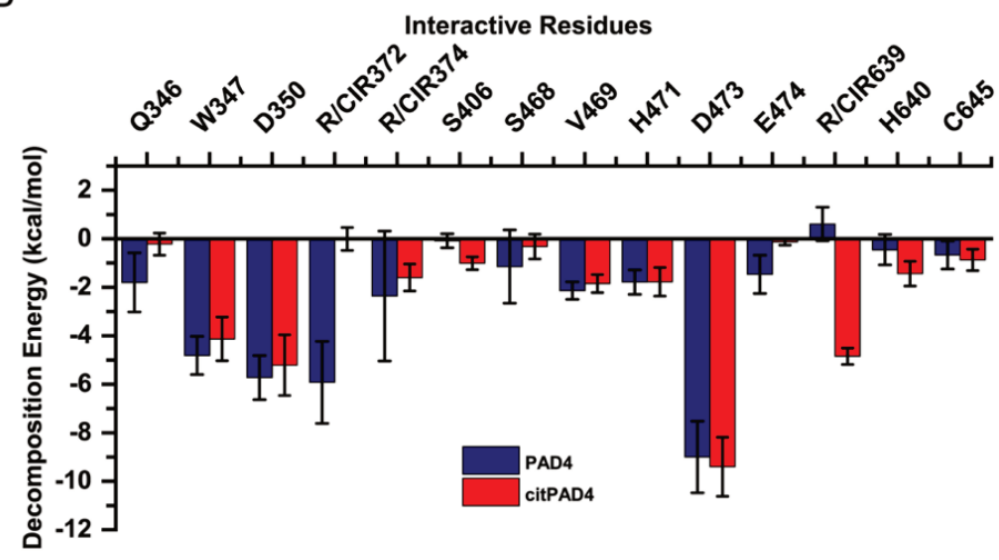

Figure 10. Binding free energy and decomposition (DC) analysis of R8H3 with PAD4 or citPAD4. (A) The total binding free energy (MM/GBSA) of PAD4-R8H3 and citPAD4-R8H3 complex, (B) Decomposition (DC) analysis of $P A D 4 / c i t P A D 4-R 8 H 3$ complex. CIR represents the non-standard citrulline residue. 


\subsubsection{Hydrogen bond analysis}

We additionally monitored $\mathrm{H}$-bond occupancy between the substrate $(\mathrm{R} 8 \mathrm{H} 3)$ and PAD4/citPAD4 during the simulations. The total number of key $\mathrm{H}$-bond pairs between the substrate and PAD4 or citPAD4 observed in both complexes are similar (11 pairs for PAD4 and 12 pairs for citPAD4 as summarized in

Table 2). Similar results were observed for PAD4-R8H3 and citPAD4-R8H3 complexes; for example, major interactions arose from $\mathrm{H}$-bond interactions between residue $\mathrm{R} 8$ (of $\mathrm{R} 8 \mathrm{H} 3$ ) with $\mathrm{D} 473$ (42.1-56.0\% in PAD4 and 39.8-58.3\% in citPAD4) and also with D350 (69.0-92.8\% in PAD4 and 18.0-77.3\% in citPAD4). The neighboring residues around R8 (i.e. backbone oxygen of T6) can also form a H-bond with the nitrogen in the sidechain of W347 in both PAD4 (62.7\%) and citPAD4 (35.9\%) complexes (Table 2). Additional $\mathrm{H}$-bond interactions between the backbone atom of $\mathrm{S} 10(\mathrm{R} 8 \mathrm{H} 3)$ with the side chain of R372 (25.3-34.6\%) and R374 (16.2\%) of PAD4 were observed, but these H-bond pairs (S10CIR372/374) were not detected in citPAD4. MD simulations and $\mathrm{H}$-bond occupation analysis of the histone H3-derived peptide (R8H3) with PAD4/citPAD4 support our experimental findings with the peptidic substrate and those from molecular docking (section Peptidic substrate, $0.503 \mathrm{kDa}$ and Figure 4), confirming that the R/CIR639 interaction with R8 (R8H3) is brought about mainly through backbone-to-backbone interaction and that R372 and R374 do not directly interact with R8 (R8H3) but with neighboring residues aside R8. Thus, modification of the side chain of R372, R374, and R639 to citrulline residue has limited effect on the binding of PAD4 to its substrate, and therefore will have little to no influence on the activity of PAD4.

Table 2. Hydrogen bonds between PAD4/citPAD4 and histone R8H3 peptide (6-TARKS-10). Hydrogen bonds were calculated over 100ns of MD simulations. Hydrogen bond occupations $>15 \%$ are reported.

\begin{tabular}{|c|c|c|c|c|c|c|c|}
\hline \multicolumn{4}{|c|}{ PAD4-R8H3 } & \multicolumn{4}{|c|}{ citPAD4-R8H3 } \\
\hline Acceptor & DonorH & Donor & $\%$ occupation & Acceptor & DonorH & Donor & $\%$ occupation \\
\hline T6@O & W347@HE1 & W347@NE1 & 62.7 & T6@O & W347@HE1 & W347@NE1 & 35.9 \\
\hline s10@o & R372@HH12 & R372@NH1 & 34.6 & - & & & \\
\hline S10@o & R372@HH22 & R372@NH2 & 25.3 & - & & & \\
\hline S10@o & R374@HH21 & R374@NH2 & 16.2 & - & & & \\
\hline- & & & & R8@O & CIR374@HH12 & CIR@374NH1 & 41.2 \\
\hline- & & & & D345@OD1 & T6@HG1 & T6@OG1 & 90.1 \\
\hline D350@OD1 & R8@HH21 & R8@NH2 & 92.8 & D350@OD1 & R8@HH21 & R8@NH2 & 77.3 \\
\hline \multirow[t]{3}{*}{ D350@OD1 } & R8@HE & R8@NE & 69.0 & D350@OD1 & R8@HE & R8@NE & 39.7 \\
\hline & & & & D350@OD2 & R8@HE & R8@NE & 19.0 \\
\hline & & & & D350@OD2 & R8@HH21 & R8@NH2 & 18.0 \\
\hline D473@OD1 & R8@HH12 & R8@NH1 & 56.0 & D473@OD1 & R8@HH12 & R8@NH1 & 58.3 \\
\hline D473@OD1 & R8@HH22 & R8@NH2 & 40.4 & D473@OD1 & R8@HH22 & R8@NH2 & 40.1 \\
\hline D473@OD2 & R8@HH22 & R8@NH2 & 49.8 & D473@OD2 & R8@HH22 & R8@NH2 & 57.2 \\
\hline D473@OD2 & R8@HH12 & R8@NH1 & 42.1 & D473@OD2 & R8@HH12 & R8@NH1 & 39.8 \\
\hline R639@o & R8@H & R8@N & 32.4 & CIR639@O & R8@H & R8@N & 42.5 \\
\hline
\end{tabular}




\subsection{Discussion}

Previous studies on autocitrullination of PAD410,11 have reported contrasting results and the exact effects of autocitrullination on PAD4 activity and its role in physiological conditions remains unresolved. To determine whether autocitrullination influences the enzymatic activity of PAD4, we have employed both in vitro and in silico approaches to investigate the activity of PAD4 and citPAD4 and their interaction with substrates. We demonstrated that autocitrullination does not alter PAD4's enzymatic activity towards the substrates tested.

PAD4 citrullinates itself in a time- and concentration-dependent manner. Notably, no significant difference was identified when comparing autocitrullinated PAD4 at 1 hour and 2 hour incubation time by Western blot technique, which suggests that the autocitrullination of PAD4 already approaches saturation after $1 \mathrm{~h}$ under the conditions chosen here. Via MS analysis, we observed that 14/27 arginine residues were citrullinated within 1 hour of incubation whereas 23/27 of arginine residues (85\%) were citrullinated within 2 hours of incubation. This percentage may increase even further after prolonged incubation time. An explanation for this observed discrepancy between the results of these two different experimental approaches likely resides in the different characteristics of Western blotting and MS. The sensitivity and linearity in signal are clearly different between MS and western blot-based measurements, and because the western blotting method cannot always distinguish proteins with different degrees of citrullination, given that antibodies recognizing citrulline, due to their size, will compete for binding to adjacent citrullinated arginines. It is worth to mention that the mass spectrometry analysis performed might not be sensitive enough to detect all citrullines. Despite our use of two different proteases for PAD4 fragmentation, one could potentially improve detection by including additional proteases, which might increase the likelihood of citrulline detection via mass spectrometry. The neighboring residues around potential citrulline residues are listed for every arginine site since it was reported earlier that the position of an arginine in a protein sequence and the nature of neighboring residues around the target arginine influences the catalysis by PAD429. However, no preferential properties of neighboring residues aside targeted arginine have been observed by us here.

We investigated the kinetic properties of PAD4 and citPAD4 towards substrates of different size. Free arginine was used as small-sized substrate since we found that PAD4 is able to convert free L-arginine to L-citrulline, which is contrast to previous reports ${ }^{29}, 30$. Further, a short arginine-containing peptide, CS21(66), was chosen as medium-sized PAD4 substrate. For a larger, protein substrate, nuclear histone $\mathrm{H} 3$ was selected as it is known that PAD4 can citrullinate histone $\mathrm{H} 3$ at multiple sites, such as at R2, $\mathrm{R} 8, \mathrm{R} 17$ and $\mathrm{R} 26$ located at the histone $\mathrm{H} 3 \mathrm{~N}$-terminus ${ }^{31,32}$. Our in vitro results show that the activity of citPAD4 is similar as PAD4, which is in agreement with a previous study by Slack and coworkers ${ }^{11}$.

Consistent with our experimental findings, the in silico calculated binding free energies of substrate binding to PAD4 and citPAD4 are similar. From the decomposition analysis, the key residues that contribute to the binding of PAD4 to its substrate are residues W347, D473 and D350. Amongst, these D350 and D473 are crucial for the regulation of PAD4 activity as shown by mutagenesis study ${ }^{25}$. For 92 
residues (R372, $\mathrm{R} 374$, and $\mathrm{R} 639$ ) located at the entrance of the substrate binding pocket we hypothesized that citrullination of these residues may influence the binding of a substrate to PAD4/citPAD4. However, our in silico studies point out that these residues do not exhibit direct interactions with the side chain of $\mathrm{R} 8$ of the substrate where the citrullination reaction takes place. The residues $\mathrm{R} 372, \mathrm{R} 374$, and $\mathrm{R} 639$ bind and interact with the backbone of $\mathrm{R} 8$ or neighboring residues assisting to stabilize the binding of the substrates into binding pocket of PAD4/citPAD4. Taken together, modification of the side chains of R372, R374 and R639 by autocitrullination should have only minor or no effect on the PAD4-substrate interaction. Given that we did not determine the effect of autocitrullination of PAD4 in its physiological environment, we cannot exclude that considering the large change in PAD4 surface charges, it is still possible that autocitrullination changes the binding of PAD4 to other protein partners. Thereby, autocitrullination could indirectly influence PAD4 activity by allowing the blocking of the active site. However, such possibility needs to be confirmed by in vivo study.

\subsection{Conclusion and future perspective}

We conclude that autocitrullination does not affect PAD4 activity when tested on three different sized substrates in vitro, and this has been confirmed by in silico studies which revealed that autocitrullination does not influence the structural stability of the substrate binding pocket of PAD4. Therefore, we conclude that autocitrullination does not represent a self-control mechanism that regulates PAD4 enzymatic activity per se, and that more studies are needed to investigate the role of autocitrullination in vivo. We have demonstrated that the application and combination of in vitro and in silico methods can be used to study and decipher interactions at the molecular level as well as to gain insight into interaction atomic levels. These approaches can be widely utilized to study and investigate protein-protein interactions/complexes.

\subsection{Abbreviations}

BAEE N-a-benzoyl L-arginine ethyl ester

BFE Binding Free Energy

COLDER COlor DEvelopment Reagent

citPAD4 citrullinated PAD4

EDTA Ethylenediaminetetraacetic acid

GB Generalized Born

HDAC1 Histone deacetylase 1

HRP Horseradish peroxidase

IgG Immunoglobulin $\mathrm{G}$

MM/GBSA Molecular Mechanics/ Generalized Born and Surface Area continuum

PAD4 Protein arginine deiminase 4

PDB Protein Data Bank 


$\begin{array}{ll}\text { PPIs } & \text { protein-protein interactions } \\ \text { PRMT1 } & \text { protein arginine methyltransferase } 1 \\ \text { MD } & \text { Molecular Dynamics } \\ \text { R8H3 } & \text { peptide 6-TARKS-10 from the N-terminus of histone H3 } \\ \text { RMSD } & \text { Root Mean Square Deviation } \\ \text { SDS } & \text { Sodium Dodecyl Sulphate } \\ \text { TBS } & \text { Tris Buffered Saline } \\ \text { TCEP } & \text { Tris(2-carboxyethyl)phosphine }\end{array}$

\subsection{Acknowledgements}

We thank the China Scholarship Council for financial support to Ms Xiaosong.Liu. We thank Freek G. Bouwman for Mass spectrometry analysis. 


\section{References}

1. Nakashima, K., et al., Nuclear localization of peptidylarginine deiminase $\mathrm{V}$ and histone deimination in granulocytes. J. Biol. Chem. 2002, 277 (51), 49562-49568.

2. Papayannopoulos, V., Neutrophil extracellular traps in immunity and disease. Nature Reviews Immunology 2018, 18 (2), 134-147.

3. Suzuki, A., et al., Decreased severity of experimental autoimmune arthritis in peptidylarginine deiminase type 4 knockout mice. BMC Musculoskelet. Disord. 2016, 17, 205-205.

4. Chang, X.; Han, J., Expression of Peptidylarginine Deiminase Type 4 (PAD4) in Various Tumors. Mol. Carcinog. 2006, 45 (9), 183-196.

5. Fuchs, T. A., et al., Neutrophil extracellular trap (NET) impact on deep vein thrombosis. Arterioscler. Thromb. Vasc. Biol. 2012, 32 (8), 1777-1783.

6. Neeli, I., et al., Histone Deimination As a Response to Inflammatory Stimuli in Neutrophils. The Journal of Immunology 2008, 180 (3), 1895-1902.

7. Mondal, S.; Thompson, P. R., Protein Arginine Deiminases (PADs): Biochemistry and Chemical Biology of Protein Citrullination. Acc. Chem. Res. 2019, 52 (3), 818-832.

8. Wang, Y., et al., Histone hypercitrullination mediates chromatin decondensation and neutrophil extracellular trap formation. J. Cell Biol. 2009, 184 (2), 205-213.

9. Tanikawa, C., et al., Citrullination of RGG Motifs in FET Proteins by PAD4 Regulates Protein Aggregation and ALS Susceptibility. Cell Rep. 2018, 22 (6), 1473-1483.

10. Andrade, F., et al., Autocitrullination of human peptidyl arginine deiminase type 4 regulates protein citrullination during cell activation. Arthritis Rheum. 2010, 62 (6), 1630-1640.

11. Slack, J. L., et al., Development and use of clickable activity based protein profiling agents for protein arginine deiminase 4. ACS Chem. Biol. 2011, 6 (5), 466-476.

12. Senshu, T., et al., Detection of citrulline residues in deiminated proteins on polyvinylidene difluoride membrane. Anal. Biochem. 1992, 203 (1), 94-100.

13. Bouwman, F., et al., A combination of protein profiling and isotopomer analysis using matrix-assisted laser desorption/ionization-time of flight mass spectrometry reveals an active metabolism of the extracellular matrix of 3T3-L1 adipocytes. PROTEOMICS 2004, 4 (12), 3855-3863.

14. Roumans, N. J. T., et al., Weight loss-induced cellular stress in subcutaneous adipose tissue and the risk for weight regain in overweight and obese adults. Int. J. Obes. 2017, 41 (6), 894-901.

15. Knipp, M.; Vasak, M., A colorimetric 96-well microtiter plate assay for the determination of enzymatically formed citrulline. Analytical biochemistry 2000, 286 (2), 257-264.

16. Pastra-Landis, S. C., et al., An improved colorimetric assay for aspartate and ornithine transcarbamylases. Analytical Biochemistry 1981, 118 (2), 358-363.

17. Sugawara, K., et al., Colorimetric determination of citrulline residues in proteins. Analytical biochemistry 1998, 265 (1), 92-96.

18. Krieger, E.; Vriend, G., YASARA View - molecular graphics for all devices - from smartphones to workstations. Bioinformatics (Oxford, England) 2014, 30 (20), 2981-2982.

19. Vanquelef, E., et al., R.E.D. Server: a web service for deriving RESP and ESP charges and building force field libraries for new molecules and molecular fragments. Nucl. Acids Res. (Web server issue) 2011, 39 , W511-W517.

20. Wichapong, K., et al., Structure-Based Design of Peptidic Inhibitors of the Interaction between CC Chemokine Ligand 5 (CCL5) and Human Neutrophil Peptides 1 (HNP1). Journal of Medicinal Chemistry 2016, 59 (9), 4289-4301.

21. Wichapong, K., et al., Rational modulator design by exploitation of protein-protein complex structures. Future Med. Chem. 2019, 11 (9), 1015-1033.

22. Case, D., et al., Amber 16. University of California, San Francisco, 2016.

23. Genheden, S.; Ryde, U., The MM/PBSA and MM/GBSA methods to estimate ligand-binding affinities. Expert Opin Drug Discov 2015, 10 (5), 449-461.

24. Miller, B. R., 3rd, et al., MMPBSA.py: An Efficient Program for End-State Free Energy Calculations. J. Chem. Theory Comput. 2012, 8 (9), 3314-21.

25. Arita, K., et al., Structural basis for $\mathrm{Ca}(2+)$-induced activation of human PAD4. Nat. Struct. Mol. Biol. 2004, 11 (8), 777-83.

26. Arita, K., et al., Structural basis for histone N-terminal recognition by human peptidylarginine deiminase 4 . Proceedings of the National Academy of Sciences 2006, 103 (14), 5291 LP-5296.

27. Luo, Y., et al., Inhibitors and Inactivators of Protein Arginine Deiminase 4: Functional and Structural Characterization. Biochemistry 2006, 45 (39), 11727-11736.

28. Jones, J. E., et al., Synthesis and screening of a haloacetamidine containing library to identify PAD4 selective inhibitors. ACS Chem. Biol. 2012, 7 (1), 160-165. 
29. Nomura, K., Specificity and mode of action of the muscle-type protein-arginine deiminase. Archives of Biochemistry and Biophysics 1992, 293 (2), 362-369.

30. Takahara, H., et al., Calcium-dependent Properties of Peptidylarginine Deiminase from Rabbit Skeletal Muscle. Agric. Biol. Chem. 1986, 50 (11), 2899-2904.

31. Cuthbert, G. L., et al., Histone deimination antagonizes arginine methylation. Cell 2004, 118 (5), 545-553.

32. Wang, Y., et al., Human PAD4 Regulates Histone Arginine Methylation Levels via Demethylimination. Science 2004, 306 (5694), 279-283. 


\section{Supplementary Information}

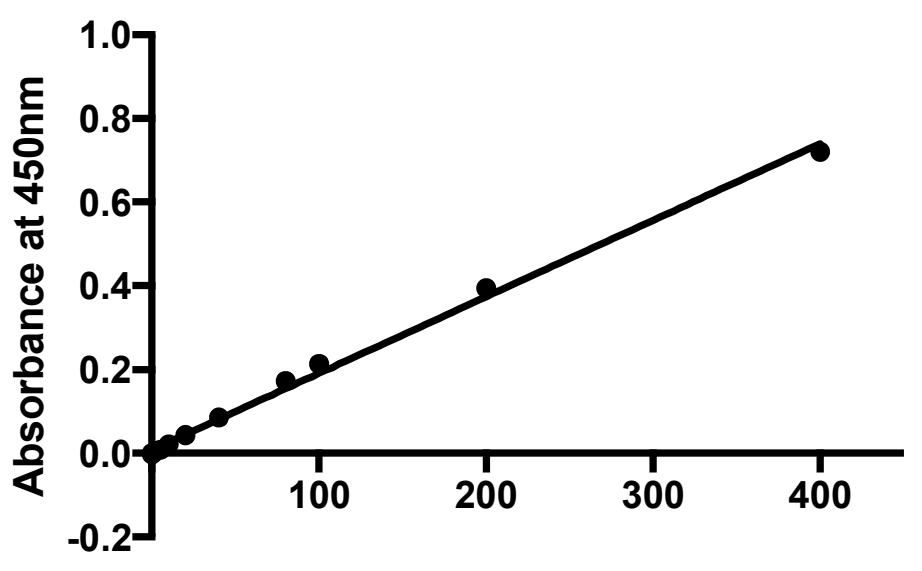

Citrulline ( $\mu \mathrm{M})$

Supplementary Figure 1. The standard curve of citrulline by COLDER assay. Various amount of L-citrulline (0-400 $\mu \mathrm{M}$ ) were dissolved in PAD4 reaction buffer (Tris $100 \mathrm{mM}, \mathrm{NaCl} 150 \mathrm{mM}, \mathrm{CaCl}_{2}$ 10mM, TCEP $1 \mathrm{mM}$ ). All measurements were performed in triplicates.

A citH3

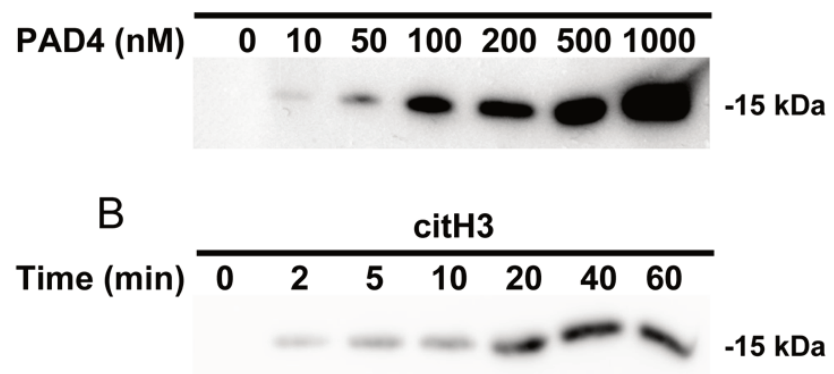

Supplementary Figure 2. Optimization of PAD4 concentration and reaction time for citrullinating histone $H 3$. The Histone H3 (1000 nM) citrullination by PAD4 was investigated by optimizing amount of PAD4 (0-1000 nM) (A) and citrullination time (0-60 $\mathrm{min})(B)$ to get initial reaction velocity. 



\section{Chapter}

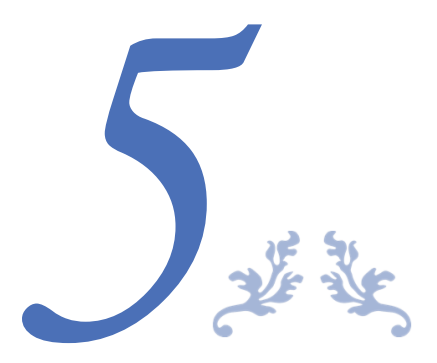

\section{Pseudonaja textilis venom-derived factor Va retains structural integrity following proteolysis by activated protein C}

Mark Schreuder ${ }^{1, *}$, Xiaosong Liu'2,, Ka Lei Cheung ${ }^{1}$, Pieter H. Reitsma ${ }^{1,3}$, Gerry A.F. Nicolaes ${ }^{2}$, and Mettine H.A. Bos ${ }^{1}$

${ }^{1}$ Division of Thrombosis and Hemostasis, Einthoven Laboratory for Vascular and Regenerative Medicine, Leiden University Medical Center, Leiden, The Netherlands.

2Department of Biochemistry, Cardiovascular Research Institute Maastricht (CARIM), Maastricht University, Maastricht, The Netherlands

${ }^{3}$ VarmX B.V, Leiden, The Netherlands.

${ }^{*}$ These authors contributed equally 


\begin{abstract}
The Australian snake Pseudonaja textilis comprises a venom-derived factor $\mathrm{V}$ variant (ptFV) that retains cofactor function despite activated protein $C$ (APC)-dependent proteolysis. Here we aimed to unravel the mechanistic principles by determining the role of the absent Arg306 cleavage site that is required for the inactivation of mammalian factor $\mathrm{Va}(\mathrm{FVa})$, in combination with a previously described unique disulfide bond that covalently links the FVa heavy and light chain. Our findings show that in contrast to human FVa, APC-catalyzed proteolysis of ptFVa at Arg306 and Lys507, and deletion of the unique disulfide bond does not abrogate ptFVa cofactor function. Remarkably, upon proteolysis the structural integrity of ptFVa is maintained indicating that stable non-covalent interactions prevent A2-domain dissociation and allow productive interactions with venom-derived factor $\mathrm{Xa}$. Using in silico approaches we uncovered key regions located in the $\mathrm{A} 1$ and $\mathrm{A} 2$ domain that are likely at the basis of this remarkable characteristic. Taken together, we report a completely novel role for specific A1 and A2 domain structural regions that have been uniquely adapted in $\mathrm{ptFVa}$ in order to prevent $\mathrm{A} 2$ domain dissociation. As such, these results challenge our current understanding by which strict regulatory mechanisms control $\mathrm{FVa}$ activity and could prove valuable in the generation of novel cofactor-based therapeutics for the treatment of bleeding disorders.
\end{abstract}

Keywords: Factor V, Snake Venoms, Activated Protein C Resistance, Blood Coagulation 


\subsection{Introduction}

Blood coagulation is a tightly regulated process composed of the sequential and concerted action of several serine proteases and their respective cofactors, together responsible for minimizing blood loss following vascular injury. Central to this process is the activation of coagulation factor $\vee(F V)$ into the active cofactor $\mathrm{Va}(\mathrm{FVa})^{1-3}$. Once $\mathrm{FV}$ is partially or fully activated, it functions as cofactor for the serine protease factor $\mathrm{Xa}(\mathrm{FXa})$ by formation of the prothrombinase complex on the surface of an anionic membrane in the presence of calcium ions ${ }^{4,5}$. This complex rapidly converts prothrombin to thrombin, a key regulatory enzyme in coagulation. The coagulation system can also be exploited to gain selective advantages. The procoagulant venom of several Australian Elapid snakes contains a powerful enzyme complex that specifically activates prothrombin in order to disrupt the prey's intrinsic hemostatic balance ${ }^{6-9}$. This prothrombin-activating complex consists of FX- and FV-like proteins ${ }^{10-13}$ comprising certain remarkable gain-of-function adaptations that enable this prothrombinase-like complex to initiate coagulation in an uncontrolled manner ${ }^{14-17}$. Previously we have demonstrated that venom FV derived from the common brown snake Pseudonaja textilis (ptFV), while undergoing selective proteolysis, is functionally resistant to FVa's natural inhibitor activated protein C (APC) $)^{11,14,18 .}$

To downregulate the procoagulant response, coagulant FVa is inactivated by APC via proteolysis of the A2 domain residues Arg306, Arg506, and Arg679 ${ }^{19-23}$. Cleavage at Arg306 and Arg506 results in A2 domain dissociation and subsequent loss of $\mathrm{FXa}$ affinity ${ }^{24}$. While ptFVa is cleaved at a position that is homologous to human Arg506 (Lys507) ${ }^{14}$, an Arg306-like cleavage site is absent ${ }^{11,}{ }^{14}$. Furthermore, venom $F V$ comprises a unique disulfide bond that covalently links the $A 2$ and $A 3$ domains ${ }^{14,16}$. Although we have previously reported that this disulfide bond is not responsible for the functional APC resistance ${ }^{18}$, whether it might stabilize the A2 domain binding affinity upon introduction of the Arg306 cleavage site remains to be determined.

In the present study, we generated several chimeric ptFV variants with the goal of uncovering the mechanistic principles of its exceptional functional resistance to APC. Our results demonstrate that ptFV has uniquely adapted several structural elements in order to prevent $\mathrm{A} 2$ domain dissociation and ptFVa inactivation. As such, these findings highlight the remarkable evolutionary adaptations that have made ptFVa into an extremely stable procoagulant venom and provide new insights in the structural requirements of $\mathrm{APC}$-induced $\mathrm{FVa}$ inactivation.

\subsection{Materials and Methods}

\subsubsection{Materials and Reagents}

The inhibitor dansylarginine- $\mathrm{N}$-(3-ethyl-1,5-pentanediyl)amide (DAPA) was from Haematologic Technologies (Essex Junction, VT). The peptidyl substrate H-D-Phe-Pip-Arg-pNA (S2238) was obtained from Instrumentation Laboratories (Bedford, MA, USA). All tissue culture reagents were from Life Technologies (Carlsbad, CA, USA). Small unilamellar phospholipid vesicles (PCPS) composed of $75 \%$ (wt/wt) hen egg L-phosphatidylcholine (PC) and $25 \%$ (wt/wt) porcine brain L-phosphatidylserine (PS) 
(Avanti Polar Lipids, Alabaster, AL, USA) were prepared and characterized as described previously ${ }^{25}$. FV-depleted human plasma and Neoplastine CI Plus 10 prothrombin time (PT) reagent were obtained from Diagnostica Stago (Paris, France). All functional assays were performed in HEPES buffered saline (HBS: $20 \mathrm{mM}$ HEPES, $0.15 \mathrm{M} \mathrm{NaCl}, \mathrm{pH}$ 7.5) supplemented with $5 \mathrm{mM} \mathrm{CaCl}_{2}, 0.1 \%$ PEG8000 and filtered over a $0.2 \mu \mathrm{m}$ filter (assay buffer).

\subsubsection{Proteins}

The human plasma-derived coagulation factors $\mathrm{FXa}$, prothrombin, $\alpha$-thrombin, APC and protein S were from Haematologic Technologies (Essex Junction, VT). Restriction endonucleases Xcml and Bsu36I were obtained from New England Biolabs (Ipswich, MA, USA). Recombinant venom-derived Pseudonaja textilis $\mathrm{FXa}$ (ptFXa) was prepared, purified, and characterized as described ${ }^{18}$. Recombinant constitutively active B-domainless human factor $\mathrm{V}$ (FV-810; hFV) and ptFV were prepared, purified, and characterized as described previously ${ }^{14}, 26$. The molecular weight and the extinction coefficients $\left(E_{0.1 \%, 280 \mathrm{~nm}}\right)$ of the newly generated hFV-pt306, ptFV-h306, and ptFV-h306 S-S were assumed to be equal to $\mathrm{hFV}(216,000 \mathrm{kDa} ; 1.54)$ and ptFV $(163,000 \mathrm{kDa} ; 1.25)$, respectively ${ }^{14,26}$.

\subsubsection{Construction of FV Variants}

Constructs encoding for hFV comprising the ptFV Arg306 region (ptFV sequence Gly303-Thr309; Uniprot: Q7SZN0), designated pED-hFV-pt306, and ptFV comprising the human Arg306 region sequence (human full-length FV sequence Pro302-Leu308; Uniprot: P12259), named pED-ptFV-h306 were generated. Subsequently, ptFV's unique disulfide bond (ptFV residues Cys642 and Cys100216; Uniprot: Q7SZN0) was removed by replacing the cysteines for serine residues, thereby creating $p E D-$ ptFV-h306 S-S. ptFV-h306 and ptFV-h306 S-S were generated employing mutagenic complementary oligonucleotides as described ${ }^{14}$. The variant hFV-pt306 was generated using a pUC57 plasmid encoding pED-ptFV nucleotides 2124-2144, flanked by pED-hFV nucleotides, which was generated and purchased from Genscript (Piscataway, NJ, USA). The resulting pUC57 plasmid was digested with Xcml and Bsu36l, gel-purified, and subcloned into pED-hFV via digestion with the same enzymes.

\subsubsection{Expression and purification of FV variants}

Transfection of the plasmids encoding FV variants into baby hamster kidney cells, the selection of stable clones, and the expression and purification of $\mathrm{hFV}$ and ptFV were performed as described previously ${ }^{14}$, 26. Briefly, FV proteins were purified employing ion-exchange chromatography on a Q-Sepharose FF (hFV variants) or SP-Sepharose (ptFV variants) column (GE Healthcare) using a $\mathrm{NaCl}$ elution gradient in HBS supplemented with $5 \mathrm{mM} \mathrm{CaCl}_{2}$ and $1 \mathrm{mM}$ benzamidine (BZA), $\mathrm{pH}$ 7.4. Fractions containing FV activity were pooled and dialyzed against $20 \mathrm{mM}$ HEPES, $5 \mathrm{mM} \mathrm{CaCl}_{2}, \mathrm{pH} 7.4$ for human $\mathrm{FV}$ variants or $20 \mathrm{mM}$ MES, $5 \mathrm{mM} \mathrm{CaCl}$, pH 6.0 for ptFV variants, loaded on a POROS HQ/20 (hFV variants) or HS/20 column (ptFV variants) (Applied Biosystems) and eluted using a $\mathrm{NaCl}$ gradient. Protein purity was assessed by SDS-PAGE analysis using pre-cast $4 \%-12 \%$ gradient gels under nonreducing and reducing conditions (50mM DTT) using the MOPS buffer system (Life technologies; Carlsbad, CA, USA) followed by staining with Coomassie Brilliant Blue R-250. For pretreatment with thrombin, FV variants 
were incubated for 15 minutes with $\alpha$-thrombin (molar ratio: hFV 15:1; ptFV 3.75:1) in assay buffer at $37^{\circ} \mathrm{C}$, followed by the addition of a 10 -fold molar excess of hirudin over thrombin.

\subsubsection{Macromolecular substrate activation}

Steady-state initial velocities of macromolecular substrate cleavage were determined discontinuously at $25^{\circ} \mathrm{C}$ in assay buffer as described ${ }^{27,} 28$. Briefly, unless otherwise stated, progress curves of prothrombin were obtained by incubating PCPS $(50 \mu \mathrm{M})$, DAPA $(10 \mu \mathrm{M})$, and prothrombin $(1.4 \mu \mathrm{M})$ with the various recombinant $F V(a)$ species $(20 \mathrm{nM})$, and the reaction was initiated with $0.1-1 \mathrm{nM}$ of $F X a$ or ptFXa upon which the rate of prothrombin conversion was measured ${ }^{27}$.

\subsubsection{Activated protein C-mediated inactivation of FVa variants}

For APC-mediated proteolysis, FVa variants $(500 \mathrm{nM})$ were incubated at $37^{\circ} \mathrm{C}$ with $\mathrm{APC}(\mathrm{hFVa} 10 \mathrm{nM}$; ptFVa $750 \mathrm{nM})$ in the presence of PCPS $(50 \mu \mathrm{M})$. In the presence of protein S (100nM), ptFVa variants $(50 \mathrm{nM})$ were treated with $75 \mathrm{nM}$ APC. Activity assay mixtures contained prothrombin (1.4 $\mu \mathrm{M}), \mathrm{PCPS}$ $(50 \mu \mathrm{M})$, DAPA $(10 \mu \mathrm{M}), \mathrm{hFVa}(0.2 \mathrm{nM})$ or ptFVa variants $(0.1 \mathrm{nM})$, and FXa $(2 \mathrm{nM})$ or ptFXa $(1 \mathrm{nM})$, respectively. Prothrombin activation was determined as described under the section "Macromolecular substrate activation". Identification of the APC cleavage sites by $\mathrm{N}$-terminal sequence analysis was performed at The Protein Facility of the lowa State University Office of Biotechnology (Ames, IA).

\subsubsection{Size-exclusion chromatography of activated protein C-treated FVa variants}

FVa variants $(100-500 \mu \mathrm{g}$ ) were subjected to APC treatment (hFVa $154.2 \mathrm{nM}$; ptFVa $1000 \mathrm{nM}$ ) for 15 (hFVa) or $180 \mathrm{~min}$ (ptFVa) at $37^{\circ} \mathrm{C}$ in the presence of $50 \mu \mathrm{M}$ PCPS. Reactions were quenched by the addition of $50 \mu \mathrm{M}$ PPACK and $1 \mathrm{mM}$ BZA. A $10 \mathrm{~mm} \times 300 \mathrm{~mm}$ column containing Superdex 200 Increase (GE Healthcare) was equilibrated in assay buffer supplemented with $50 \mu \mathrm{M}$ PPACK and $1 \mathrm{mM} \mathrm{BZA}$. The 100-400 $\mu \mathrm{L}$ reaction mixture was then applied to the column and eluted with equilibration buffer at a flow rate of $0.25 \mathrm{~mL} \mathrm{~min}^{-1}$ at room temperature. Fractions $(0.25 \mathrm{~mL})$ were collected and analyzed by SDS-PAGE or Western Blot using a polyclonal antibody directed against human FV (Affinity biologicals, Ancaster, Canada) and the monoclonal antibody directed against the Asn307-Arg506 fragment (AHV5146; Haematologic Technologies, Essex Junction, VT) as described ${ }^{29}$.

\subsubsection{Molecular structure preparation}

For molecular dynamics (MD) simulation ${ }^{30}$, the 3D structures of $h F V a^{31}$ and ptFV (PDB ID: 4BXS) were used to build in missing loops with Loop Modeling suite in YASARA ${ }^{32}$. In addition, to simulate the experimentally used ptFV variant, the original region (303-GNPDTLT-309) was exchanged for the human Arg306 region (302-PKKTRNL-308), and the unique Cys642-Cys1002 disulfide bond was disrupted. To mimic the APC-cleaved protein structure, both human and P. textilis FV were separated into two protein fragments, reflecting their state after cleavage by APC at Arg306 and Arg506/Lys507. To expedite simulations, the $\mathrm{C} 1$ and $\mathrm{C} 2$ domain structures were excluded from simulation for both the human and $P$. textilis FV structures, given that these domains are structurally non-related with the A2 domain. 


\subsubsection{Molecular Dynamics}

Each simulation system was solvated with an explicit TIP3P water model and neutralized by addition of $\mathrm{Cl}$ - or $\mathrm{Na}+$ ions. The topology and coordinate files were prepared with the Amber ff14SB ${ }^{33}$ force field. During MD simulations, two initial energy minimization runs were performed using a steepest descent algorithm of 50,000 steps. Subsequently, each system was gradually heated from $0 \mathrm{~K}$ to $300 \mathrm{~K}$ within $50 \mathrm{ps}$. This step was followed by another 50 ps equilibration run with a constant temperature of $300 \mathrm{~K}$. After that, a $100 \mathrm{~ns}$ production run was carried out using the SHAKE algorithm ${ }^{34}$ to constrain bonds involving hydrogen atoms. The minimization, heating, equilibration, and production runs were all applied using AMBER $16^{35}$.

\subsubsection{Binding free energy calculations and per-residue free energy decomposition analysis}

From the 100 ns production run trajectory, 2000 frames were extracted between $t=0-20$ ns and $t=80$ $100 \mathrm{~ns}$, to calculate binding free energies. The Molecular Mechanics/Generalized Born Surface Area (MM/GBSA) method ${ }^{36}$ was successfully employed using igb $=5 \mathrm{~GB}$ model in AMBER, which can be briefly summarized by the following equations:

$$
\begin{gathered}
\Delta G_{\text {binding }}=G_{\text {complex }}-G_{\text {receptor }}-G_{\text {ligand }} \\
\Delta G_{M M / G B S A}=E_{\text {int }}+E_{\text {ele }}+E_{v d W}+E_{\text {polar }}+E_{\text {nonpolar }}-T \Delta S
\end{gathered}
$$

The binding free energy was calculated as the differences between free energy of the complex and the sum of the separate receptor and ligand shown in equation (1). The relative free energy $\triangle G M M / G B S A$ was estimated by the sum of every component in equation (2), with $E_{\text {int }}$, Eele and $E_{v d w}$ representing internal, electrostatic and van der Waals interactions; the solvation contribution is shown with $E_{\text {polar }}$ and

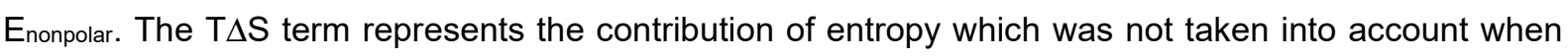
calculating relative binding free energies in our study. To determine key residues involved in binding, a per-residue energy contribution was calculated and analyzed based on the same factors used in above calculations.

\subsubsection{Statistical analysis}

All in vitro data are presented as mean \pm 1 standard deviation and are the result of at least two to three independent experiments, unless otherwise stated. 


\subsection{Results}

\subsubsection{Expression and characterization of FV variants}

Previously we reported that ptFV retains full cofactor activity despite APC-catalyzed proteolysis ${ }^{11,} 14$. APC cleaves the ptFV A2 domain at Lys507 and Arg742, homologous to human Arg506 and Arg709, respectively. Intriguingly, sequence analysis revealed the lack of a potential Arg306 cleavage site and a non-conserved sequence consisting of ptFV residues 303-GNPDTLT-309 (Figure 1A). As the corresponding region is strictly conserved in mammals, we exchanged the non-conserved ptFV region with the human Arg306 region (302-PKKTRNL-308) of constitutively active B-domainless hFV (Figure 1B) to generate the chimeric variants hFV-pt306 and ptFV-h306 (Figure 1C). Since a unique disulfidebond in ptFV covalently links the $\mathrm{A} 2$ and $\mathrm{A} 3$ domains $^{16}$, this structural element could potentially prevent $\mathrm{A} 2$ domain dissociation and ptFVa inactivation. To exclude the contribution of this disulfide bond to $\mathrm{ptFVa}$ inactivation, we generated an additional variant based on ptFV-h306 in which the cysteines involved were substituted by serines, generating ptFV-h306 S-S (Figure 1C). The FV variants were stably expressed in BHK cells and purified to homogeneity by ion-exchange chromatography. Each thrombin-activated FVa variant migrated on SDS-PAGE similar to recombinant wild-type hFVa or ptFVa (Figure 1D). In addition, SDS-PAGE analysis confirmed that the unique disulfide bond was preserved in ptFV-h306 but eliminated in ptFV-h306 S-S. Functional assessment of each FV variant revealed a similar apparent affinity for their respective FXa species (Table S1), indicating that substitution of the Arg306 region does not interfere with cofactor-FXa assembly.

A H. sapiens KNCPKKTRNLKK I T

B. taurus KNCAKKTRNPKKLT

M. musculus KNCPKKTRSPKTLT

S. scrofa KNC AKKTRKPKKLT

P. troglodytes KNCPKKTRNLKKI T

P. textilis-liver KDCGN PDT LTRKLS

P. textilis-venom KDCGN PDT LTRKLS

B

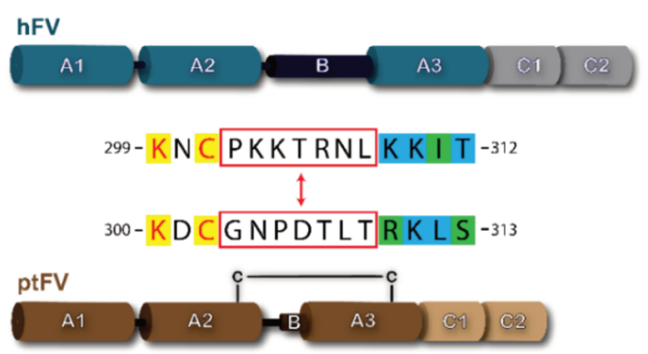

C

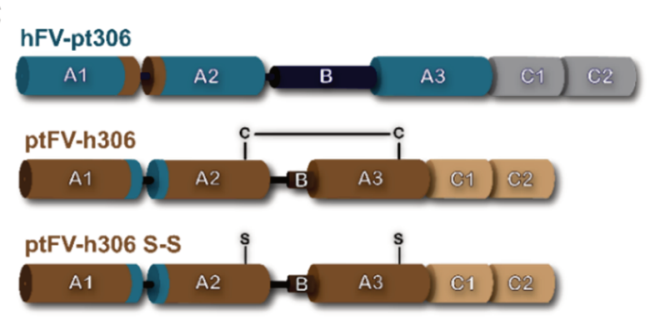

D

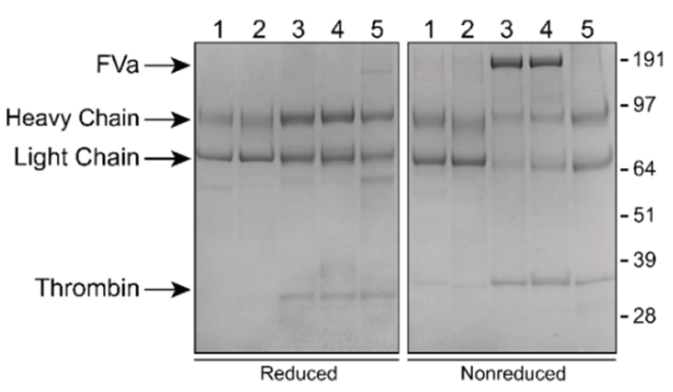

Figure 1. Generation and SDS-PAGE analysis of purified factor Va variants. (A) Sequence alignment of the Arg306 region. Completely conserved residues are highlighted in yellow, partly conserved residues in blue, and residues belonging to the same classification are indicated in green. (B) Schematic representation of $B$ domain truncated human FV (hFV) and venom-derived $P$. textilis FV (ptFV). The Arg306 region indicated by the red boxes was exchanged between $h F V$ and ptFV. (C) Schematic representation of generated FV variants. Human regions are indicated in blue/grey, ptFV regions are brown/beige. Swapping the Arg306 region generated hFV-pt306 and ptFVh306. Additional removal of the disulfide bond by replacing the cysteines (Cys642 and Cys 1002) for serines in ptFV generated ptFV-h306 S-S. (D) SDS-PAGE of purified thrombin-activated FVa variants (2 $\mu g$ per lane) under reducing (left panel) and non-reducing (right panel) conditions visualized by staining with Coomassie Brilliant Blue R-250. Lane 1, hFVa; lane 2, hFVa-pt306; lane 3, ptFVa; lane 4, ptFVa-h306; lane 5, ptFVa-h306 S-S. Relevant fragments and the apparent molecular weights of the standards are indicated. 


\subsection{2 ptFVa variants maintain cofactor function upon proteolysis by APC}

We next assessed APC-mediated proteolysis of the ptFVa variants comprising the two major APC cleavage sites that are essential for the inactivation of mammalian $\mathrm{FVa}^{21}, 24$. Thrombin-activated FVa variants were subjected to APC and the resulting cleavage pattern was analyzed by SDS-PAGE. While in hFVa variants full proteolysis was achieved in 15 min by $10 \mathrm{nM} \mathrm{APC}$, high concentrations of APC $(750 \mathrm{nM})$ were required for significant proteolysis of the ptFVa variants. As previously described ${ }^{14}$, ptFVa was cleaved at Lys507 (Figure S1A), which is equivalent to human Arg506. Conversely, we observed additional protein bands consistent with the cleavage of Arg306 in ptFVa-h306 (Figure 2A). N-terminal sequencing of the cleavage products confirmed the generation of a ptFVa variant comprising the homologous Arg306 and Arg506 cleavage sites (Figure S2). Removal of the unique disulfide bond in ptFVa-h306 S-S did not affect the cleavage pattern relative to ptFVa-h306 (Figure S1B). Moreover, while hFVa was proteolyzed at Arg306, Arg506, and Arg679 (Figure S1C), an Arg306-like cleavage was absent in hFVa-pt306 (Figure 2B), demonstrating that the non-conserved ptFV region is not targeted by APC.

Subsequently, the cofactor activity of the APC-proteolyzed FVa variants was assessed. Whereas hFVa was fully inactivated within 5 minutes, functional analysis employing a purified prothrombinase assay revealed partial inactivation of hFVa-pt306 by APC, in a manner similar to the previously characterized hFVa-Arg306Thr variant ${ }^{37}$ (Figure 2C). Surprisingly, despite introduction of the Arg306 cleavage site and removal of the unique disulfide bond, both chimeric ptFVa variants maintained full cofactor activity during APC-treatment (Figure 2D). In addition, assessment of the ptFVa cofactor activity in the presence of protein $\mathrm{S}$, a cofactor for $\mathrm{APC}^{38,39}$, revealed identical results (Figure 2E). These findings indicate that the absence of a Arg306-like cleavage site and presence of ptFVa's unique disulfide bond do not contribute to the molecular mechanism that is at the basis of ptFVa's functional APC-resistance. 
A

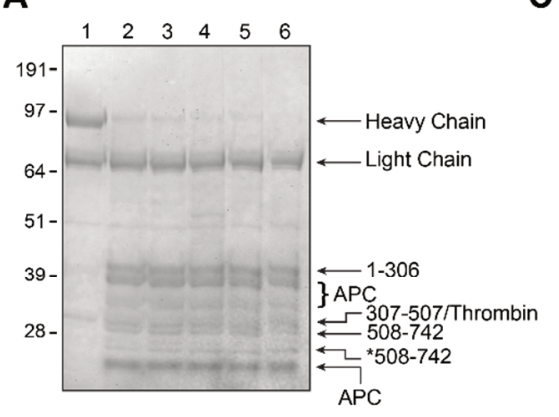

B

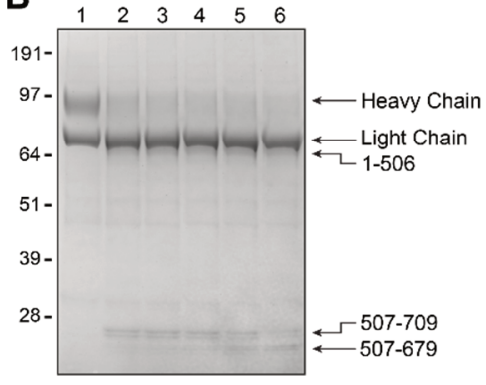

C

D
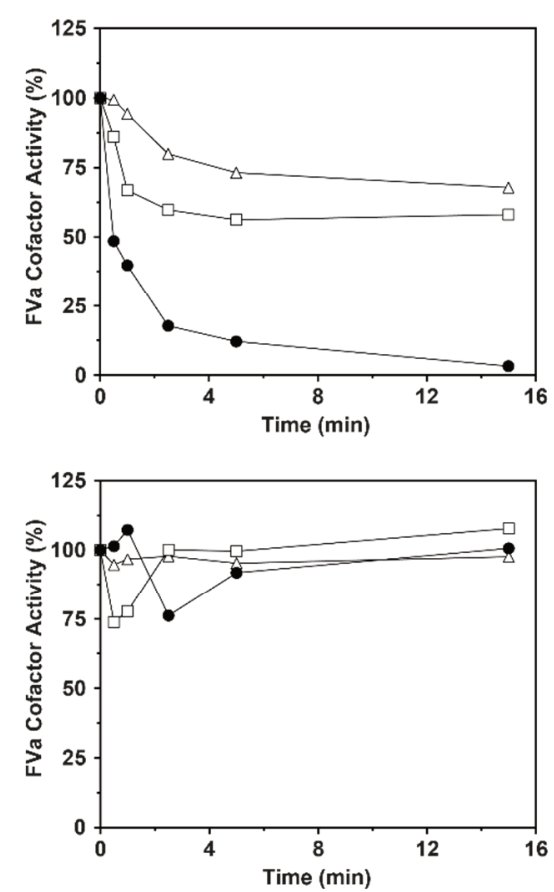

E

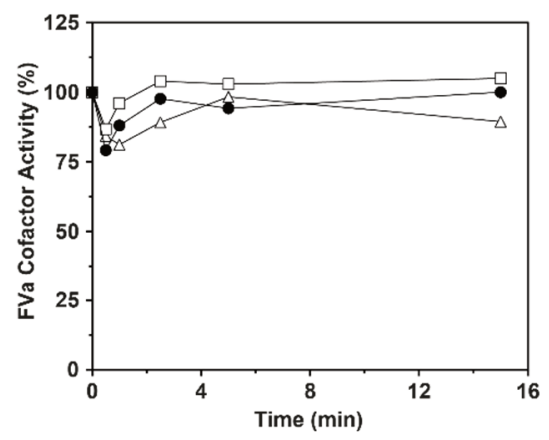

Figure 2. Activated protein C-mediated proteolysis of human and P. textilis factor Va variants. (A-B) SDS-PAGE of activated protein C (APC)-treated ptFVa-h306 (A) and hFVa-pt306 (B) (3 $\mu$ g per lane) under reducing conditions and visualized by staining with Coomassie Brilliant Blue R-250. Lanes 1-6 represent time samples quenched at 0 , $0.5,1,2.5,5$, and $15 \mathrm{~min}$. Relevant FVa fragments and the apparent molecular weights of the standards are indicated. We hypothesize that *508-742 is cleaved at a C-terminal position because its $\mathrm{N}$-terminal sequence was determined to be the same as 508-742 (see Supplementary Figure 2). (C) Reaction mixtures containing $50 \mu \mathrm{M}$ PCPS and $500 \mathrm{hM} \mathrm{hFVa} \mathrm{(circles),} \mathrm{hFVa-pt306} \mathrm{(squares),} \mathrm{or} \mathrm{hFVa-Arg306Thr} \mathrm{(triangles)} \mathrm{were} \mathrm{incubated} \mathrm{with} 10$ nM APC and (D-E) similar mixtures containing $500 \mathrm{nM}$ ptFVa (circles), ptFVa-h306 (squares) or ptFVa-h306 S-S (triangles) were incubated with $750 \mathrm{nM} A P C$ in the absence $(D)$ or presence $(E)$ of $100 \mathrm{nM}$ protein S. At selected time intervals, samples were removed for cofactor activity by determining the initial velocity of prothrombin conversion. The data are representative of three independent experiments.

\subsection{3 ptFV retains structural integrity following APC-mediated proteolysis}

APC-catalyzed proteolysis at the two major FVa cleavage sites Arg306 and Arg506 is known to result in A2 domain dissociation ${ }^{24}$. Since our ptFVa variants maintain full cofactor activity following APCtreatment, we hypothesized that despite selective proteolysis, A2 domain dissociation is prevented. Initial observations employing native-PAGE analysis suggested that APC-mediated proteolysis resulted in the fragmentation of $\mathrm{hFV}$, whereas ptFV and ptFV-h306 S-S migrated as single molecules (Figure S3). To explore the structural consequences of proteolysis in more detail we determined the structural integrity of the APC-treated ptFVa variants by size-exclusion chromatography. Following APC treatment, the proteolyzed FVa variants were applied to a $10 \times 300 \mathrm{~mm}$ Superdex 200 Increase column and fractions were collected and analyzed by SDS-PAGE or Western blotting. In accordance with the nativePAGE, size-exclusion profiles suggested the fragmentation of APC-treated hFVa (Figure 3A), whereas a single elution peak was observed for APC-cleaved ptFVa (Figure 3B). The elution profile of APCtreated ptFVa-h306 S-S was comparable to that of ptFVa following APC treatment (Figure 3C), although an additional elution peak was observed as a result of impurities (Figure 3F). As expected from previous observations $^{24}$, the two hFVa A2 domain fragments Asn307-Arg506 and Gly507-Arg679 migrated separately from the rest of the molecule (A1-A3-C1-C2 domains) (Figure 3D). Conversely, ptFVa eluted 
as a single complex (Figure 3E), corroborating our functional data. Remarkably, despite introduction of the Arg306 region and deletion of the unique disulfide bond, ptFVa-h306 S-S retained structural integrity following proteolysis by APC (Figure 3F). As such, these results demonstrate that the dissociation of the ptFVa A2 domain is prevented by non-covalent interactions. Functional analysis of the chimeric $\mathrm{FVa}$ variants under conditions of high temperatures (Figures S4A-B) or high ionic strength (Figure S4C) did not reveal significant differences relative to wild-type FVa, suggestive of an enhanced A2 domain binding stability.

A

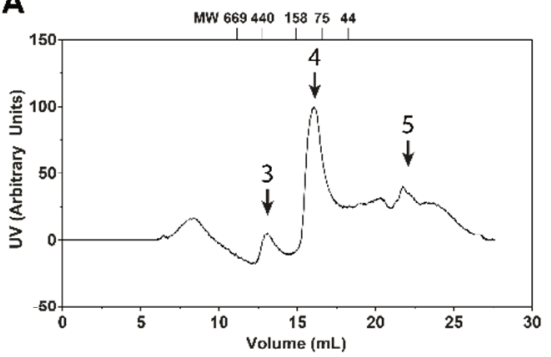

D

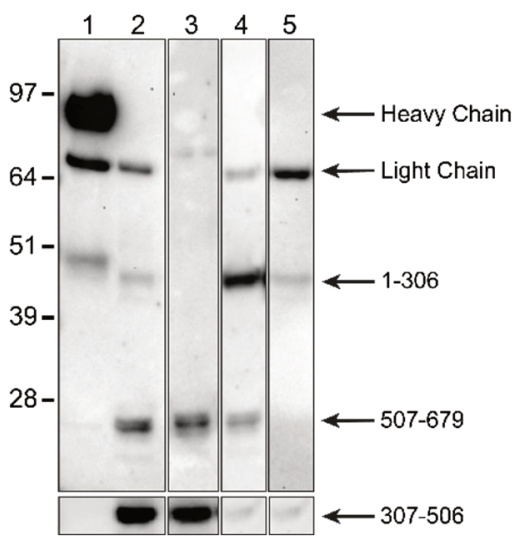

B
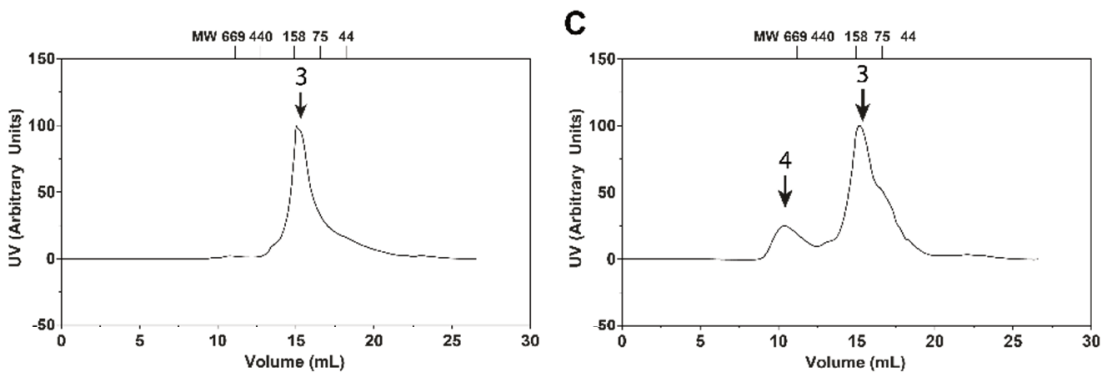

$\mathbf{E}$

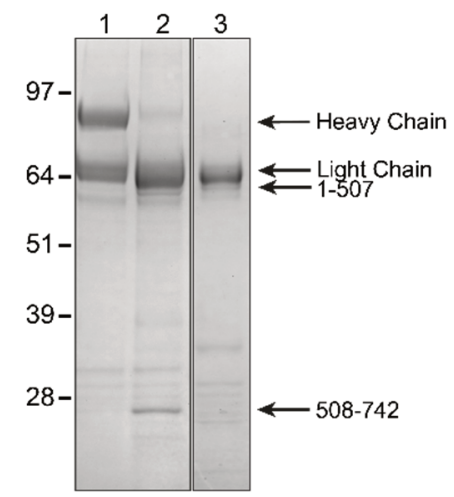

$\mathbf{F}$

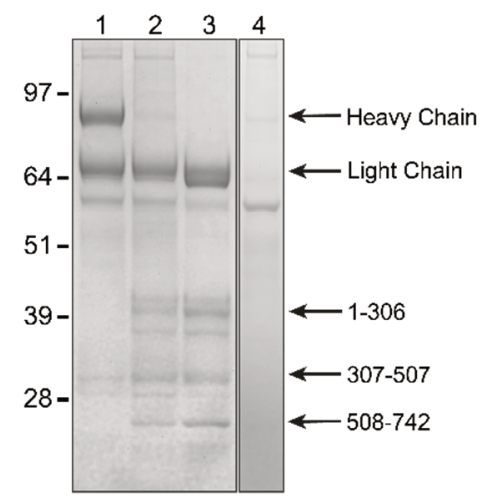

Figure 3. Size-exclusion chromatography of activated protein C-treated FVa variants. (A-C) Size-exclusion elution profiles of APC-treated hFVa (A), ptFVa (B), or ptFVa-h306 S-S (C) performed as detailed in 'Materials and Methods'. Arrows and numbers indicate the respective fractions shown in D-F. MW, molecular weight. (D) Western blot analysis of $h F V a$ under reducing conditions following proteolysis by APC and size-exclusion chromatography elution. Lane 1, thrombin-activated hFVa; lane 2, APC-treated hFVa; lane 3, A2 domain fraction; lane 4, A1-A3-C1$C 2$ and $A 2$ domain fraction; lane 5, A1-A3-C1-C2 domains fraction. (E-F) SDS-PAGE of APC-treated ptFVa (E) or ptFVa-h306 S-S (F) following size-exclusion chromatography under reducing conditions and visualized by staining with Coomassie Brilliant Blue R-250. Lanes 1, thrombin-activated ptFVa variant; lanes 2, APC-treated ptFVa variant; lanes 3 and 4, elution fractions indicated by the black arrows and numbers in B-C. Relevant FVa fragments and the apparent molecular weights of the standards are indicated. The data are representative of two independent experiments. Panels D-F are composed of two Western Blots or SDS-PAGE gels, respectively.

\subsubsection{Stable binding free energy for the A1-A2-A3 domains of APC-proteolyzed ptFVa-h306 S- $S$ during MD simulations}

In order to gain more insight into the structural stability of human and $P$. textilis FV, 100ns MD simulations were performed on hFVa and ptFVa-h306 S-S. The model of ptFVa-h306 S-S was generated from the X-ray structure of ptFV (PDB ID: 4BXS) in which the non-conserved region was 
substituted for the corresponding human Arg306 region and the Cys642-Cys1002 disulfide bond was disrupted. In addition, the peptide bonds that resemble Arg306 and Arg506/Lys507 cleavage were hydrolyzed in both FVa models to simulate an APC-proteolyzed state, and the C1-C2 domains were excluded from the MD simulations. Calculation of the free energies for the interaction between the $A 2$ and A1-A3 domains revealed that these were comparable for hFVa and ptFVa-h306 S-S during the initial trajectory of the simulation ( $\mathrm{t}=0-20 \mathrm{~ns}$ ), with a binding free energy of $-76 \pm 25 \mathrm{kcal} / \mathrm{mol}$ for hFVa and $-76 \pm 14 \mathrm{kcal} / \mathrm{mol}$ for ptFVa-h306 S-S (Table 1). However, in the final part of the MD trajectory ( $\mathrm{t}=80-100 \mathrm{~ns})$, the binding free energy was remarkably increased to $-39 \pm 16 \mathrm{kcal} / \mathrm{mol}$ for $\mathrm{hFVa}$, whereas a substantial decrease to $-90 \pm 14 \mathrm{kcal} / \mathrm{mol}$ was observed for ptFVa-h306 S-S. This suggests that over the course of the MD simulation, hFVa lost important interactions that facilitate the binding of the A2 to

Table 1. The binding free energies of the A2 and A1-A3 domain interaction. Binding free energies were calculated using the MD trajectory from $t=0-20 n s$ or $t=80-100 n s$ as described in 'Materials and Methods'. N.A., For $h F V a-$ ptloop2 the $t=0$-20ns trajectory was excluded from the analysis due to the initial destabilization of the structure following loop exchange. Energies are expressed as $\mathrm{kcal} / \mathrm{mol} \pm S . D$.

\begin{tabular}{cccc}
\hline MD trajectory & $\begin{array}{c}\mathrm{hFVa} \\
(\mathrm{kcal} / \mathrm{mol})\end{array}$ & $\begin{array}{c}\mathrm{ptFVa}-\mathrm{h} 306 \mathrm{~S}-\mathrm{S} \\
(\mathrm{kcal} / \mathrm{mol})\end{array}$ & $\begin{array}{c}\mathrm{hFVa-ptloop2} \\
(\mathrm{kcal} / \mathrm{mol})\end{array}$ \\
\hline $0-20 \mathrm{~ns}$ & $-75.46 \pm 24.99$ & $-75.58 \pm 13.68$ & N.A. \\
$80-100 \mathrm{~ns}$ & $-39.36 \pm 15.57$ & $-90.47 \pm 13.51$ & $-63.53 \pm 15.89$ \\
\hline
\end{tabular}

the A1-A3 domains. To examine these interdomain interactions in more detail, the binding free energies between the $\mathrm{A} 2$ and $\mathrm{A} 1$ or $\mathrm{A} 2$ and $\mathrm{A} 3$ domains were individually assessed. Interestingly, a loss of interaction between the $A 2$ and $A 1$ domains in hFVa was observed for the $t=80-100$ ns interval ( $4 \pm 10 \mathrm{kcal} / \mathrm{mol}$, Table 2 ), while the binding free energy characterizing the A2-A3 interaction was nearly identical for both MD trajectories monitored. In contrast, the binding free energy between the A2-A1 or A2-A3 domains in ptFVa-h306 S-S remained unaffected during the simulations, indicative of a stable interaction. As such, these data confirm our in vitro studies by indicating that the structural integrity of the A1-A2-A3 domains in P. textilis FV is maintained following APC cleavage.

Table 2. Binding free energies for the binding of the A2 domain to the A1 or A3 domain. Binding free energies were calculated using the MD trajectory from $t=0-20 n s$ or $t=80-100$ ns as described in 'Materials and Methods'. Energies are expressed as $\mathrm{kcal} / \mathrm{mol} \pm$ S.D.

\begin{tabular}{cccc}
\hline MD trajectory & Domain & $\begin{array}{c}\mathrm{hFVa} \\
(\mathrm{kcal} / \mathrm{mol})\end{array}$ & $\begin{array}{c}\mathrm{ptFVa} \text {-h306 S-S } \\
(\mathrm{kcal} / \mathrm{mol})\end{array}$ \\
\hline \multirow{2}{*}{$0-20 \mathrm{~ns}$} & A2-A1 & $-37.20 \pm 15.69$ & $-41.37 \pm 9.71$ \\
& A2-A3 & $-43.45 \pm 13.41$ & $-41.55 \pm 9.20$ \\
\hline \multirow{2}{*}{$80-100 \mathrm{~ns}$} & A2-A1 & $4.37 \pm 10.39$ & $-36.44 \pm 8.98$ \\
& A2-A3 & $-46.69 \pm 10.40$ & $-55.39 \pm 10.12$ \\
\hline
\end{tabular}




\subsubsection{Stable hydrogen bond pairs between the $A 2$ and $A 1-A 3$ domains preserve $A 2$ domain association in P. textilis FV}

To further uncover the molecular mechanisms that are at the basis of the differential A2-A1 domain interactions observed for $\mathrm{hFV}$ and $\mathrm{ptFV}$, the hydrogen bonds that contribute to the overall interdomain binding affinities were assessed. In accordance with the loss of A2-A1 domain interaction, the number of hydrogen bonds between the surfaces of the $A 2$ and $A 1$ domain in hFVa reduced over time during the MD simulations (Figure 4A). Representative structural snapshots revealed significant conformational changes in the $\mathrm{A} 2$ domain, suggesting that the $\mathrm{A} 1$ domain interacting regions are highly

A

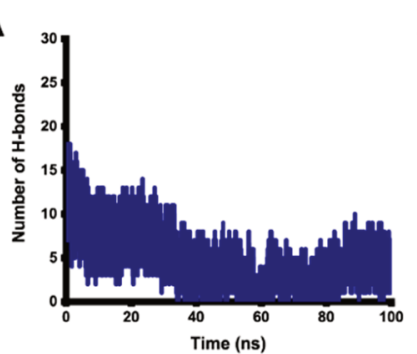

D

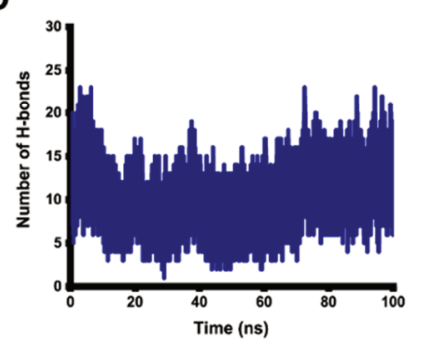

G

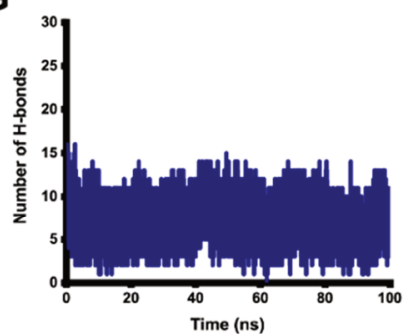

J

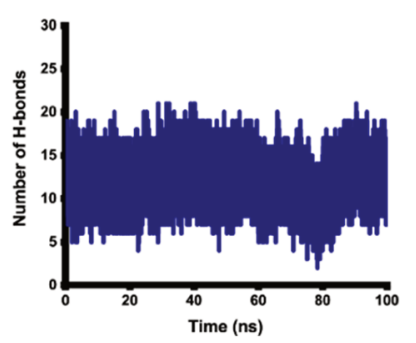

B

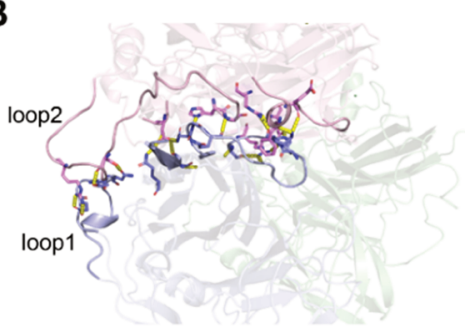

E

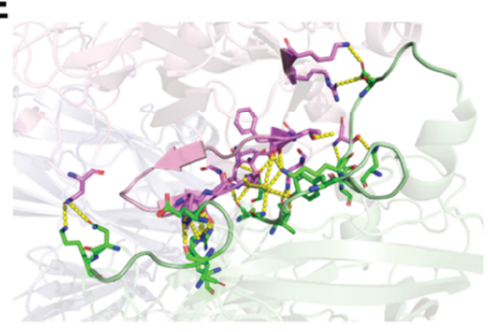

H

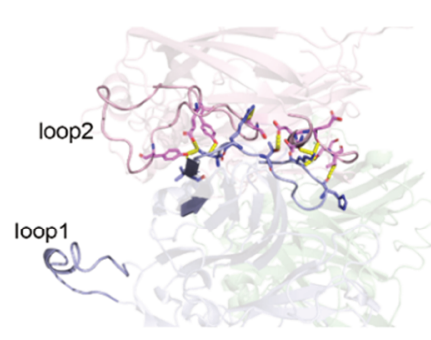

K

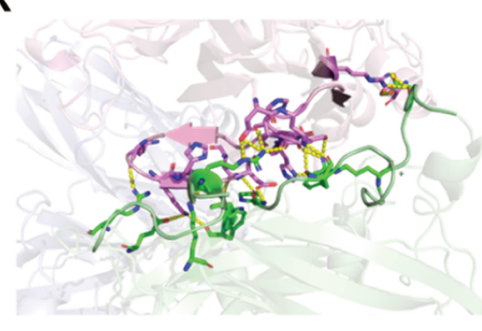

C

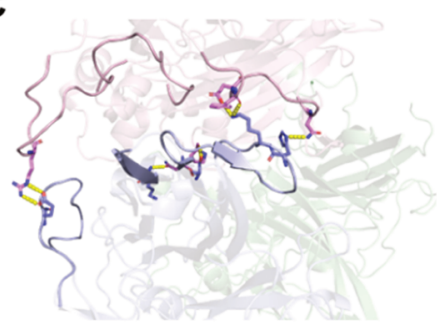

F

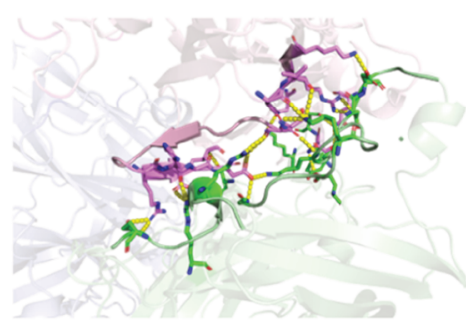

I

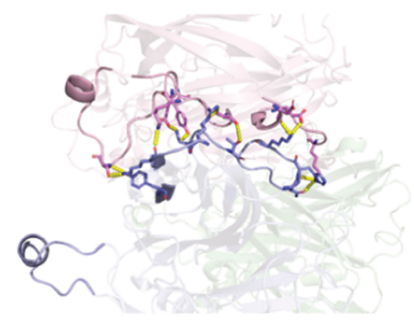

L

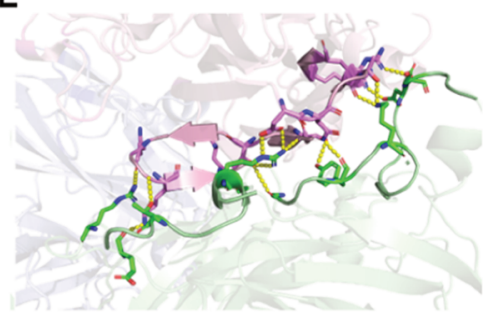

Figure 4. Comparison of interdomain hydrogen bonds between surfaces of the $A 2$ and the $A 1 / A 3$ domains in human and $P$. textilis $F V$. The structural conformation and interdomain hydrogen bonds between the surfaces of the A2 and $A 1(B-C, H-I)$ or $A 2$ and $A 3(E-F, K-L)$ domains are shown at the start (t=Ons) (B, E, H, K) and end ( $t=100$ ns) (C, F, I, L) of the $h F V a(B-C, E-F)$ and ptFVa-h306 S-S (H-I, K-L) MD simulations. The A1, A2, and A3 domains are indicated in blue, pink, and green, respectively. Residues that engage in hydrogen bond formation are shown in stick configuration. Hydrogen bonds are indicated by the yellow dashed lines. The A1 domain loop1 and A2 domain loop2 represent the identified unique loop conformations. (A, $D, G, J)$ Interdomain hydrogen bonds between the $A 2$ and $A 1(A, G)$ or $A 2$ and $A 3(D, J)$ domains were quantified during a 100ns $M D$ simulation of $h F V a(A, D)$ and ptFVah306 S-S (G, J). 
flexible following APC-catalyzed proteolysis (Figures 4B-C). In contrast, the number of hydrogen bonds formed between the $A 2$ and $A 3$ domains remained in a dynamic equilibrium during the simulation (Figures 4D-F), corroborating the stable A2-A3 binding affinity. The hydrogen bond formation between the A2-A1 and A2-A3 domains in APC-proteolyzed ptFVa-h306 S-S appeared to be balanced and most hydrogen bonds persisted during the simulation (Figures 4G-L). These stable interactions might be explained by the conformational constraints of the A2 domain, since only minor structural changes in the binding surfaces were observed between structures at different timepoints. Identification of residues involved in key hydrogen bond interactions during $>20 \%$ of the MD simulations demonstrated ptFVa-h306 S-S to accommodate a larger fraction of these interactions relative to hFVa (Table S2 and Figure S5). Collectively, these findings indicate that $\mathrm{ptFV}$ comprises a high number of A2-A1 interactions that are preserved upon APC cleavage.

\subsubsection{A unique loop conformation in the A2 domain prevents A2 domain dissociation in $P$. textilis FV}

Structural comparison of the MD simulations revealed that the A2 domain of hFVa and ptFVa-h306 S$S$ adopt strikingly different conformations (Figure 4). In hFVa, we observed a major conformational change in a surface-exposed A2 domain loop (loop2) that is extended into the solvent and interacts with an A1 domain loop (loop1) via one stable interaction pair (Glu20-Arg439; Figures 4, S5A). While human and $P$. textilis $\mathrm{FVa}$ share $55 \%$ sequence identity, the positioning of loop1 and loop2 is conserved (Figure S6). Interestingly, MD simulations in ptFVa-h306 S-S indicated that loop2 is more compact and does not interact with loop1 (Figures 4, S5B). Supporting the structural observations, assessment of the solvent accessible surface area (SASA) of individual A domains or A domain complexes over the MD trajectories further revealed that the SASAs of ptFVa-h306 S-S were smaller relative to hFVa (Figure 5). To specifically determine the role of loop2 in A2 domain stability, we created an in silico chimeric $\mathrm{hFV}$ a structure in which the human loop2 was exchanged for the homologous ptFV loop2 region (hFVaptloop2), upon which a 100ns MD simulation was performed (Figure 6A). As substitution of loop2 destabilized the structure and required several nanoseconds to adopt a stable state, we excluded the initial 20ns from our analysis. Intriguingly, the A2 domain binding free energy in hFVa-ptloop2 was markedly decreased relative to $\mathrm{hFVa}$ (from $-39 \pm 16 \mathrm{kcal} / \mathrm{mol}$ to $-64 \pm 16 \mathrm{kcal} / \mathrm{mol}$ ), although it remained higher compared to ptFVa-h306 S-S (-90 $\pm 14 \mathrm{kcal} / \mathrm{mol}$ ) (Table 1). In similar fashion, exchange of loop2 substantially decreased the average A2 domain SASA ( $\left.1.58 \times 10^{4} \AA^{2} \pm 0.05 \AA^{2}\right)$ as compared to hFVa $\left(1.67 \times 10^{4} \AA^{2} \pm 0.04 \AA^{2}\right)$, but remained larger relative to ptFVa-h306 S-S $\left(1.48 \times 10^{4} \AA^{2} \pm 0.04 \AA^{2}\right)$, illustrating the compact conformation of the ptFV loop2. Consistent with these findings, the A2-A1 domain hydrogen bonds were stabilized in chimeric hFVa-ptloop2, while the exchange of loop2 did not affect the interactions between the A2 and A3 domains (Figures 6B-D). Nonetheless, we cannot exclude that differences in hydrogen bond pairing between the $\mathrm{A} 2$ and $\mathrm{A} 3$ domains contribute to the decreased binding free energy for A2 domain binding in hFVa-ptloop2. Collectively, this structural bioinformatics analysis confirms that the compact and inflexible ptFV loop2 stabilizes the surface interactions between the $\mathrm{A} 2$ and $\mathrm{A} 1$ domains following APC proteolysis and could provide a mechanism that prevents $\mathrm{A} 2$ domain dissociation in ptFVa. 


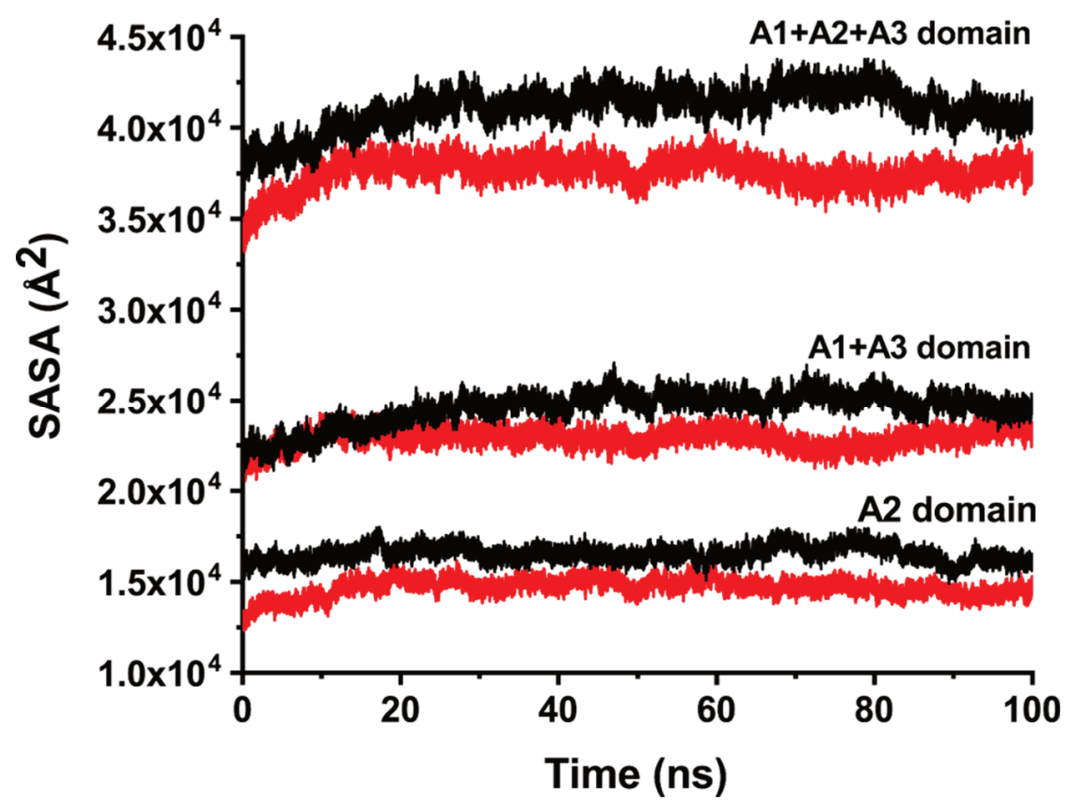

Figure 5. Solvent accessible surface areas (SASA) in human and P. textilis FV. The SASA of the A2 domain, the $A 1$ and $A 3$ domain complex, and the A1, A2, and A3 domain complex were calculated as described in 'Materials and Methods'. The black and red lines represent human and $P$. textilis FV, respectively.

A

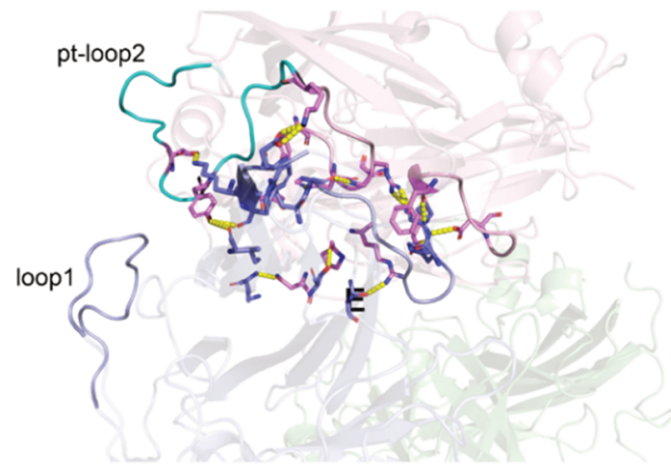

C

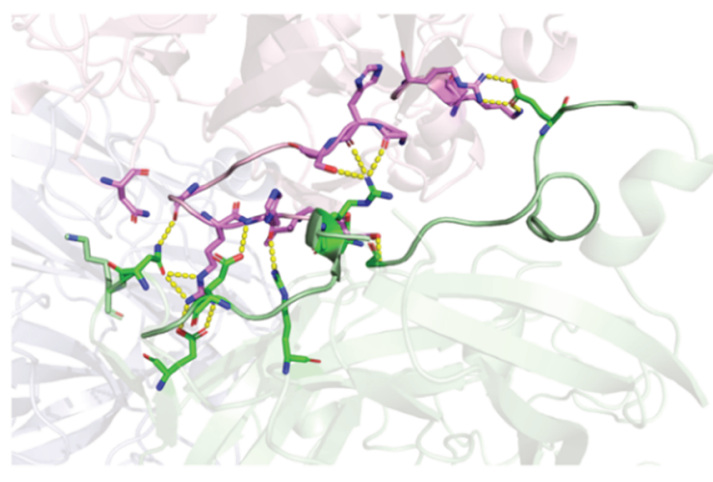

B
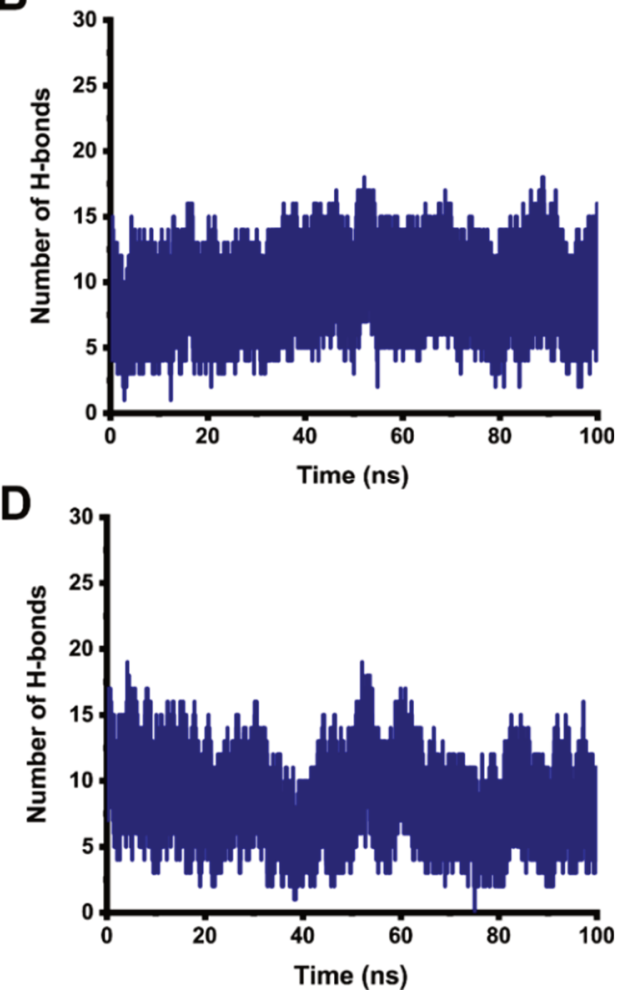

Figure 6. Stable hydrogen bond formation between the A2 and A1 domains upon substitution of human loop2 for the corresponding region in P. textilis FV. In silico exchange of loop2 in hFVa for the homologous loop2 in ptFV generated a chimeric $h F V a$ variant. $(A, C)$ Interdomain hydrogen bonds between the surfaces of the $A 2$ and $A 1(A)$ or $A 2$ and $A 3(C)$ domains are shown in a representative snapshot of the 100ns MD simulation of chimeric $h F V a$. The A1, A2, and A3 domains are indicated in blue, pink, and green, respectively. Residues that engage in hydrogen bond formation are shown in stick configuration. Hydrogen bonds are indicated by the yellow dashed lines. $(B, D)$ Interdomain hydrogen bonds between the $A 2$ and $A 1(B)$ or $A 2$ and $A 3(D)$ domains were quantified during a 100ns MD simulation of the chimeric hFVa model. 


\subsection{Discussion}

One of the essential characteristics underlying any hemostatic response is the timely inactivation of proteases and cofactors following proteolytic activation of their respective substrate. The specialized mechanisms of the anticoagulant system thereby prevent uncontrolled blood clotting and life-threatening thrombotic events. Our work, amongst others ${ }^{11}, 14,18$, highlights the importance of strict regulatory pathways for $\mathrm{FV}$ and shows that bypassing these mechanisms can be used as a potent weapon to gain evolutionary advantages.

We previously speculated that absence of the Arg306 cleavage site, required for the inactivation of mammalian $\mathrm{FVa}$, is at the basis of the functional APC-resistance in $\mathrm{ptFVa}^{14,18}$. To test this hypothesis, chimeric ptFV variants were generated that lack the unique A2-A3 domain disulfide bond and/or comprise the human Arg306 region. Our functional assessments show that despite Arg306 cleavage and disruption of the unique disulfide bond, ptFVa retains cofactor activity upon APC-catalyzed proteolysis. As such, our findings demonstrate that these elements in ptFV do not play a role in the functional resistance to APC.

Liver-derived ptFV shares $96 \%$ sequence identity to its venom paralog ${ }^{40}$ and we previously revealed that liver-expressed ptFV shares the procoagulant characteristics of venom-derived ptFV, including the functional resistance to $A P C^{18}$. These observations raise fundamental questions on the hemostatic regulatory mechanisms in $P$. textilis. It is currently unknown whether Elapidae express protein $\mathrm{C}$, but if so, it would be of interest to characterize the mechanistic principles that hallmark the anticoagulant response in these snakes. It is also important to note that Arg306 is only conserved in mammals, whereas lower vertebrates do not seem to comprise a homologous Arg306 cleavage site. This indicates that lower vertebrates have a distinct mechanism to inactivate FVa.

Using size-exclusion chromatography, we showed that despite APC-mediated proteolysis A2 domain dissociation in ptFVa is prevented. Remarkably, the A2 domain remained associated following introduction of the Arg306 cleavage site in ptFVa. Attempts to weaken the non-covalent structural integrity of the APC-treated ptFVa variants failed to significantly impact their ability to function as cofactor for $\mathrm{ptFXa}$. These results demonstrate that following selective proteolysis ptFVa maintains structural integrity mediated by strong non-covalent interactions. Strikingly, these findings contradict our current understanding of $\mathrm{FVa}$ inactivation ${ }^{41}$ and imply the presence of unique structural elements that prevent A2 domain dissociation.

In an attempt to unravel the mechanistic principles of $\mathrm{A} 2$ domain association, we identified a crucial role for A1-A2 domain interactions. In hFVa, the binding free energy between the $\mathrm{A} 1$ and $\mathrm{A} 2$ domain increases drastically upon APC-mediated proteolysis. As a consequence, the interaction between the $\mathrm{A} 1$ and $\mathrm{A} 2$ domain is lost which seemingly triggers the dissociation of the A2 domain. The loss of A1A2 domain binding affinity coincides with a major conformational change in the hFVa loop2. This highly flexible loop engages in weak interactions with the A1 domain loop1 and facilitates several A1-A2 
domain hydrogen bonds. Upon APC-catalyzed proteolysis, the number of hydrogen bonds between the surfaces of the $A 2$ and $A 1$ domains reduces as a result of the conformational changes in the $A 2$ domain. Conversely, the A1-A2 domain binding free energy remains stable following APC-catalyzed cleavage of $\mathrm{ptFVa}$. In contrast to $\mathrm{hFVa}$, the ptFVa loop2 does not interact with loop1 and adopts a rigid conformation that is stabilized by several key hydrogen bonds. Exchange of the ptFV loop2 to hFVa significantly improved the binding affinity between the $\mathrm{A} 2$ and $\mathrm{A} 1-\mathrm{A} 3$ domains, providing further support for the central role of loop2 in the A2 domain association. Interestingly, loop2 is located directly distal from the Arg306 cleavage site, which may suggest that the stable non-covalent interactions are able to compensate for Arg306 cleavage.

Characterization of the mechanistic principles and identification of key regions or residues responsible for ptFV's functional resistance to APC could provide unique opportunities for the generation of novel stable FV or factor VIII (FVIII) variants for bleeding disorders such as hemophilia. Upon activation, the A2 domain of FVIIla dissociates spontaneously, markedly impacting the overall efficacy of FVIII products ${ }^{42,43}$. Moreover, the treatment of hemophilia A is complicated by the relative short half-life of $\mathrm{FVIII}^{44}$. In order to generate longer acting and more convenient treatment options, modifications of FVIII aiming to generate more stable proteins with an extended half-life have been the focus of product development ${ }^{45}$. One of such extended half-life products is a FVIII variant (Lonoctocog alfa) comprising a truncated B domain that covalently links the heavy and light chains. This single-chain FVIII exhibits an improved intrinsic stability and a markedly increased affinity to von Willebrand factor resulting in a prolonged half-life ${ }^{46,47}$. As an alternative strategy, the mechanism by which A2 domain dissociation is prevented in ptFVa could potentially be translated to FVIII considering the structural homology between FV and FVIII.

In conclusion, our findings highlight the exceptional structural features that have converted ptFV into an extremely procoagulant protein. Here we demonstrated that $\mathrm{ptFVa}$ maintains functional resistance to APC despite introduction of the Arg306 cleavage site, in sharp contrast to the APC response of mammalian FVa. To do so, ptFV has uniquely adapted non-covalent interactive regions in the $\mathrm{A} 1$ and A2 domains that stabilize the A2 domain following proteolysis by APC. Taken together, our findings have identified a unique structural element by which ptFV circumvents the regulatory mechanisms and shed new insights on the mechanistic principles of A2 domain dissociation and FVa inactivation.

\subsection{Acknowledgements}

This work was financially supported by the Bayer Hemophilia Awards Program (Special Project Award), Landsteiner Foundation for Blood Transfusion (LSBR, grant. no. 1451), and the China Scholarship Council (to X.L.). The funding agencies had no role in the preparation, review, or approval of the manuscript. 


\section{References}

1. Owren, P. A., Parahaemophilia; haemorrhagic diathesis due to absence of a previously unknown clotting factor. Lancet 1947, 1 (6449), 446-8.

2. Camire, R. M.; Bos, M. H., The molecular basis of factor V and VIII procofactor activation. J Thromb Haemost 2009, 7 (12), 1951-61.

3. Bos, M. H.; Camire, R. M., A bipartite autoinhibitory region within the B-domain suppresses function in factor V. J Biol Chem 2012, 287 (31), 26342-51.

4. Schreuder, M., et al., Blood coagulation factor Va's key interactive residues and regions for prothrombinase assembly and prothrombin binding. J Thromb Haemost 2019, 17 (8), 1229-1239.

5. Mann, K. G., et al., Surface-dependent reactions of the vitamin K-dependent enzyme complexes. Blood 1990, 76 (1), 1-16.

6. Broad, A. J., et al., The lethality in mice of dangerous Australian and other snake venom. Toxicon 1979, $17(6), 661-4$.

7. Masci, P. P., et al., Purification and characterization of a prothrombin activator from the venom of the Australian brown snake, Pseudonaja textilis textilis. Biochem Int 1988, 17 (5), 825-35.

8. Speijer, H., et al., Prothrombin activation by an activator from the venom of Oxyuranus scutellatus (Taipan snake). J Biol Chem 1986, 261 (28), 13258-67.

9. Walker, F. J., et al., Characterization of the prothrombin activator from the venom of Oxyuranus scutellatus scutellatus (taipan venom). Biochemistry 1980, 19 (5), 1020-3.

10. St Pierre, L., et al., Comparative analysis of prothrombin activators from the venom of Australian elapids. Mol Biol Evol 2005, 22 (9), 1853-64.

11. Rao, V. S., et al., The nonenzymatic subunit of pseutarin C, a prothrombin activator from eastern brown snake (Pseudonaja textilis) venom, shows structural similarity to mammalian coagulation factor V. Blood 2003, 102 (4), 1347-54.

12. Rao, V. S., et al., The catalytic subunit of pseutarin C, a group $C$ prothrombin activator from the venom of Pseudonaja textilis, is structurally similar to mammalian blood coagulation factor $\mathrm{Xa}$. Thrombosis and haemostasis 2004, 92 (3), 509-21.

13. Rao, V. S.; Kini, R. M., Pseutarin C, a prothrombin activator from Pseudonaja textilis venom: its structural and functional similarity to mammalian coagulation factor Xa-Va complex. Thrombosis and haemostasis 2002, 88 (4), 611-9.

14. Bos, M. H., et al., Venom factor $\vee$ from the common brown snake escapes hemostatic regulation through procoagulant adaptations. Blood 2009, 114 (3), 686-92.

15. Bos, M. H.; Camire, R. M., Procoagulant adaptation of a blood coagulation prothrombinase-like enzyme complex in australian elapid venom. Toxins (Basel) 2010, 2 (6), 1554-67.

16. Lechtenberg, B. C., et al., Crystal structure of the prothrombinase complex from the venom of Pseudonaja textilis. Blood 2013, 122 (16), 2777-83.

17. Schreuder, M., et al., Evolutionary Adaptations in Pseudonaja Textilis Venom Factor X Induce Zymogen Activity and Resistance to the Intrinsic Tenase Complex. Thrombosis and haemostasis 2020.

18. Verhoef, D., et al., Functional implications of the unique disulfide bond in venom factor $V$ from the Australian common brown snake Pseudonaja textilis. Toxin Reviews 2014, 33 (1-2), 37-41.

19. Egan, J. O., et al., The effect of Arg306-->Ala and Arg506-->GIn substitutions in the inactivation of recombinant human factor Va by activated protein C and protein S. Protein Sci 1997, 6 (9), 2016-27.

20. Dahlback, B.; Villoutreix, B. O., The anticoagulant protein C pathway. FEBS Lett 2005, 579 (15), $3310-6$.

21. Kalafatis, M., et al., The mechanism of inactivation of human factor $V$ and human factor Va by activated protein C. J Biol Chem 1994, 269 (50), 31869-80.

22. Kalafatis, M.; Mann, K. G., Role of the membrane in the inactivation of factor Va by activated protein C. $J$ Biol Chem 1993, 268 (36), 27246-57.

23. Nicolaes, G. A., et al., Peptide bond cleavages and loss of functional activity during inactivation of factor Va and factor VaR506Q by activated protein C. J Biol Chem 1995, 270 (36), 21158-66.

24. Mann, K. G., et al., Activated protein C cleavage of factor Va leads to dissociation of the A2 domain. J Biol Chem 1997, 272 (33), 20678-83.

25. Higgins, D. L.; Mann, K. G., The interaction of bovine factor $\mathrm{V}$ and factor $\mathrm{V}$-derived peptides with phospholipid vesicles. J Biol Chem 1983, 258 (10), 6503-8.

26. Toso, R.; Camire, R. M., Removal of B-domain sequences from factor $V$ rather than specific proteolysis underlies the mechanism by which cofactor function is realized. J Biol Chem 2004, 279 (20), 21643-50.

27. Krishnaswamy, S.; Walker, R. K., Contribution of the prothrombin fragment 2 domain to the function of factor $\mathrm{Va}$ in the prothrombinase complex. Biochemistry 1997, 36 (11), 3319-30.

28. Verhoef, D., et al., Engineered factor Xa variants retain procoagulant activity independent of direct factor Xa inhibitors. Nat Commun 2017, 8 (1), 528. 
29. van der Neut Kolfschoten, M., et al., Factor Va is inactivated by activated protein $C$ in the absence of cleavage sites at Arg-306, Arg-506, and Arg-679. J Biol Chem 2004, 279 (8), 6567-75.

30. Rapaport, D. C., The Art of Molecular Dynamics Simulation. J. Chem. Educ. 1999, 76 (2), 171.

31. Shim, J. Y., et al., A model for the unique role of factor Va A2 domain extension in the human ternary thrombin-generating complex. Biophysical chemistry 2015, 199, 46-50.

32. Krieger, E.; Vriend, G., YASARA View - molecular graphics for all devices - from smartphones to workstations. Bioinformatics 2014, 30 (20), 2981-2.

33. Maier, J. A., et al., ff14SB: Improving the Accuracy of Protein Side Chain and Backbone Parameters from ff99SB. J Chem Theory Comput 2015, 11 (8), 3696-713.

34. Ryckaert, J., et al., Numerical integration of the cartesian equations of motion of a system with constraints: molecular dynamics of n-alkanes. J. Chem. Theory Comput. 1977, 23 (3), 327-341.

35. Case, D. A., et al., AMBER 2016. University of California, San Francisco 2016.

36. Genheden, S.; Ryde, U., The MM/PBSA and MM/GBSA methods to estimate ligand-binding affinities. Expert Opin Drug Discov 2015, 10 (5), 449-61.

37. Norstrom, E., et al., Functional characterization of recombinant FV Hong Kong and FV Cambridge. Blood 2002, 100 (2), 524-30.

38. Rosing, J., et al., Effects of protein $\mathrm{S}$ and factor Xa on peptide bond cleavages during inactivation of factor Va and factor VaR506Q by activated protein C. J Biol Chem 1995, 270 (46), 27852-8.

39. Walker, F. J., Regulation of activated protein $\mathrm{C}$ by a new protein. A possible function for bovine protein $\mathrm{S}$. J Biol Chem 1980, 255 (12), 5521-4.

40. Minh Le, T. N., et al., Gene duplication of coagulation factor $V$ and origin of venom prothrombin activator in Pseudonaja textilis snake. Thrombosis and haemostasis 2005, 93 (3), 420-9.

41. Kalafatis, M.; Mann, K. G., Factor V: Dr. Jeckyll and Mr. Hyde. Blood 2003, 101, 20-30.

42. Lollar, P.; Parker, E. T., Structural basis for the decreased procoagulant activity of human factor VIII compared to the porcine homolog. J Biol Chem 1991, 266 (19), 12481-6.

43. Lollar, P.; Parker, C. G., pH-dependent denaturation of thrombin-activated porcine factor VIII. J Biol Chem 1990, 265 (3), 1688-92.

44. Bolton-Maggs, P. H.; Pasi, K. J., Haemophilias A and B. Lancet 2003, 361 (9371), 1801-9.

45. Graf, L., Extended Half-Life Factor VIII and Factor IX Preparations. Transfus Med Hemother 2018, 45 (2), 86-91.

46. Zollner, S., et al., Non-clinical pharmacokinetics and pharmacodynamics of rVIII-SingleChain, a novel recombinant single-chain factor VIII. Thromb Res 2014, 134 (1), 125-31.

47. Klamroth, R., et al., Comparative pharmacokinetics of rVIII-SingleChain and octocog alfa $(\operatorname{Advate}((\mathrm{R})))$ in patients with severe haemophilia A. Haemophilia 2016, 22 (5), 730-8. 


\section{Supplementary information}

\section{Methods}

Native PAGE The structural integrity of FV variants was assessed by Native-PAGE using pre-cast 3$12 \%$ Bis-Tris gels (Life technologies; Carlsbad, CA, USA). APC-proteolyzed FV variants were loaded on gels using Native Sample Buffer (Life technologies; Carlsbad, CA, USA) followed by staining with Coomassie Brilliant Blue R-250.

Thermal decay of APC-treated FVa variants Thrombin-activated ptFV variants (100 nM) were treated with APC $(150 \mathrm{nM})$ in the presence of PCPS $(50 \mu \mathrm{M})$ for 15 minutes at $37^{\circ} \mathrm{C}$ and were subsequently incubated in a water bath at $52^{\circ} \mathrm{C}$. At indicated timepoints, an aliquot was withdrawn and diluted in icewater. Cofactor activity of FVa aliquots were measured as described as detailed in 'Materials and Methods'.

Stability of APC-treated FVa variants at increased ionic strength Thrombin-activated ptFVa variants $(50 \mathrm{nM})$ were treated with APC $(75 \mathrm{nM})$ for 5 hours at $37^{\circ} \mathrm{C}$ in the presence of PCPS $(50 \mu \mathrm{M})$ and increasing concentrations of $\mathrm{NaCl}(150-2000 \mathrm{mM})$ in assay buffer. The initial velocity of thrombin generation by APC-treated FVa $(0.1 \mathrm{nM})$ was determined as described as detailed in 'Materials and Methods'.

\section{Figures}

A

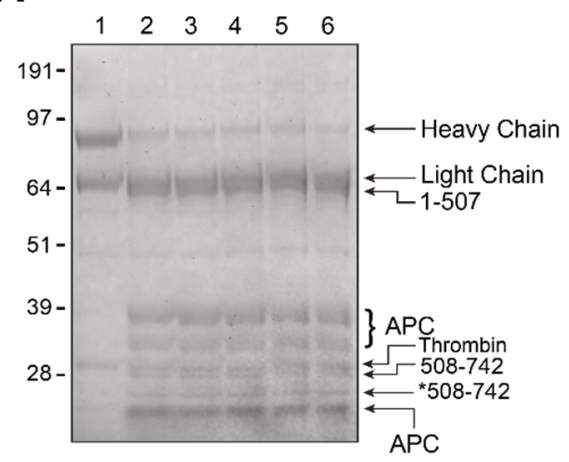

B

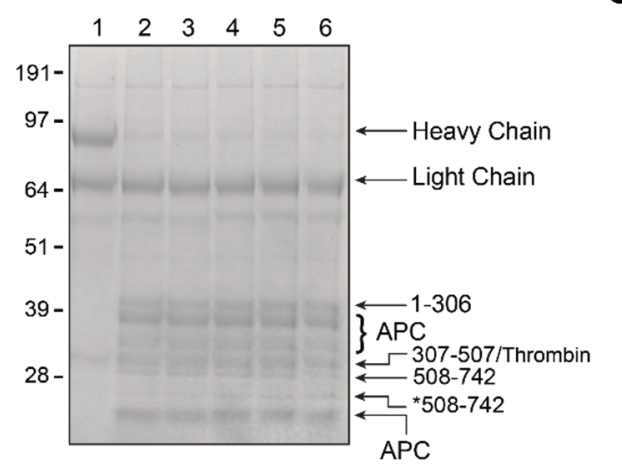

C

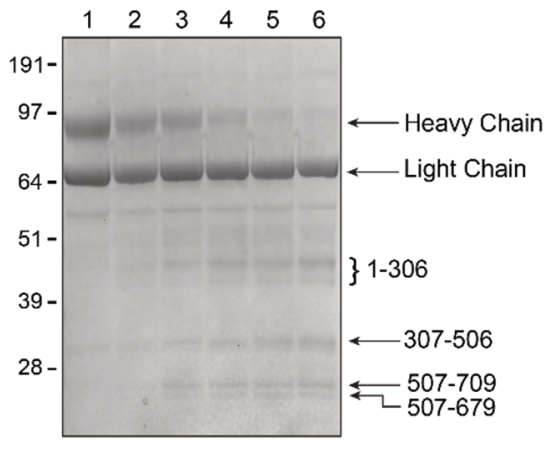

Supplementary Figure 1. Activated protein $C(A P C)$ treatment of human and $P$. textilis factor Va variants. $S D S-$ PAGE of APC-treated ptFVa (A), ptFVa-h306 S-S (B), or $h F V a(C)$ (3 $\mu$ g per lane) under reducing conditions and visualized by staining with Coomassie Brilliant Blue R-250. Lanes 1-6 represent time samples quenched at 0, 0.5, 1, 2.5, 5, and $15 \mathrm{~min}$. Relevant fragments including the heavy chain (A1-A2) and light chain (A3-C1-C2) and the apparent molecular weights of the standards are indicated. We hypothesize that *508-742 is cleaved at a C-terminal position because its $N$-terminal sequence was determined to be the same as 508-742 (see Supplementary Figure 2). The data are representative of two to three independent experiments. 


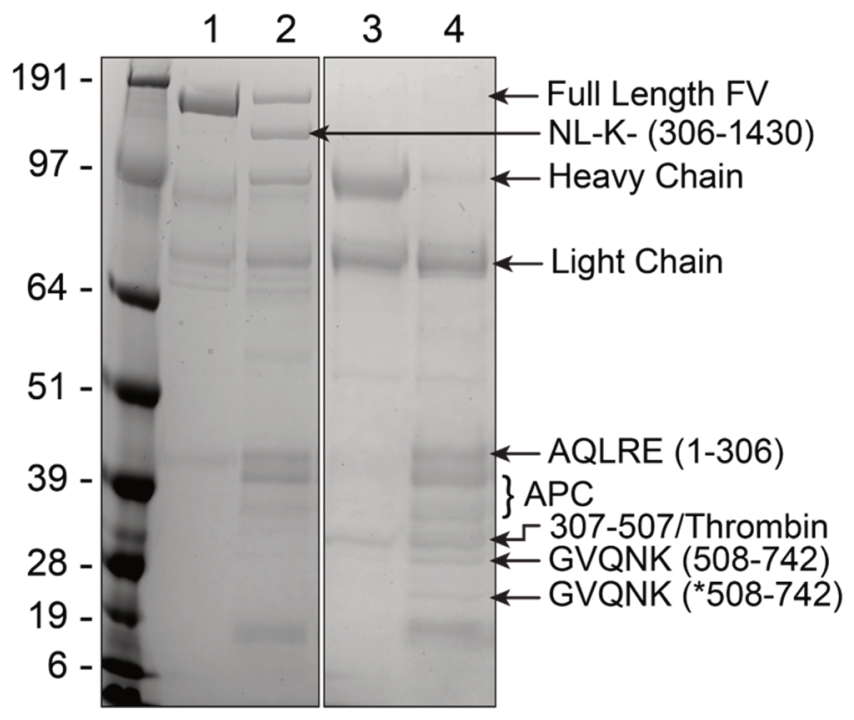

Supplementary Figure 2. Characterization of APC cleavage sites in ptFV-h306. SDS-PAGE of APC-treated ptFVh306 and ptFVa-h306 (3 $\mu$ g per lane) under reducing conditions and visualized by staining with Coomassie Brilliant Blue R-250. Lane 1, ptFV-h306 at $t=0$; lane 2, ptFV-h306 at $t=0.5 \mathrm{~min}$; lane 3, ptFVa-h306 at $t=0$; lane 4, ptFVa$h 306$ at $t=0.5 \mathrm{~min}$. $\mathrm{N}$-terminal sequence results of the indicated protein bands and the apparent molecular weights of the standards are shown.

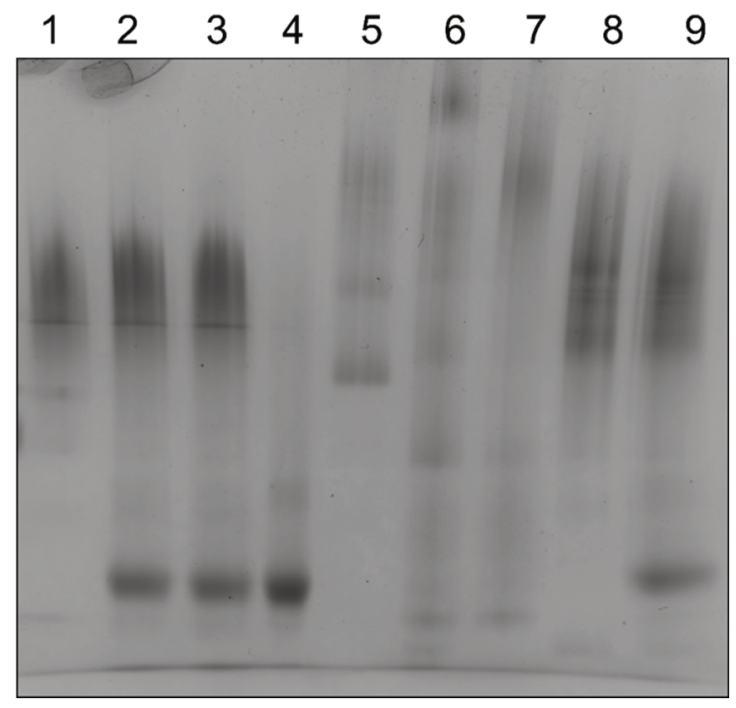

Supplementary Figure 3. Native-PAGE analysis of APC-proteolyzed human and $P$. textilis factor $V$ variants. Lane 1, ptFV at $t=0$; lane 2, APC-treated ptFV at $t=15$; lane 3, APC-treated ptFV at $t=60$; lane 4, APC; lane 5, $h F V$ at $t=0$; lane 6, APC-treated $h F V$ at $t=15$; lane 7, APC-treated $h F V$ at $t=60$; lane 8, ptFV-h306 S-S at $t=0$; lane 9, APCtreated ptFV-h306 S-S at $t=60$. The data are representative of two independent experiments. 


\section{A}

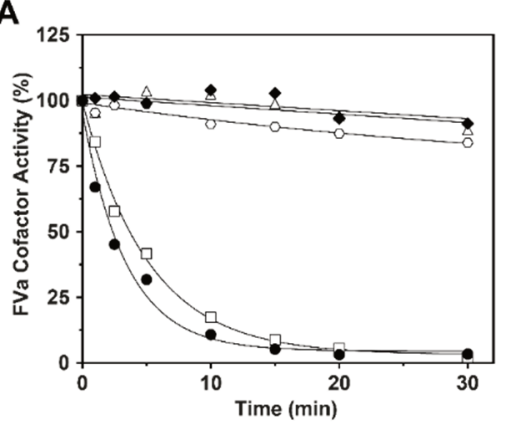

B

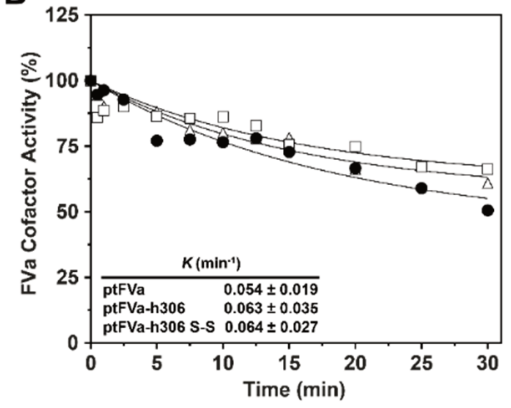

C

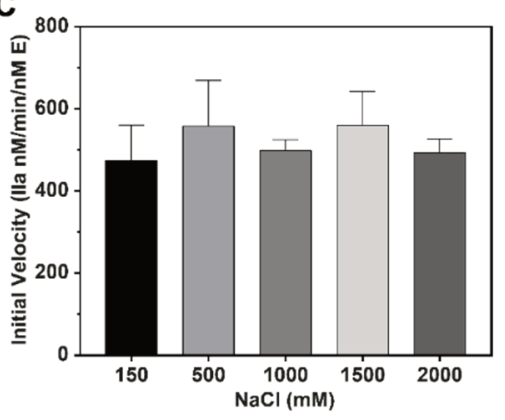

Supplementary Figure 4. Factor Va cofactor activity in conditions of increased temperature or high ionic strength. (A) Reaction mixtures containing $20 \mathrm{nM}$ hFVa (circles), hFVa-pt306 (squares), ptFVa (diamonds), ptFVa-h306 (triangles), or ptFVa-h306 S-S (hexagon) were incubated at $52^{\circ} \mathrm{C}$. At selected time intervals, samples were removed and diluted on ice, upon which they were assayed for cofactor activity employing a FV-specific PT-based clotting assay. (B) APC-treated ptFVa (circles), ptFVa-h306 (squares), or ptFVa-h306-SS (triangles) were incubated at $52^{\circ} \mathrm{C}$. At selected time intervals, samples were removed and diluted on ice, upon which they were assayed for cofactor activity by determining the initial velocity of prothrombin conversion as described in 'Materials and Methods'. Cofactor decay rate constants \pm 1 S.D. (insets) were determined by fitting the data to a one-phase decay function by non-linear regression. The data are representative of two to three independent experiments. (C) ptFVa-h306 S$S(50 \mathrm{nM})$ was treated with $\mathrm{APC}(75 \mathrm{nM})$ at $37^{\circ} \mathrm{C}$ in the presence of $50 \mu \mathrm{M}$ PCPS and increasing concentrations of $\mathrm{NaCl}(150-2000 \mathrm{mM})$ in assay buffer. After 5 hours of incubation, samples were removed and diluted in assay buffer for cofactor activity assessment by determining the initial velocity of prothrombin conversion. Data represent the mean \pm 1 S.D. and are representative of two independent experiments. 
A

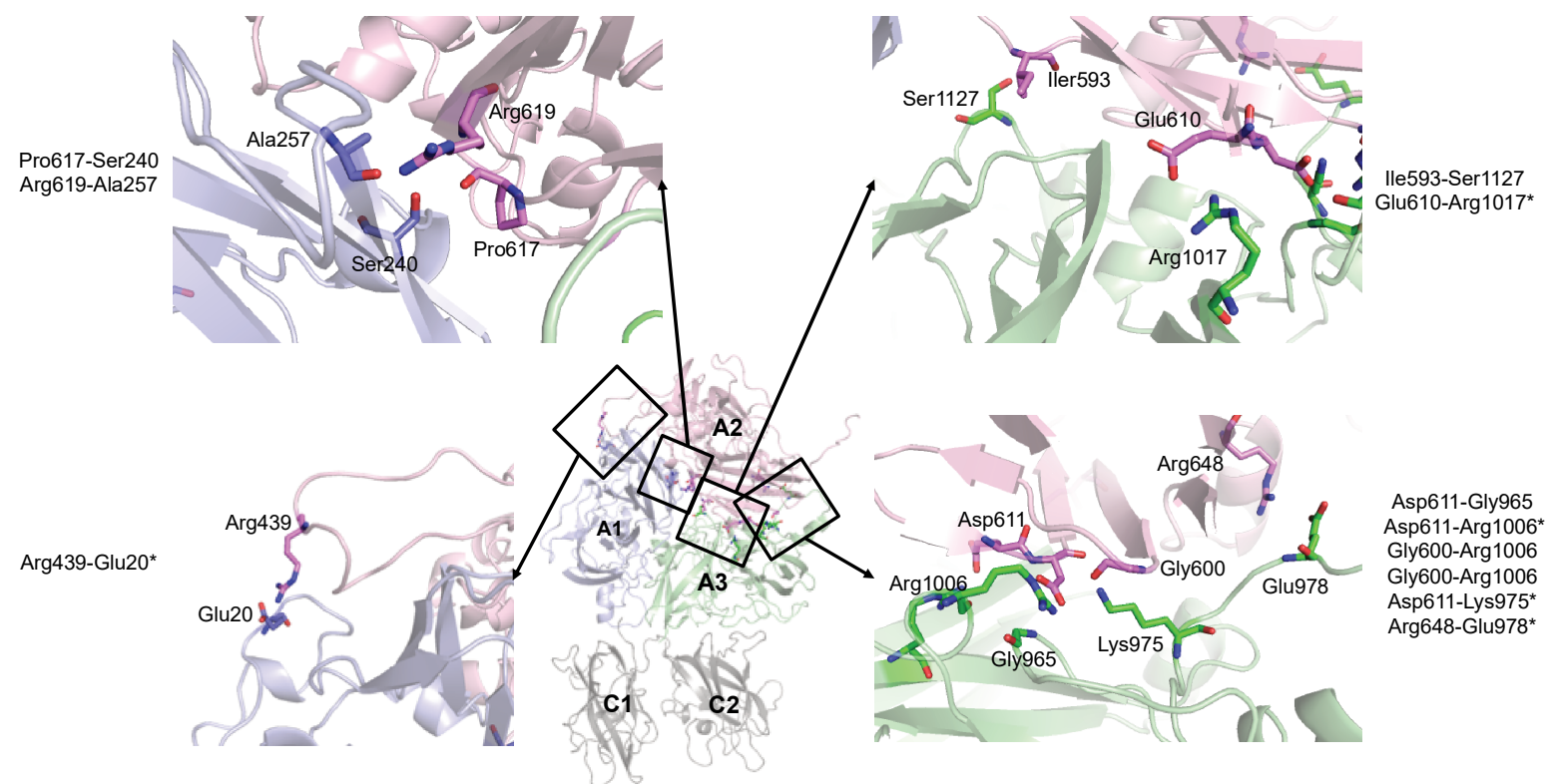

B

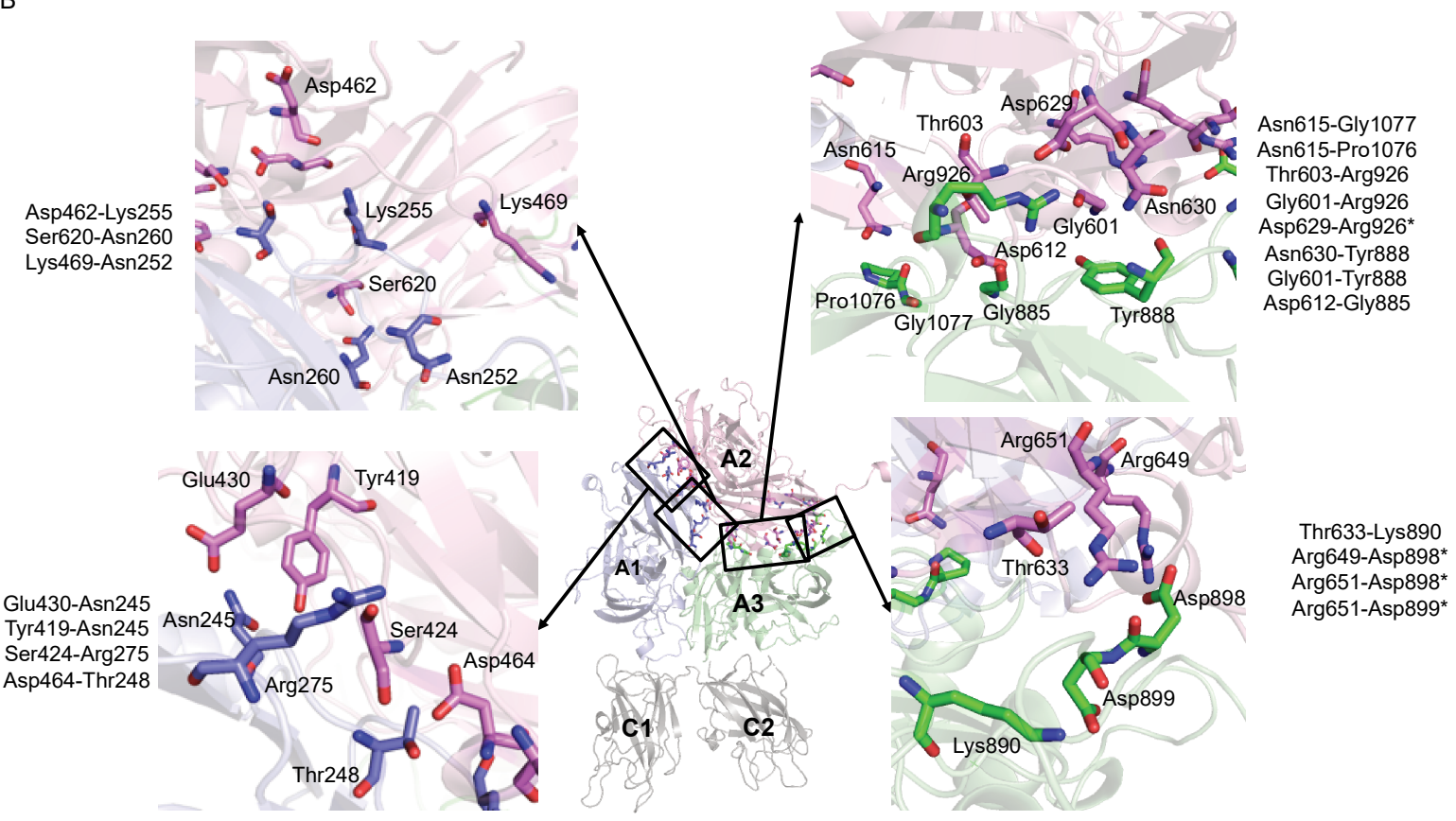

Supplementary Figure 5. Structural representation of residues involved in key A2-A1 or A2-A3 domain hydrogen bond interaction pairs (A-B). Residues involved in key hydrogen bond interactions, as defined in 'Materials and Methods', between the $A 2$ domain (pink) and the $A 1$ (blue) or A3 domain (green) in hFVa (A) or ptFV (B) are shown in stick configuration. The inserts display zoomed-in regions and depict the interaction pairs. The A1, A2, A3, C1, and C2 domains are indicated. * Denotes charged interactions. 


\section{Sequence alignment}

\begin{tabular}{|c|c|c|}
\hline \multirow{4}{*}{$\begin{array}{l}\text { Human } \\
\text { P.textilis }\end{array}$} & \multicolumn{2}{|l|}{ Loop1 } \\
\hline & \multirow{2}{*}{\multicolumn{2}{|c|}{$\begin{array}{l}\text { AQLRQFYVAAQGISWSYRPEPTNS-SLNLSVTSFKKIVYREYEPYFKKEKPQSTISGLLG } \\
\text { AQLREYHIAAQLEDWDYNPQPEELSRLSESDLTFKKIVYREYELDFKQEKPRDALSGLLG }\end{array}$}} \\
\hline & & \\
\hline & \multicolumn{2}{|l|}{ 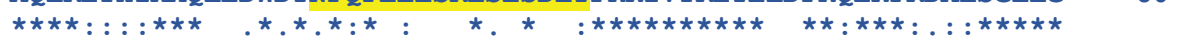 } \\
\hline Human & PTLYAEVGDI IKVHFKNKADKPLSIHPQGIRYSKLSEGASYLDHTFPAEKMDDAVAPGRE & \\
\hline P. textilis & \multicolumn{2}{|l|}{ PTLRGEVGDSLI IYFKNFATQPVSIHPQ̋SAVYNKWSEGSSYSDGTSDVERLDDAVPPGQS } \\
\hline & \multicolumn{2}{|l|}{ 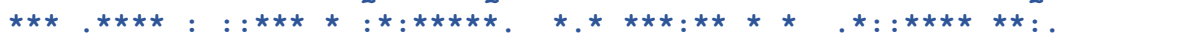 } \\
\hline Human & YTYEWSISEDSGPTHDDPPCLTHIYYSHENLIEDFNSGLIGPLLICKKGTLTEGGTQKTF & \\
\hline P.textilis & \multicolumn{2}{|l|}{ FKYVWNITAE IGPKKADPPCLTYAYYSHVNMVRD FNSGLIGALLICKEGSLNANGSQKFF } \\
\hline & \multicolumn{2}{|l|}{ 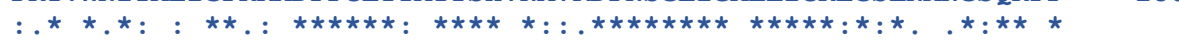 } \\
\hline Human & DKQIVLLFAVFDESKSWSQSSSLMYTVNGYVNGTMPDITVCAHDHISWHLLGMSSGPELF & \\
\hline P. textilis & NREYVLMFSVFDESKNWYRKPSLQYTINGFANGTLPDVQACAYDHISWHLIGMSSSPEIF & \\
\hline & 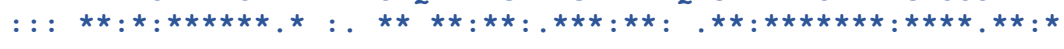 & \\
\hline Human & SIHFNGQVLEQNHHKVSAITLVSATSTTANMTVGPEGKWIISSLTPKHLQAGMQAYIDIK & \\
\hline P. textilis & SVHFNGQTLEQNHYKVSTINLVGGASVTADMSVSRTGKWLISSLVAKHLQAGMYGYLNIK & \\
\hline & \multicolumn{2}{|l|}{ 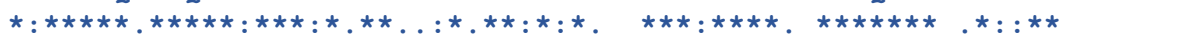 } \\
\hline Human & NCPKKTRNLKKITREQRRHMKRWEYFIAAEEVIWDYAPVI PANMDKKYRSQHLDNFSNQI & \\
\hline P. textilis & DCGNPDTLTRKLSFRELMKIKNWEYFIAAEEITWDYAPEIPSSVDRRYKAQYLDNFSNFI & \\
\hline & 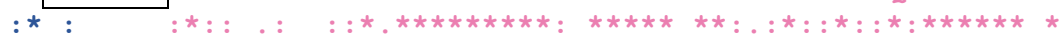 & \\
\hline Human & GKHYKKVMYTQYEDESFTKHTVNPNMKEDGILGPI IRAQVRDTLKIVFKNMASRPYSIYP & \\
\hline \multirow[t]{3}{*}{ P.textilis } & \\
\hline & \multicolumn{2}{|l|}{ 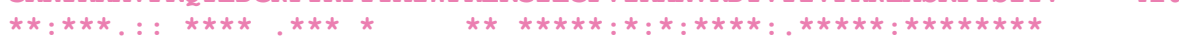 } \\
\hline & 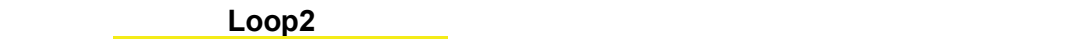 & \\
\hline Human & HGVTFSPYEDEVNSSFTSGRNNTMIRAVQPGETYTYKWNILEFDEPTENDAQCLTRPYYS & \\
\hline P. textilis & HGVSVSKDAEGAIYPSDPKENITHGKAVEPGQVYTYKWTVLDTDEPTVKDSECITKLYHS & \\
\hline & \multicolumn{2}{|l|}{ 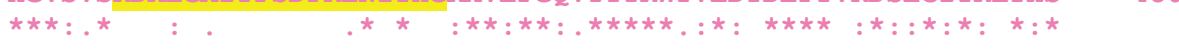 } \\
\hline Human & DVDIMRDIASGLIGLLLICKSRSLDRRGIQRAADIEQQAVFAVFDENKSWYLEDNINKFC & \\
\hline P. textilis & AVDMTRDIASGLIGPLLVCKHKALSVKGVQNKADVEQHAVFAVFDENKSWYLEDNIKKYC & \\
\hline & 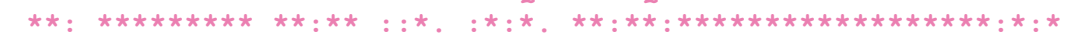 & \\
\hline Human & ENPDEVKRDD PKFYESNIMSTINGYVPESITTLGFCFDDTVQWHFCSVGTQNEILTIHFT & \\
\hline P. textilis & SNPSAVKKDDPKFYKSNVMYTLNGYASDRTEVLRFHQSEVVQWHLTSVGTVDEIVPVHLS & \\
\hline & 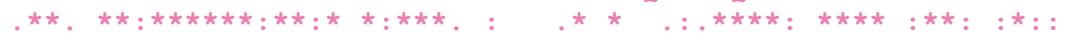 & \\
\hline Human & GHSFIYGKRHEDTLTLFPMRGESVTVTMDNVGTWMLTSMNSSPRSKKLRLKERDVKCIPD & \\
\hline P.textilis & GHTFLSKGKHQDILNLFPMSGESATVTMDNLGTWLLSSWGSCEMSNGMRLRFLDANYDDE & \\
\hline & 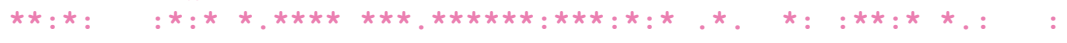 & \\
\hline Hum & DDEDS & \\
\hline P. textilis & DEGNE & \\
\hline
\end{tabular}

59

60

119

120

179

180

239

240

299

300

359

360

419

20

479

80

539

540

599

600

659

660

Supplementary Figure 6. Sequence alignment of the human and P. textilis A1-A2 domains in FVa. “*” represents identical amino acids, ":" represents very similar amino acids, "." represents somewhat similar amino acids. The A1, A2, and A3 domains are indicated in blue, pink, and green, respectively. The Arg306 region is indicated by the black frame., black arrows indicate APC cleavage sites, and loop1 and loop2 are highlighted in yellow. 
Supplementary Table 1. Apparent binding affinity for the cofactor. The apparent binding affinity of $h F X a$ or ptFXa for their respective cofactor species was obtained as described in 'Materials and Methods'. The mean values \pm S.D. are representative of two to three independent experiments.

\begin{tabular}{|l|c|c|c|c|c|}
\hline FV variants & hFV & hFV-pt306 & ptFV & ptFV-h306 & ptFV-h306 S-S \\
\hline $\boldsymbol{K}_{\mathrm{d}, \text { app }}(\mathrm{nM})$ & $0.71 \pm 0.20$ & $0.97 \pm 0.20$ & $0.83 \pm 0.18$ & $0.48 \pm 0.16$ & $0.97 \pm 0.29$ \\
\hline
\end{tabular}

Supplementary Table 2. Key hydrogen bond interaction pairs between the A2 domain and the A1 or A3 domain. Hydrogen bond pairs were identified as described in 'Materials and Methods'.

\begin{tabular}{|c|c|c|c|}
\hline Binding surfaces & hFVa & ptFVa-h306 S-S & hFVa-ptloop2 \\
\hline A2-A1 & $\begin{array}{l}\text { Arg439-Glu20* } \\
\text { Pro617-Ser240 } \\
\text { Arg619-Ala257 }\end{array}$ & $\begin{array}{l}\text { Lys469-Asn252 } \\
\text { Glu430-Asn245 } \\
\text { Tyr419-Asn245 } \\
\text { Asp464-Thr248 } \\
\text { Asp462-Lys255 } \\
\text { Ser620-Asn260 } \\
\text { Ser424-Arg275 }\end{array}$ & $\begin{array}{l}\text { Arg475-Lys254 } \\
\text { Gly421-Gly245 } \\
\text { Tyr418-Asn244 } \\
\text { Asp469-Lys254* } \\
\text { Asp480-Asn244 } \\
\text { Tyr433-Trp278 } \\
\text { Phe462-Lys254 } \\
\text { Asp480-Ser281 } \\
\text { Pro617-Ser240 } \\
\text { Asn591-Lys286 } \\
\text { Glu621-His253 } \\
\text { Arg619-Thr259 }\end{array}$ \\
\hline A2-A3 & $\begin{array}{l}\text { Ile593-Ser1127 } \\
\text { Asp611-Gly965 } \\
\text { Arg648-Glu978* } \\
\text { Asp611-Arg1006* } \\
\text { Glu610-Arg1017* } \\
\text { Gly600-Arg1006 } \\
\text { Asp611-Lys975* }\end{array}$ & $\begin{array}{l}\text { Gly601-Arg926 } \\
\text { Asn630-Tyr888 } \\
\text { Asp629-Arg926* } \\
\text { Asp612-Gly885 } \\
\text { Thr603-Arg926 } \\
\text { Asn615-Gly1077 } \\
\text { Asn615-Pro1076 } \\
\text { Arg649-Asp898* } \\
\text { Arg651-Asp898* } \\
\text { Gly601-Tyr888 } \\
\text { Thr633-Lys890 } \\
\text { Arg651-Asp899* }\end{array}$ & $\begin{array}{l}\text { Ile593-Ser1127 } \\
\text { Asp611-Arg1006* } \\
\text { Asp611-Gly965 } \\
\text { Glu610-Arg1017* } \\
\text { Asn467-Asn1145 } \\
\text { Arg608-Glu1143* } \\
\text { His609-Glu1010 } \\
\text { Arg648-Glu978* }\end{array}$ \\
\hline
\end{tabular}

${ }^{*}$ Denotes the charged interaction 


\section{Chapter}

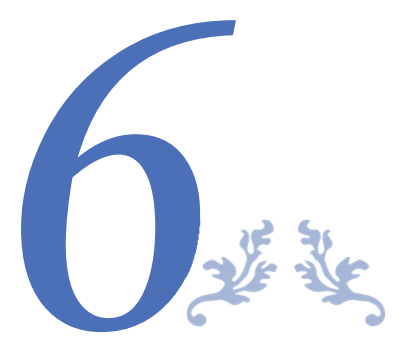

\section{Rational design of peptide agonists activating $\mathrm{C}-\mathrm{X}-\mathrm{C}$ chemokine receptor type 4 (CXCR4)}

Xiaosong Liu ${ }^{1 *}$, Alejandra Aroca-Crevillen ${ }^{2 *}$, Chris P. M. Reutelingsperger ${ }^{1}$, Andres Hidalgo ${ }^{2}$, Gerry A. F. Nicolaes ${ }^{1,{ }^{*}}$, Kanin Wichapong ${ }^{1,{ }^{*},}$

${ }^{1}$ Department of Biochemistry, Cardiovascular Research Institute Maastricht (CARIM), Maastricht University, Maastricht, The Netherlands

2Department of Cell and Developmental Biology, Centro Nacional de Investigaciones Cardiovasculares, Carlos III, Madrid, Spain

${ }^{*}$ These authors contributed equally

The contents of this chapter are under embargo 


\section{Abstract}

Background The neutrophil ageing process is mediated by CXCR2 and its ligand CXCL2 and also antagonised by CXCR4 with its ligand CXCL12. Aged neutrophils maintain neutrophil homeostasis in blood as well as enhance immune defence in a diurnal way. However, aged neutrophils can damage vessel walls in ischemia/reperfusion model. Therefore, it may represent a novel therapeutic approach for high risk of patients with vascular diseases through activating CXCR4 and limiting circadian ageing of neutrophils in blood. In this work, we aim to develop novel CXCR4 agonists which can be used to manipulate time coordinated neutrophil ageing through activating CXCR4.

Method We have applied computational methods to rationally in silico design various peptides. The potential candidates, as determined by binding free energy values, were selected for further synthesis and consequently tested in vitro to evaluate their biological activities in terms of activation of cellular pathways mediated through CXCR4.

Results In total, we have in silico designed over 70 peptides and were able to select top-three candidates for synthesis and experimental testing. All three peptides trigger cellular activation via the ERK1/2 mitogen-activated protein kinase pathway.

Conclusion Collectively, we have successfully developed novel CXCR4 agonists, and by utilisation of computational methods, as presented here, we can significantly accelerate the peptide design process and reduce the number of synthesised and tested peptides. Therefore, the computational protocols as demonstrated in this work can also be applied to design and develop either agonists or antagonists to target other GPCR receptors.

Keywords: C-X-C chemokine receptor type 4 (CXCR4), CXCL12, CXCR4 agonist, Computer-Aided Molecular Design (CAMD), Peptide Design 
Chapter

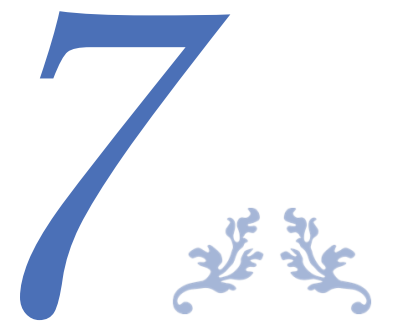

General discussion 


\subsection{Introduction}

Cardiovascular disease (CVD) encompasses a class of disorders affecting the heart and/or blood vessels, such as thrombosis, atherosclerosis and myocardial ischemia1. According to the World Health Organization, CVD leads to an approximate 17.9 million deaths each year worldwide (about $31 \%$ of all deaths), making CVD the number one cause of death globally. The development of therapeutics for the treatment of CVD is challenging, as traditional approaches associated with experimental work are expensive and time-consuming. However, during the last decades, structural bioinformatics has emerged as an efficient and powerful science that facilitates the development of novel disease treatments. Structural bioinformatics is a science field that can be used to study the structure, movement and interaction of three-dimensional (3D) biological molecules ${ }^{2}$ and discover/design lead compounds towards a specific target in CVD.

In this thesis, we discuss advantages and challenges of structural bioinformatics tools in improving understanding of mechanisms at the structural level and we present examples in which protein targets in CVD are structurally inspected by these tools to discover modulatory molecules that may evolve into drugs. Chapter 2 introduces in detail the modulator design workflow using structural bioinformatics tools based on protein-protein interactions and Chapter 3 reviews peptide arginine deiminase 4 (PAD4), a new and interesting target in CVD in regard to its structure-function relationships. Chapters $\mathbf{4}$ and $\mathbf{5}$ demonstrate how structural bioinformatics methods can help us gain better understanding of mechanisms associated with CVD by studying the dynamics of target proteins and their interactions at the atomic level. Chapter 6 describes how structural bioinformatics forwards drug discovery in an efficient and cost-effective way through rational design of peptidic modulators targeting pathological protein-protein interactions involved in CVD.

\subsection{Computational methods assist experimental methods in the study of protein structures}

In silico analysis of protein structure and dynamics through available structural bioinformatics tools (such as homology modeling, molecular dynamic (MD) simulations, and binding free energy (BFE) calculations) assist researchers in revealing structural features and characteristics of the protein of interest. Computational approaches when combined with experimental approaches allow us to obtain insight into protein structure with an unparalleled view and uncover hidden features that determine protein function. In this thesis, we studied the protein structures of two CVD targets by performing a combination of computational and experimental methods.

\section{PAD4 as the target of interest}

PAD4 belongs to the peptide arginine deiminases (PADs) family which catalyzes the citrullination process by converting arginine into citrulline. Amongst all PADs members, PAD4 gains increasing attention as it is expressed inside the cell nucleus and drives neutrophil extracellular trap (NET) formation by citrullination of nuclear histones. In chapter 3 we provide an overview of the current knowledge on PAD4, explicitly focusing on its structural and functional basis, including the regulation of 
its activity, its role in progression of diseases and its potential inhibitors. PAD4 requires calcium to become activated; calcium binding induces the PAD4 structure from its inactive form (calcium-free form) to its active form (calcium-bound form) ${ }^{3}$. PAD4 is implicated in thrombosis and sepsis mainly through induction of NETs. However, there are no specific and potent PAD4 inhibitors available. The class of discovered covalent inhibitors such as $\mathrm{Cl}$-amidine and F-amidine, show unspecific inhibition to almost all PADs members as it forms covalent bonds with the cysteine residue at the active site (formed by D350, H471, D473, C645) which is conserved in all PADs members, except PAD6. Although Thompson and colleagues put great effort into the optimization of these covalent inhibitors, it remains a challenge to improve their binding affinity and specificity towards PAD44. Up to now, the most promising PAD4 inhibitor is derived from the class of GSK reversible inhibitors, such as GSK199 and GSK484, which show nanomolar levels of binding affinity. Nevertheless, they bind the inactive form of PAD4, from which the exact function in physiological cellular pathways is unclear ${ }^{5}$. Therefore, the consequences of inhibiting such inactive form of PAD4 in vivo remain unknown. By analysis of the active site in PAD4 and other isoforms within the PAD family, we revealed that some residues at the entrance of the substrate binding pocket in PAD4 are not conserved in all PAD members. For instance, R374 exists in both PAD1 and PAD4 but not in PAD2 and PAD3, whilst R639 and H640 only exist in PAD4. Therefore, it is essential to include these specific residues in future research screening and selecting PAD4-specific inhibitors. Additionally, it was characterized that the "front door" in PAD4 functions as the substrate binding pocket, and the "back door" functions as the solvent channel for the release of the product ammonia after citrullination ${ }^{6}$. Although the "front door" represents the major inhibitor binding site in PAD4, it is still an attractive approach to target the "back door" in the discovery of novel and specific PAD4 inhibitors ${ }^{7}$.

In the last decades, PAD4 has received increasing attention based on its role in the pathological of diseases and the discovery of its novel inhibitors. Nevertheless, there is still a lack of knowledge about the regulation of PAD4 under physiological conditions. It was reported by Andrade, $\mathrm{F}$ et al. ${ }^{8}$ that PAD4 can citrullinate itself, referred to as autocitrullination. It was hypothesized that PAD4 may regulate its activity through such self-controlling mechanism. It remains controversial whether autocitrullination indeed influences PAD4 activity. In chapter 4 , we performed both experimental and computational methods to study the effect of autocitrullination on PAD4 activity. The experimental approaches found that the autocitrullination of PAD4 occurs in a time- and concentration-dependent manner. After incubating PAD4 alone for 2 hours in the presence of calcium, mass spectrometry analysis (MS) has identified 23/27 citrullinated arginine residues in PAD4 (85\% of total). Given the longer incubation time of PAD4 and the improved sensitivity of MS analysis, this percentage may even increase further. It was reported that the properties of neighboring residues around the target arginine alters the preference of PAD4 for substrate 9 . However, we did not find either a relationship between the position of arginine in sequence and the nature of its neighboring residues or a connection of citrullinated arginines and their solvent accessibility. Based on these experiments, we used the aliquots of PAD4 at $2 \mathrm{~h}$ incubation time as the citrullinated PAD4 (citPAD4) to compare the kinetics of PAD4 and citPAD4. By applying different sized substrates, our results show that citPAD4 and PAD4 display comparable activities towards 
substrates, Our conclusions are consistent with a previous study by Slack and coworkers ${ }^{10}$ but are in contrast obtained results by Andrade and coworkers ${ }^{8}$. In Andrade's study, they used the cell lysate as the substrate to evaluate the activities of PAD4 and citPAD4. Considering the numerous proteins and the complex environment in cell lysate, it is possible that a protein prefers to bind citrullinated PAD4 and blocks its catalytic activity.

In order to obtain insight into the structural changes of citPAD4 at the atomic level, computational approaches were applied, including homology modelling, molecular dynamic simulations and binding free energy calculations. The homology modeling module in YASARA ${ }^{11}$ was used to create a full length PAD4 structural model with a predicted nuclear localization signal (NLS) region and missing loops which are flexible at the $\mathrm{N}$-terminal domain. This full length PAD4 model that was built by homology modelling was used as the starting structure for PAD4 in MD simulations. The PAD4 model structure carrying 23 citrulline residues corresponding to the mass spectrometry results was taken as the starting structure for citPAD4 in MD simulations. After performing $100 \mathrm{~ns}$ of MD simulations on the structure of both PAD4 and citPAD4 and their structures with a same substrate, we were able to monitor the protein structure stability and substrate binding stability. The root-mean-square deviation (RMSD) values based on the substrate binding pocket converged, which indicates a stable binding pocket is preserved in citPAD4. The conformational change mainly resides in the $\mathrm{N}$-terminal subdomain I. PAD4 adopts two immunoglobulin-like subdomains at the $\mathrm{N}$-terminal domain which are likely to form protein-protein interactions involved in cell signaling ${ }^{3,12}$. Our findings may explain the previously obtained results that autocitrullination alters the interactions between PAD4 and its partners ${ }^{10}$. Furthermore, the calculated binding free energies also show that the substrate has no preference for binding to PAD4 or citPAD4, which is consistent with experimentally obtained results by western blot. The decomposition analysis shows key residues on PAD4 for binding to its substate are W347, D473 and D350. From hydrogen bond analysis, R374 and R639 interact with the substrate through backbones-to-backbones interactions which were not altered by citrullination, R372 does not directly form hydrogen bonds with the targeted arginine within the substrate, but with neighboring residues. Although R372, R374 and R639 which locate at the entrance of catalytic pocket and stabilize the substrate were also citrullinated, our in silico studies indicate that citrullination of them only have little to no effect on PAD binding to its substrate. The combined experimental and computational methods demonstrated that autocitrullination does not play a role in the regulation of PAD4 activity. Given that a large number of charges are removed from the PAD4 surface, citrullinated PAD4 may bind to preferred protein partners and allow the blockade of substrate binding site in vivo, thereby indirectly influencing PAD4 activity. Future works still need to investigate the effect of autocitrullination on PAD4 in its physiological environment.

\section{FV as the target of interest}

Coagulation factor $\mathrm{V}(\mathrm{FV})$ plays an essential role in blood coagulation. When $\mathrm{FV}$ is activated into $\mathrm{FVa}$, it works as the cofactor for factor $\mathrm{Xa}$ by the formation of the prothrombinase complex ${ }^{13}$. This complex converts prothrombin into thrombin, a key enzyme in coagulation that leads to the formation of blood clots. To help downregulate this procoagulant response, human $\mathrm{FVa}(\mathrm{hFVa})$ is cleaved and inactivated 
by activated protein $\mathrm{C}(\mathrm{APC})^{14}$. However, it was found that the Australian snake Pseudonaja textilis contains a venom-derived factor $\mathrm{Va}(\mathrm{ptFVa})$ that retains its cofactor function despite APC-dependent proteolysis ${ }^{15}$. As we know that the proteins 3D-structure determines its function, it is interesting to uncover the structural features that make these two FVa homologues exert opposite effects in the blood coagulation system.

Chapter 5 describes the combination of experimental and computational approaches to reveal the mechanistic principles responsible for the functional resistance of ptFVa to APC. In hFVa, APC cleavage at Arg306 and Arg506 results in the release of the A2 domain from the A1-A2-A3 domain trimer, and consequent loss of cofactor activity. Although ptFVa is cleaved by APC at a position that is homologous to human Arg506 (Lys507), another cleavage site (human Arg306-like) is absent. Furthermore, ptFV additionally carries a unique disulfide bond that covalently links the A2 and A3 domains. In vitro molecular biology experiments generated a chimeric ptFV variant (ptFVa-h306 S-S) that contains the human Arg306 cleavage site and lacks the unique A2-A3 domain disulfide bond. However, assessment of FVa cofactor function showed that APC-cleaved ptFVa-h306 S-S still has full procoagulant activity despite cleavage at Arg306 and disruption of the unique disulfide bond. Size-exclusion chromatography demonstrated that A2-domain dissociation was prevented in both ptFVa and ptFVa-h306 S-S. In conclusion, the experimental data demonstrated that none of these structural elements play a role in the functional resistance of ptFVa to APC.

To discover which structural features prevent $\mathrm{A} 2$ domain dissociation and convert ptFV into an apparent APC-resistant procoagulant protein, we continued employing computational methods in this study. To gain more insights into the A2 domain binding with the rest domains at molecular levels after APC cleavage, we simulated both hFVa and ptFVa-h306 S-S (ptFVa holding a human Arg306 cleavage site and disrupted disulfide bond) for $100 \mathrm{~ns}$. The BFE was then calculated for the interaction between the $\mathrm{A} 2$ domain and $\mathrm{A} 1+\mathrm{A} 3$ domains at the beginning (first $20 \mathrm{~ns}$ ) and the end (last 20ns) of MD simulations for both hFVa and ptFVa-h306 S-S. The calculated BFEs showed that the A2 domain lost its binding to the A1+A3 domains in hFVa while this domain slightly gained in binding strength in ptFVa-h306 S-S along MD simulation time. In hFVa, the major loss of binding between $\mathrm{A} 2$ and the A1-A3 dimer arose from the interaction between the $A 2$ and $A 1$ domain, which may trigger the dissociation of the A2 domain. Furthermore, the hydrogen bond analysis displayed that the number of hydrogen bonds between the A2 and A1 domain decreases significantly upon APC-mediated proteolysis in hFVa.

More importantly, our in silico results found that the loss of binding between the A1 and A2 domain may attribute to a loop (called loop2) in the A2 domain of hFVa. Although sequence alignments show that hFVa and ptFVa share high sequence similarity $(71 \%)$, the loop2 displays a major conformational change in hFVa and ptFVa. In hFVa, the loop2 appears to be uncompact with big solvent accessible surface areas but gets stabilized by interacting with a loop in the A1 domain (called loop1) before APC cleavage. However, interactions between loop2 and loop1 became unstable after APC cleavage in hFVa, which is probably the concealed mechanism that triggers the dissociation of the A2 domain. In ptFVa, 
on the other hand, loop2 has a compact conformation and does not interact with loop1 in the A1 domain. To prove the role of loop2 in the A2 domain dissociation in $\mathrm{hFVa}$, we in silico generated a chimeric $\mathrm{hFVa}$ (hFVa-ptloop2) by switching the ptFVa loop2 into hFVa. Using MD simulations our results showed that hFVa-ptloop2 has a stabilized structure and a significantly improved binding between the A2 and A1-A3 domains. Therefore, we highlighted a key feature that may play a central role in preventing A2 domain dissociation in ptFV by the application of a combined experimental and computational method.

Our findings could provide a strategy for generating novel and stable FV or factor VIII (FVIII) variants as therapeutics for the treatment of bleeding disorders such as hemophilia. Activated FVIII (FVIIIa) remains a structure stability issue due to a spontaneous dissociation of A2 domain, which significantly decrease its coagulant activity ${ }^{16}$. Designing of new FVIII variants has been investigated by researchers recently, for instance, the newly produced single-chain FVIII for aiming to treat haemophilia A patient showed remarkably improved binding affinity to von Willebrand factor (VWF ${ }^{17}, 18$. Our findings uncover the mechanism of A2 domain association in ptFVa, which could provide an alternative strategy for generation of new FVIII variants regarding to a structural homology between FV and FVIII. Although these findings still need to be confirmed by further experimental methods, the computational methods guide us to explore the structure and function relationships of a protein in an efficient way. This chapter supports the combination of computational and experimental methods as a powerful tool in studying protein structure.

\subsection{Rational design of peptidic modulators targeting protein-protein interactions (PPIs)}

Protein-protein interactions (PPIs) are receiving increasing interest in the drug discovery field. We have successfully applied the rational and structure-based design of peptidic modulators to many different classes of PPIs to discover and design novel modulators ${ }^{19-25}$. Chapter 2 provides a detailed workflow of the design and development of modulators (inhibitors or activators) based on PPIs, and summarizes for every step the state-of-the-art methods that have been used. In brief, this protocol starts with the identification of PPIs since it contains information on detailed atomistic interactions. The 3D structure of protein-protein complexes derived from experimental methods (such as X-ray crystallography, NMR, and cryo-EM) are commonly used in this step. However, a protein-protein complex may not always crystalize due to experimental limitations. Computational methods can overcome such issues. For instance, protein structure prediction methods can assist researchers in predicting the 3D structure of a protein-protein complex. Molecular docking can be employed to predict a likely binding mode between two proteins when individual 3D structures are available but not that of a complex structure; and the combination of MD simulations and BFE calculation can help to distinguish native-like docking poses from false positives. Designed peptides could mimic the binding epitope in PPIs, making it bind at the interfaces of the protein-protein complex. Thereafter, the exact binding mode of a peptide onto a protein can be predicted using molecular docking. The binding affinity of the peptide to protein is further estimated though BFE calculation methods. For the purpose of screening a list of designed peptides to test their biological activities, the molecular mechanics/Poisson-Boltzmann or generalized Born surface area $\mathrm{MM} / \mathrm{PB}(\mathrm{GB}) \mathrm{SA}$ method is widely employed to predict relative binding potencies as it can 130 
significantly reduce the calculation time while maintaining acceptable accuracy. Other issues to consider in peptide design involve for instance those of ease of synthesis or stability issues. Peptidic modulators have disadvantages in terms of a poor stability and short lifespan in the human body. To improve the stability and binding affinity of designed peptides, many strategies for peptide modification, such as introduction of unusual or D-amino acids or covalent bonds, capping at N/C terminus, or cyclization can be used and were introduced in chapter 2.

\section{CXCR4 as the target of interest}

In chapter 6 we studied the C-X-C chemokine receptor type 4 (CXCR4) as a therapeutic target and aimed to design CXCR4 agonists based on the CXCR4-CXCL12 protein-protein complex using the workflow described in chapter 2. CXCL12 is the natural ligand of CXCR4, and it activates CXCR4 via binding to its extracellular domains. The neutrophil aging process is associated with vessel damage in an ischemia reperfusion model, with the CXCR4/CXCL12 chemokine receptor/ligand axis antagonizing this process and exerting cardioprotective functions ${ }^{26,}{ }^{27}$. Interactions between CXCR4 and CXCL12 inspired us to design CXCL12 mimics which bind and activate CXCR4 in a similar manner as CXCL12. This study starts with a retrieved model of full length CXCR4-CXCL1228. Key interactions that are located at the interfaces of the CXCR4-CXCL2 complex were collected from all relevant mutagenesis studies and potential hot spots were identified by the calculated per-residue energy contribution. The designed peptide comprised the essential parts of CXCL12 for binding and activating CXCR4 extracellularly and has been optimized by mutating specific residues such as to further improve binding affinity. The binding affinities of the designed peptides were estimated using the MM/PB(GB)SA method, with the most optimal peptide with the lowest binding free energy being selected for synthesis and biological activity testing.

Additionally, it was found that CXCR4 can be activated in an allosteric way ${ }^{29}, 30$. Therefore, we also decided to design a CXCR4 peptide agonist using its known allosteric agonist pepducin ATI-2341, derived from the intercellular loop of CXCR4, as the template. Due to the undefined binding site of ATI2341, the molecular docking method was employed to predict its binding mode in CXCR4 and generate a series of binding modes. To select the most likely binding mode close to the native binding mode, the MD simulation was combined with the BFE method to explore the stability of complexes and calculated BFEs between CXCR4 and ATI-2341. Furthermore, the designed intracellular peptides were optimized by introducing different types of linkers to improve binding affinity. The binding affinity was estimated in a similar way as described for the designed extracellular peptides.

In the end, the in vitro experimental test confirmed that all three selected peptides (both extracellular and intracellular peptides) showed activity in activating CXCR4 and limiting neutrophil aging in the blood stream. All in all, by following the workflow illustrated in chapter 2, we have successfully designed peptides that were able to activate CXCR4 and induce CXCR4/CXCL12 axis regulated signaling. This workflow can be performed in the future for designing peptidic modulators for similar GPCR proteins. 


\subsection{Challenges of structural bioinformatics tools}

Although we have successfully applied structural bioinformatics tools complementing experimental tools to characterize the protein structure-function relationships, protein dynamics, and protein-protein/ligand interactions in targeting CVD, these computational tools also have their limitations. An often-quoted statement about modelling techniques is that "all models are wrong, but some are useful", and structural bioinformatics are no exception to this. Structural bioinformatics is ultimately a tool based on in silico predictions, therefore the obtained results need to be confirmed and validated by biological experiments before further application. Nevertheless, structural bioinformatics approaches can guide scientific research forward as they can support and/or complement laboratory work.

A study using structural bioinformatics methods mostly relies on the 3D-structure of selected targets as it provides detailed structure-function information and determines the success or failure of structural bioinformatics. Therefore, targets with a reliable 3D structure are the basis for structural bioinformaticsbased studies. For targets without an experimentally determined 3D-structure nor reliable computational predicted model, the application of structural bioinformatics is limited.

The biological systems within the human body are highly complicated and fluctuating, their state is determined by many parameters, such as temperature, pressure, $\mathrm{pH}$, chemical reactions, ions and redox conditions, etc. Although efforts were made to make the MD simulation tool capable of simulating more parameters, it is still impossible to replicate an entire in vivo system.

The bigger the system to be studied, the longer the time required for MD simulation. For instance, a longer simulation (micro-millisecond) is needed to simulate biologically active processes such as enzyme catalysis, signal transduction, and allosteric regulation ${ }^{31}$. Despite the rapid development of computing power and improved efficiency of simulation algorithms, current MD simulation of protein systems is limited to the time scale of hundreds of nanoseconds. In order to reduce the running time, we commonly remove that part of the protein that is structurally non-related to the part of interest, without influencing the simulation results. As for example in chapter 5 , the $\mathrm{C} 1$ and $\mathrm{C} 2$ domain of FVa variants were removed during the MD simulation to accelerate the running speed in our study as these two domains are structurally non-related to the $\mathrm{A} 2$ domain. In addition, membranes were excluded for the optimization of our designed peptides in chapter 6 as similar-sized ligands binding to the same receptor are compared after a short MD simulation (several nanoseconds).

The scoring function in docking programs is commonly the approximation of the sum of various interactions. Significant improvement of docking accuracy can be obtained by training the scoring function with datasets containing a specific target, hence, it is expected that different scoring functions have advantages in calculating different interactions ${ }^{32-35}$. Therefore, it is important to overcome this inaccuracy and develop scoring functions that are robust towards non-specific classes of targets. 


\subsection{Future directions}

Today, artificial intelligence (AI) is being successfully applied to overcome challenging tasks in a large range of fields, thereby gaining a deeper awareness for scientists and researchers in drug discovery. Al's application in drug discovery and development ranges from protein structure prediction, target identification ${ }^{36,37}$, molecules design ${ }^{38,39}$ to its use in drug efficiency prediction and experimental design ${ }^{40}$. DeepMind's Al developed algorithm AlphaFold has beaten around 100 other computational approaches for the accurate prediction of the $3 D$ structure of proteins and its performance is equivalent to experimental methods in some cases but much faster ${ }^{41}$. The generation of AlphaFold represents a true breakthrough of $\mathrm{Al}$ in tackling scientific biological challenges. Additionally, increasingly the pharmaceutical industry is collaborating with Al-powered firms to drive their drug development processes in an efficient way, for instance, the partnership between Bayer and Exscientia, GSK and Insilico Medicine, Roche and GNS Healthcare. Although there are no Al-developed drugs on the market, the progress in pushing Al-generated candidates into clinical trials is underway and these drugs are expected to be marketed within the next decade. 


\section{References}

1. Mendis, S., et al., Global atlas on cardiovascular disease prevention and control / edited by: Shanthi Mendis ... [et al.]. World Health Organization: Geneva, 2011.

2. Yu, W.; MacKerell, A. D., Jr., Computer-Aided Drug Design Methods. Methods in molecular biology (Clifton, N.J.) 2017, 1520, 85-106.

3. Arita, K., et al., Structural basis for Ca2+-induced activation of human PAD4. Nature Structural \& Molecular Biology 2004, 11 (8), 777-783.

4. Muth, A., et al., Development of a Selective Inhibitor of Protein Arginine Deiminase 2. J. Med. Chem. 2017, 60 (7), 3198-3211.

5. Lewis, H. D., et al., Inhibition of PAD4 activity is sufficient to disrupt mouse and human NET formation. Nat Chem Biol. 2015, 11 (3), 189-191.

6. Linsky, T.; Fast, W., Mechanistic similarity and diversity among the guanidine-modifying members of the pentein superfamily. Biochimica et Biophysica Acta (BBA) - Proteins and Proteomics 2010, 1804 (10), 1943-1953.

7. Teo, C. Y., et al., Discovery of a new class of inhibitors for the protein arginine deiminase type 4 (PAD4) by structure-based virtual screening. BMC Bioinformatics 2012, 13 (17), S4.

8. Andrade, F., et al., Autocitrullination of human peptidyl arginine deiminase type 4 regulates protein citrullination during cell activation. Arthritis Rheum. 2010, 62 (6), 1630-1640.

9. Nomura, K., Specificity and mode of action of the muscle-type protein-arginine deiminase. Archives of Biochemistry and Biophysics 1992, 293 (2), 362-369.

10. Slack, J. L., et al., Autodeimination of protein arginine deiminase 4 alters protein-protein interactions but not activity. Biochemistry 2011, 50 (19), 3997-4010.

11. Land, H.; Humble, M. S., YASARA: A Tool to Obtain Structural Guidance in Biocatalytic Investigations. Methods Mol Biol 2018, 1685, 43-67.

12. Tan, K., et al., The structure of immunoglobulin superfamily domains 1 and 2 of MAdCAM- 1 reveals novel features important for integrin recognition. Structure 1998, 6 (6), 793-801.

13. Schreuder, M., et al., Blood coagulation factor Va's key interactive residues and regions for prothrombinase assembly and prothrombin binding. Journal of Thrombosis and Haemostasis 2019, 17 (8), 1229-1239.

14. Kalafatis, M., et al., The mechanism of inactivation of human factor $\mathrm{V}$ and human factor $\mathrm{Va}$ by activated protein C. J Biol Chem 1994, 269 (50), 31869-80.

15. Bos, M. H., et al., Venom factor $\mathrm{V}$ from the common brown snake escapes hemostatic regulation through procoagulant adaptations. Blood 2009, 114 (3), 686-92.

16. Lollar, P.; Parker, E. T., Structural basis for the decreased procoagulant activity of human factor VIII compared to the porcine homolog. J Biol Chem 1991, 266 (19), 12481-6.

17. Zollner, S., et al., Non-clinical pharmacokinetics and pharmacodynamics of rVIII-SingleChain, a novel recombinant single-chain factor VIII. Thrombosis Research 2014, 134 (1), 125-131.

18. Klamroth, R., et al., Comparative pharmacokinetics of rVIII-SingleChain and octocog alfa (Advate(®) ) in patients with severe haemophilia A. Haemophilia 2016, 22 (5), 730-8.

19. Seijkens, T. T. P., et al., Targeting CD40-Induced TRAF6 Signaling in Macrophages Reduces Atherosclerosis. J. Am. Coll. Cardiol. 2018, 71 (5), 527-542.

20. von Hundelshausen, P., et al., Chemokine interactome mapping enables tailored intervention in acute and chronic inflammation. Sci. Transl. Med. 2017, 9 (384).

21. Wichapong, K., et al., Structure-Based Design of Peptidic Inhibitors of the Interaction between CC Chemokine Ligand 5 (CCL5) and Human Neutrophil Peptides 1 (HNP1). J. Med. Chem. 2016, 59 (9), 4289-301.

22. Alard, J. E., et al., Recruitment of classical monocytes can be inhibited by disturbing heteromers of neutrophil HNP1 and platelet CCL5. Sci. Transl. Med. 2015, 7 (317), 317ra196.

23. Zarzycka, B., et al., Discovery of small molecule CD40-TRAF6 inhibitors. J. Chem. Inf. Model. 2015, 55 (2), 294-307.

24. Nicolaes, G. A., et al., Rational design of small molecules targeting the C2 domain of coagulation factor VIII. Blood 2014, 123 (1), 113-20.

25. Sperandio, O., et al., Identification of novel small molecule inhibitors of activated protein C. Thromb. Res. 2014, 133 (6), 1105-14.

26. Adrover, J. M., et al., A Neutrophil Timer Coordinates Immune Defense and Vascular Protection. Immunity 2019, 50 (2), 390-402.e10.

27. Hu, X., et al., Stromal cell derived factor-1 alpha confers protection against myocardial ischemia/reperfusion injury: role of the cardiac stromal cell derived factor-1 alpha CXCR4 axis. Circulation 2007, 116 (6), 654-63. 
28. Ziarek, J. J., et al., Structural basis for chemokine recognition by a G protein-coupled receptor and implications for receptor activation. Science Signaling 2017, 10 (471), eaah5756.

29. Janz, J. M., et al., Direct interaction between an allosteric agonist pepducin and the chemokine receptor CXCR4. J Am Chem Soc 2011, 133 (40), 15878-81.

30. Quoyer, J., et al., Pepducin targeting the C-X-C chemokine receptor type 4 acts as a biased agonist favoring activation of the inhibitory G protein. Proceedings of the National Academy of Sciences 2013, 110 (52), E5088.

31. Markwick, P. R. L.; McCammon, J. A., Studying functional dynamics in bio-molecules using accelerated molecular dynamics. Physical Chemistry Chemical Physics 2011, 13 (45), 20053-20065.

32. Seifert, M. H. J., Targeted scoring functions for virtual screening. Drug Discovery Today 2009, 14 (11), 562-569.

33. Kuenemann, M. A., et al., Which Three-Dimensional Characteristics Make Efficient Inhibitors of ProteinProtein Interactions? Journal of Chemical Information and Modeling 2014, 54 (11), 3067-3079.

34. Verdonk, M. L., et al., Virtual screening using protein-ligand docking: avoiding artificial enrichment. $J$ Chem Inf Comput Sci 2004, 44 (3), 793-806.

35. Logean, A., et al., Customized versus universal scoring functions: application to class I MHC-peptide binding free energy predictions. Bioorg Med Chem Lett 2001, 11 (5), 675-9.

36. Bakkar, N., et al., Artificial intelligence in neurodegenerative disease research: use of IBM Watson to identify additional RNA-binding proteins altered in amyotrophic lateral sclerosis. Acta Neuropathol 2018, 135 (2), 227-247.

37. Ferrero, E., et al., In silico prediction of novel therapeutic targets using gene-disease association data. Journal of Translational Medicine 2017, 15 (1), 182.

38. Kadurin, A., et al., druGAN: An Advanced Generative Adversarial Autoencoder Model for de Novo Generation of New Molecules with Desired Molecular Properties in Silico. Molecular Pharmaceutics 2017, 14 (9), 3098-3104.

39. Hartenfeller, M., et al., DOGS: Reaction-Driven de novo Design of Bioactive Compounds. PLOS Computational Biology 2012, 8 (2), e1002380.

40. Mak, K. K.; Pichika, M. R., Artificial intelligence in drug development: present status and future prospects. Drug Discov Today 2019, 24 (3), 773-780.

41. Callaway, E., 'It will change everything': DeepMind's Al makes gigantic leap in solving protein structures. nature 2020, pp 203-204. 



\section{Appendices}

Appendix I: English summary

Appendix II: Nederlandse samenvatting

Appendix III: Chinese summary

Appendix IV: Impact paragraph

Appendix V: Curriculum vitae

Appendix VI: List of publications

Appendix VII: Acknowledgment 



\section{Appendix I: English summary}

We aim to study cardiovascular diseases by performing a combination of in silico and in vitro approaches. Over the last two decades, structural bioinformatics provides various computational methods which are powerful and efficient in aiding and supporting experimental methods, and together they overcome the challenges that limit classical approaches. In this thesis, we have shown the successes of their cooperation by focusing on their application in several potential therapeutic targets of interest, including PAD4, coagulation factor Va and CXCR4.

Chapter 1 gives the general overview of this thesis. Frequently used computational tools are described in this chapter. The structure based virtual screening workflow is often used to discover the first-in-class small molecules towards specific target. For a protein without crystal structure, structural bioinformatics provides homology modeling method to predict its secondary structure. The molecular dynamic simulation method can simulate the movement of apo protein state in solvent or its complex with protein partner/inhibitor, followed by the calculation of binding free energies between two components to estimate their binding affinities. The important roles of therapeutic targets of interest in cardiovascular diseases were also described here.

Chapter 2 reviews how to rationally design modulators (activators or inactivators) that target (interrupt or stabilize) protein-protein interactions (PPIs). Protein-protein interactions have received increasing attention from researchers as they play important roles in different biological pathways. However, unlike a typical drug target with binding pocket, protein-protein interaction-based target has a flat and large surface which remain a challenge to be targeted by small chemical molecules. Thus, the workflow of rational design of peptides and peptidomimetics by the application of in silico approaches was described in this chapter in detail. The structure based peptidic modulators design requires the knowledge of 3D structure of the protein-protein complexes of targets, we thus summarized current experimental and computational methods used to determine 3D structure of protein-protein complexes.

Chapter 3 reviews the current understanding of PAD4 target, including the structural information and biological functions of PAD4, the crucial role of PAD4 in NET formation, and the role of PAD4 in the pathogenesis of rheumatoid arthritis, sepsis and thrombosis. PAD4 requires high concentration calcium to become active in vitro, however, such high concentration is not achievable in vivo. Thus, it remains unclear whether there are other cofactors which activate PAD4 and subsequently lows its requirement to calcium concentration. Although studies on the PAD4 and diseases are increasing, the mechanism of PAD4 activity control is still incomplete. As a promising therapeutic target, PAD4 has been investigated by focusing on the discovery of inhibitors by increasing studies, especially by Thompson's group. However, the overall high conservation in active site between different PAD isoforms forms a medicinal chemistry challenge with respect to the design of specific inhibitors. Finally, we compared and 
discussed PAD4 residues that interact with substrate or different types of inhibitors and give our input for the design of a novel class of therapeutics.

Chapter 4 studies the effect of autocitrullination on PAD4 activity by performing in vitro and in silico approaches. PAD4 can citrullinate itself, which is called autocitrullination or autodeimination. It remains controversial whether autocitrullination reduces PAD4 activity. We hypothesized that the autocitrullination is a self-control mechanism that controls PAD4 activity. To test our hypothesis, we initially generated citrullinated PAD4 by the incubation of PAD4 alone in the presence of calcium and observed 24/27 arginine citrullinated on PAD4 after 2 hours. PAD4 citrullinates itself in a time- and concentration-dependent manner. The activities of both PAD4 and citrullinated PAD4 were evaluated using small-, medium-, and big- sized substrates, and the obtained results showed that no significant difference detected between them. Furthermore, we performed molecular dynamic (MD) simulations to investigate structural dynamics and binding abilities of PAD4 and citrullinated PAD4 to their substrate. The MD simulation results indicated that autocitrullination does not change the shape of substrate binding pocket on PAD4. The calculated binding free energies suggested that PAD4 and citrullinated PAD4 exhibit comparable binding abilities with a H3-derived peptidyl substrate. Overall, our findings suggested that the autocitrullination does not represent a self-control mechanism for PAD4 activity.

Chapter $\mathbf{5}$ investigates the structural integrity of factor Va in venom of Pseudonaja textilis. The human coagulation factor $\mathrm{Va}(\mathrm{hFVa}$ ) become inactivated after activated protein $\mathrm{C}$ (APC)-catalyzed proteolysis. In contrast with this, the Pseudonaja textilis venom-derived factor $\mathrm{Va}(\mathrm{ptFVa})$ remains cofactor function despite APC cleavage. based on the structural analysis, hFVa contains one more cleavage site at position Arg306 and one less disulfide bond which covalently connect $\mathrm{A} 2$ and $\mathrm{A} 3$ domain compared with ptFVa. In order to uncover the mechanism principle which determines functional difference between $\mathrm{hFVa}$ and ptFVa, we experimentally created chimeric ptFVa by the addition of human Arg306 cleavage site and the deletion of disulfide bond, however, it still exhibits cofactor function. Furthermore, we in silico prepared $\mathrm{hFVa}$ and chimeric ptFVa structure and performed MD simulations to explore whether non-covalent interactions play a role in their structure-determined activities. The results observed by computational approaches showed that $\mathrm{hFVa}$ has a $\mathrm{A} 2$ domain dissociation trend along MD simulation time which was not observed in chimeric ptFVa. In addition, the calculated binding free energy between $A 2$ and $A 1+A 3$ domains in $h F V a$ increased significantly when comparing its values at $t=0 n s$ and $t=100 n s$. Finally, in silico approaches proposed a crucial loop which locates at A2 domain and could affects A2 domain association or dissociation state in ptFVa or hFVa.

Chapter 6 rationally designed various peptides which target C-X-C chemokine receptor type 4 (CXCR4) by the application of the workflow of rational design peptides based on PPIs using in silico approaches (as described in chapter 2). As CXCR4 is described as the major mediator for neutrophil aging, we aim to develop novel peptides as therapeutics which can activate CXCR4 and limit aged neutrophils for the high risk of patients with vascular diseases. We proposed two strategies by designing peptides which are supposed to bind CXCR4 intracellularly or extracellularly. We adopted a known pepducin and natural 
ligand CXCL12 as template through intracellular and extracellular designing path, respectively. All designed peptides were optimized through short MD simulations and their binding affinities to CXCR4 were estimated based on the binding free energy calculation. Finally, we have in total designed 75 peptides with selected three peptides displaying biological activities against CXCR4. Our presented methods here for peptide design can significantly reduce time and financial cost and can be used further for the development of peptidic modulators against other GPCR receptors.

Chapter 7 gives the critical discussion of the results obtained using the combination of computational and experimental approaches in this thesis. We also discussed the challenges and future development of the computational approaches. 



\section{Appendix II: Nederlandse samenvatting}

Het doel van het onderzoek, beschreven in dit proefschrift, is de bestudering van hart- en vaatziekten door toepassing van een combinatie van in silico en in vitro benaderingen. De afgelopen twee decennia heeft structurele bio-informatica verschillende computationele methodes opgeleverd die krachtig en efficiënt zijn en die experimentele benaderingen kunnen helpen en ondersteunen. Daarmee bieden ze een oplossing voor de beperkingen die klassieke onderzoeksmethodes met zich meebrengen. In dit proefschrift hebben we het succes van deze combinatie van methodes aangetoond door ons te concentreren op hun toepassing in verschillende potentiële therapeutische doelwitten, waaronder PAD4, stollingsfactor Va en CXCR4.

Hoofdstuk 1 geeft een algemeen overzicht van dit proefschrift. In dit hoofdstuk worden enkele van de meest gebruikte computationele methoden beschreven. De op structuur gebaseerde virtuele screening workflow wordt vaak gebruikt om kleine moleculen voor een specifiek doelwit te ontdekken. Voor een eiwit zonder bekende experimentele 3D structuur biedt structurele bioinformatica een op homologie gebaseerde modelleringsmethode om de secundaire structuur van een eiwit te kunnen voorspellen. De moleculair dynamische simulatiemethode kan de beweging van een apo-eiwit toestand in oplossing of zijn complex met een eiwit partner/remmer simuleren, gevolgd door de berekening van bindingsvrije energieën tussen twee componenten om zo hun bindingsaffiniteiten te kunnen schatten. De rol van therapeutische doelwitten die van belang zijn bij hart- en vaatziekten worden hier ook beschreven.

Hoofdstuk 2 bespreekt hoe men weloverwogen modulatoren (activatoren of inactivatoren) kan ontwerpen die zich richten op het onderbreken of stabiliseren van eiwit-eiwitinteracties. Eiwit-eiwit interacties hebben steeds meer aandacht gekregen van onderzoekers omdat ze een belangrijke rol spelen in verschillende biologische processen. In tegenstelling tot een typisch geneesmiddel doelwit met een bindingsplaats, heeft een op eiwit-eiwit interactie gebaseerd doelwit een groot en egaal oppervlak dat een uitdaging blijft om door kleine chemische moleculen te worden gebonden. Zo wordt de workflow van het rationeel ontwerpen van peptiden en peptidomimetica door de toepassing van in silico-benaderingen in detail beschreven in dit hoofdstuk. Het op structuur-gebaseerde ontwerp van peptidische modulatoren vereist de kennis van de 3D-structuur van de eiwit-eiwitcomplexen van onze doelwitten, dus hebben we de huidige experimentele en computationele methoden samengevat die worden gebruikt om de 3D-structuur van eiwit-eiwitcomplexen te bepalen.

Hoofdstuk 3 geeft een overzicht van de huidige kennis van het PAD4 eiwit, inclusief de structurele informatie en biologische functies, de cruciale rol van PAD4 in NET vorming, en de rol van PAD4 in de pathogenese van reumatoïde artritis, sepsis en trombose. PAD4 vereist een hoge concentratie calcium om actief te worden in vitro, maar een dergelijke hoge concentratie is in vivo niet haalbaar. Het blijft dus onduidelijk of er andere cofactoren zijn die PAD4 activeren en vervolgens de calciumdrempel verlagen. Hoewel het aantal gepubliceerde studies over het verband tussen PAD4 en ziekten toeneemt, is het 
exacte mechanisme van PAD4 activatie nog steeds onduidelijk. PAD4 staat daarnaast in de belangstelling als een veelbelovend therapeutisch doelwit door de ontdekking van PAD4 remmers, met name door de groep van Prof. Thompson in Worcester, Verenigde Staten. De algehele hoge conservering van de actieve site, het katalytische hart van het enzym, tussen verschillende PADisovormen vormt echter een uitdaging voor de medicinale chemie met betrekking tot het ontwerp van specifieke remmers. Ten slotte hebben we PAD4-residuen die een interactie aangaan met het substraat of verschillende soorten remmers vergeleken en besproken en hebben we voorstellen gegeven voor het ontwerp van een nieuwe klasse van therapieën.

Hoofdstuk 4 bestudeert het effect van autocitrullinatie op PAD4-activiteit door in vitro en in silico benaderingen uit te voeren. PAD4 is in staat zichzelf citrullineren, wat autocitrullinatie of autodeïminatie wordt genoemd. Het blijft controversieel of autocitrullinatie de PAD4-activiteit wel of niet vermindert. We veronderstelden dat de autocitrullinatie een zelfbeheersingsmechanisme is dat de PAD4-activiteit reguleert. Om onze hypothese te testen, hebben we in eerste instantie gecitrullineerd PAD4 gemaakt door de incubatie van enkel PAD4 in de aanwezigheid van calcium en vonden we na 2 uur dat 24 van de aanwezxige 27 arginine residuen gecitrullineerde waren. PAD4 citrullineert zichzelf op een tijds- en concentratie-afhankelijke manier. De activiteiten van zowel PAD4 als gecitrullineerde PAD4 werden geëvalueerd met behulp van kleine, middelgrote en grote substraten, en de verkregen resultaten toonden aan dat er geen significant verschil tussen beide soorten PAD4 kon worden aangetoond. Verder hebben we moleculaire dynamische (MD) simulaties uitgevoerd om de structurele dynamiek en bindingscapaciteiten van PAD4 en gecitrullineerd PAD4 aan hun substraat te onderzoeken. De MDsimulatie resultaten gaven aan dat autocitrullinatie de vorm van de substraat bindende pocket op PAD4 niet verandert. De berekende bindingsvrije energieën suggereerden dat PAD4 en gecitrullineerd PAD4 vergelijkbare bindingscapaciteiten vertonen met een van $\mathrm{H} 3$ afgeleid peptidylsubstraat. In het algemeen suggereerden onze bevindingen dat de autocitrullinatie geen zelfbeheersingsmechanisme van PAD4activiteit vertegenwoordigt.

Hoofdstuk 5 onderzoekt de structurele integriteit van factor Va in het gif van de slang Pseudonaja textilis. De menselijke stollingsfactor Va (hFVa) wordt geïnactiveerd na geactiveerde proteïne C (APC)gekatalyseerde proteolyse. In tegenstelling hiermee blijt de van Pseudonaja textilis afkomstige factor $\mathrm{Va}$ (ptFVa) cofactorfunctie ondanks APC-splitsing behouden. Op basis van de structurele analyse bevat hFVa in vergelijking met ptFVa één splitsingsplaats op positie Arg306 en één disulfide binding minder die het A2- en A3-domein covalent verbindt. Om het mechanistisch principe te ontdekken dat het functionele verschil tussen hFVa en ptFVa verklaart, hebben we experimentele chimeren van ptFVa gemaakt door toevoeging van de menselijke Arg306-splitsingsplaats en de verwijdering van disulfide bindingen; echter deze chimeren vertonen, na splitsing door APC, nog steeds een cofactorfunctie. Verder hebben we in silico de hFVa- en chimera ptFVa-structuur gemodelleerd en MD-simulaties uitgevoerd om te onderzoeken of niet-covalente interacties een rol spelen in hun structuur-bepaalde activiteiten. De resultaten die werden verkregen door computationele benaderingen toonden aan dat hFVa een neiging tot dissociatie van het A2-domein heeft langs de MD-simulatietijd die niet werd 
waargenomen in chimera ptFVa. Bovendien nam de berekende vrije bindingsenergie tussen A2- en $A 1+A 3-d o m e i n e n$ in $h F V a$ significant toe bij vergelijking van de waarden op $t=0$ ns en $t=100 n s$. Ten slotte stelden in silico-benaderingen een cruciale lus voor die zich op het A2-domein lokaliseert en de associatie- of dissociatiestatus van het A2-domein in ptFVa of hFVa zou kunnen beïnvloeden.

Hoofdstuk 6 beschrijft het rationele ontwerp van verschillende peptiden die zich richten op de C-X-C chemokine receptor type 4 (CXCR4). Dit ontwerp is gedaan door toepassing van de workflow voor het rationeel ontwerpen van peptiden op basis van eiwit-eiwit interacties met behulp van in silicobenaderingen (zoals beschreven in hoofdstuk 2). Aangezien CXCR4 wordt beschreven als de belangrijkste mediator voor veroudering van neutrofielen, streven we ernaar nieuwe peptiden te ontwikkelen als therapieën die CXCR4 kunnen activeren en veroudering van neutrofielen kunnen terugdringen in patiënten met een verhoogd hoge risico op hart- en vaatziekten. We hebben twee strategieën voorgesteld door peptiden te ontwerpen waarvan wordt verondersteld dat ze intracellulair of extracellulair aan CXCR4 binden. We hebben een bekend pepducine en natuurlijke ligand CXCL12 als sjabloon aangenomen via respectievelijk een intracellulaire en extracellulaire ontwerproute. Alle ontworpen peptiden werden geoptimaliseerd door middel van korte MD-simulaties en hun bindingsaffiniteiten voor CXCR4 werden geschat op basis van de berekening van de bindingsvrije energie. Tot slot hebben we in totaal 75 peptiden in silico ontworpen waarvan uiteindelijk drie geselecteerde peptiden zijn gesyntheseerd en waarvan de biologische activiteit tegen CXCR4 is getest, en die activiteit vertonen. De hier gepresenteerde methoden voor peptideontwerp kunnen de benodigde tijd en financiële kosten aanzienlijk verminderen en verder worden gebruikt voor de ontwikkeling van peptidische modulatoren tegen andere GPCR-receptoren.

Hoofdstuk 7 geeft een kritische bespreking van de resultaten die zijn verkregen in dit proefschrift met de combinatie van computationele en experimentele benaderingen. We bespreken ook de uitdagingen en toekomstige ontwikkelingen van de beschreven computationele methodes. 



\section{Appendix III: Chinese summary}

过去的二十年中, 结构生物信息学提供了多样化的计算和模拟方法, 尤其是在帮助和支持实验方法上, 显示了非常有效且强大的功能。同时, 采用计算模拟和实验相结合的方法可以解决一些传统实验不能解 决的问题和挑战。在本论文中, 通过研究心血管疾病潜在的治疗靶点, 蛋白质精氨酸脱亚胺酶 PAD4, 凝血因子 FVa，以及趋化因子受体 CXCR4，展示了计算模拟和实验方法相结合的成功应用。

第一章给出了整篇论文的概括, 即采用计算模拟和实验相结合的方法来进行心血管疾病的研究。同时介 绍了常用的结构生物信息学工具及其应用, 例如, 基于蛋白结构的虚拟篮选通常用于发现针对特定靶点 的新型小分子化合物；对于一个没有蛋白结构的靶点来说，结构生物信息学会提供同源建模的方法来预 测它的二级结构; 分子动力学可以模拟单个蛋白或者蛋白与其底物或抑制剂在溶剂中的运动状态, 通过 计算自由结合能来预估两个分子之间的结合力。最后, 本章还介绍了论文中所研究的几个靶标蛋白在心 血管疾病中的重要作用。

第二章回顾了如何根据蛋白质-蛋白质之间的相互作用来合理设计调节剂, 包括抑制剂和激活剂, 它们 分别阻断和稳定蛋白质-蛋白质之间的相互作用。蛋白质-蛋白质之间的相互作用在不同的生物学通路中 发挥着重要的作用, 因此受到研究人员越来越多的关注。。然而, 与具有结合口袋的典型药物靶点不同, 基于蛋白与蛋白相互作用的靶标具有平坦且较大的表面积, 因此通过设计小分子化合物来针对这类靶标 仍然具有很大的挑战。本章内容详细描述了通过计算机的方法来合理设计肽和肽的类似物的工作流程。 此外, 基于结构的肽类调节剂的设计首先需要了解目标蛋白质与蛋白质复合物的 3D 结构, 因此我们还 总结了当前的一些实验和计算方法, 用于确定蛋白质与蛋白质复合物的 3D 结构。

第三章回顾了目前对于 PAD4 这个靶点的理解, 包括 PAD4 的结构信息和生物学功能, PAD4 在中性粒 细胞陷阱形成过程当中的重要作用, 以及 PAD4 在类风湿性关节炎, 败血病, 血栓形成和发病过程中的 重要作用。关于 PAD4 跟疾病相关性的研究逐渐增多, 但是 PAD4 的活性控制机理始终是不完善的。作 为一个有希望的靶标, 更多的研究专注于寻找能抑制 PAD4 活性的抑制剂, 特别是 Thompson's 的研究 
小组。然而, PAD 蛋白家族内部成员之间具有高度的同源性, 特别是在活性口袋的部位, 从药物化学角 度来讲，这对于找到针对 PAD4 有较高选择性的抑制剂有一定的挑战。因此，我们比较并讨论了 PAD4 结构上能与底物和不同类型的抑制剂有特异性结合的氨基酸, 从而为设计新型的且针对 PAD4 有较高选 择性的抑制剂提供意见。

第四章采用计算模拟和体外实验相结合的方法研究了 PAD4 自我瓜氨酸化对于其活性和功能的影响。目 前, 对 PAD4 的自我瓜氨酸化是否减弱其自身活性是有争议的。我们的假设是自我瓜氨酸化是 PAD4 的 一个自我活性控制机制。为验证该假设, 我们通过对钙离子激活后的 PAD4 进行单独孵育, 产生了自我 瓜氨酸化的 PAD4, 在两个小时以后观查到了 PAD4 结构上的 27 个精氨酸中有 24 个被自我催化变成了 瓜氨酸, 且 PAD4 对其自身的瓜氨酸化呈现出对浓度和时间的依赖性。通过催化不同大小的底物, 我们 对 PAD4 和瓜氨酸化的 PAD4 的活性进行了评估, 所得的结果表明它们的活性之间没有显著化的差异。 此外, 我们还通过分子动力学模拟研究了 PAD4 和瓜氨酸化的 PAD4 的结构的稳定性以及分别跟底物的 结合能力。结果表明自我瓜氨酸化不会改变 PAD4 的结合口袋的构型。通过自由结合能的计算结果, 显 示了 PAD4 和瓜氨酸化的 PAD4 展现出对同一衍生肽底物相当的结合能力。综上所述, 我们的研究结果 表明了自我瓜氨酸化并不能代表 PAD4 活性的自我控制机制。

第五章内容研究凝血因子 FVa在澳大利亚蛇毒液中结构的完整性。人的凝血因子 FVa 被活性蛋白酶 APC 水解切割后失活。截然相反的是, 在澳大利亚蛇毒中的同源蛋白 FVa 在经过 APC 水解切割后依然保持 活性。基于它们结构分析发现, 相比于蛇毒中的凝血因子 FVa，人的凝血因子 FVa 多了一个切割位点, 少了一个跨结构域的二硫键。为了验证这些结构的差别对于它们被切割后功能的影响, 我们先通过实验 对蛋白结构进行了改造, 使得改造后的蛇毒 FVa 携带与人类 FVa 相同数目的切割位点, 并且其跨结构 二硫键被阻断。然而, 实验结果显示改造后的蛇毒 FVa 依旧具有凝血功能。进一步地, 我们采用计算的 方法对其进行研究。我们分别对人类的和改造后的蛇毒 FVa 结构进行了分子动力学模拟, 去探索非共价 键的结合是否对于蛋白被切割后的结构和功能有影响。计算结果表明人类 FVa 结构上的 A2 结构域随着 模拟时间的延长有从整体结构中解离的趋势, 这在蛇毒 FVa 中未观察到。此外, 当比较 A2 结构域与 $A 1+A 3$ 结构域之间的自由结合能在模拟时间 $t=0 n s$ 和 $t=100 n s$ 的差异时, 计算的自由结合能显示 $A 2$ 的 
结合力在人类的 FVa 中显著下降。最后, 计算结果还揭露出了一个位于 A2 结构域上的重要的 loop 区, 它可能对 A2 结构域在蛇毒 FVa 或人类 FVa 中的关联或解离状态起着至关重要的作用。

第六章合理设计了针对 CXCR4 靶蛋白的肽类调节剂。实验方法遵循我们在第二章中所叙述的基于蛋白 与蛋白之间的相互作用来设计调节剂的工作流程。CXCR4 是中性粒细胞衰老的主要调控者。我们的目 标是针对 CXCR4 设计新的肽来作为治疗剂, 这些肽可以激活 CXCR4 并限制衰老的中性粒细胞的数量, 从而降低心血管疾病患者的高风险。设计过程中, 我们提出了两种策略, 即分别以 pepducin 和天然的 配体 CXCL12 作为模板, 设计出来的肽通过胞内和胞外来结合 CXCR4。所有设计的肽都通过分子动力 学模拟进行了优化, 并根据计算的自由结合能评估了它们对 CXCR4 的结合力。我们总共设计了 75 种 肽, 并选择了其中结合力最好的三种肽进行生物学实验验证其活性。实验结果表明三种肽均能对 CXCR4 显示出不同程度的生物学活性。因此, 我们在此章介绍的肽的设计方法可以显著减少时间和财 务成本, 并可进一步用于开发针对其他 GPCR 受体的肽调节剂。

第七章辩证地讨论了使用计算和实验方法的结合在本论文研究中所获得的结果。此外, 我们还讨论了计 算方法所面临的挑战以及对未来的展望。 



\section{Appendix IV: Impact paragraph}

The objective of this thesis. In this thesis, we have applied various structural bioinformatics tools in combination with wet-lab experiments to investigate the mechanism of action of (selected) proteins involved in thromboinflammation. Also, we have employed these approaches for structure-based molecular design to develop bioactive compounds to modulate inflammation. Structural bioinformatics can bring insight into protein structures and protein-protein/ligand interactions at the atomic level. With the advancement of technologies and the increase in computer power, structural bioinformatics increasingly can help researchers to solve complex problems while saving considerable time and cost in biomedical science.

How does structural bioinformatics help researchers in modern drug discovery? Drug discovery is a very time- and money-consuming process. The concise workflow of drug discovery includes target identification, hit identification, lead optimization, preclinical trials and clinical trials, drug approvals. Structural bioinformatics have made key contributions to the early drug discovery process by offering a wide variety of different tools and technologies. Some of these tools were successfully used in this thesis. For instance, an homology modeling approach was used to build a three-dimensional (3D) model structure of the target protein based on knowledge of its primary sequences and of the 3D structure of a template protein. By taking advantages of available 3D structures and bioinformatic tools, researchers can explore the ligand binding site, the flexibility of a binding pocket and also protein-ligand interactions. Additionally, when crystalized or homology modelled 3D structure of target protein are available, researchers can perform a molecular docking approach to obtain protein-ligand/drug complexes and estimate binding affinity of ligands. However, the application of molecular docking is not only limited to predict possible ligand binding conformations. Docking is also widely conducted to screen for potential (drug) candidates binding to a specific target from a database containing large numbers of drug-like compounds. QSAR (Quantitative Structure and Activity Relationships) approach is applied to characterize and investigate a correlation between the physico-chemical features of molecules and their experimental biological activities. With the purpose of lead optimization, such relationship between molecular structure and activity can be employed to predict the activity of new designed molecules. Molecular dynamics (MD) simulation approach monitors protein motions in a semi-physiological environment by adding solvent and ions or membranes for transmembrane proteins to a protein structure in silico. By analyzing molecular dynamics trajectories, researchers can investigate the structural stability of a target protein, an allosteric pocket, and key residues between proteinprotein/protein-ligand complex. Moreover, MD trajectories can also be utilized for binding free energy (BFE) calculations to predict and prioritize binding affinity of candidate compounds which can help to reduce the number of compounds that will be subjected to experimental testing, in order to save cost and time. 
How do structural bioinformatics impact cardiovascular diseases (CVDs)? With the development of computational power, structural bioinformatic tools have been widely used in a broad range of researches, including recently emerging topics like COVID-19 ${ }^{1}$. Here, we have chosen several potential therapeutic targets implicated in CVD, particularly to target proteins involved in thromboinflammation which are PAD4, coagulation factor $V$ and CXCR4. The American Heart Association reported that $\sim 48 \%$ of the total population in American adults have some type of cardiovascular disease and CVD is the number one cause of death for people in US and worldwide ${ }^{2}$. Although increasing biological pathways are revealed and characterized in cardiovascular diseases through disease biology, the detailed mechanisms of action of proteins involved in pathways is hardly disclosed by only relying on wet-lab techniques. Structural Bioinformatics can assist here and can guide and rationalize experimental studies. With the help of computational methods, it becomes achievable to uncover possible mechanisms and provide novel therapeutics towards specific disease.

For example, in Chapter 4, we studied whether autocitrullinated PAD4 has enzymatic activity by simulating PAD4 and autocitrullinated PAD4 structures using molecular dynamic (MD) simulations. The obtained computational results were consistent with obtained experimental results and further explained what has been displayed in experimental outcomes. Another example is the study of coagulation factor FVa target in human plasma ( $\mathrm{hFVa}$ ) and in the venom of pseudonaja textilis (ptFVa) described in Chapter 5. hFVa structure lost its activity after cleavage by activated protein C (APC); in contrast, the homologous FVa protein in the venom of pseudonaja textilis showed complete activity even after APC cleavage. Through wet-lab approaches, it was confirmed that none of the structural elements (disulfide bond across different domains, the number of cleavage sites) play a role in preserved activity in ptFVa after proteolysis by APC. However, it was challenging to further explore what structural features then determine the observed activity differences by only relying on wet-lab techniques. Therefore, we decided to employ computational methods to investigate APC cleaved hFVa and the ptFVa structure. MD simulations showed a trend towards A2 domain dissociation in hFVa but not in ptFVa, which is consistent with experimental results. Furthermore, the computational methods uncovered the presence of a key region which possibly plays a crucial role in the structural integrity of ptFVa. Details of this need to be verified by further experiments. In Chapter 6, we have applied computer- and structure-based methods to design CXCR4 agonists. By application of these approaches, we were able to select three peptides from more than trillion candidates available in chemical space for experimental testing of their agonistic activity in in vitro and in vivo experiments. Importantly, the most potent peptide exhibited therapeutic benefits to protect mice from myocardial death during acute-myocardial infarction. Obviously, as demonstrated in Chapter 6, we can accelerate the drug discovery process by use of structural bioinformatic methods to identify "lead" compounds which can be further improved, optimized and tested in different pre- and clinical phases and finally developed into drugs for CVDs.

Who gains benefits from structural bioinformatics tools practically? Increasingly pharmaceutical companies apply computational approaches into drug design and discovery pipelines. The pharmaceutical industry employs computational chemists to work with medicinal chemists to accelerate 
the drug discovery process. Some examples ${ }^{3}$ are listed in Table 1 to show successful applications of computer-aided drug discovery. Additionally, pharmaceutical companies engage in more relationships with Al-derived biotech companies. For example, Sumitomo Dainippon Pharma announced collaboration with Exscientia and their research alliance resulted in the discovery of DSP-1181 which has entered Phase I clinical trial in 20204. Another recent example is during COVID-19 period start-up company BenevolentAI screened approved drugs for treating COVID-19, several promising candidates were identified only within several days, with baricitinib (Eli Lilly's Olumiant) approved for treating COVID-19 in the USA and EU5 ${ }^{5}$. Conclusively, this trend is expected to be continued since a drug can be brought from its concept into clinical trials much more cost-effective and faster with the assistance of computational approaches. Not only pharmaceutical companies who gain profits from these approaches and methods. In the long run medical doctors, patients, and whole societies will also gain benefits (both economical and by improved public health) from an improved development of new potent drugs. These novel drugs can be used to save or improve patients' life which will help to reduce the number of patients admitted to hospital and save treatment cost.

Table 1. Examples of drugs ${ }^{3}$ that are approved for use with the assistance of computer methods.

\begin{tabular}{|c|c|c|c|c|}
\hline Drug & Structure & Target protein & Use & Company \\
\hline Captopril & & $\begin{array}{c}\text { angiotensin } \\
\text { converting } \\
\text { enzyme inhibitor }\end{array}$ & $\begin{array}{l}\text { hypertension } \\
\text { and heart } \\
\text { failure }\end{array}$ & $\begin{array}{c}\text { Bristol Myers- } \\
\text { Squibb }\end{array}$ \\
\hline Dorzolamide & & $\begin{array}{c}\text { carbonic } \\
\text { anhydrase } \\
\text { inhibitor }\end{array}$ & glaucoma & Merck \\
\hline Saquinavir & & $\begin{array}{l}\text { HIV-1 protease } \\
\text { inhibitor }\end{array}$ & HIV-1 & Roche \\
\hline Zanamivir & & $\begin{array}{c}\text { sialic acid- } \\
\text { analogue } \\
\text { neuraminidase } \\
\text { inhibitor }\end{array}$ & $\begin{array}{l}\text { influenza } A \\
\text { and } B \text { virus }\end{array}$ & $\begin{array}{c}\text { Gilead } \\
\text { Sciences }\end{array}$ \\
\hline Aliskiren & & $\begin{array}{c}\text { active } \\
\text { nonpeptide } \\
\text { renin inhibitor }\end{array}$ & hypertension & Novartis \\
\hline Boceprevir & & $\begin{array}{c}\text { hepatitis } C \text { virus } \\
\text { NS3/4A } \\
\text { protease } \\
\text { inhibitor }\end{array}$ & $\begin{array}{l}\text { chronic } \\
\text { Hepatitis C } \\
\text { genotype } 1 \\
\text { infection }\end{array}$ & $\begin{array}{l}\text { Schering- } \\
\text { Plough }\end{array}$ \\
\hline
\end{tabular}


New developments. Inherently to the nature of computational methods, artificial intelligence (AI) is expected to have an impact on future structural bioinformatics and computational drug design. Al has already been widely used in virtual screening, QSAR, de novo molecular design and even clinical trial prediction. It is expected to be increasingly applied into more directions in drug discovery with more advanced algorithms and more powerful computing ability.

\section{References}

1. Waman, V. P., et al., The impact of structural bioinformatics tools and resources on SARS-CoV-2 research and therapeutic strategies. Brief Bioinform 2021, 22 (2), 742-768.

2. Virani, S. S., et al., Heart Disease and Stroke Statistics-2020 Update: A Report From the American Heart Association. Circulation 2020, 141 (9), e139-e596.

3. Talele, T. T., et al., Successful applications of computer aided drug discovery: moving drugs from concept to the clinic. Curr Top Med Chem 2010, 10 (1), 127-41.

4. Burki, T., A new paradigm for drug development. Lancet Digit Health 2020, 2 (5), e226-e227.

5. Stebbing, J., et al., Mechanism of baricitinib supports artificial intelligence-predicted testing in COVID-19 patients. EMBO Mol Med 2020, 12 (8), e12697. 


\section{Appendix V: Curriculum vitae}

Xiaosong Liu was born on the $13^{\text {th }}$ of October in 1992 in Jining city, Shandong province, China, where she grew up. In September 2010, she started her college study in Bioengineering at Ludong University, Yantai city, Shandong province and obtained Bachelor's degree in June 2014. In the same year of September, she continued majoring in Biophysics at China Agricultural University in Beijing and obtained the degree of Master of Science in June 2016. During master's study, Xiaosong explores the stable compounds in inorganic $\mathrm{Mg}-\mathrm{Cl}$ system at high pressure using computational methods. In October 2016, she has been awarded four-year national scholarship from China Scholarship Council (CSC) and moved to the Netherlands to start her $\mathrm{PhD}$ training at department of Biochemistry, Cardiovascular Research Institute Maastricht (CARIM), Maastricht University. Since then, she focused on structure

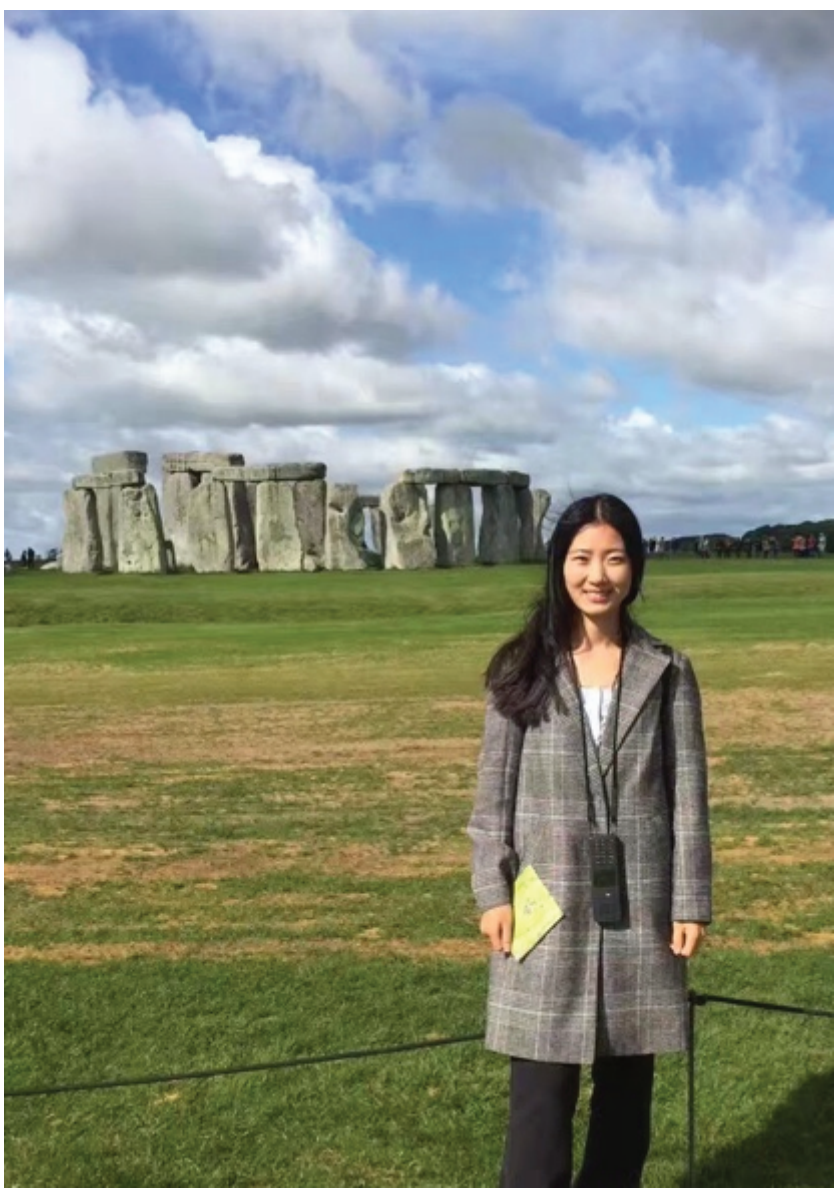
based early drug design and discovery using the combination of computational modeling approaches and wet lab-based methods towards several thromboinflammation targets. Her research described in this thesis was supervised by assoc. prof. Gerry Nicolaes, prof. Chris Reutelingsperger and dr. Kanin Wichapong. In December 2020 she returned to China and started working as computational chemist at Insilico Medicine company in Shanghai since April 2021. 



\section{Appendix VI: List of publications}

\section{Full Papers}

- Wichapong K, Poelman $\mathrm{H}^{*}$, Ercig $\mathrm{B}^{*}$, Hrdinova $\mathrm{J}^{*}$, Liu $\mathbf{X}^{*}$, Lutgens E, Nicolaes GAF, Rational modulator design by exploitation of prote-protein complex structures. Future Med Chem. 2019; 11(9):1015-1033.

- Bohler S, Liu X, Krauskopf J, Caiment F, Aubrecht J, Nicolaes GAF, Kleinjans JCS, Briedé JJ, Acetaminophen Overdose as a Potential Risk Factor for Parkinson's Disease. Clin TransI Sci. 2019; 12(6): 609-616.

- Liu X, Wichapong K, Lamers S, Reutelingsperger CPM, Nicolaes GAF, Autocitrullination of PAD4 does not alter its enzymatic activity with substrate: in vitro and in silico studies. Int. J. Biochem. Cell Biol. 2021; 134: 105938.

- Liu X*, Arfman T*, Wichapong K, Reutelingsperger CPM, Voorberg J, Nicolaes GAF, PAD4 takes charge during neutrophil activation: impact of PAD4 mediated NET formation on immune-mediated disease. J. Thromb. Haemost. 2021; 19(7): 1607-1617.

- Schreuder $\mathrm{M}^{*}$, Liu X*, Cheung K, Reitsma P, Nicolaes GAF, Bos MHA, Pseudonaja textilis venomderived factor Va retains structural integrity following proteolysis by activated protein $C$. Arterioscler. Thromb. Vasc. Biol. 2021;41(8):2263-2276.

- Liu X, Aroca-Crevillen A, Reutelingsperger CPM, Hidalgo A, Nicolaes GAF*, Wichapong K*, Rational design of peptide agonists activating $C-X-C$ chemokine receptor type 4 (CXCR4). (In preparation)

\section{Conference abstracts}

- Liu X, Wichapong K, Reutelingsperger CPM, Nicolaes, Identification of novel PAD4 inhibitors by application of in silico and in vitro screening. Netherlands Society on Biomolecular Modelling (NSBM) Fall meeting 2017, Utrecht, The Netherlands

- Liu X, Wichapong K, Reutelingsperger CPM, Nicolaes GAF, Identification of novel PAD4 inhibitors by application of in silico and in vitro screening. BioSB 2017, (oral presentation) De Werelt, Lunteren, The Netherlands

- Liu X, Wichapong K, Lamers B, Reutelingsperger CPM, Nicolaes GAF, The Effect of Autocitrullination on Human Peptidyl Arginine Deiminase 4 Activity. Netherlands Society on Biomolecular Modelling (NSBM) Fall meeting 2018, Utrecht, The Netherlands

- Liu X*, Schreuder M*, Cheung K, Reitsma P, Bos MHA, Wichapong K, Nicolaes GAF, Applications of computational methods to gain insight into the structural integrity of human and Pseudonaja textilis coagulation factor V. 3D-Biolnfo Community Annual meeting, 2020, Online

\section{*These authors contributed equally}





\section{Appendix VII: Acknowledgment}

I really enjoyed the past four years at Biochemistry department. I want to express my thanks to following people who helped me with my $\mathrm{PhD}$ study and who made my life enriching.

My promotor and supervisor Dr. Gerry Nicolaes, dear Gerry, who interviewed me and invited me to Netherlands for study. I learned to face unexpected results positively and to face satisfying results critically from you when doing research. Such truly attitude to science is much valuable than any scientific achievements. Your networks in academic field offered me great opportunity to work on different projects and in collaboration with different groups. Out of science, you organized very enjoyable group events (escape room, international dinners, Witloof beer tour, afterwork drinks) which left precious memories to me. It was a great pleasure to have dinner with you and your family at your place. I am extremely grateful to all of this. I wish all the best to you and your family!

My co-promotor Prof. Dr. Chris Reutelingsperger, dear Chris, you always try to help me solve problems during weekly meetings. Thank you very much for your constructive feedback in experimental part and thank you for spending time to check my manuscripts and chapters.

My co-promotor and daily supervisor Dr. Kanin Wichapong, dear Kanin, you guided me into computational chemistry field and taught me all related computational skills with patients. I can always come to you when I have questions and doubts. Your encouraging words revived my drooping spirits and kept me forward. When I first arrived at Maastricht, as Asian, you understood my culture shock and assisted me to adapt the Western culture, which indirectly helped my PhD study by making a comfortable environment. Thank you very much for your company and support all along. Best wishes to your future world journey! Welcome to China again!

I would like to take this opportunity to acknowledge all members of the committee, Prof. Dr. Casper G. Schalkwijk (Maastricht University), Dr. Ingrid Dijkgraaf (Maastricht University), Dr. Pablo Garcia de Frutos (University of Barcelona, Spain), Prof. Dr. Guido R.M.M. Haenen (Maastricht University), and Prof. Dr. Wim Vranken (Free University of Brussels, Belgium), thank you all very much for reviewing and approving my thesis.

I would like to extend my thanks to China scholarship Council (CSC) for awarding financial support to my four-year PhD study.

I thank all co-authors for making significant contributions to the chapters in this thesis. Mark and Mettine, it was a great pleasure to work with you, I learned a lot about coagulation factor $\mathrm{V}$. Tom, thanks for your efforts on PAD4 review. It was a busy day to purify PAD4 when you came to Maastricht. Hopefully, the BBQ we had at Hessel's backyard left nice memories to you. Jan, I was glad to meet you 
when you came to Maastricht for visiting our group. Thank you for commenting and revising the manuscript of PAD4 review.

Here, I also would like to take this opportunity to thank Prof. Dr. Gerd Vriend, at faculty of Medical Sciences, Radbound University Nijmegen. Dear Gerd, you are an expert in structure bioinformatics filed. It was a great opportunity for me to join your classes and learn structure bioinformatics knowledge from you. Back then, thank you for offering guest house for me (and Angelina) and thank your wife for cooking delicious food for us. I wish you, your wife and your little son all the best!

Dear Prof. Dr. Tilman Hackeng, the head of Biochemistry. I like your talented speeches. We have many excellent events going on at our department, the Tilman's backyard Party in September is definitely the one I always look forward to joining every year. Thank you for creating a very comfortable environment for all researchers. I am very proud of being a member of Biochemistry (the best department). By the way, I remember that I still owe you a Korean Tteok-bokki (spicy rice cake) recipe.

Danielle and Hessel, thank you for being my virtual remote paranymphs. Danielle, "my princess", thank you very much for making time to check my chapters. All the nice time we had in past years made us to be friends. You share your $\mathrm{PhD}$ experience with me and encourage me to step out. You have talent of organizing activities, connecting people together and warming up quiet atmosphere. I also like another side of you (after drinks ...). You are always welcome to China and I will be very happy to accommodate you there. Hessel, it was enjoyable time when we sit at the same office room despite "endless debates" between us. By the way, thank you for organizing and hosting events at my place without my permission (just kidding). As a friend, I know you have friendly and creative personality. Thanks for all your help in my personal life. Success with your remaining PhD! And no more password set as "Song123"! Johana, thanks for coming to Maastricht so that I had the opportunity to know you as a friend. You are a friendly, social and smart person. We could share all kinds of emotions and do crazy things together. We had so much fun in boat parties, winter ball party, game night, girl's night, Tea Zone, ... Thanks for allowing me to stay at your place when I lost my key. I will miss langoše and vánočka you made in house. Best wishes to you and Pavel in Czech! Angelina, it must be a special fate that we started the PhD around the same time. We had great fun at museum night, cycling to Liège, visiting Liège market on Sunday morning, sharing good cosmetics, ... I knew you have experienced difficult time with your PhD, I wish I could have more conversations with you. Success with your PhD! Bogac, your attitude to both life and science is always calm and steady, which gives people a sense of security. Thank you for making an opportunity for me to join TEDx Maastricht, it was a great gift to my birthday. Joram, thank you for managing our lab! You started your PhD later than all of us but supervised the most students. You are always willing to help. Sorry that if I disturbed you in the office when "debating" with Hessel. Femke, thank you for staying with us in the group. We had great fun with girl's night, Secret Santa at your place. Thank you and your boyfriend for inviting me to your place to enjoy the great Dutch food night. Success with your coming PhD challenge! Roy, thanks for teaching me lab skills. For me, our lab is still "Roy's lab". Also, thanks for fixing my secondhand bedframe. We had nice conversation when you 
"accompanying me" on the way to home after party. Thank you for sharing the home-made kimchi with me. I know that you like different kinds of food, you are always welcome to china to enjoy all kinds of dishes.

Dear Stella, in biochemistry, we all know burger, bitterball, and Stella. You are like an angel who tries to make everyone delighted. It is artistic to have all alcoholic bottles in the lab for decoration. Besides, it was so nice to join the Friday afternoon drinks. You know everything and everyone in Maastricht though you are Belgian. And you even know some Chinese who I don't know at all. It was so relaxed and happy to share a lot of time together chilling with you in Maastricht. As the picture you sent to me, adult friendships are hard, I put our friendship in heart. I will be thinking of you, you are a strong woman, and you will fight all the bad things, hope all coming is well! Betta, thanks for your "secret" help. I very much enjoyed our casual but profound chat about life. Peter, we have watched the ending of "Games of Thrones" at Van Gosh meeting room together, which was enjoyable. David, I enjoyed the polish soup you made at International Dinner. Good luck with rest of your PhD and future career! Stijn, thanks for organizing gables, I enjoyed a lot though I never win. I also brought the cooking apron of " $\mathrm{C}^{3} \mathrm{D}$ (chocolate chip cookie dough) Team" back to China. Alice, we are the ones who are always struggling with choosing food for lunch at hospital Canteen. Tom, thanks for your Secret Santa gift, I offer you delicious sushi meal when you come to China. Ingrid, I always see you when I work late in the department, thanks for chatting with me sometimes. Rory, it's pity that we didn't have chance to work together. Thanks for your suggestions and tips about PAD4 purification. Anouk, you are always so friendly, I wish you all the best! Hans, thanks for introducing your fashionable and intriguing arts to me. It was always nice to talk with you when working at cold NMR room. Alexandra, Dennis, Stepan, Adash, Danique, Annemiek, Stan, I enjoyed the time with all of you as colleagues in the same department.

Dear Trees and Lidewij, thank you for arranging me in Biochemistry and dealing with all documents related to me. Trees, you are like a "Mum" in the department, I like you sharing your travel experience in China with me though it was 20 years ago. Lidewij, we had great fun with our "Dough team" in Biochemistry Dayout.

Gina, I enjoyed the real Italian pizza Matteo made. I felt so relax and happy when chilling together with you after work. Also, I very much enjoyed the Zumba classes with you together. I wish I could know you much earlier as a friend. Success with your rest of $\mathrm{PhD}$ ! Cengiz, take care of your stem cells and treat them well. We will keep in tough via WeChat. Greg, Nikolas, Selene, I enjoyed our greatest country tour. Good luck with your rest of $\mathrm{PhD}$ and future careers! Niko, thanks for guiding me to use fermenter and lending some chemical materials to me. I very much enjoyed our chats when sometimes walking around the coffee machine. Simone, thanks for your management in labs. All the best to you!

竞男, 每次在系里跟你闲聊吐槽（特别是系里的一些八卦）的时候都很开心, 想念下班后一起约着喝东 西，周末一起约着聚餐，出去骑车樂风的日子，祝你的博士学业顺顺利利！邹今幕，终于...你再也抽不 着我的血了, 一起待在同一个系的时间虽然很短暂，作为山东老乡，祝你实验顺利，多发文章! 
亚秋, 珊珊, 真的很开心能认识你们并成为朋友。几个在异国他乡的春节都是和你们一起渡过的, 让我 觉得很温暖。回顾那些一起吃吃喝喝的日子, 真是让人无比想念。亚秋, 羡慕你对一些事情的坚持, 比 方说跑步, 并且吉他也弹得那么好, 祝你在美国的科研和生活都顺利! 珊珊, 同是山东人, 我只能说我 酒量远远不如你啊! 希望“富婆”王老板将来不管在哪里都继续发财! 俊哥, 我们虽然是一个系的, 但是 却没什么工作上的交集。经常去你家蹭吃蹭喝, 有时候不经意的开导总是能让我豁然开朗, 最好的祝福 送给你跟珊珊! 李正文, 非常同情你博士期间几经曲折的心酸历程, 但是最后的论文和答辩都非常精彩。 在博士的最后阶段, 我们互相督促, 共同进步, 感谢! 现在你找到了满意的工作, 希望你在哪里都能躺 赢, enjoy your life! 黄盛世, 自从我搬家后, 我们就成了邻居, 谢谢你对我的帮助。楼里有时候会有奇 怪的人, 但是想到你就住在楼下, 心里会觉得安全一些。希望你学业顺利, 早日回国跟你老婆团聚!

珊, 映宜, 君君, 真的很开心跟你们相处, 只是没想到时间过得那么快, 我们刚彼此稍稍熟悉, 我就毕 业离开了, 彷佛我们一起去挪威的快乐时光就在昨天。珊, 真的很开心跟你做邻居, 你是一个内心丰富, 热爱生活的人。同时, 你对生活的热爱也激发了我对生活的探索和生活质量的要求。希望你以后的生活 更加丰富多彩! 映宜, 喜欢你大大咧咧却很真实的性格。祝你博士学业顺利, 开开心心! 君君, 很怀念 跟你和珊我们一起时不时地约下午茶, 做甜品, 聊聊天的时光, 感谢你在我回国前的最后的几天给我提 供住所, 祝你博士学业顺利, 早日回京!

还有我在马城认识的其他的小伙伴, 郭乐（在马城第一年的室友）, 萋英（马城 PhD 联络员和马城博士 界的学术标杆），孙傲敏（谢谢你在我搬家时收留了我几天），冬梅, 关关（一起去挪威），黄䂀（怀 念一起去德国, 卢森堡旅游的日子），湘兰妹子（马城永远欢迎你, 最然我不在马城了, 欢迎来上海）, 秋婷（马城吃吃喝喝组），毕（湘崎）（遗憾 fencing 没能上几节课, 我们都是被疫情耽误的 fencers）, 情怡（可爱的小学妹，95 后，希望你感情顺利，学业有成） …...

最后，我要感谢我的父母和家人，你们总是给予我足够的自由让我自己来做决定，选择自己想要的人生。 你们给予我极大的支持和鼓励, 让我在生活和学习中总是有一种绝不气馁和妥协的信念, 我也很开心能 够成为你们的骄傲。爸爸妈妈, 每次回国看到你们一年年变老, 也让我责任感随之增强, 渐渐成长。还 有我的弟弟妹妹们, 虽然我们年龄差很多, 但是也正是年龄的差距让我有机会见证你们从出生到成人, 到长得比我高比我壮, 以前是我照顾你们, 现在我每次回国, 都是你们来照顾我, 我感受到了更多来自 家庭的爱! 希望我们一家人健健康康, 平安幸福!

最后的最后, 感谢我的男朋友薛云鹏。亲爱的, 我们从谈恋爱到现在已经将近十年, 我们是最熟悉彼此 的人。真的非常感谢你一直以来的陪伴和支持, 谢谢你倾听我的牢骚, 谢谢你对我的开导, 谢谢你对我 的包容! 